\title{
Environmental Regulatory Update Table
}

\author{
January/February 1992
}


This report has been reproduced directly from the best available copy.

Avallable to DOE and DOE contractors from the Office of Scientfic and Techntcal Information, P.O. Box 62, Oak Ridgo, TN 37831; pricos available from (615) 57E-B401, FTS 628-8401.

Avallable to the public from the National Technical Information Service, U.S. Department of Commerce, 5285 Port Royal Rd., Springfield, VA 22161.

This report was prepared as an account of work sponsored by an agency of the United States Government. Nelther the United States Government nor an; agency thereot, nor any of their employees, makes any warranty, express or implied, or assumes any legal liability or responsibilty for the accuracy, completeness, or usefulness of any information, apparatus, product, or proceses dieclosed, or represente that its use would not infringe privately owned rights. Reference herein to any spectilic commercial product, process, or service by trade narne, trademark, manufacturer, or otherwise, does not necsesarity constitute or imply Its endorsement, recommendation, or favoring by the Untied States Government or any agency thereot. The views and opinions of auttrors expressed herein do not necessarily state or reflect those of the United States Government or any agency thereot. 


\section{ENVIRONMENTAL SCIENCES DIVISION}

ENVIRONMENTAL REGULATORY UPDATE TABLE

JANUARY/FEBRUARY 1992

L. M. Houlberg*

G. T. Hawkins"

M. S. Salk.

"Health \& Safety Research Division

Environmental Sciences Division

Publication No. 3643

Date Published - March 1992

Prepared for the

U.S. Department of Energy

Office of Environmental Guidance

Lois Thompson

Technical Project Manager

(B/A No. HA 011500 0; EHHA002)

Prepared by the

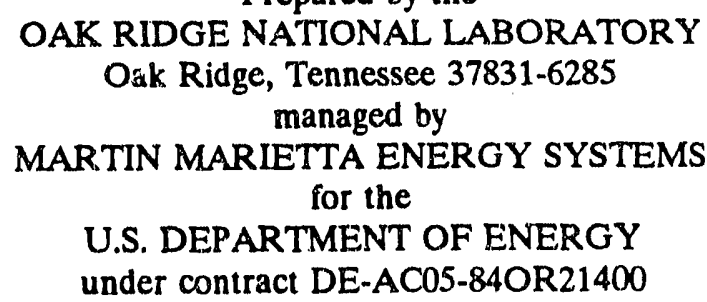

MASTER 
Date

\section{CHANGES IN REGULATORY UPDATE TABLE DISTRIBUTION}

Please modify the distribution for the Environmental Regulatory Update Table by (check one):

$\begin{array}{ll}\text { - } & \text { Adding: } \\ \text { _ } \quad \text { Deleting: }\end{array}$

- Correcting the address as indicated:

- Removing and adding:

Name:

Address:

Phone: (Commercial)

(FTS)

Please send this form to:

\section{Linda Houlberg}

Oak Ridge National Laboratory

P. O. Box 2008

Building 20)1, Mail Stop 6050

Oak Ridge, TN 37831-6050

Or call her at FTS: $624-7763$, Commercial: (615) 574-7763 


\section{Environmental Regulatory Update}

This report contains a bi-monthly update of environmental regulatory activity that is of interest to the Department of Energy. It is provided to DOE operations and contractor staff to assist and support environmental management programs by tracking regulatory developments. Any proposed regulation that raises significant issues for any DOE operation should be reported to the Office of Environmental Guidance (EH-23) as soon as possible so that the Department can make its concerns known to the appropriate regulatory agency. Items of particular interest to EH-23 are indicated by a shading of the RU\#.

Any questions or comments about any aspects of the Update Table should be directed to Lois Thompson, Air, Water, and Radiation Protection Division, Office of Environmental Guidance, U.S. Department of Energy (EH-23), (FTS) 896-9581, (Commercial) 202-5869581. 


\begin{abstract}
The Environmental Regulatory Update Table provides information on regulatory initiatives of interest to DOE operations and contractor staff with environmental management responsibilities. The table is updated bi-monthly with information from the Federal Register and other sources, including direct contact with regulatory agencies. Each table entry provides a chronological record of the rulemaking process for that initiative with an abstract and a projection of further action.
\end{abstract}




\section{U.S. DEPARTMENT OF ENERGY}

ASSISTANT SECRETARY FOR ENVIRONMENT, SAFETY AND HEALTH Office of Environmental Guidance

ENVIRONMENTAL REGULATORY UPDATE

JANUARY/FEBRUARY 1992 


\section{REGULATORY UPDATE TABLE - JANUARY/FEBRUARY 1992 UPDATE}

Table of Contents

$\begin{array}{lll}2.20 & \text { Page }\end{array}$

Regulatory Highlights $\quad$ xiii

Regulatory Update Table $\quad 1$

Resource Conservation and Recovery Act (RCRA) (RU \# 840-...) $\ldots \ldots \ldots \ldots \ldots 1$

Comprehensive Environmental Response, Compensation, and

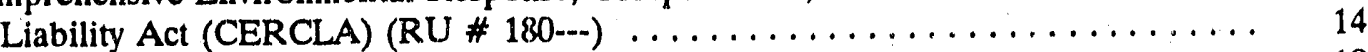

Superfund Amendments and Reauthorization Act (SARA) (RU \# 190-... . . . . . . . 19

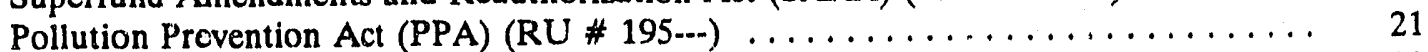

Safe Drinking Water Act (SDWA) (RU \# 880 -.) $\ldots \ldots \ldots \ldots \ldots \ldots \ldots$. . . . . . . . . 22

Clean Water Act (CWA) (RU \# 140 $\ldots$. $\ldots \ldots \ldots \ldots \ldots \ldots \ldots \ldots \ldots \ldots \ldots$

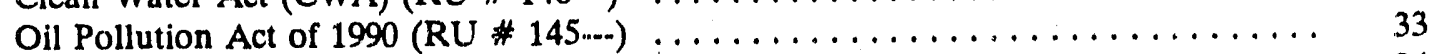

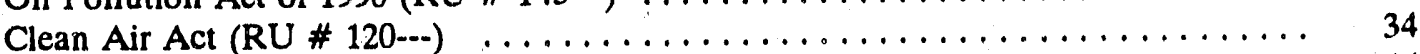

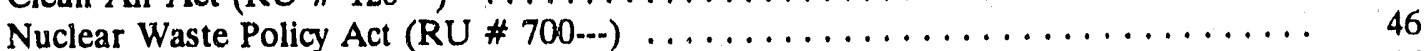

Low-Level Radioactive Waste (LLW) Policy Amendments Act (RU \# 740---) . . . . . 47

Atomic Energy Act (RU \# 100-..) . . . . . . . . . . . . . . . . . . . . . . . . . 49

Uranium Mill Tailings Radiation Control Act (RU \# 930 ...) . . . . . . . . . . . . 59

Toxic Substances Control Act (TSCA) (RU \# 920 m) . . . . . . . . . . . . . . . . 59

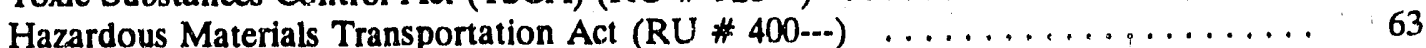

Occupational Safety and Health Act (OSHA) (RU \# 780 - . . . . . . . . . . . . . 69

Endangered Species Act (RU \#200 $\ldots \ldots \ldots \ldots \ldots \ldots \ldots \ldots \ldots$

Marine Protection, Research, and Sanctuaries Act (RU \# 460-..) . . . . . . . . . . . 79

National Environmental Policy Act (RU \# 480 ...) . . . . . . . . . . . . . . . . . 79

Archeological Resources Protection Act (RU \# 090-..) . . . . . . . . . . . . . . . 80

Federal Insecticide, Fungicide, and Rodenticide

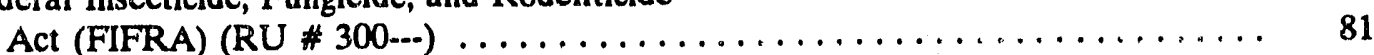

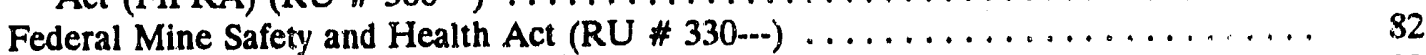

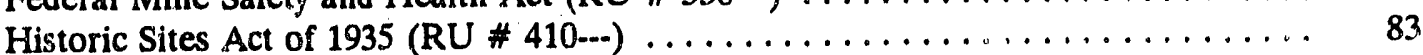

Biotechnology (RU \# 110-.) $\ldots \ldots \ldots \ldots \ldots \ldots \ldots \ldots \ldots \ldots \ldots \ldots$

Pacific Northwest Electric Power \& Conservation (RU \# 810 ...) . . . . . . . . . . 83

Coastal Zone Management Act $(\mathrm{RU} \# 160-\ldots) \ldots \ldots \ldots \ldots \ldots \ldots \ldots$

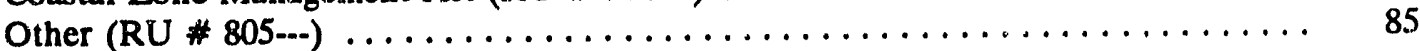

IRIS Table Update $\quad 87$

Index to RU Numbers by CFR Citation 105

"RU numbers are identifiers that are assigned to each regulatory initiative fir rrarking purposes. The first three digits are coded to the statute, and the last three digits are speciffi to individual initiatives. The update table is first organized by statutes as shown abuse and then by RU numbers arranged sequentially. An index is provided at the end of the table to selare CFr citations to RU numbers. 
Regulatory Highlights - January/February, 1992

\section{Liners and Leak Detection Systems for Hazardous Waste Land Disposal Units - Final Rule}

Under the Resource Conservation and Recovery Act, EPA issues a final rule (NPRMs: 3-28-86 and 5-29-87) establishing new and amended standards in 40 CFR parts 260, 264, 265,270 , and 271 for affected hazardous waste land disposal units, regardless of their permit status. The rule sets standards intended to minimize the potential for migration of hazardous constituents from hazardous waste land disposal units to underlying ground water. The amended standards concern liner and leachate collection and removal system requirements for new and replacement surface impoundments, landfills, and waste piles and for lateral expansions of these units. The new standards require installation of an approved leak detection system at such units. For purposes of the rule, a "new unit" is defined as a unit for which construction begins after 1-29-92, the promulgation date of the rule. A replacement unit is defined as a unit "(1) from which all or substantially all of the waste is removed, and (2) that is subsequently reused to treat, store, or dispose of hazardous waste." The revised standards require that affected units have a double liner with a top liner designed to prevent the migration of hazardous constituents into the liner during the unit's active life and post-closure period and a composite bottom liner consisting of a geomembrane on top of at least three feet of compacted soil material having a hydraulic conductivity no greater than $1 \times 10-7 \mathrm{~cm} / \mathrm{sec}$. The rule also requires installation and operation of leak detection systems at regulated units capable of detecting a leak through the top liner at the "earliest practicable time". EPA interprets that as the time lapse from when a liquid passes through a breach in the top liner to when a technology-based leak detection system can detect the liquid, assuming saturated, steadystate flow. Such a leak detection system (designated by EPA as the leachate collection and removal system drainage layer located immediately above the bottom composite liner) will have to meet several design criteria set forth in the rule. In addition to the core requirements, the Agency also promulgated several complementary standards in the rule to enhance the overall effectiveness of liners and leak detection systems at applicable units. One of these accompanying standards is the development of a response action plan to address the detection of a leakage rate greater than a pre-determined site-specific leakage rate, the action leakage rave. Further protective measures incorporated in the regulations include requirements for monitoring and inspection. The rule will become effective on July $29,1992$.

(1-29-92, 57 FR 3462, RU\# 840-109 \& 840-138)

\section{Land Disposal Restrictions for Newly Listed Wastes and Contaminated Debris - Proposed Rule}

As required by section $3004(\mathrm{~g})(4)$ of the Resource Conservation and Recovery Act (RCRA), EPA proposes (ANPRM: 5-30-91) land disposal prohibitions and corresponding treatment standards for certain "newly listed and identified hazardous wastes" (i.e., wastes 
identified or listed after the 1984 Hazardous and Solid Waste Amendments to RCRA). The 20 newly listed wastes covered in the notice are certain F- and K-listed wastes and three organic $U$ wastes. (EPA designates hazardous wastes using an alphanumeric code that begins with a capital letter followed by three numbers.) The proposal describes the treatment and/or recycling technologies identified as the Best Demonstrated Available Technologies (BDAT) for meeting the treatment standards proposed for the newly listed wastes. The proposed standards are based on performance data from treating other similar hazardous wastes. The Agency is also proposing revisions to (1) land disposal restriction (LDR) treatment standards applicable to debris contaminated with certain listed hazardous wastes or that exhibit a hazardous waste characteristic, and (2) other existing LDR treatment standards and requirements. EPA is proposing to require the treatment of contaminated debris using specified technologies based on the type of debris and the contaminants present. All of the treatment technologies identified as BDAT for contaminated debris wastes fall within the general categories of extraction, destruction, or immobilization. For debris contaminated with mixed waste, the Agency is proposing to require compliance with treatment standards for contaminated debris (rather than standards for the contaminating hazardous waste) in addition to any regulation of that material under the Atomic Energy Act. The proposed revisions to current LDR regulations include: (1) establishment of treatment standards for the F001-F005 spent solvent wastes based on total concentration of organic constituents in the waste rather than on the Toxicity Characteristic Leaching Procedure, (2) modification of the concentration-based treatment standards for wastewater forms of $24 \mathrm{~K}$-and U-listed wastes, (3) amended notification and certification requirements pertaining to the tracking of characteristic hazardous wastes, and (4) a revised definition of "pile" to exclude containment buildings. EPA proposes a new regulatory definition of containment building which would allow the storage/treatment of certain prohibited wastes in these structures provided certain design and operating requirements are complied with. Comments on this proposed rule were to be submitted to the Agency by February 24, 1992.

(1-9-92, 57 FR 958, RU\# 840-146)

\section{Hazardous Waste Management System: Identification and Listing of Hazardous Waste - Final Rule}

On May 19, 1980, EPA promulgated a number of criteria (40 CFR 261.11) for listing wastes as hazardous as part of its regulations implementing section 3001 of the Resource Conservation and Recovery Act (RCRA). On July 19, 1991, EPA proposed to change the language to reflect the agency's current intent and consistent interpretation of that regulation. Originally the provision stated that EPA would list a waste as toxic if the waste contained any toxic constituent listed in appendix VIII of 40 CFR 261 unless, after considering a series of enumerated factors, the Administrator determined that the waste would not pose a substantial hazard to human health and environment even if managed improperly. (Appendix VIII contains a list of substances shown in scientific studies to be toxic, carcinogenic, mutagenic, or teratogenic at certain concentrations.) Some individuals had maintained that this meant that any waste containing an appendix VIII constituent was presumed to be hazardous. However, EPA in actual practice always has evaluated 
relevant waste factors in its specific listing actions and then made a determination as to whether wastes containing an appendix VIII constituent are capable of causing substantial harm if mismanaged. Therefore, section 261.11(a)(3) has been revised to read as follows: "(Solid waste) contains any of the toxic constituents listed in appendix VIII and, after considering the following factors, the Administrator concludes that the waste is capable of posing a substantial present or potential hazard to human health or the environment when improperly treated, stored, transported or disposed of, or otherwise managed." The new wording continues EPA's original interpretation of the rule. The rule was effective January 13, 1992.

(1-2-92, 57 FR 12, RU\# 840-288) 


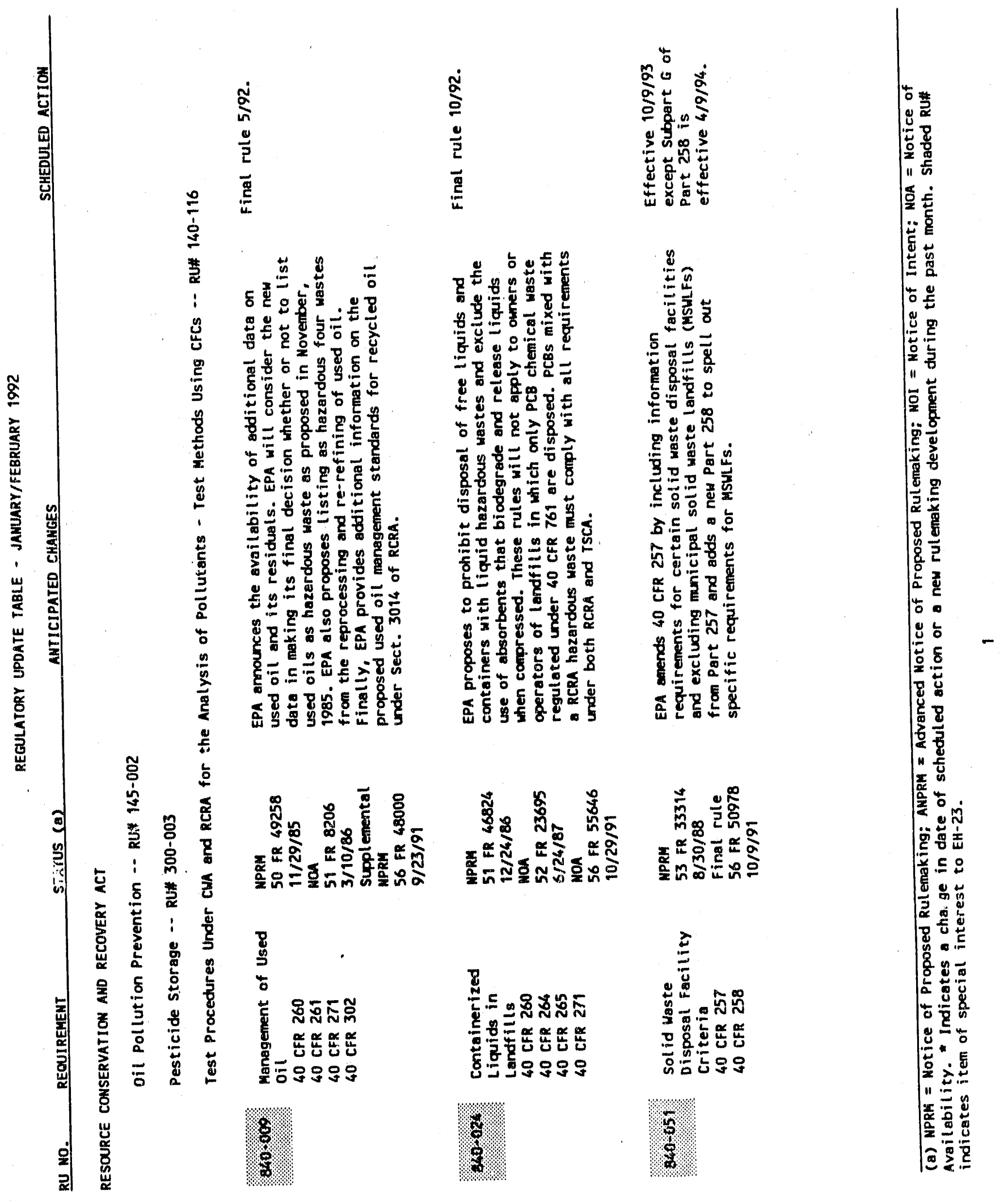




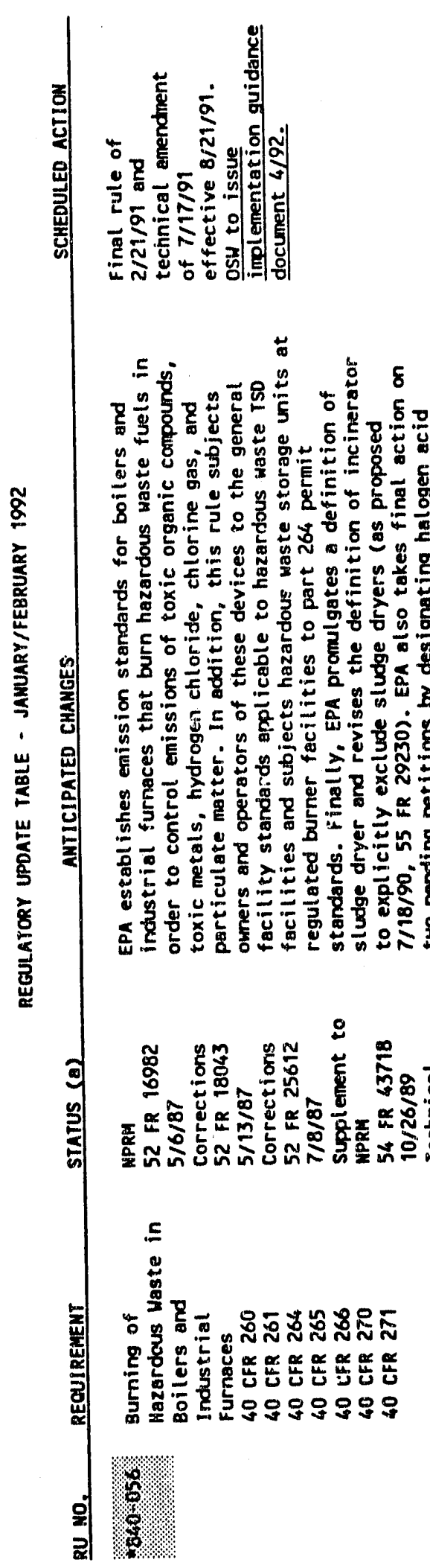

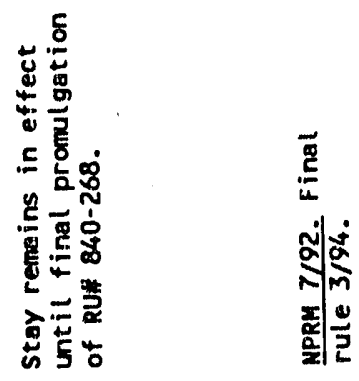

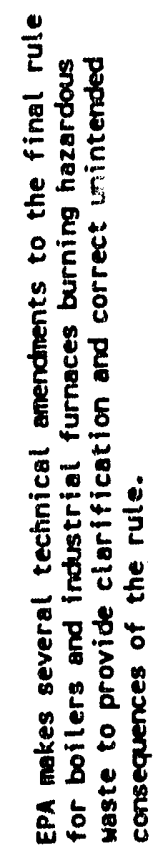

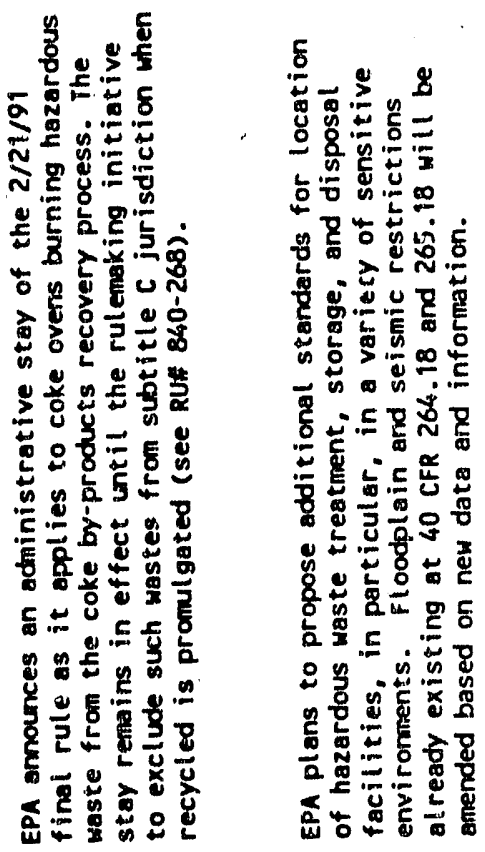



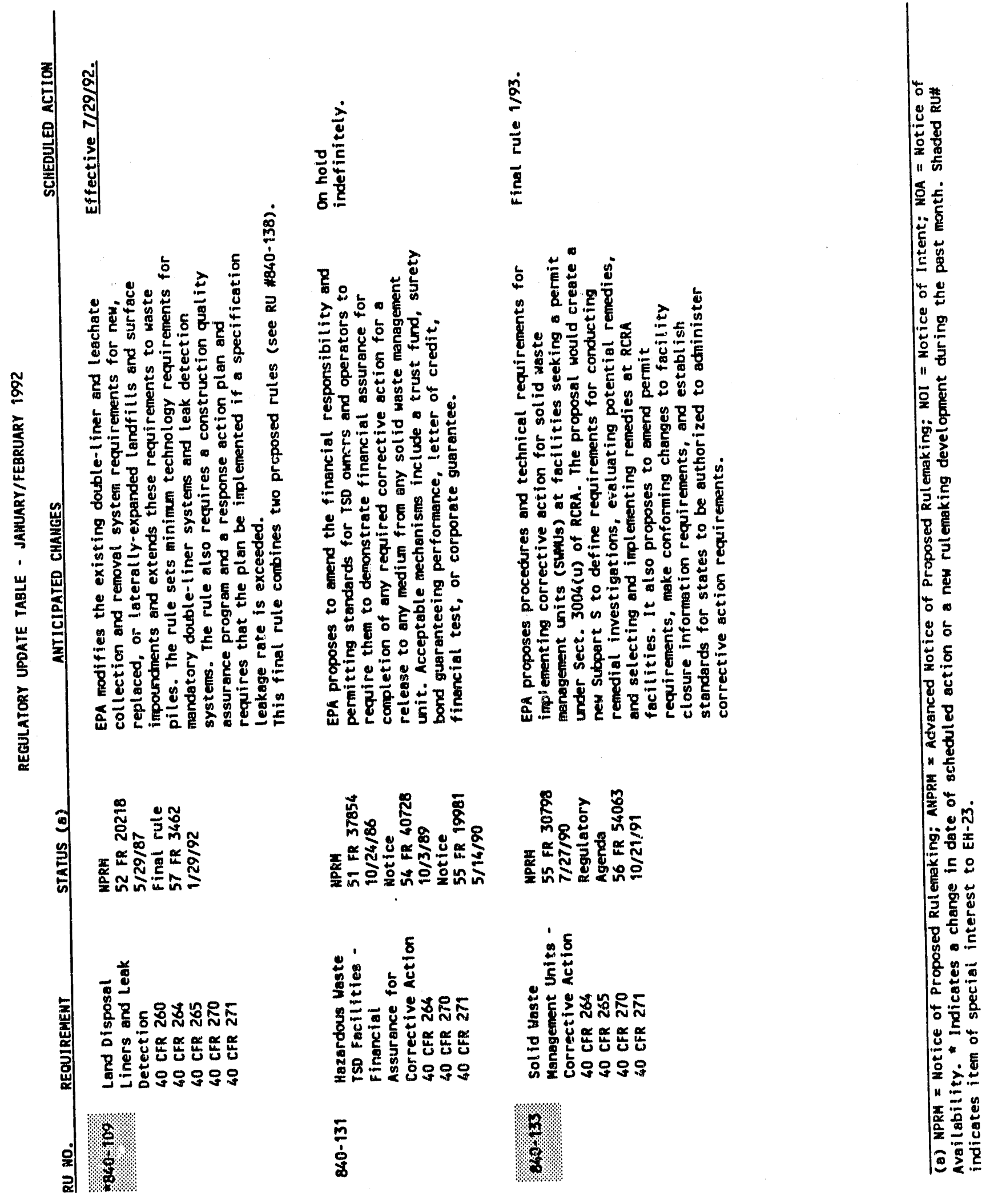


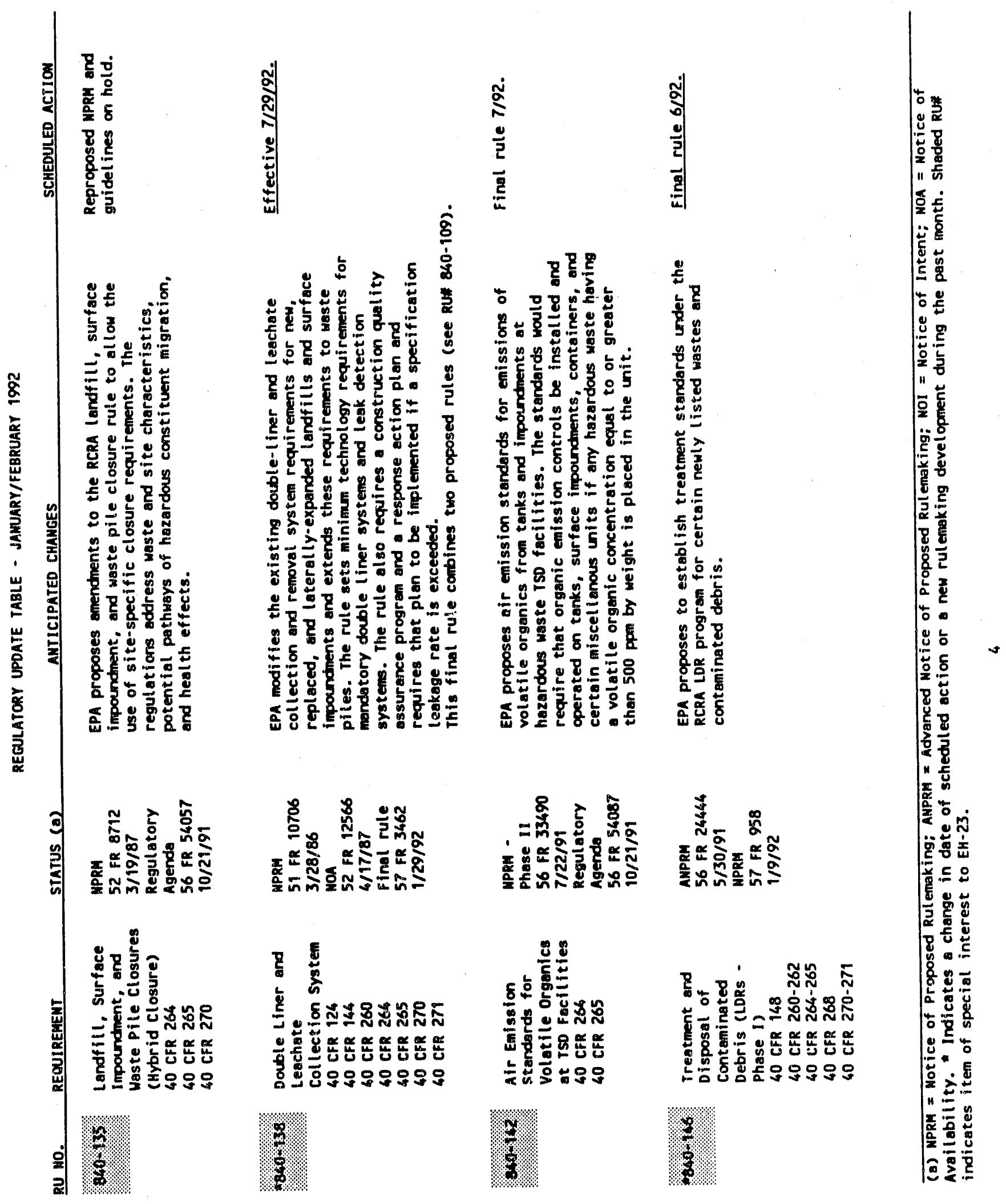




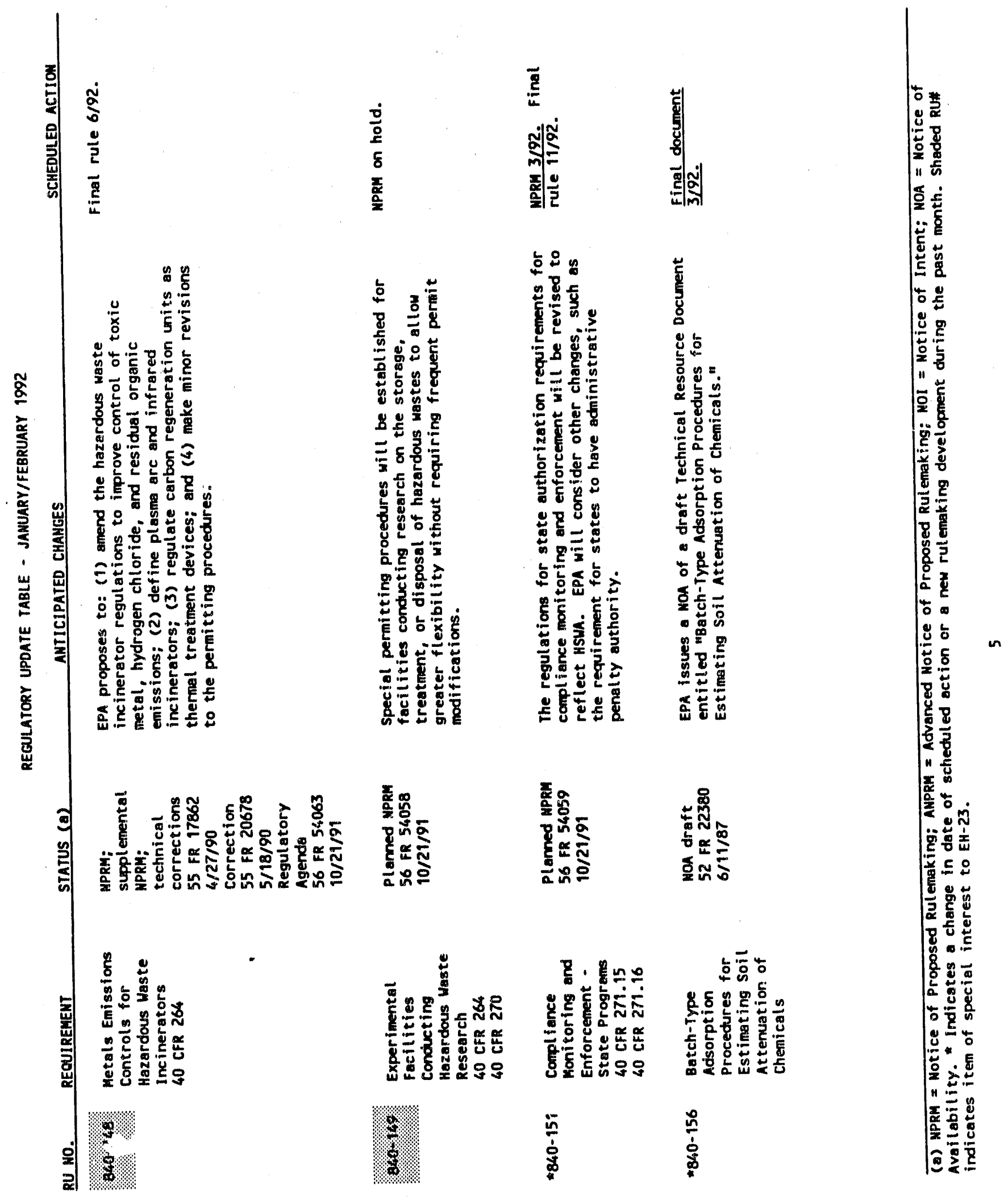




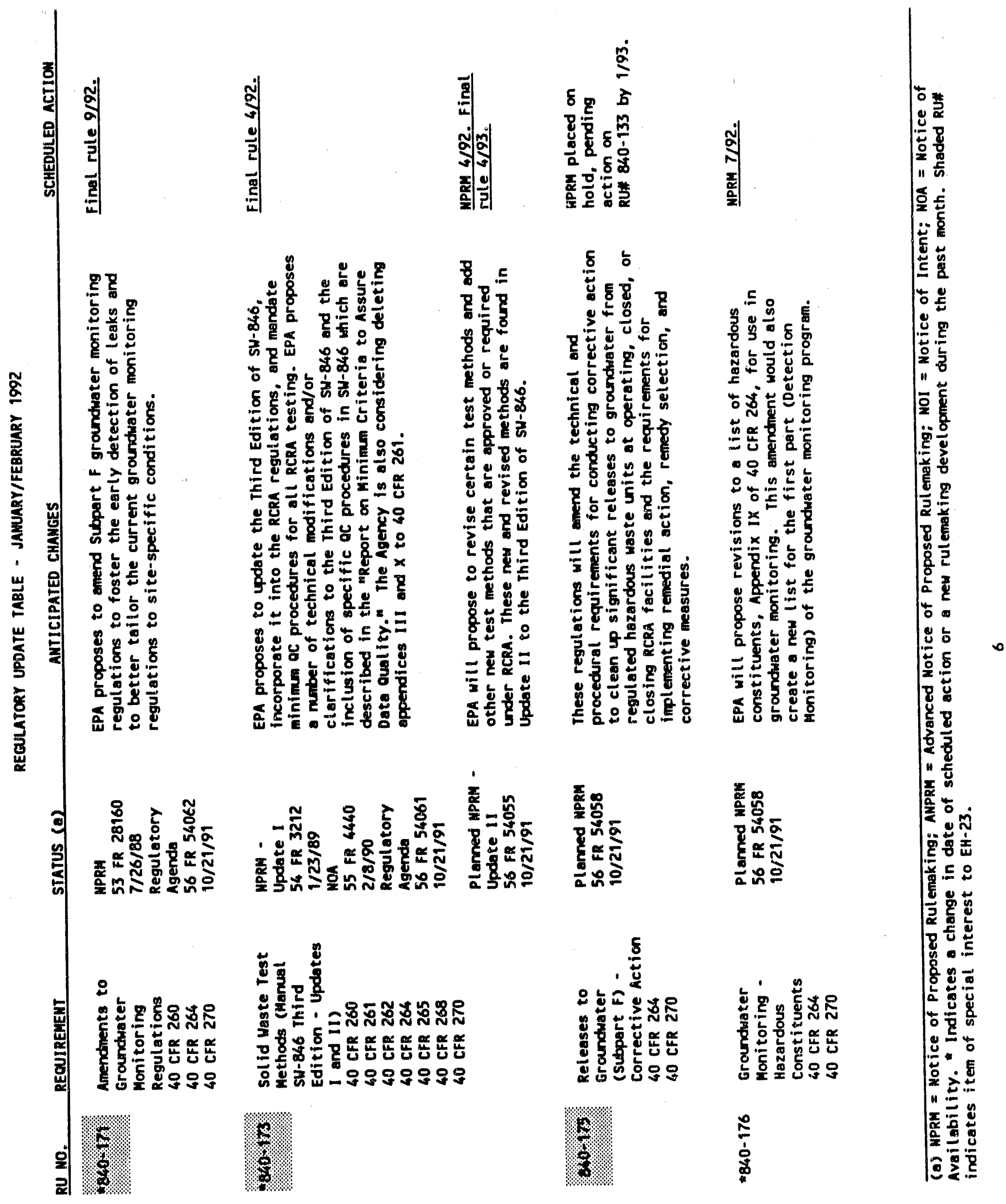




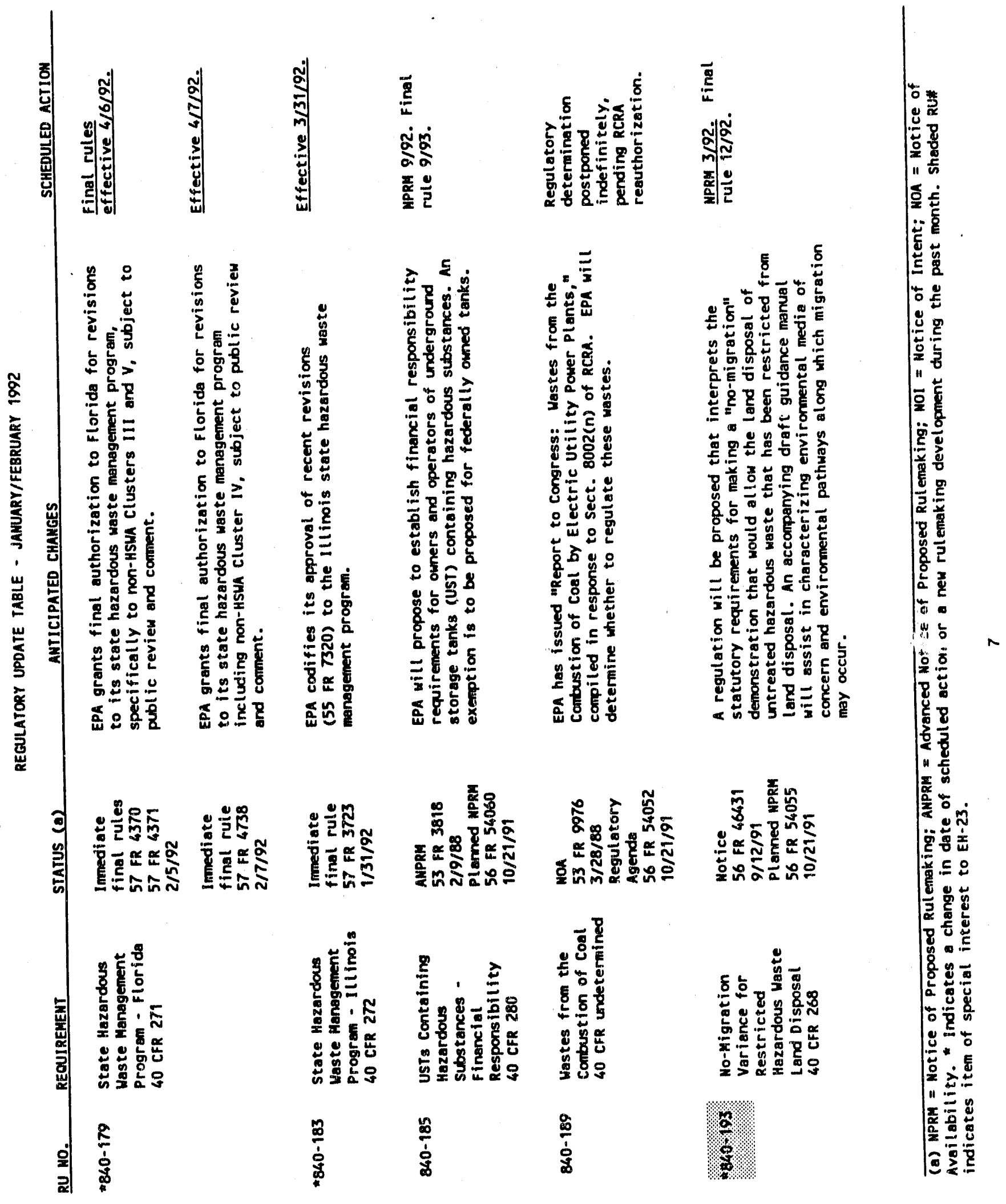




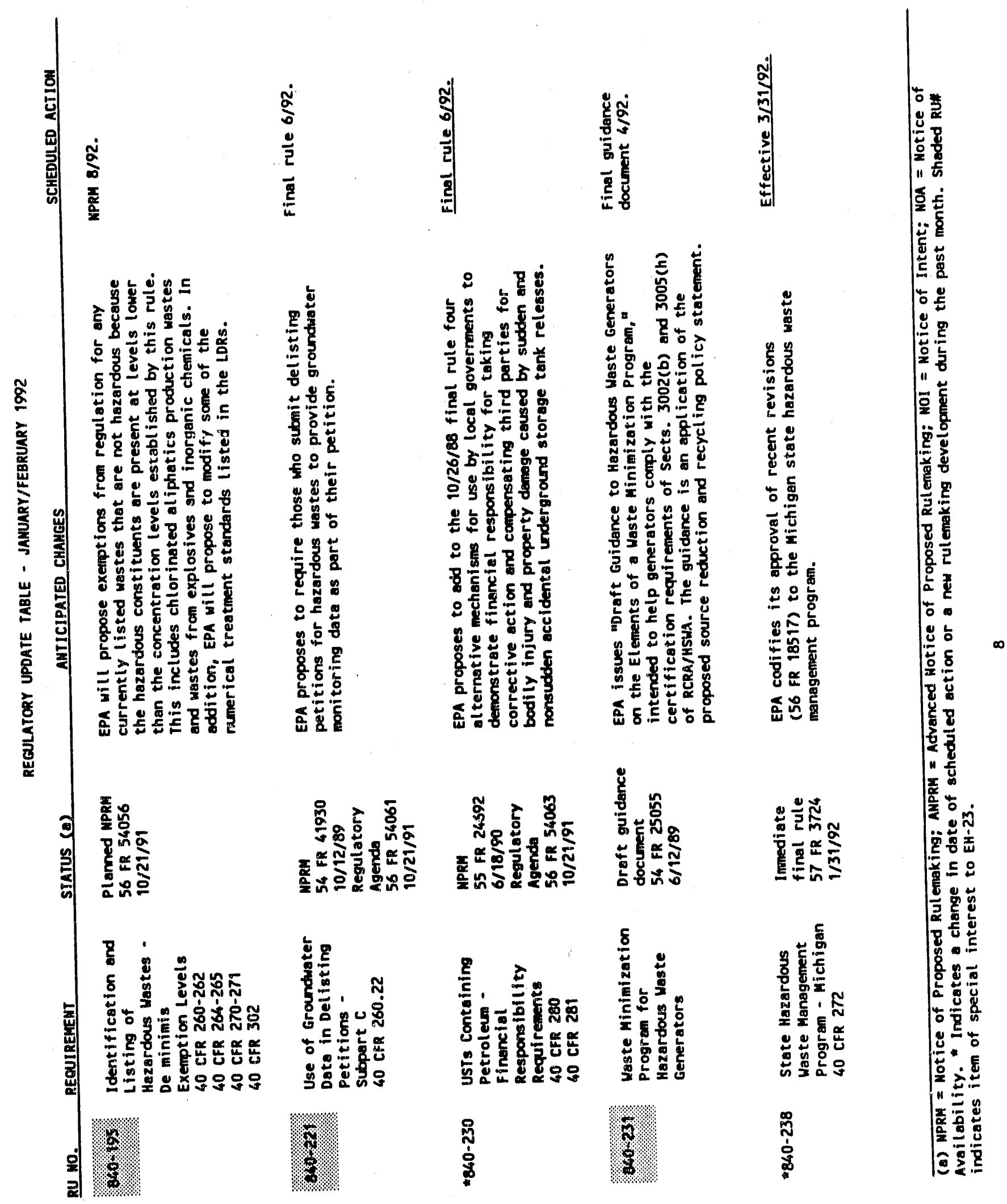




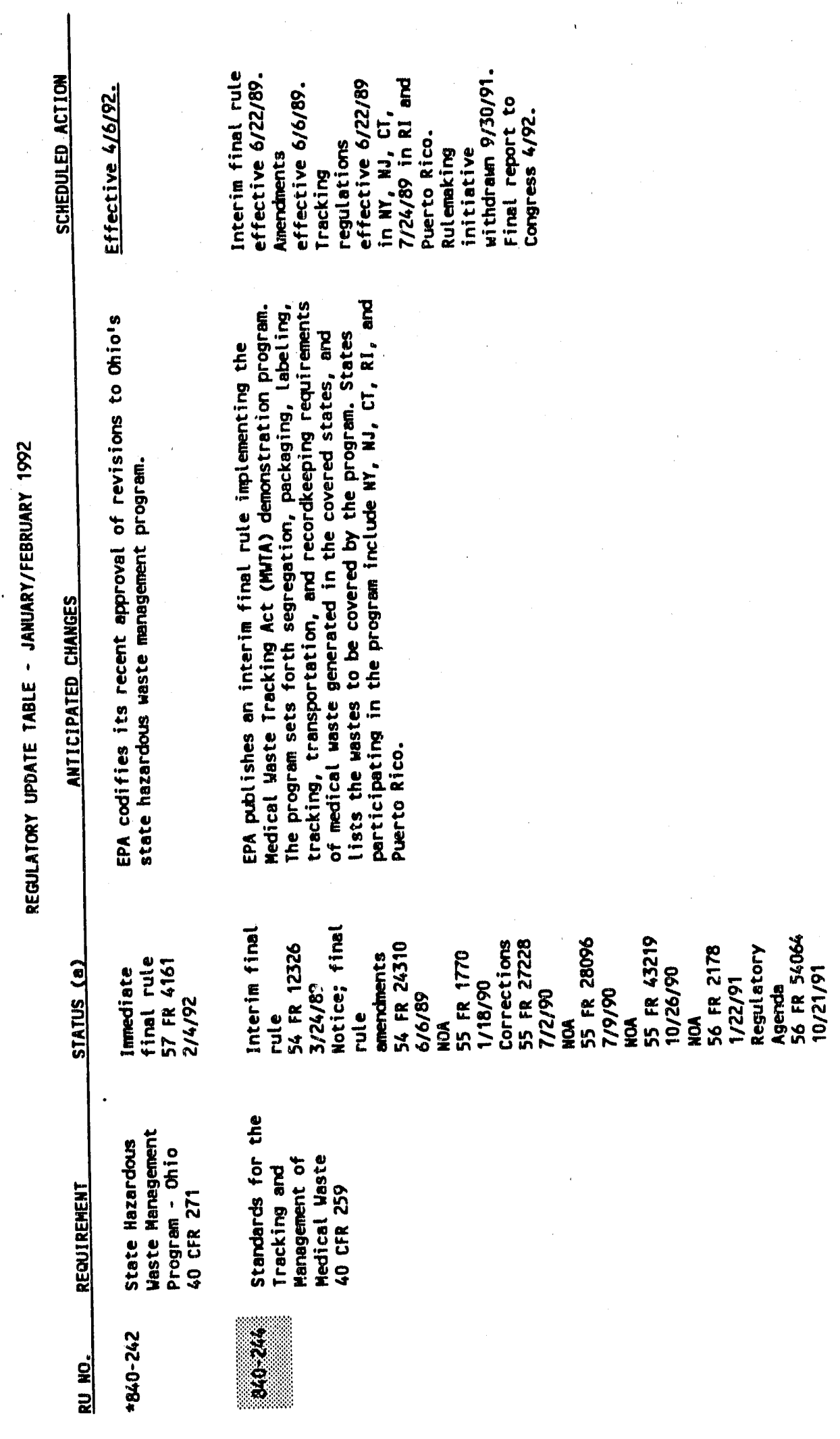



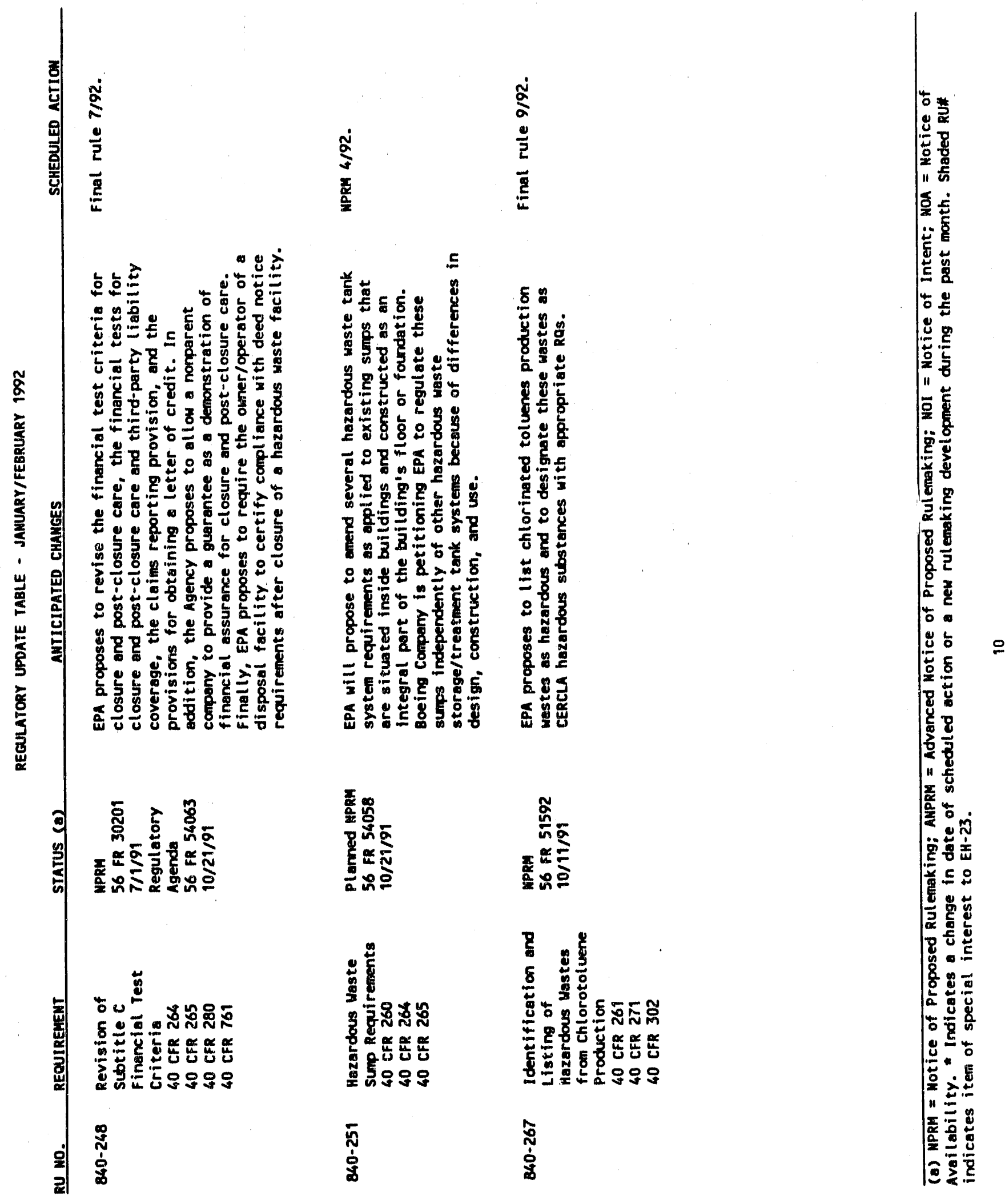


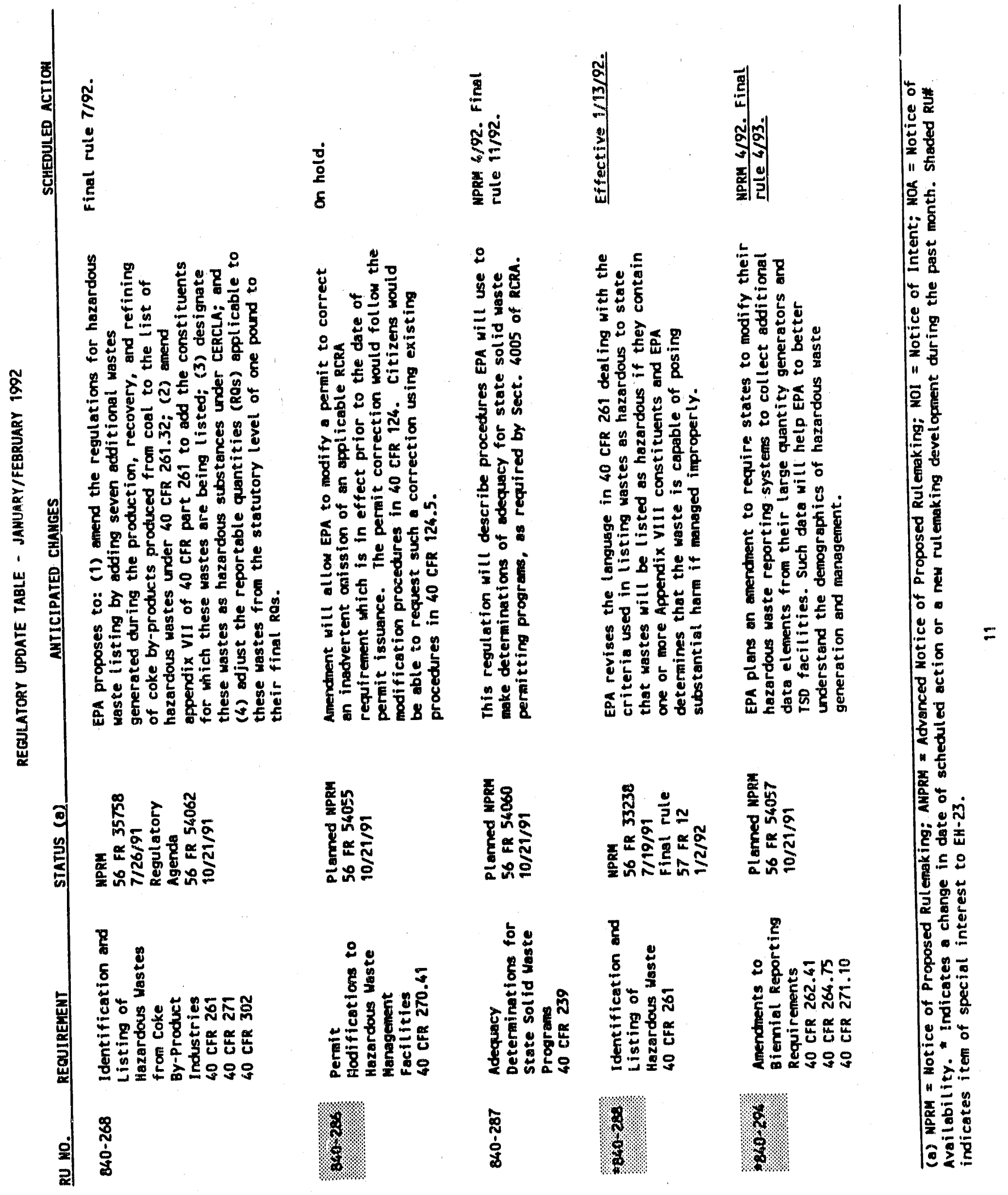



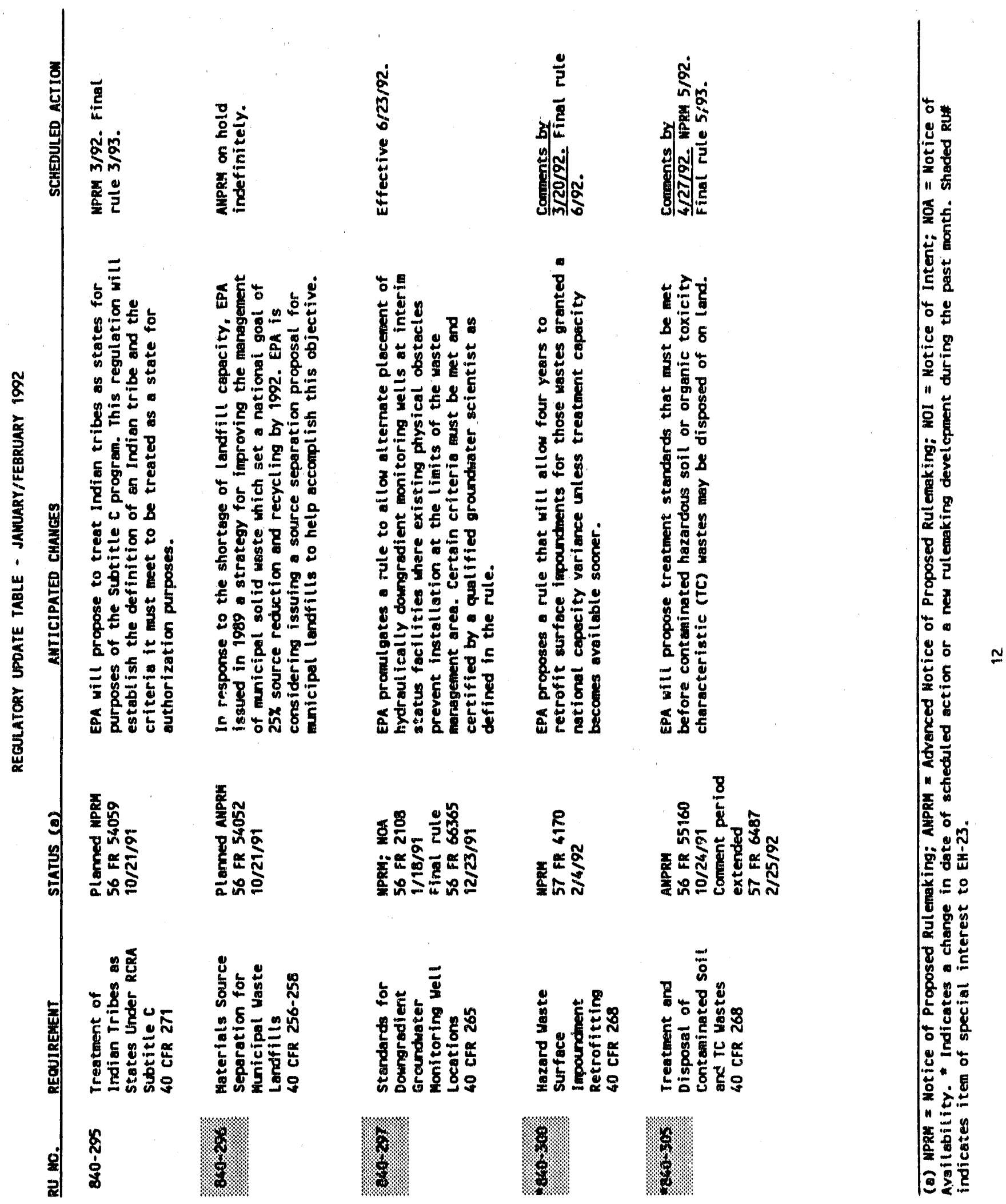

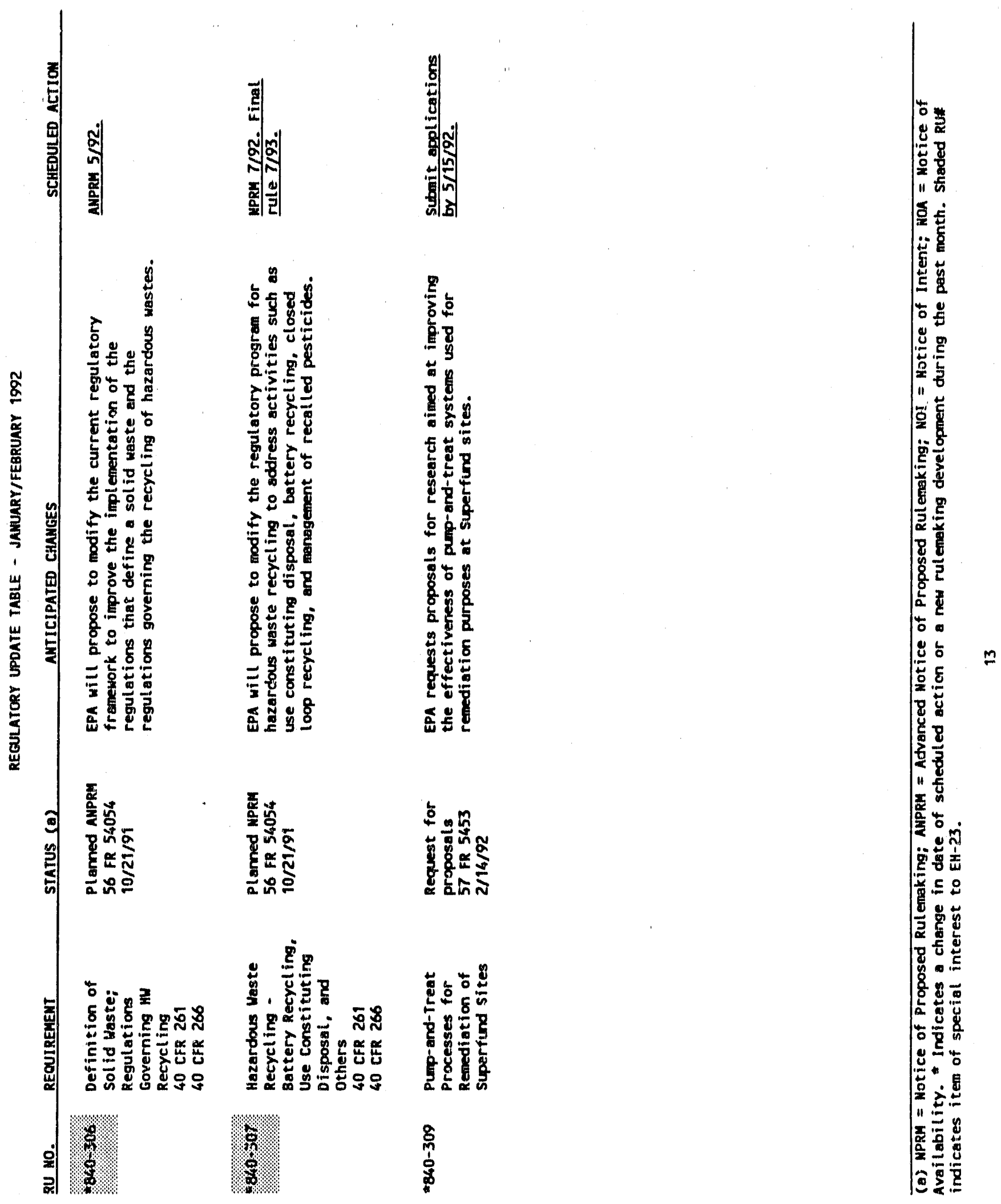

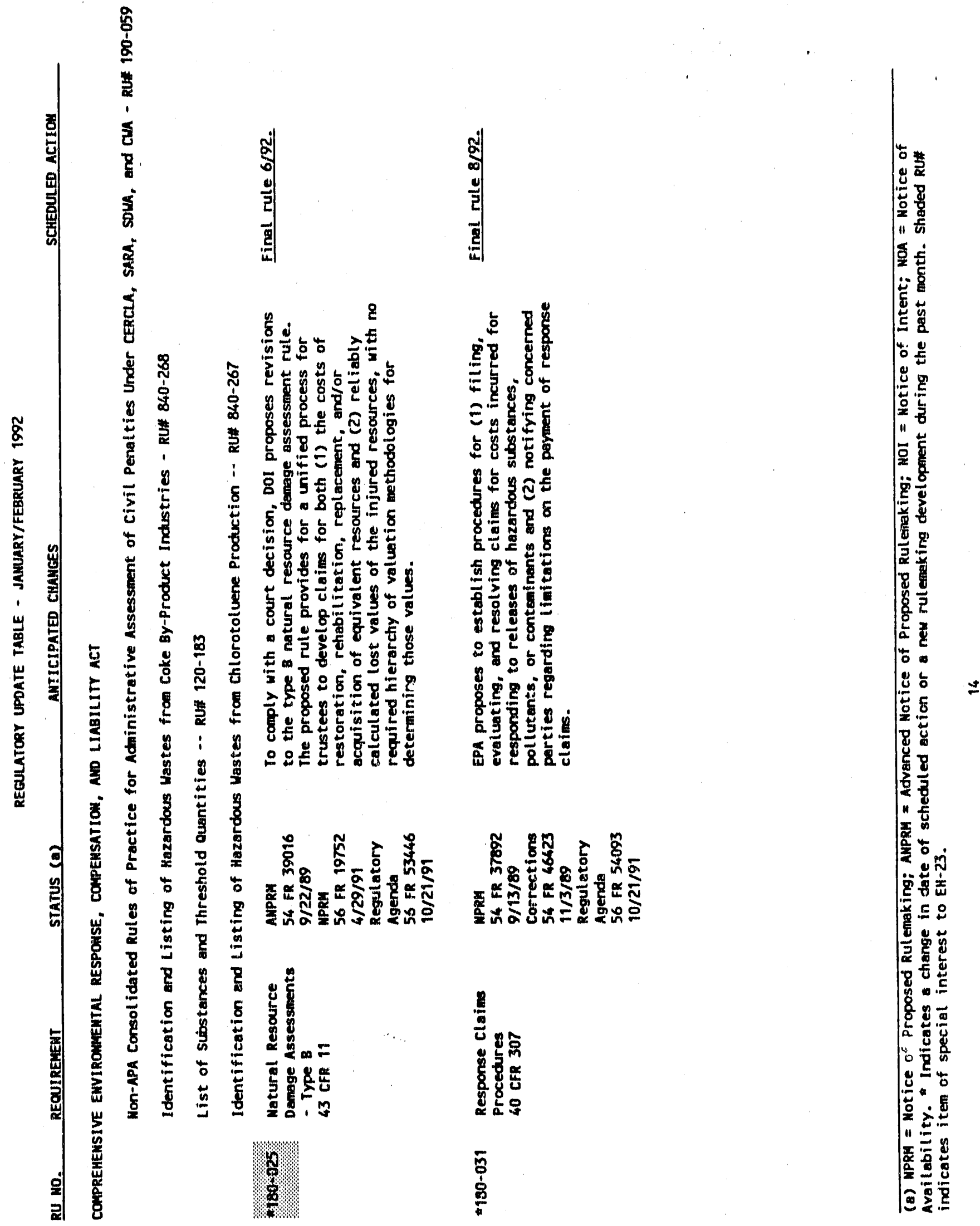


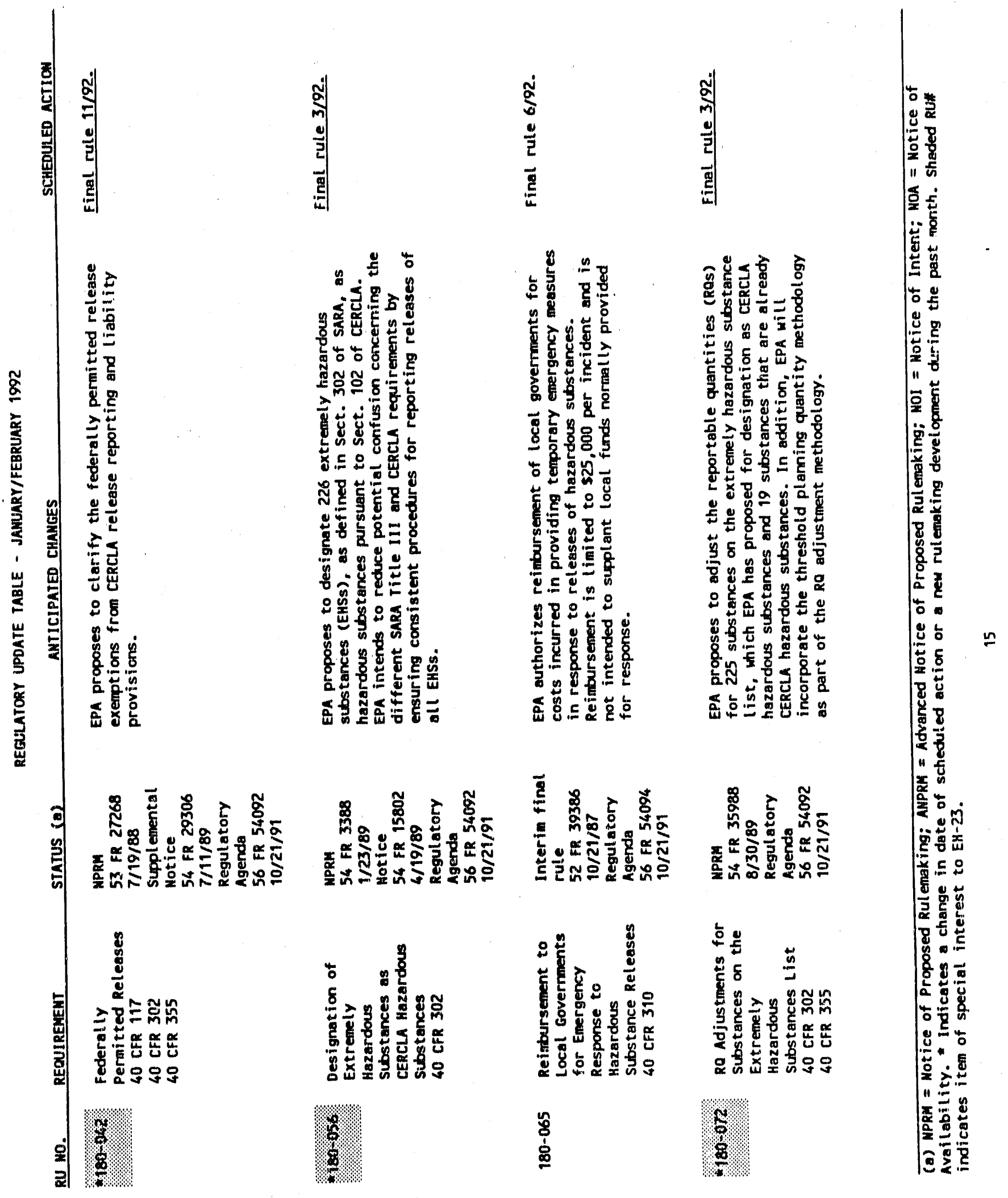




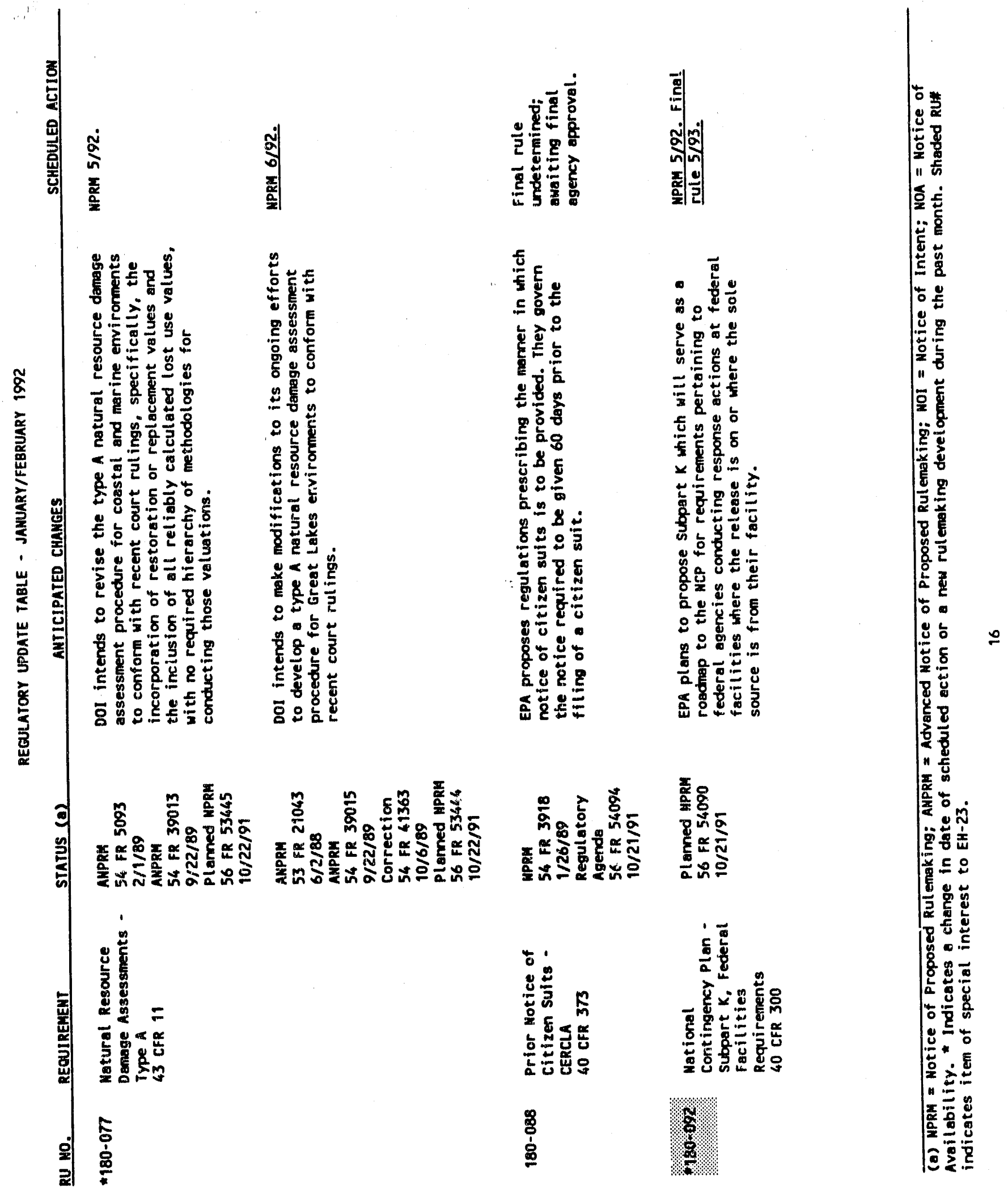




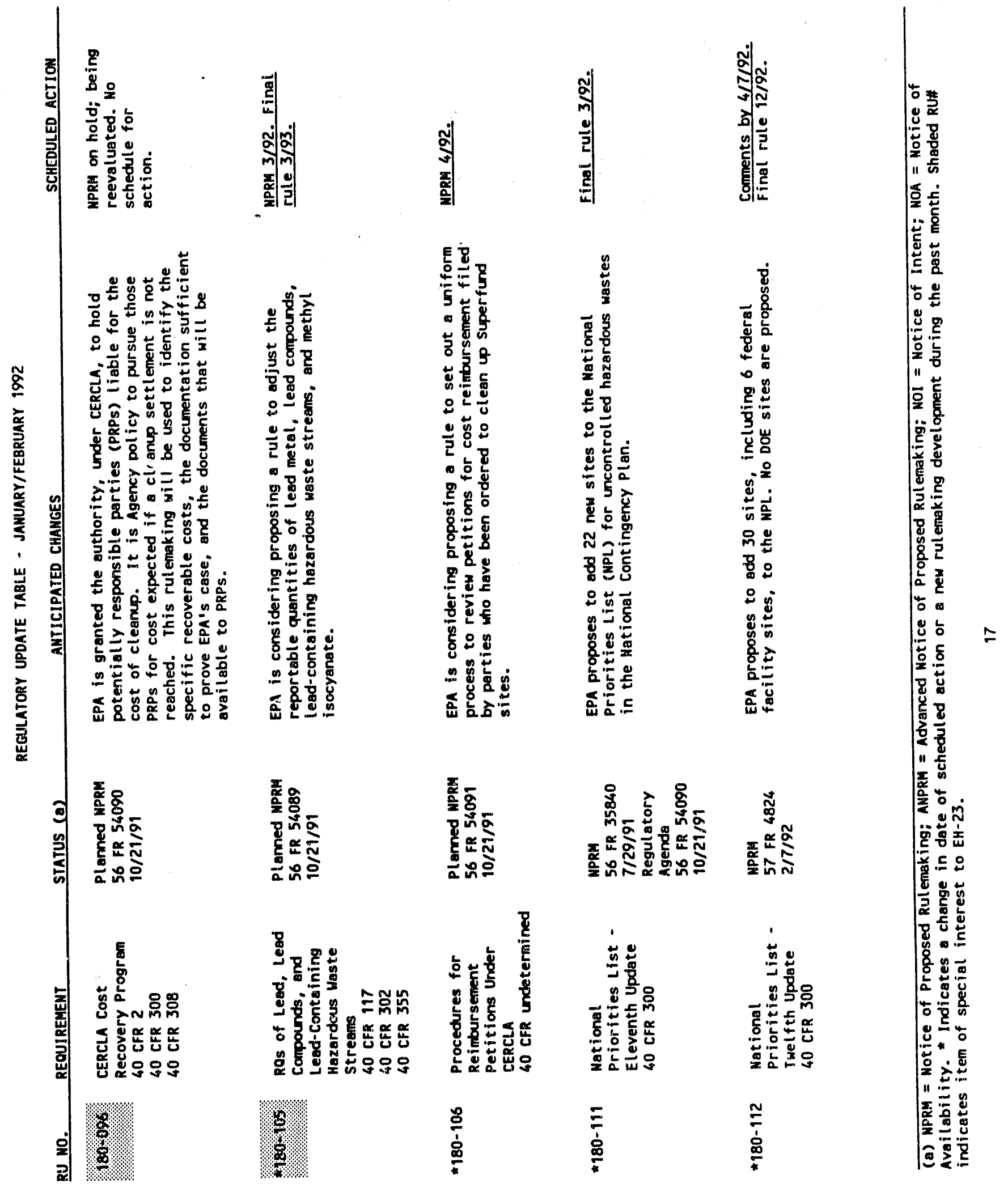



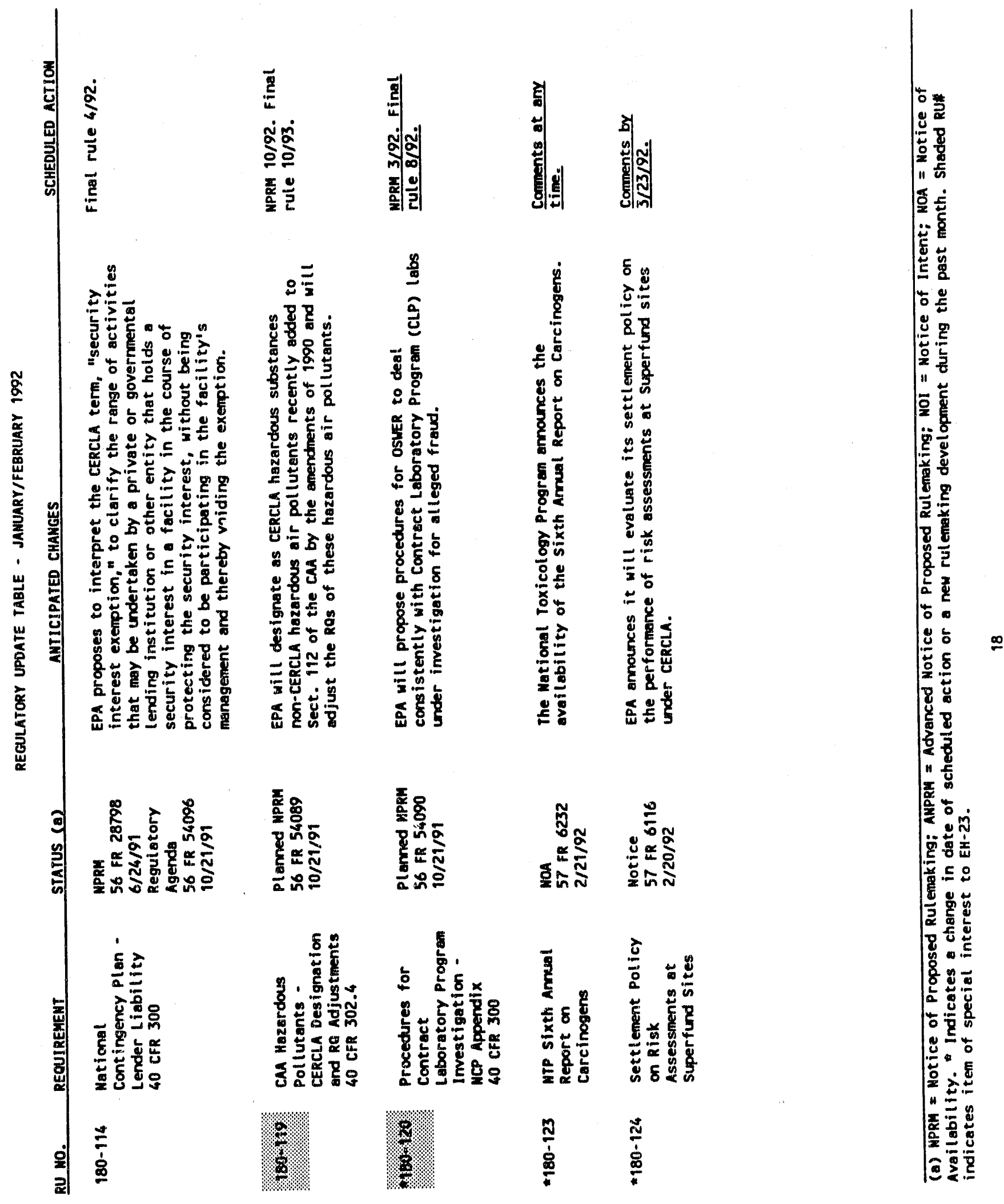


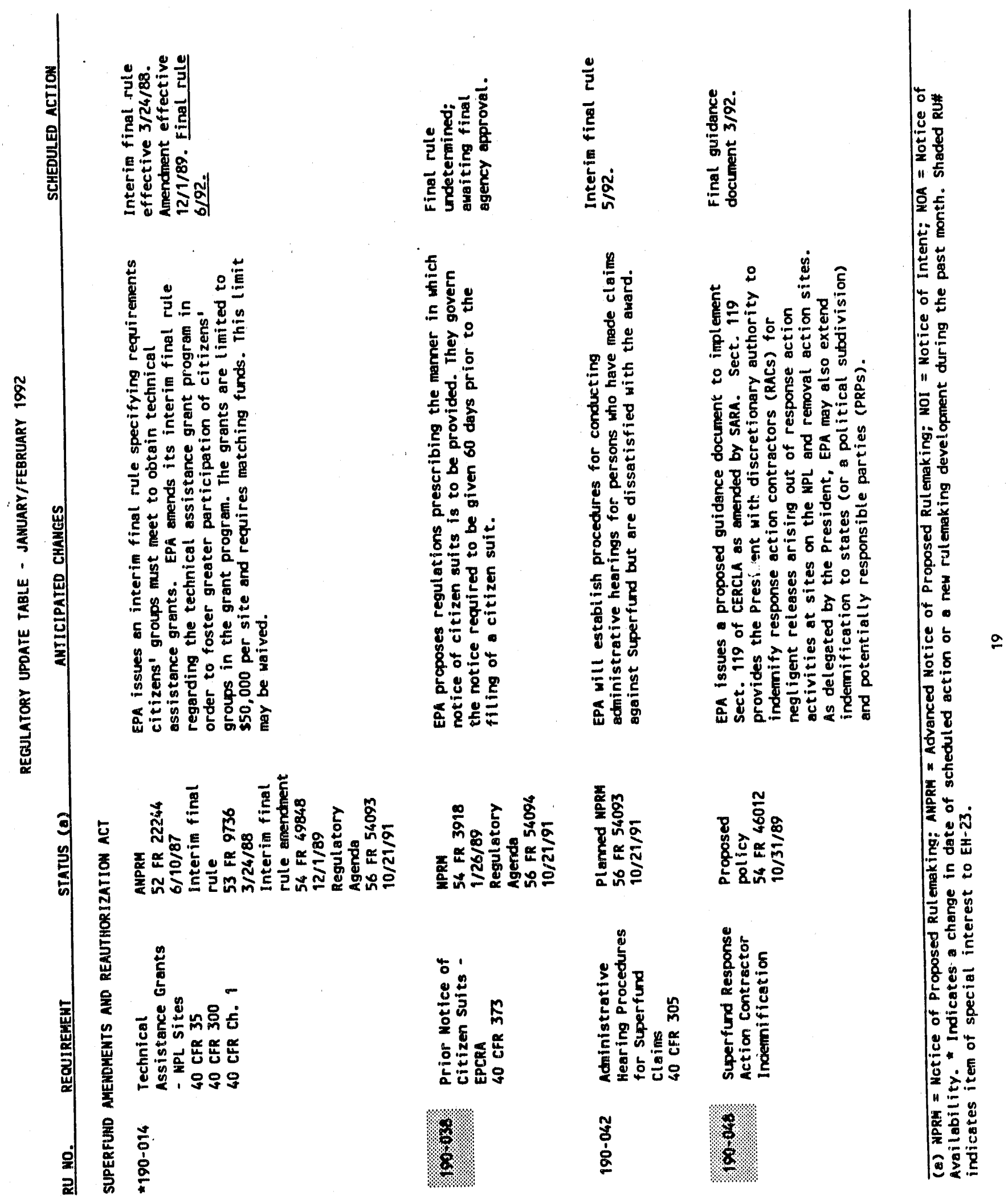




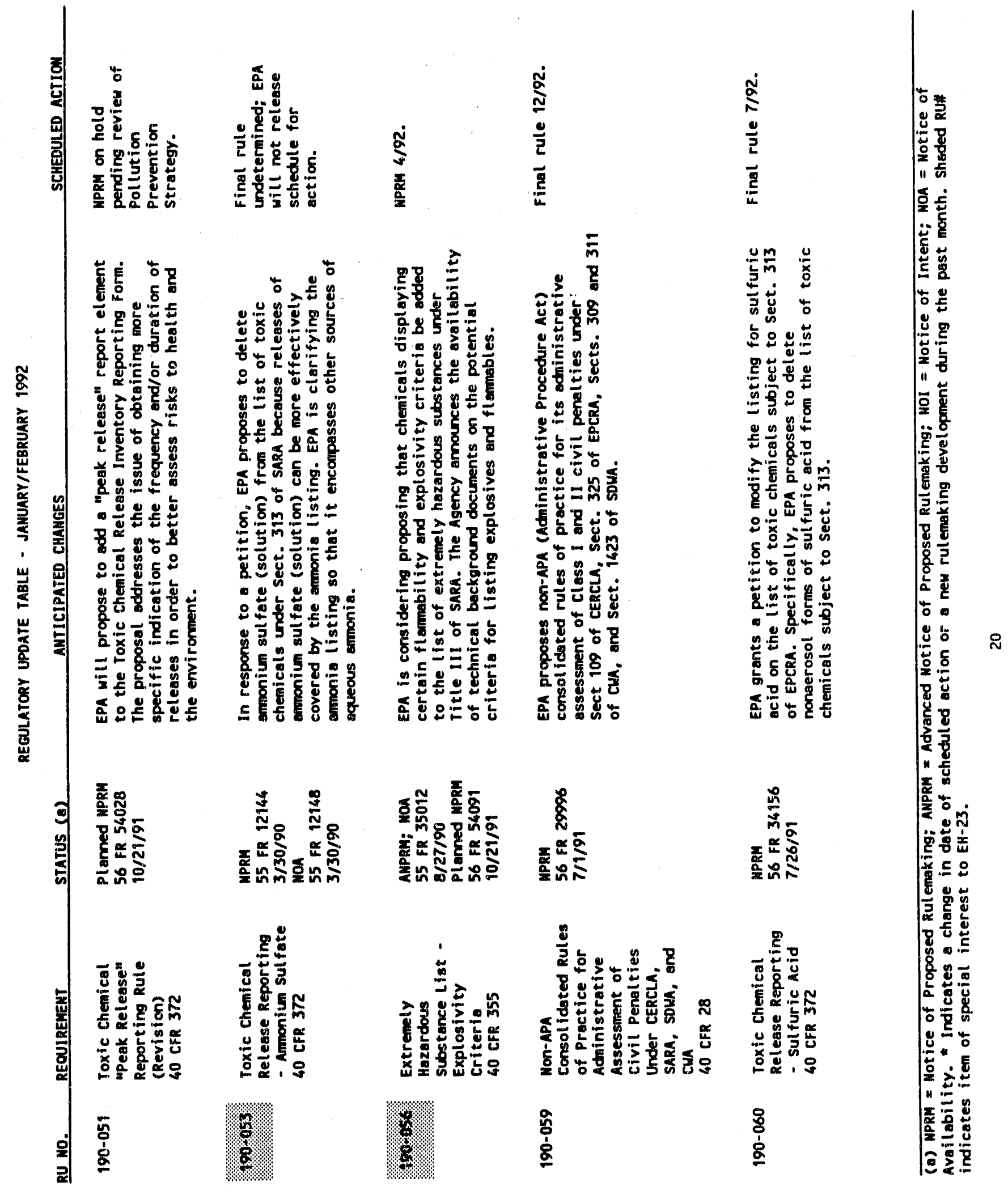



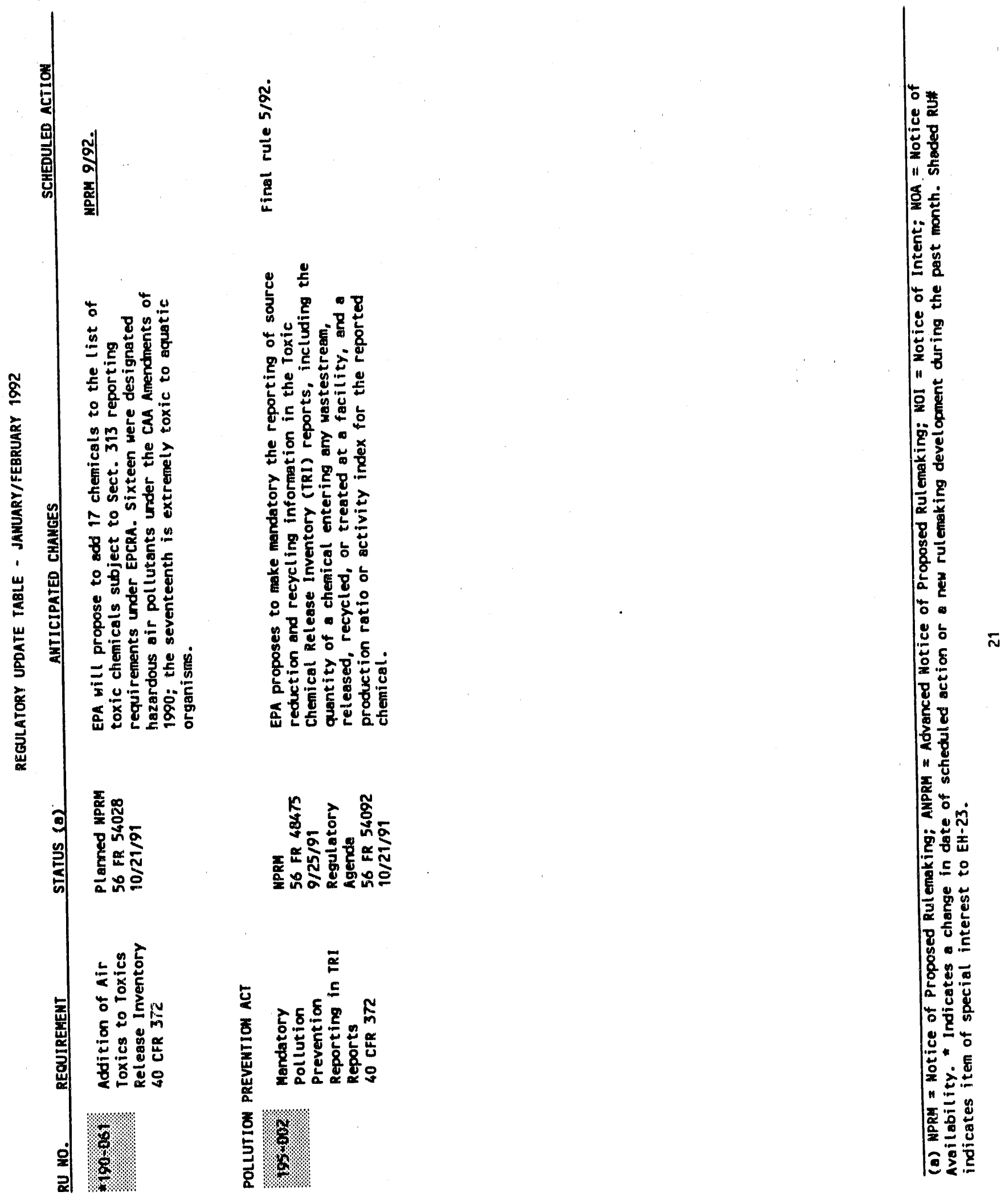

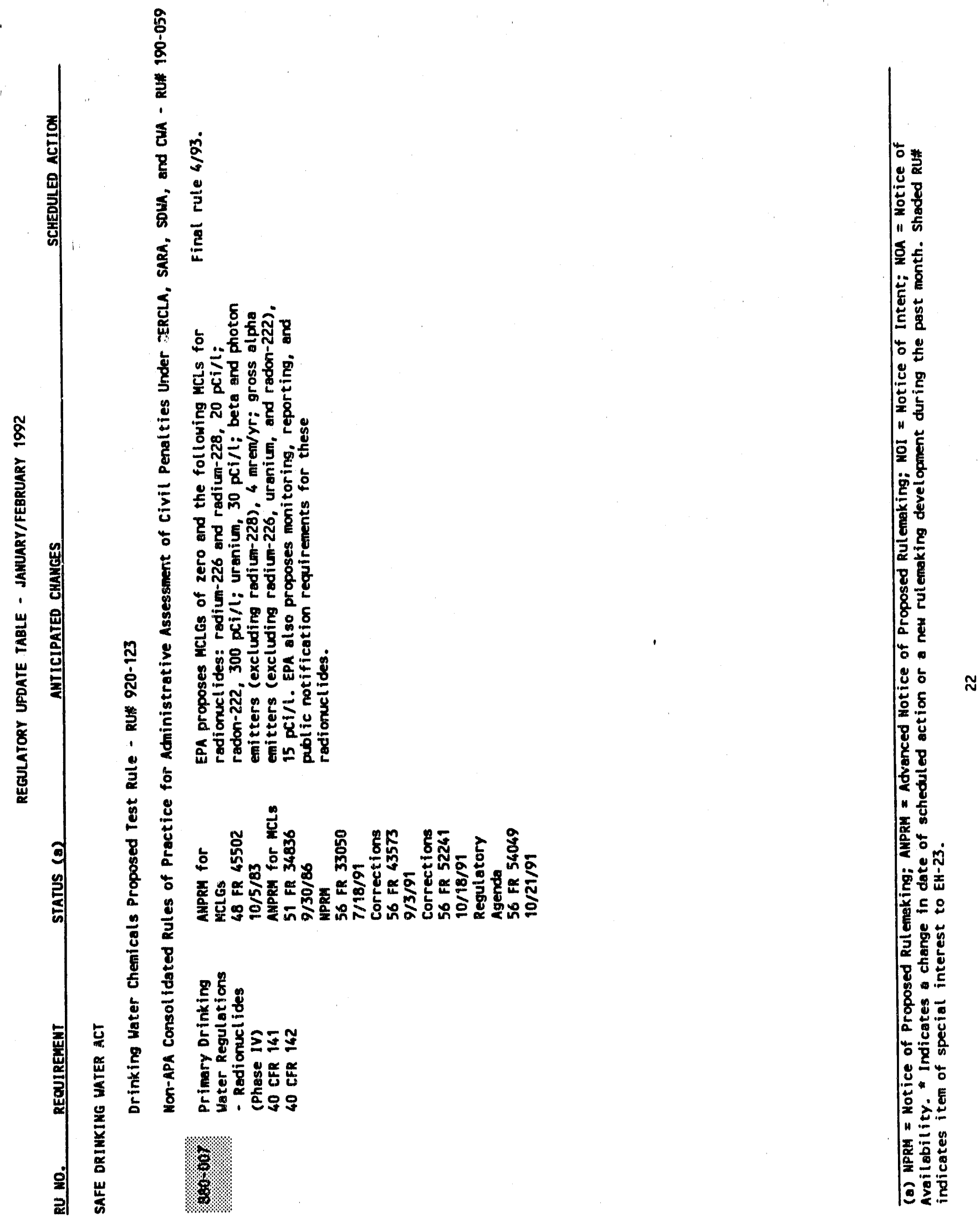

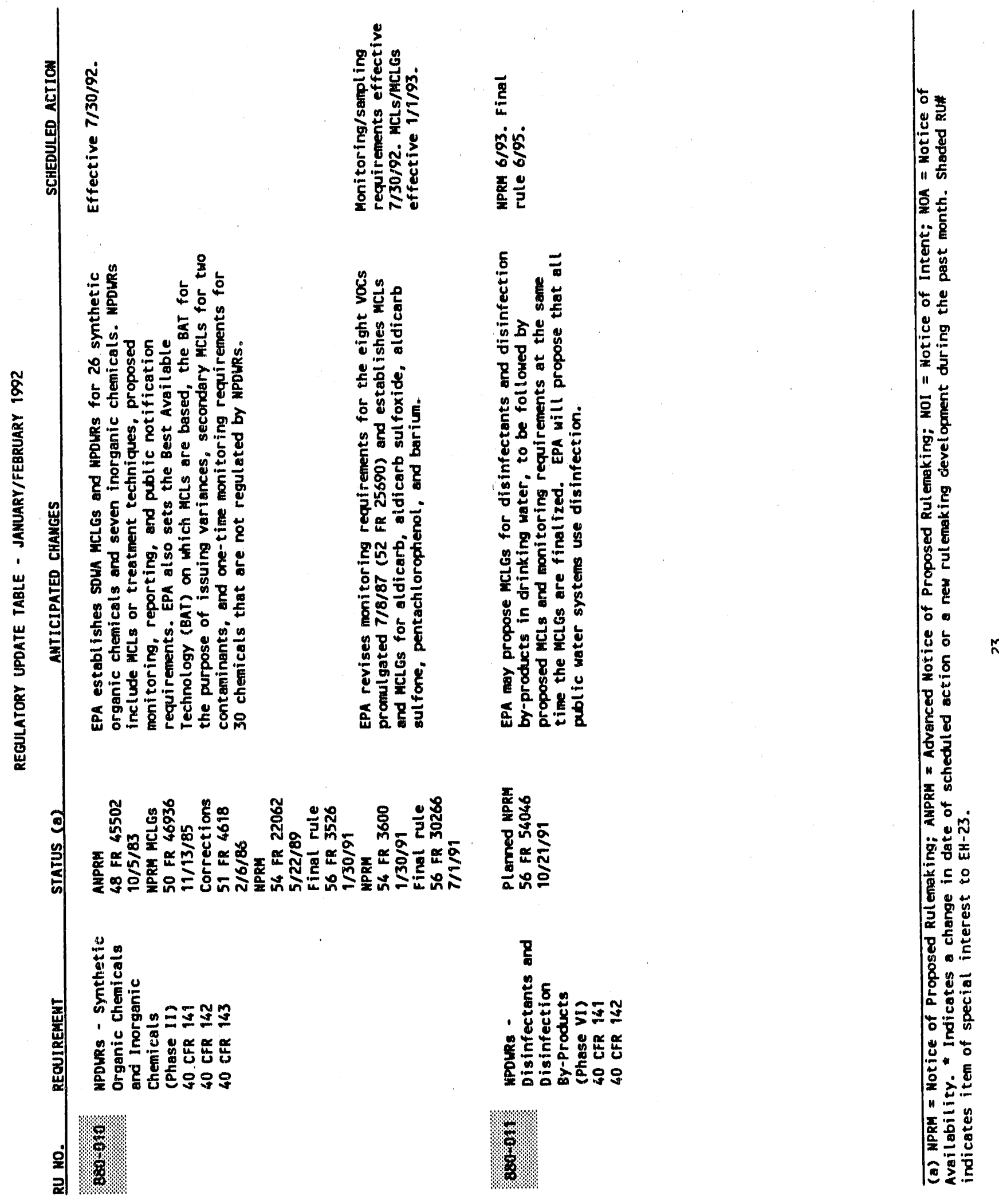


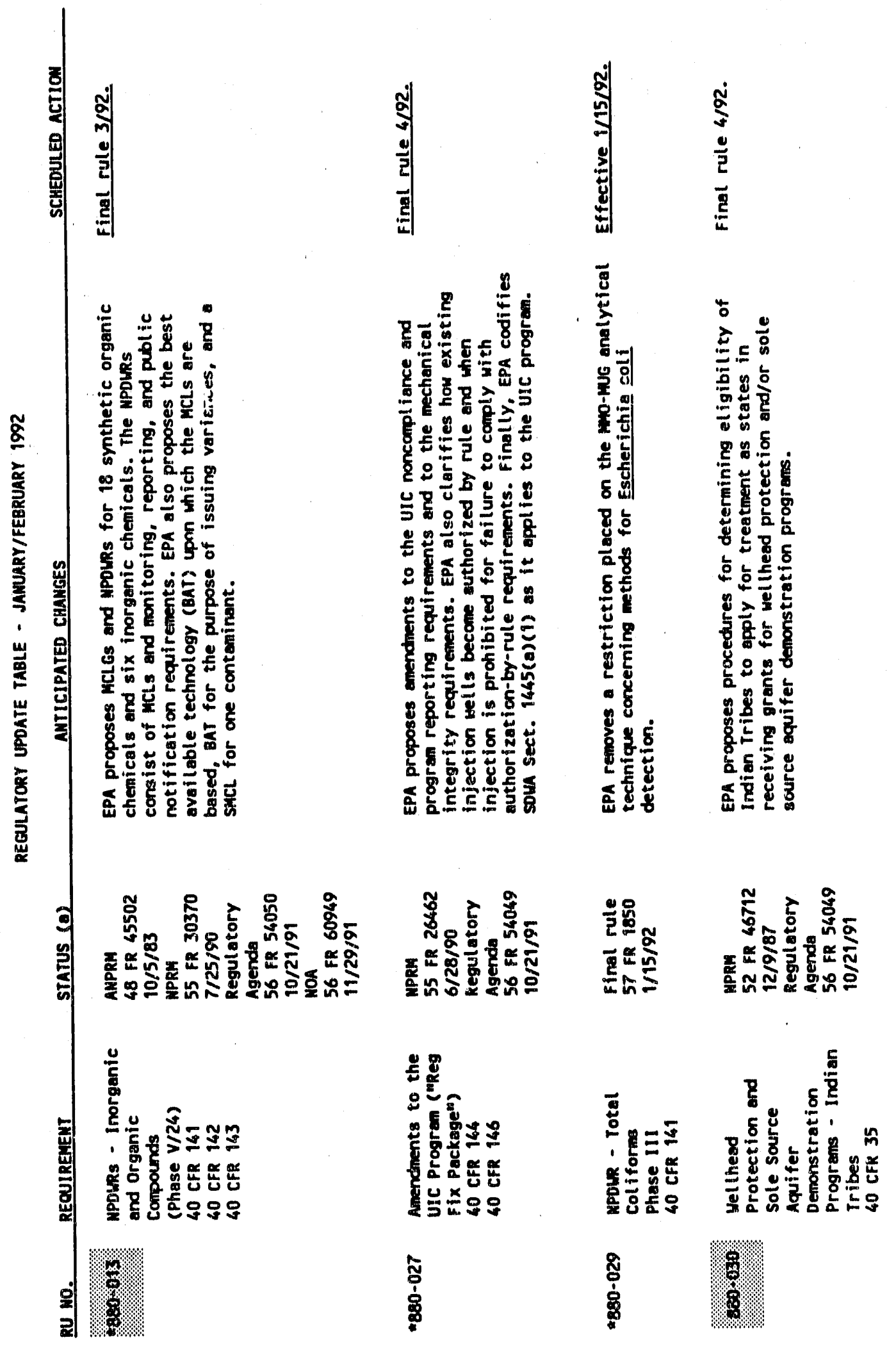




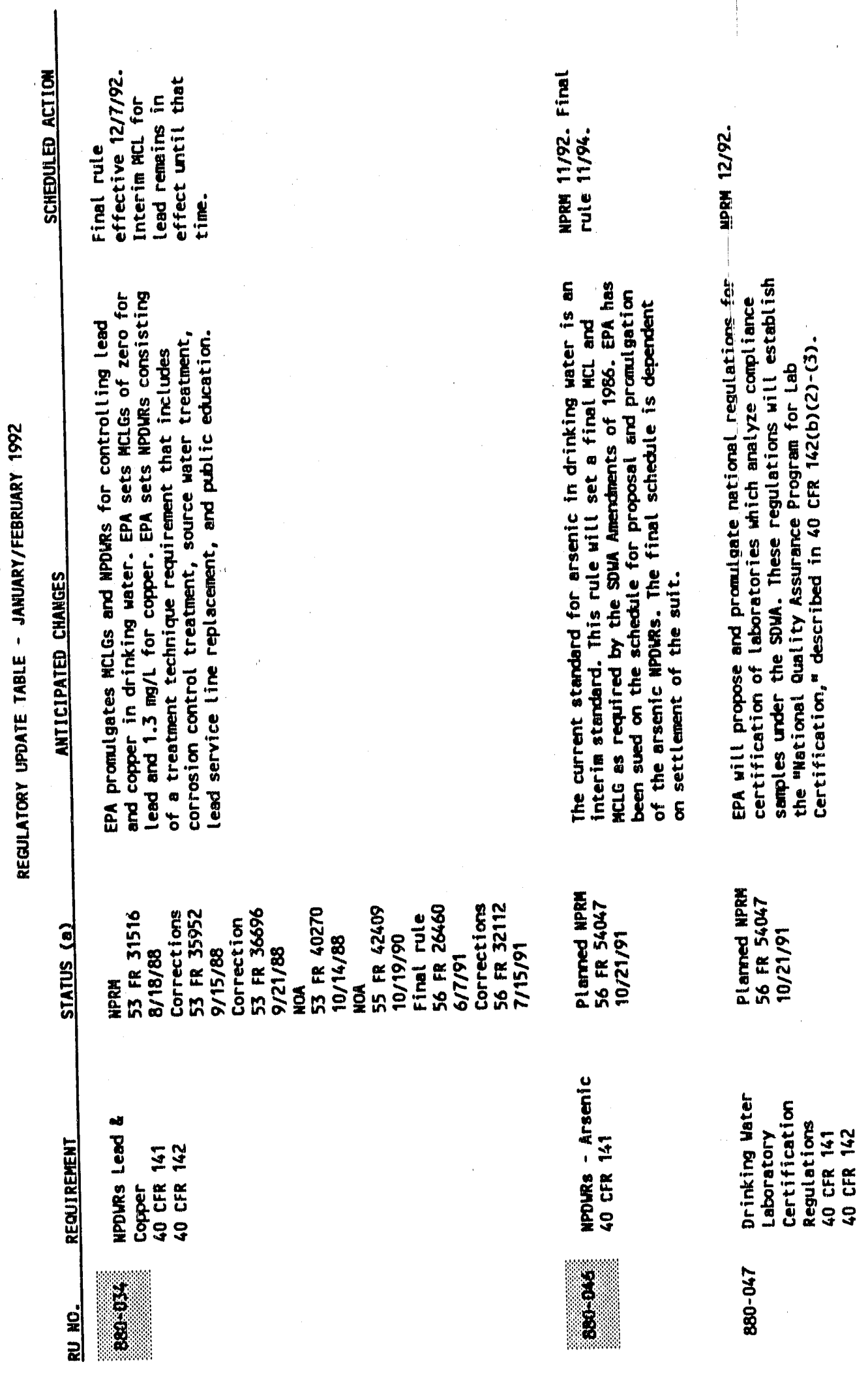




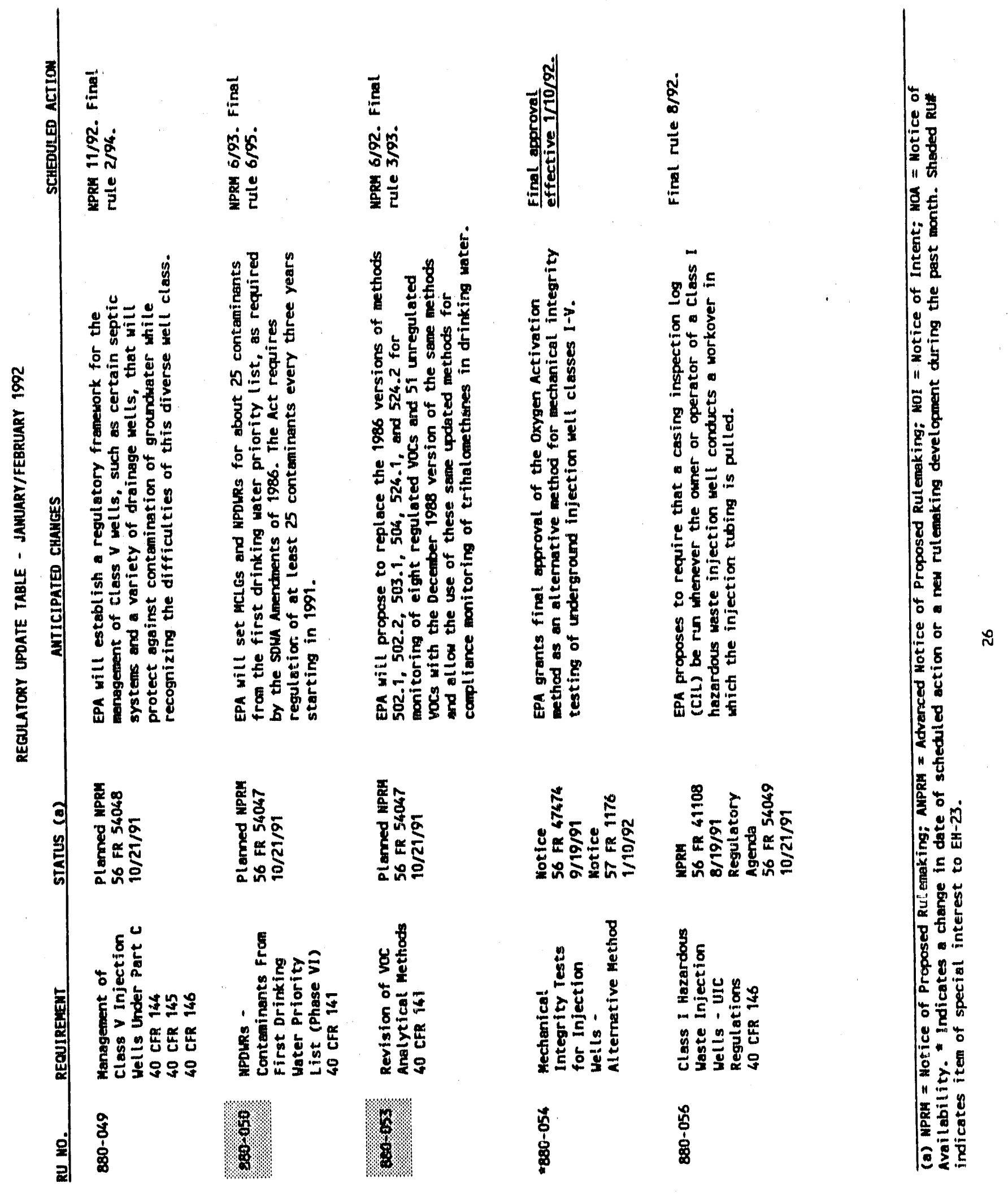




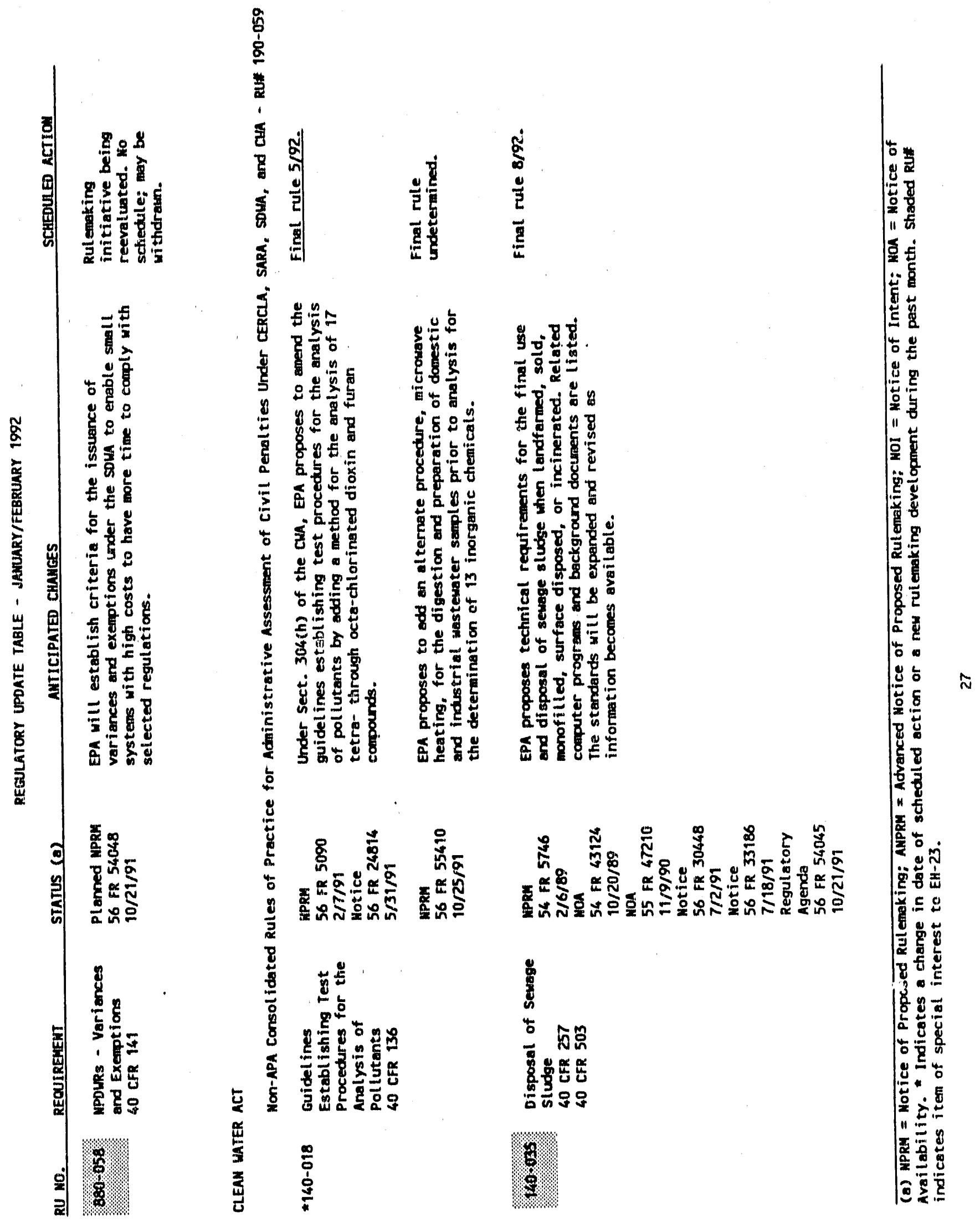



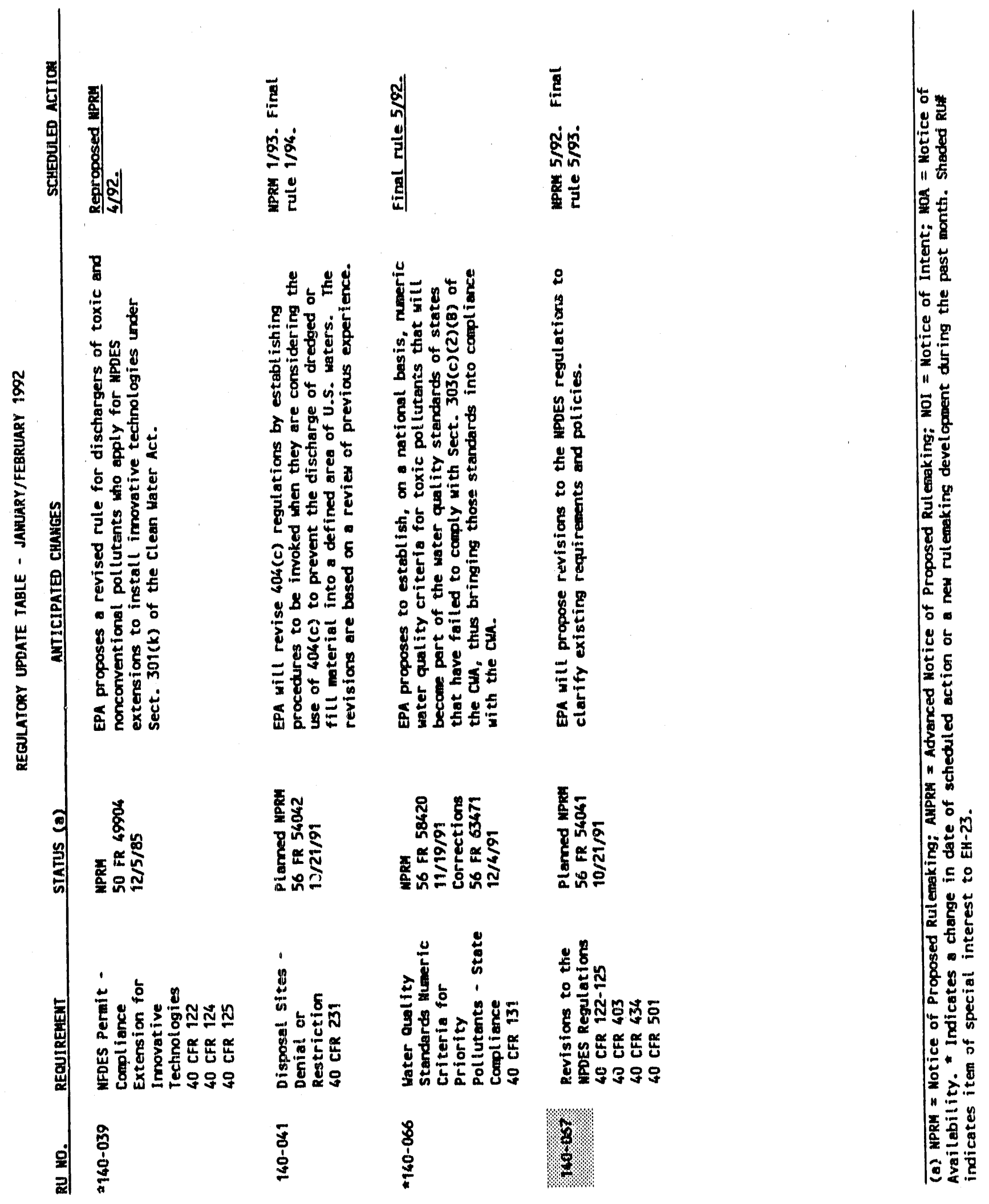


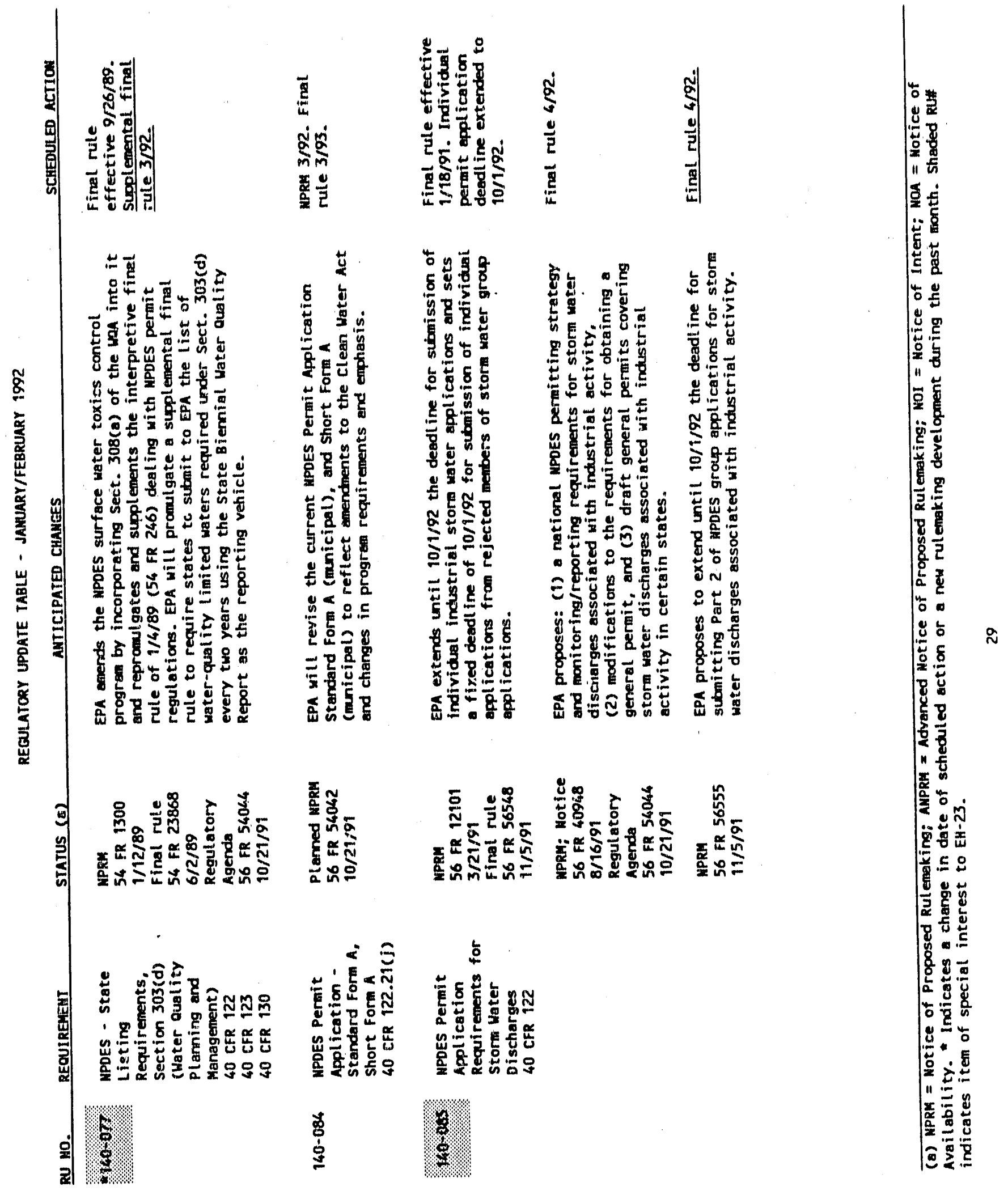




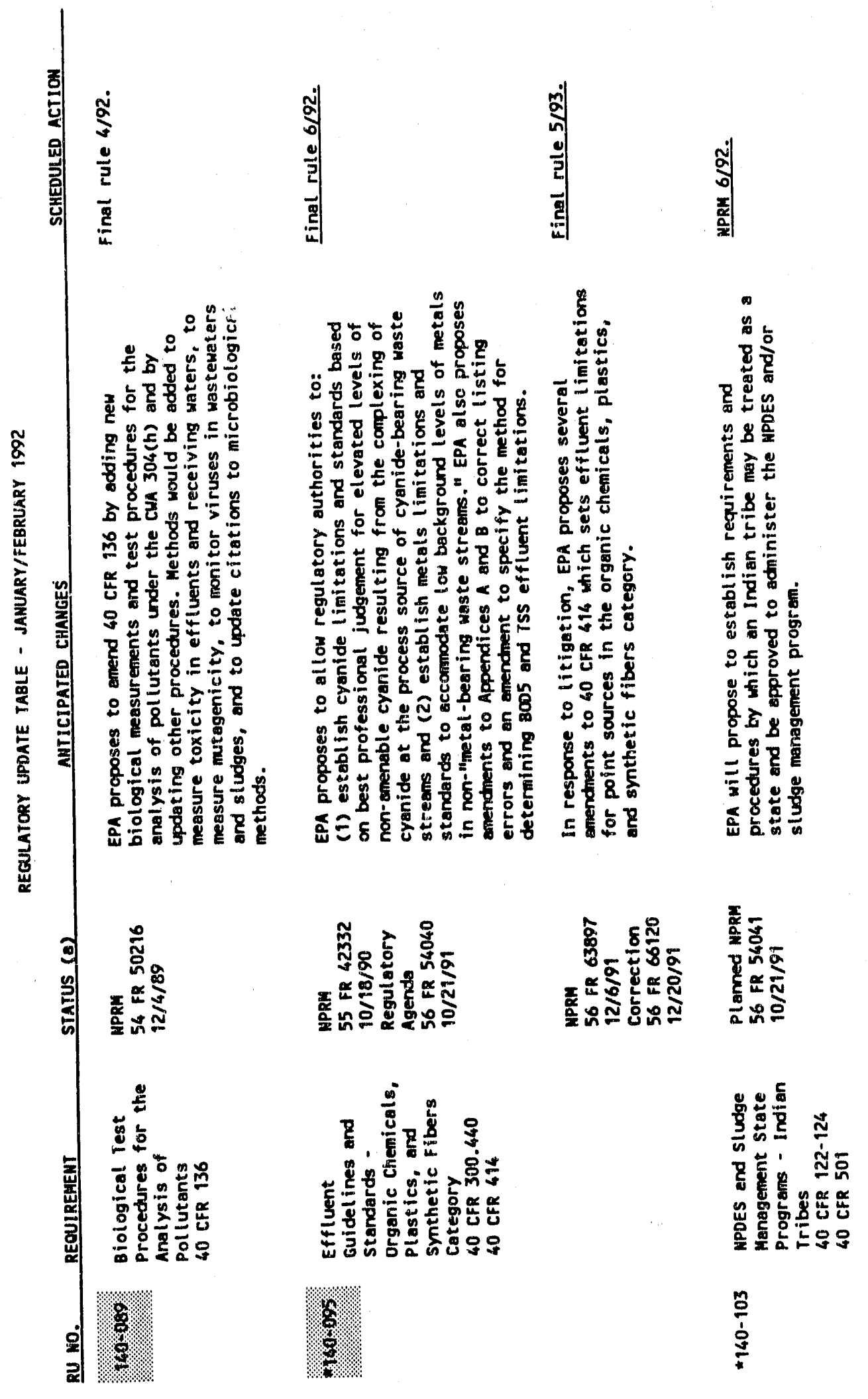




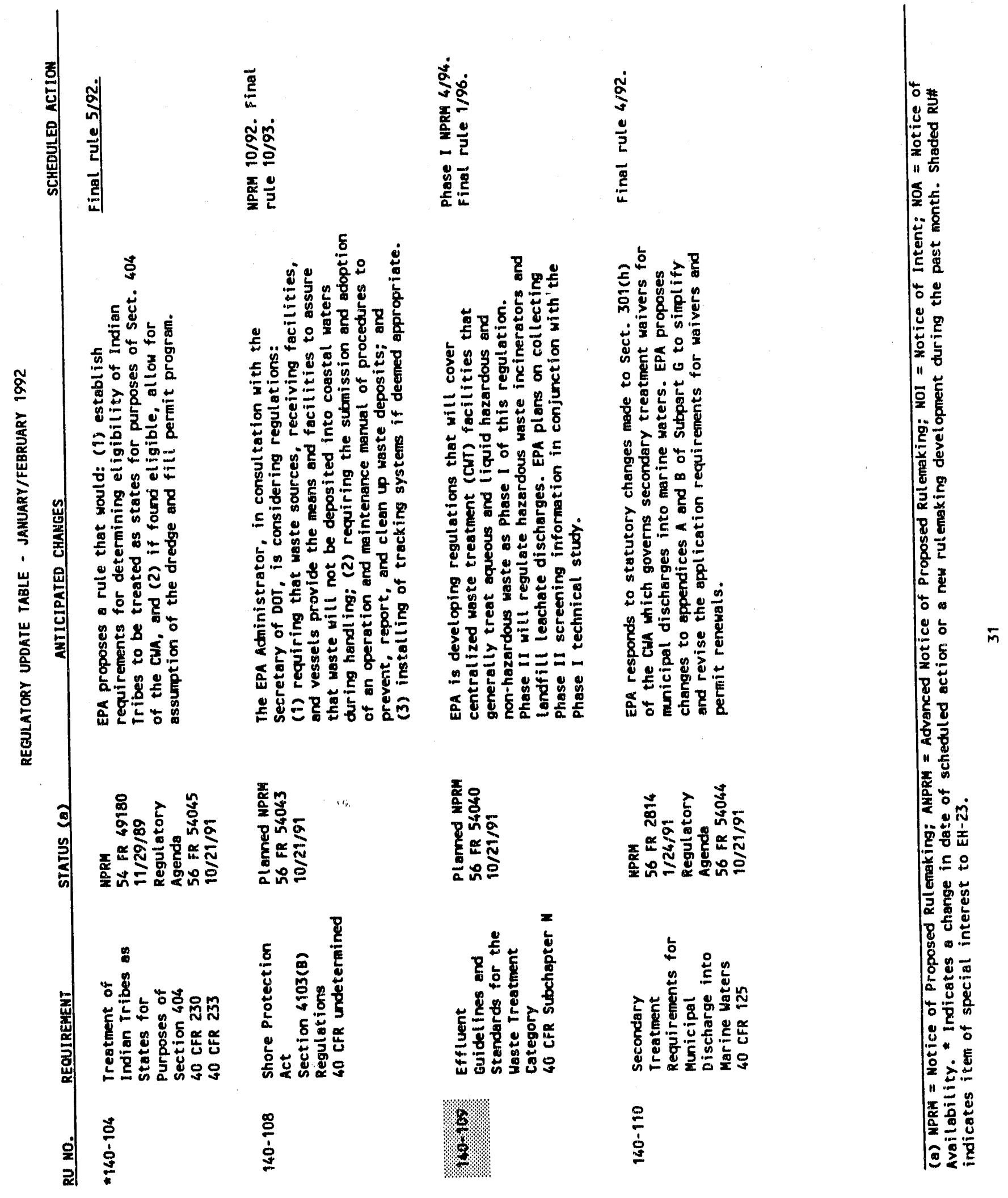




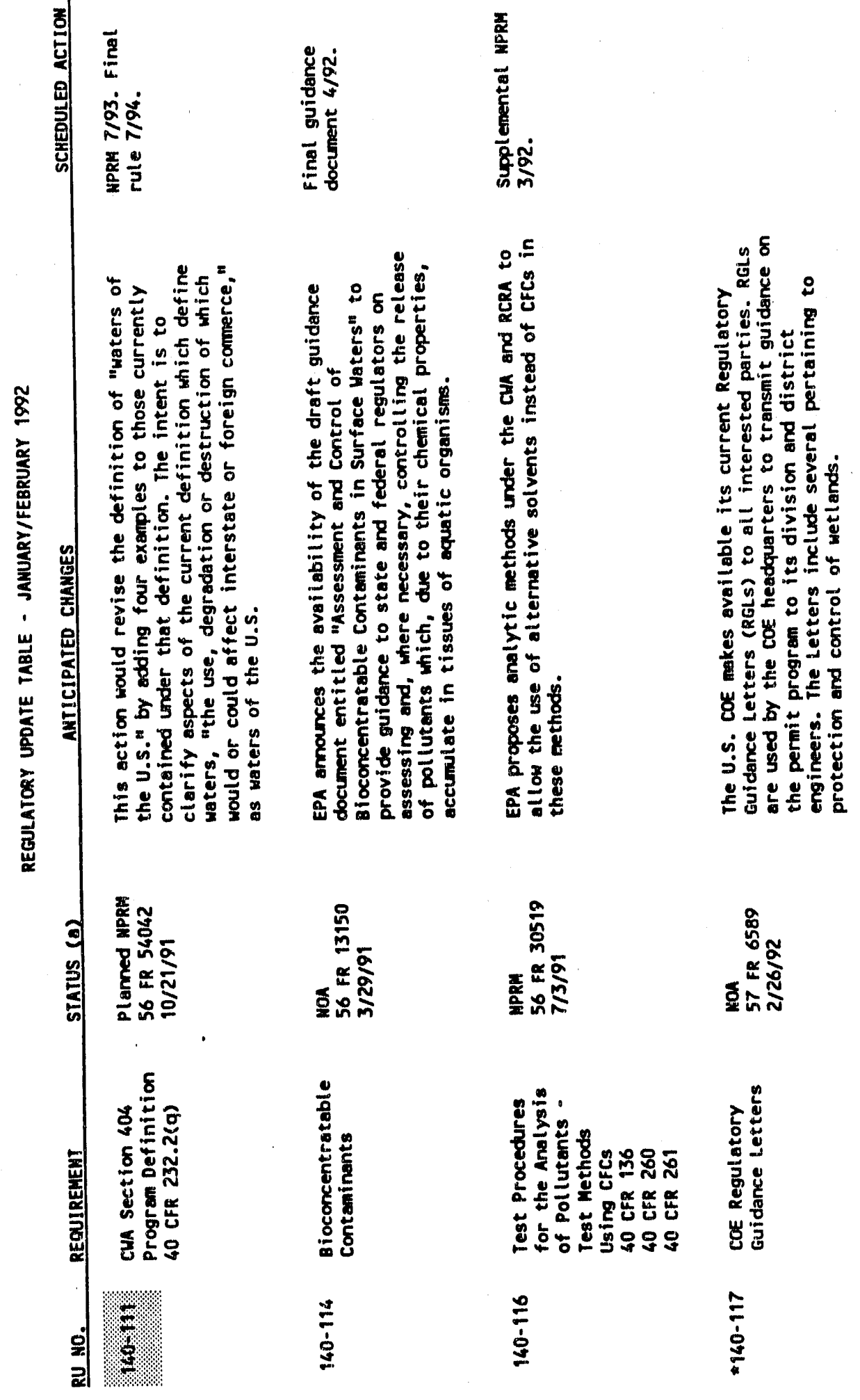



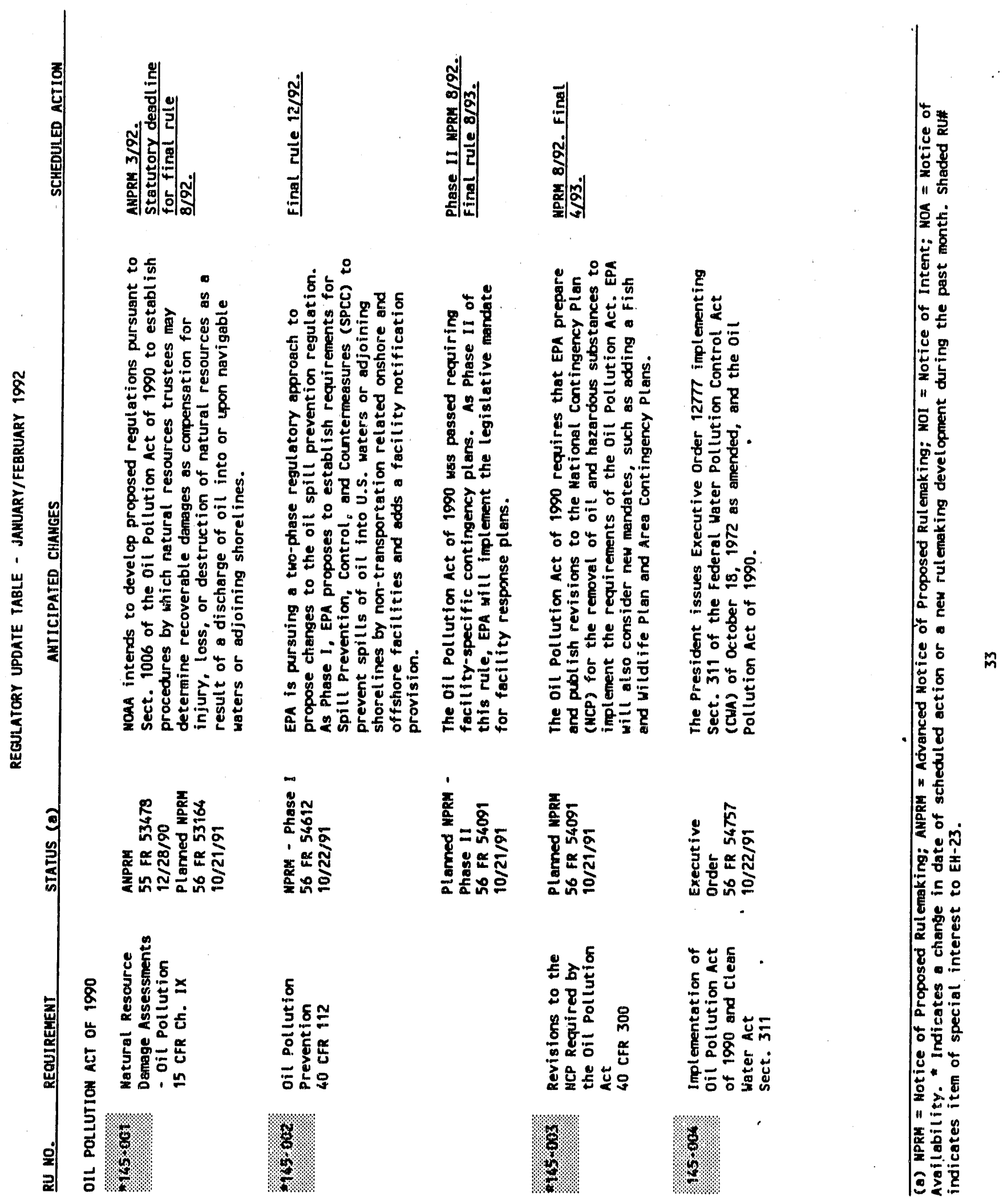


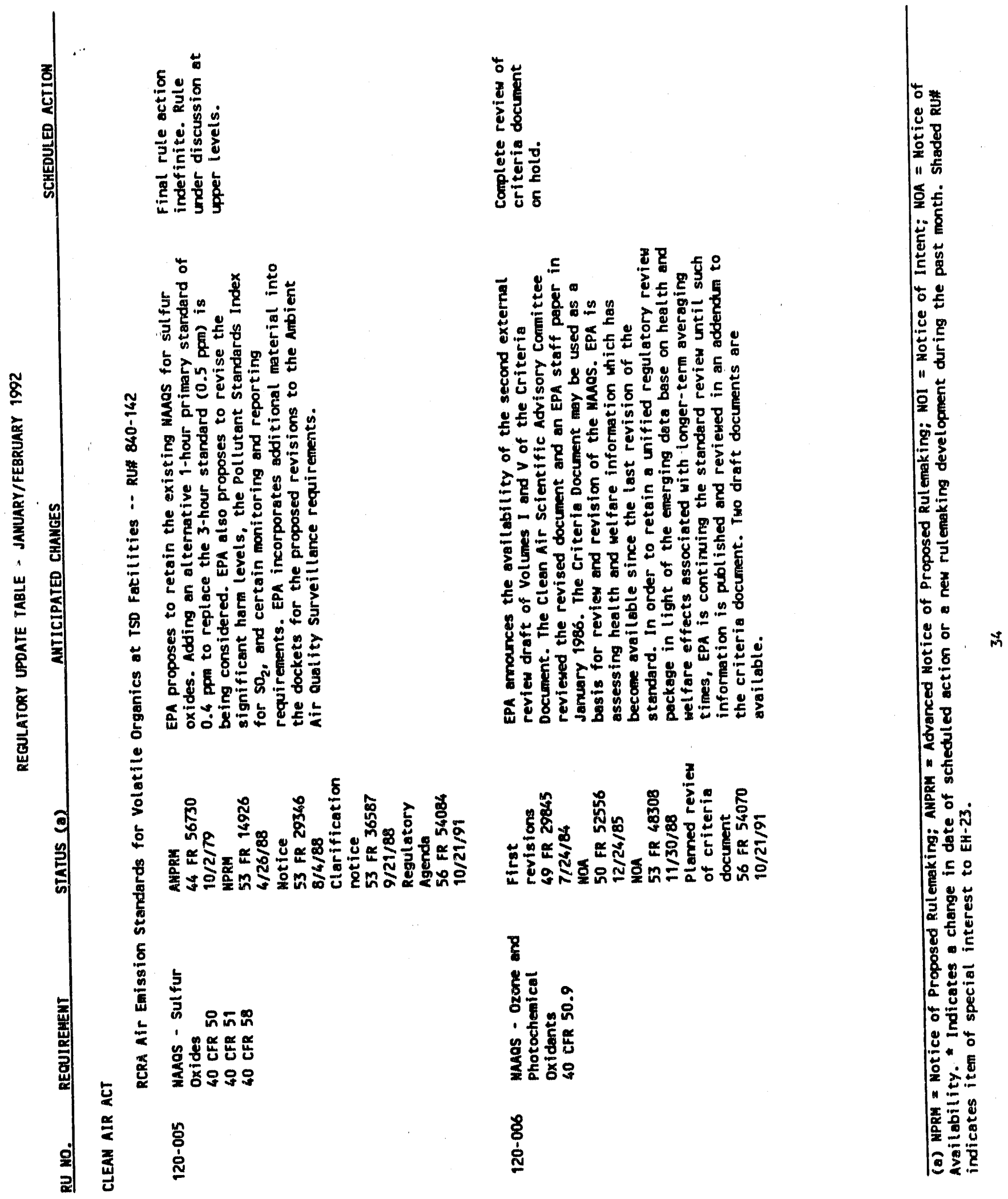




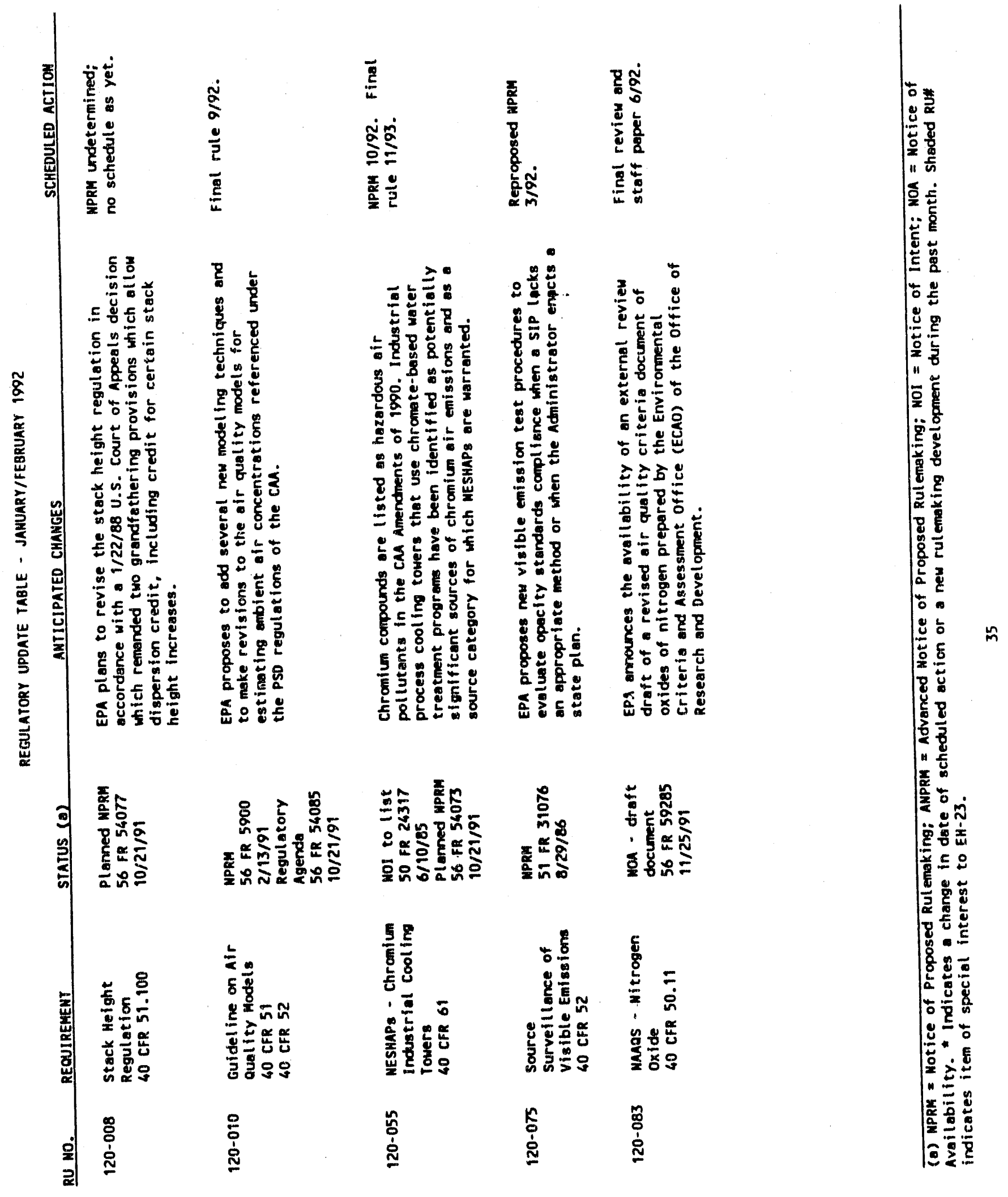




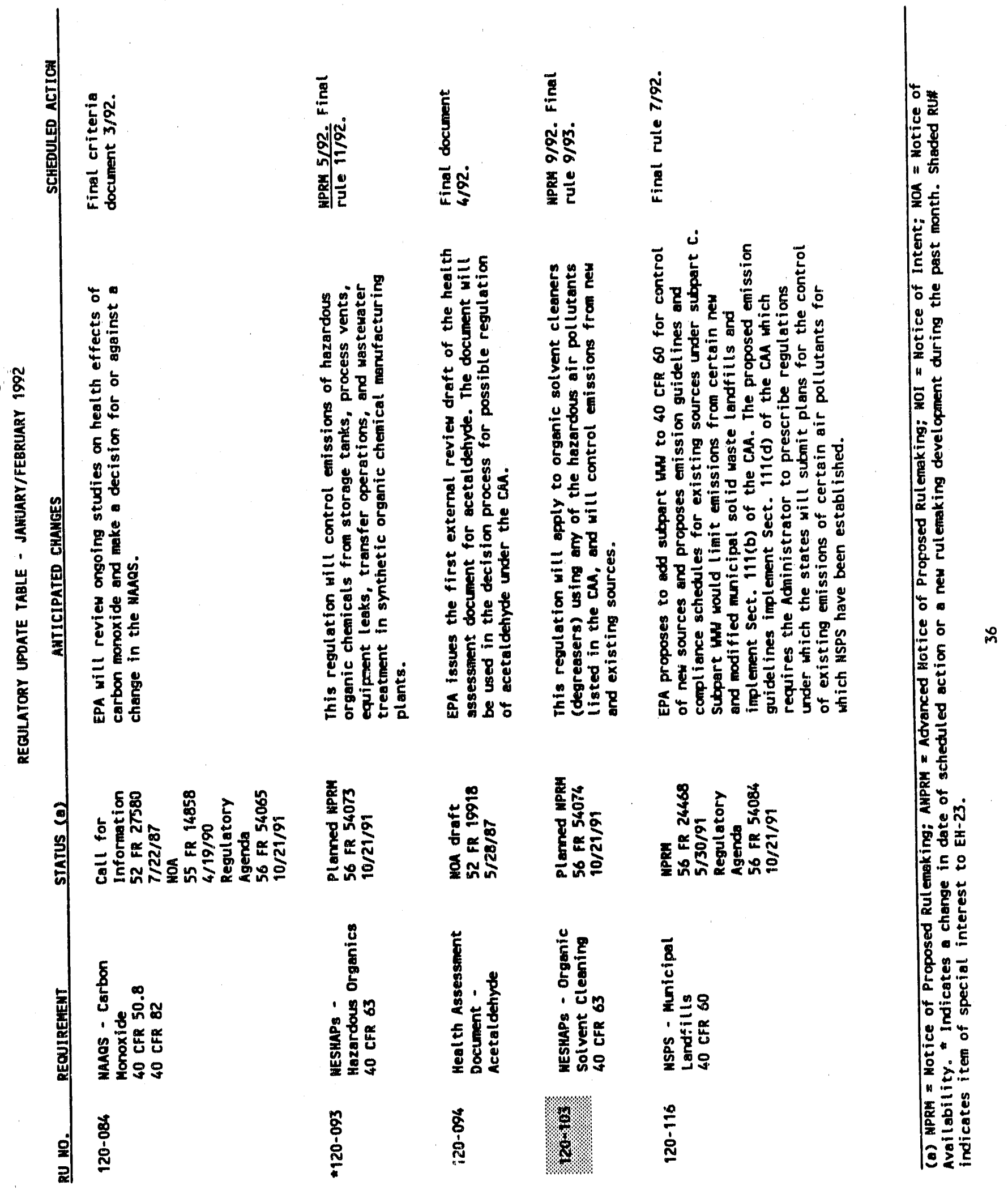



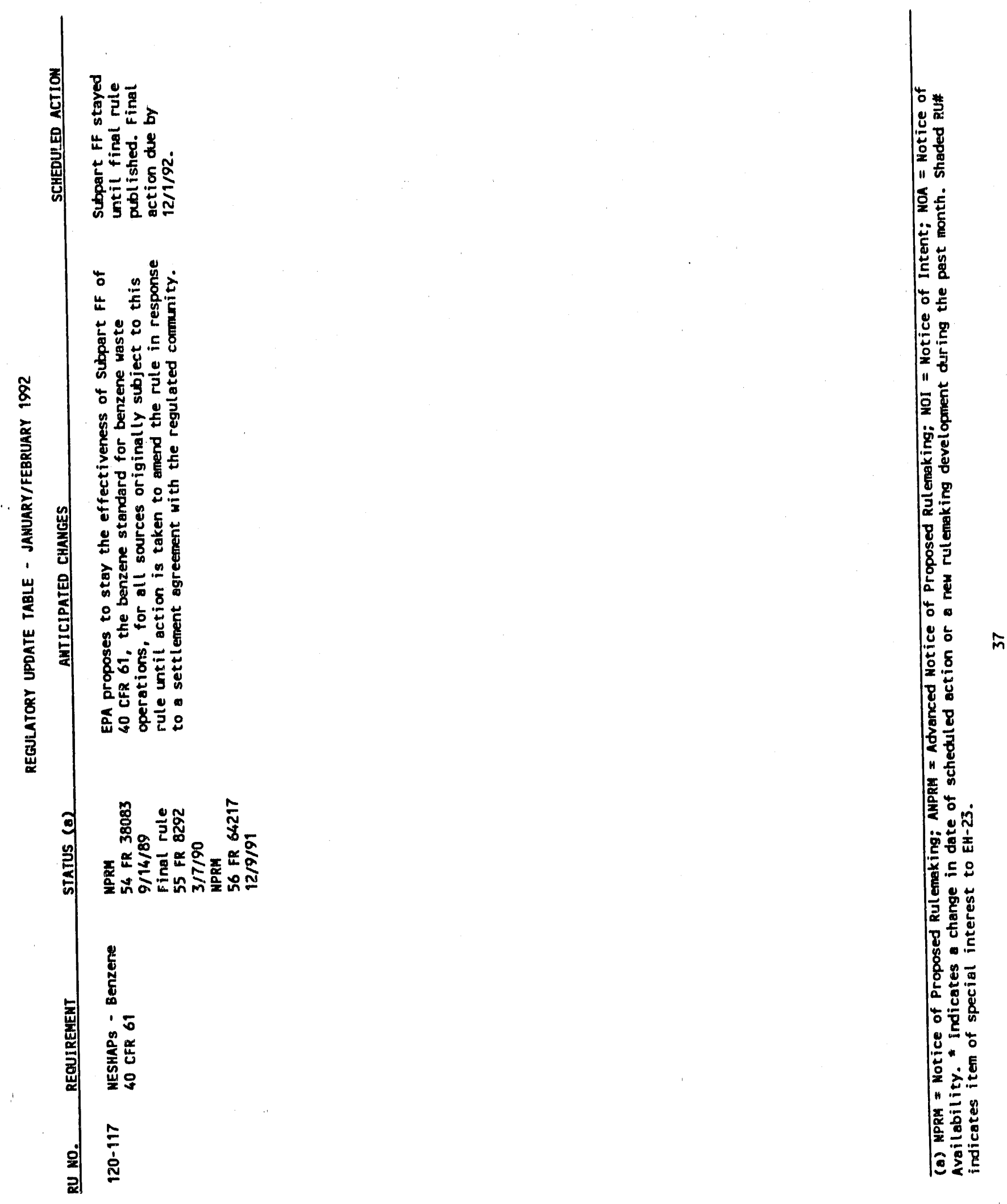

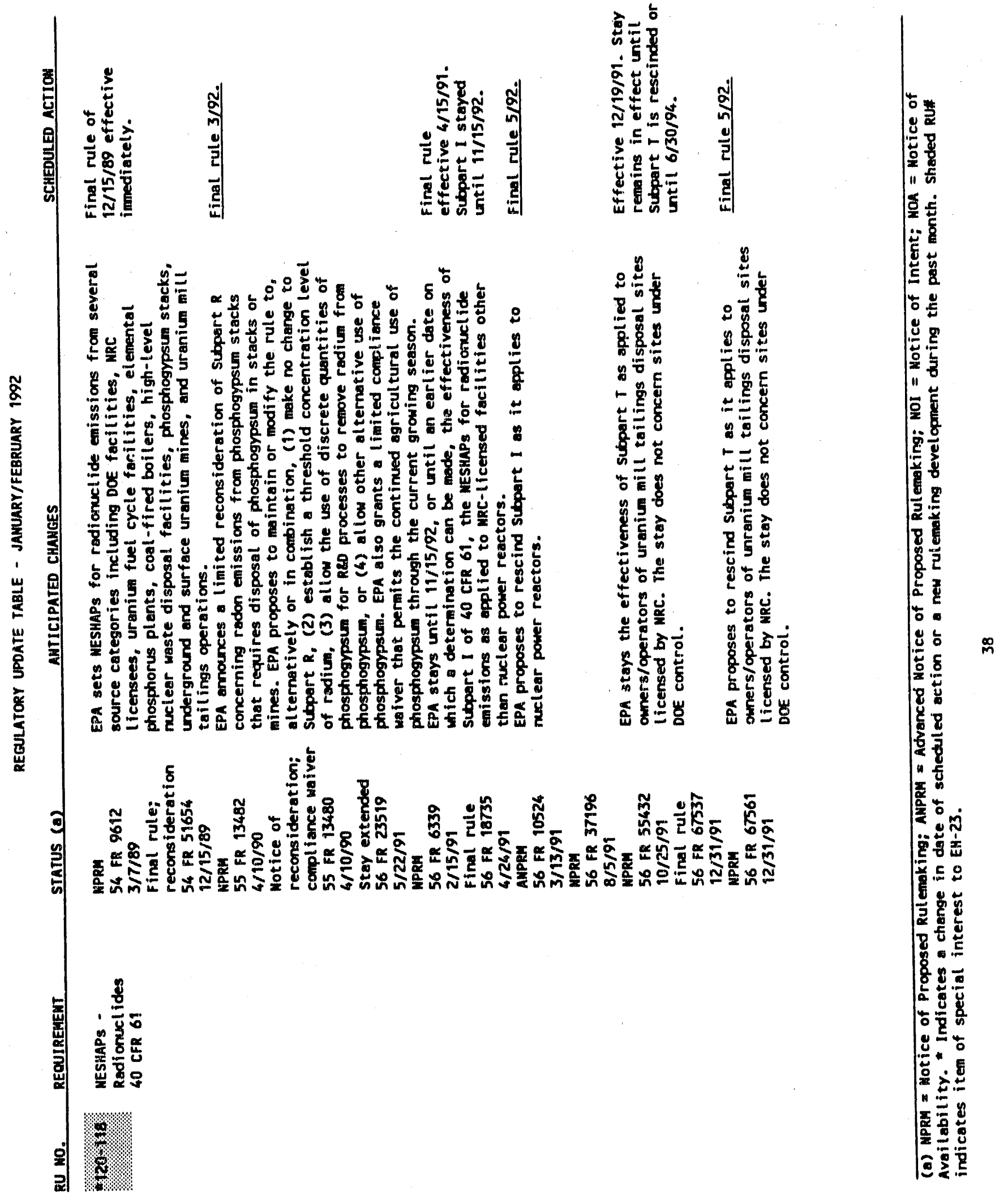


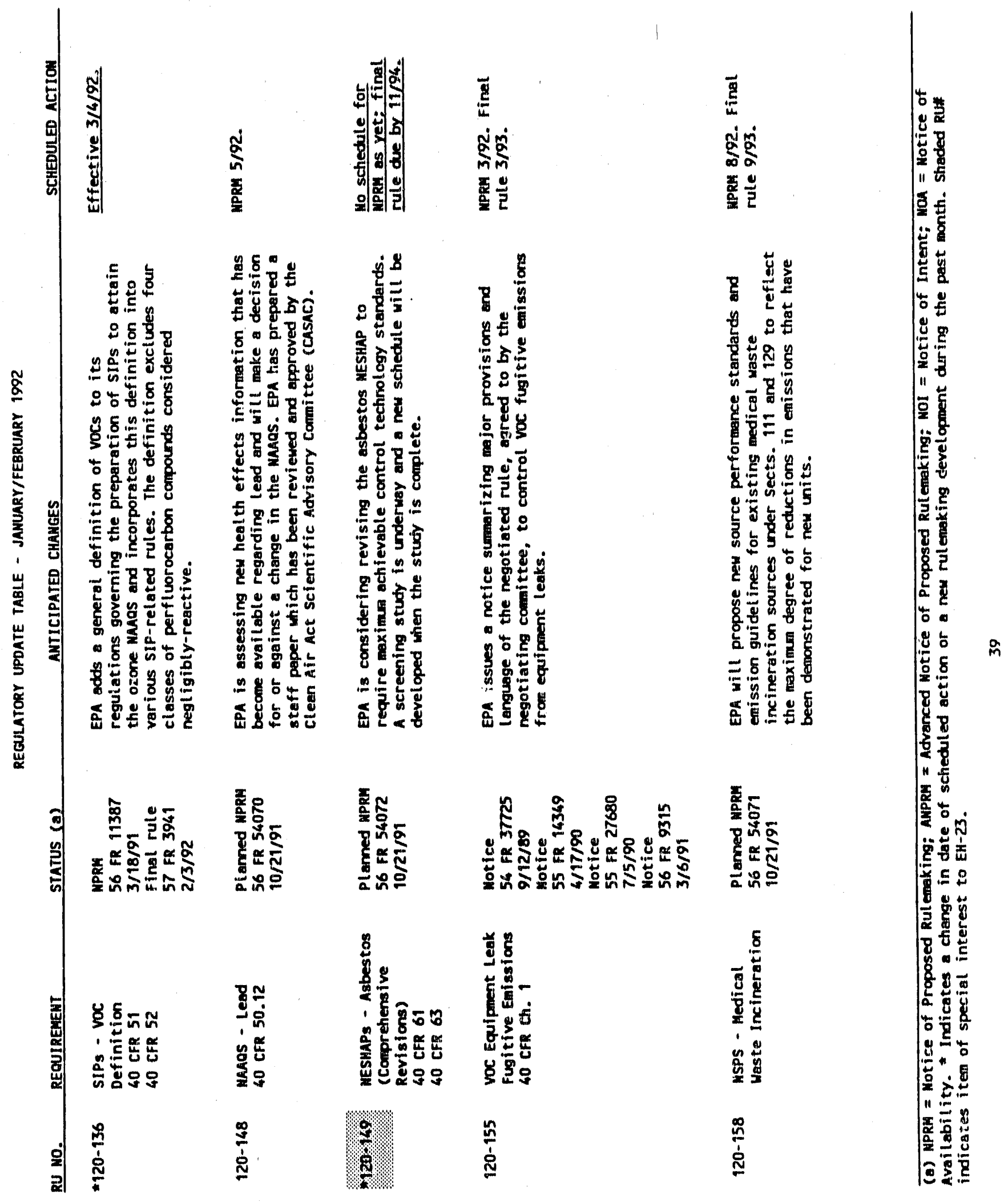




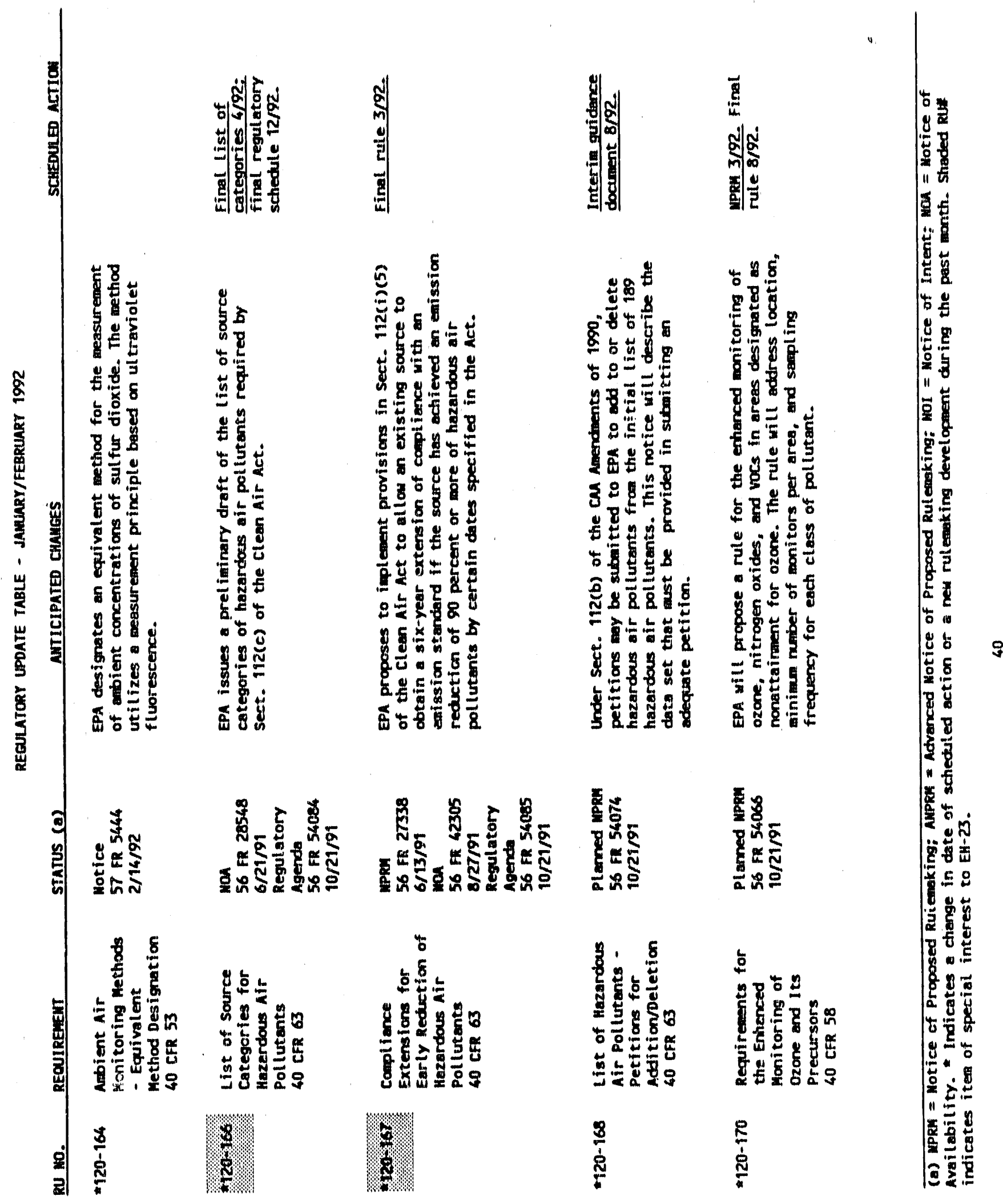



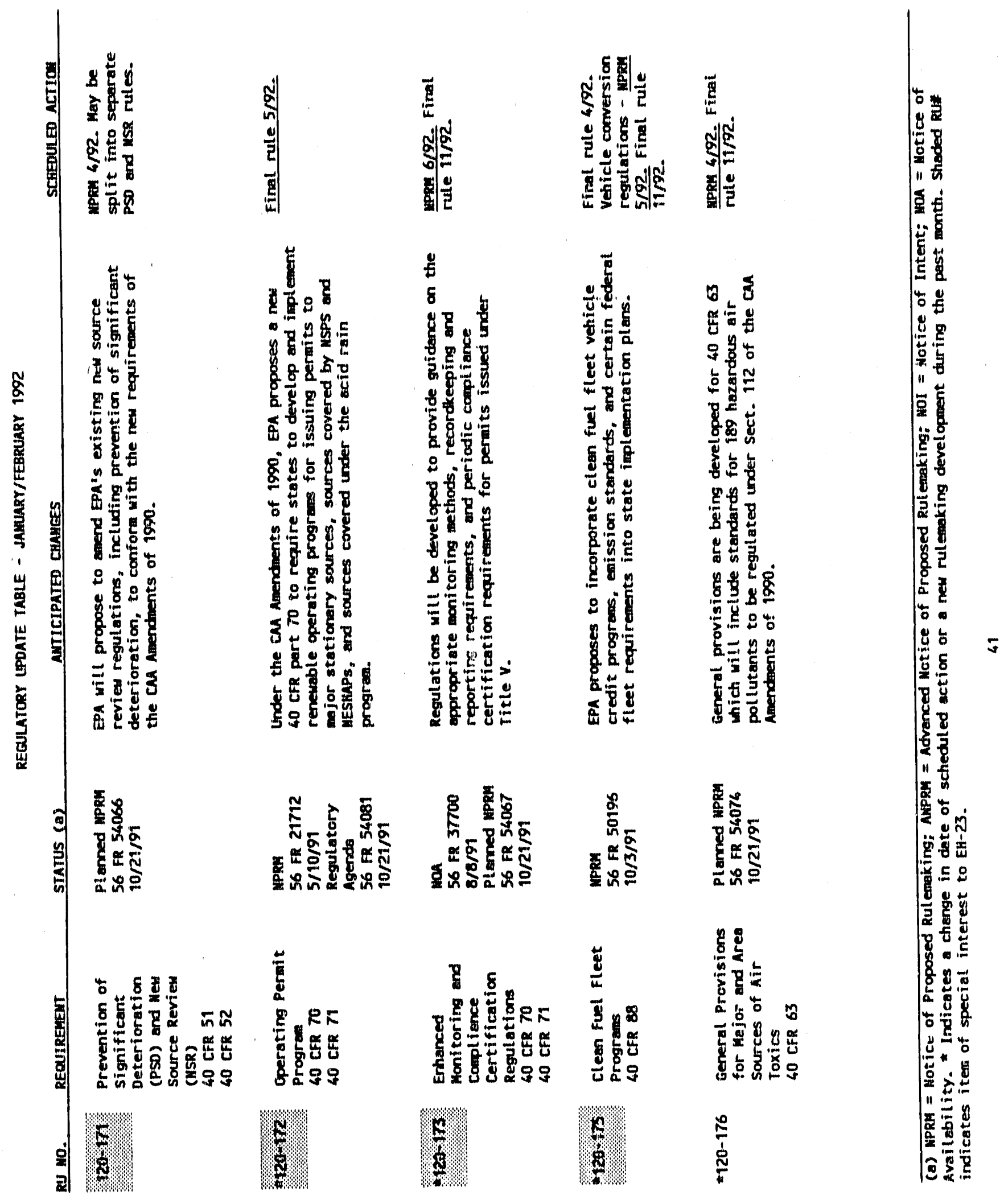


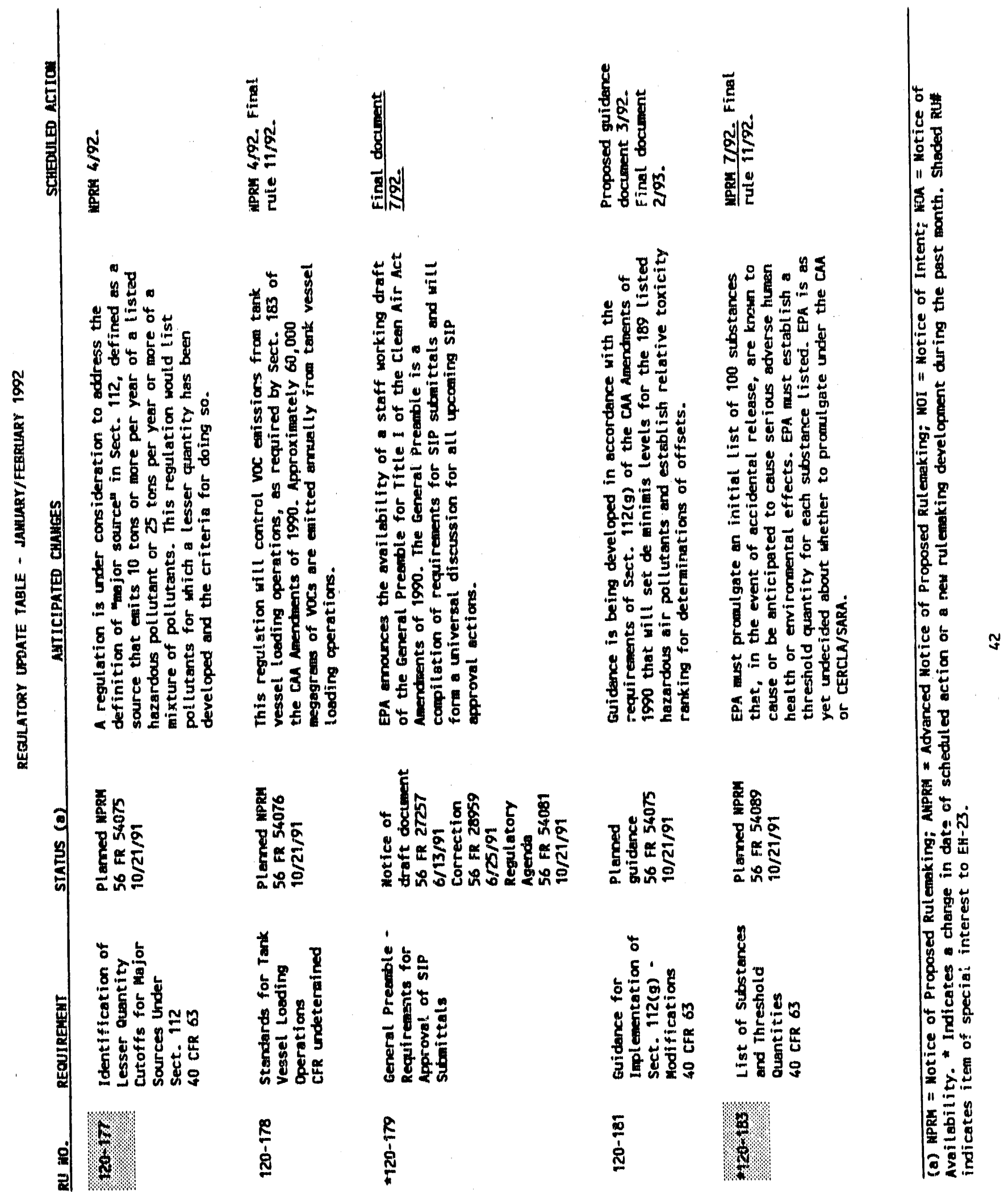




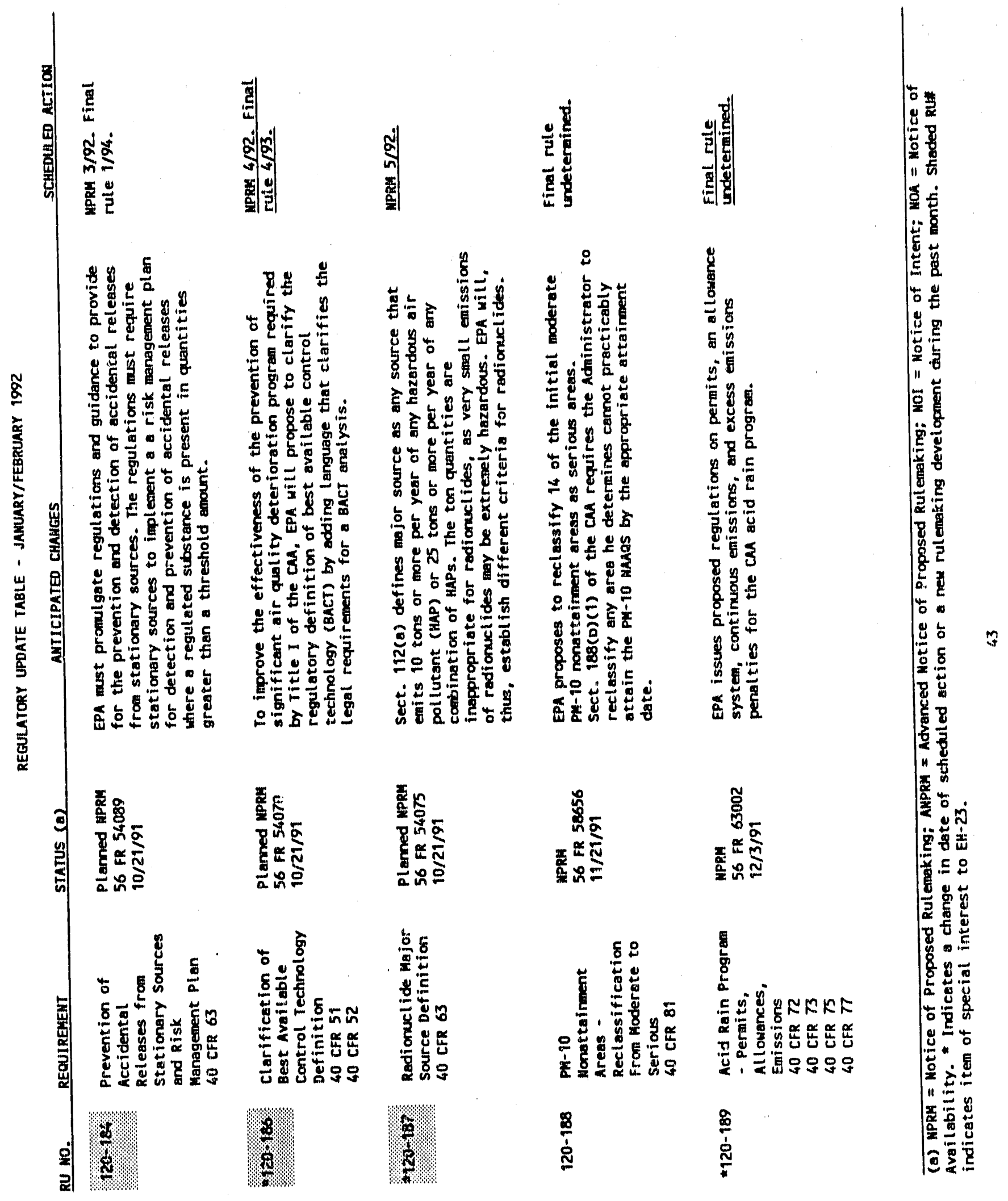




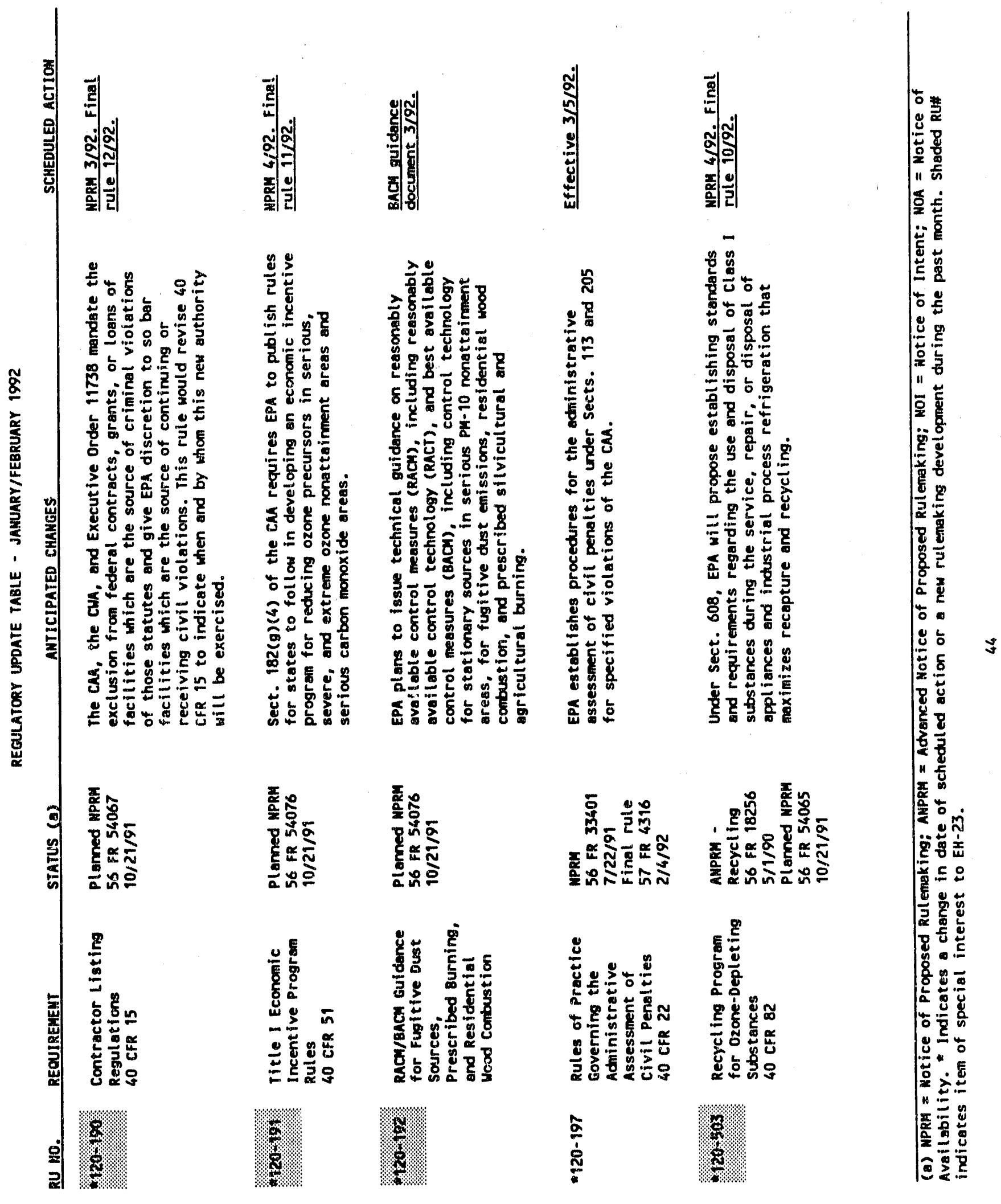




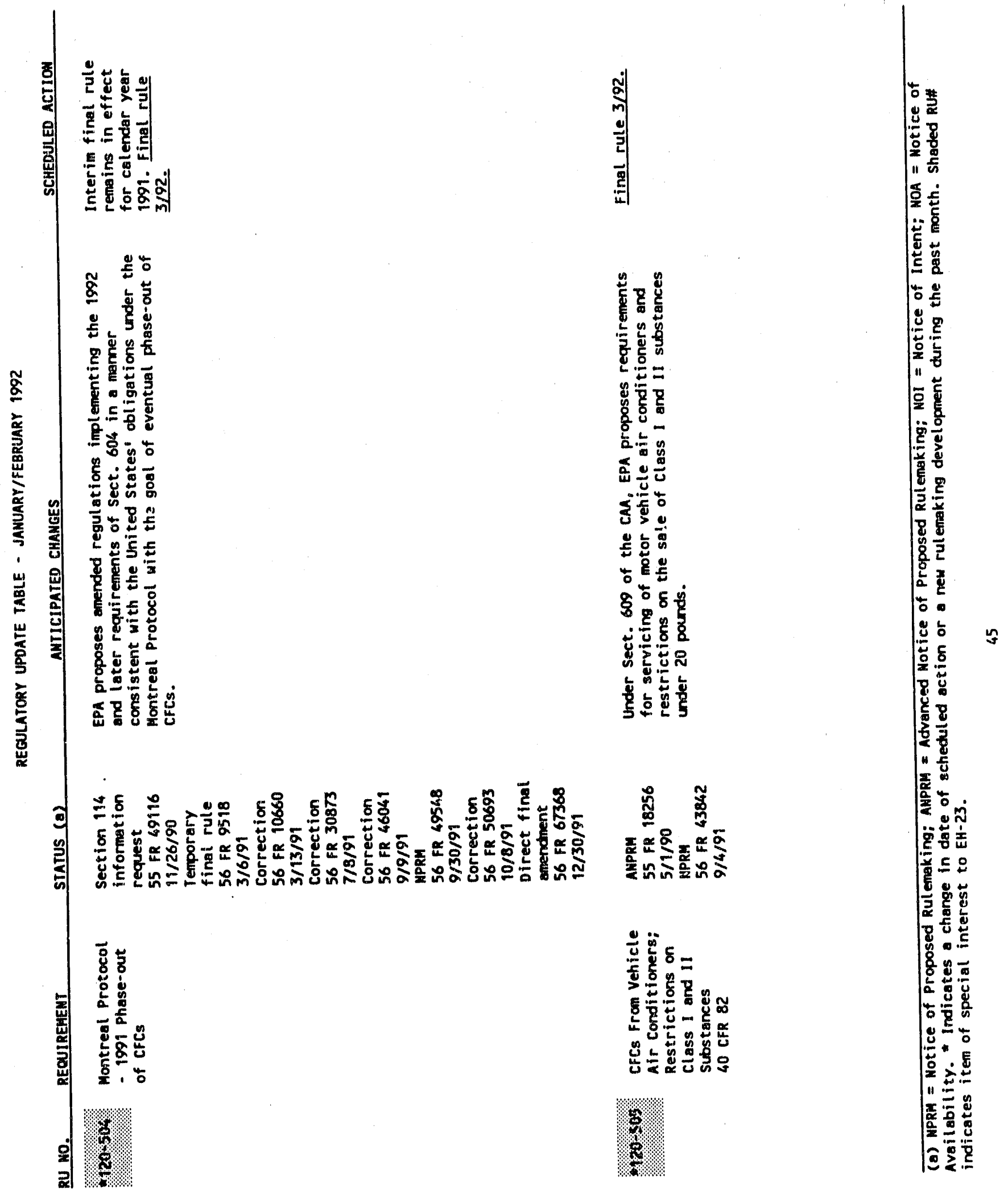




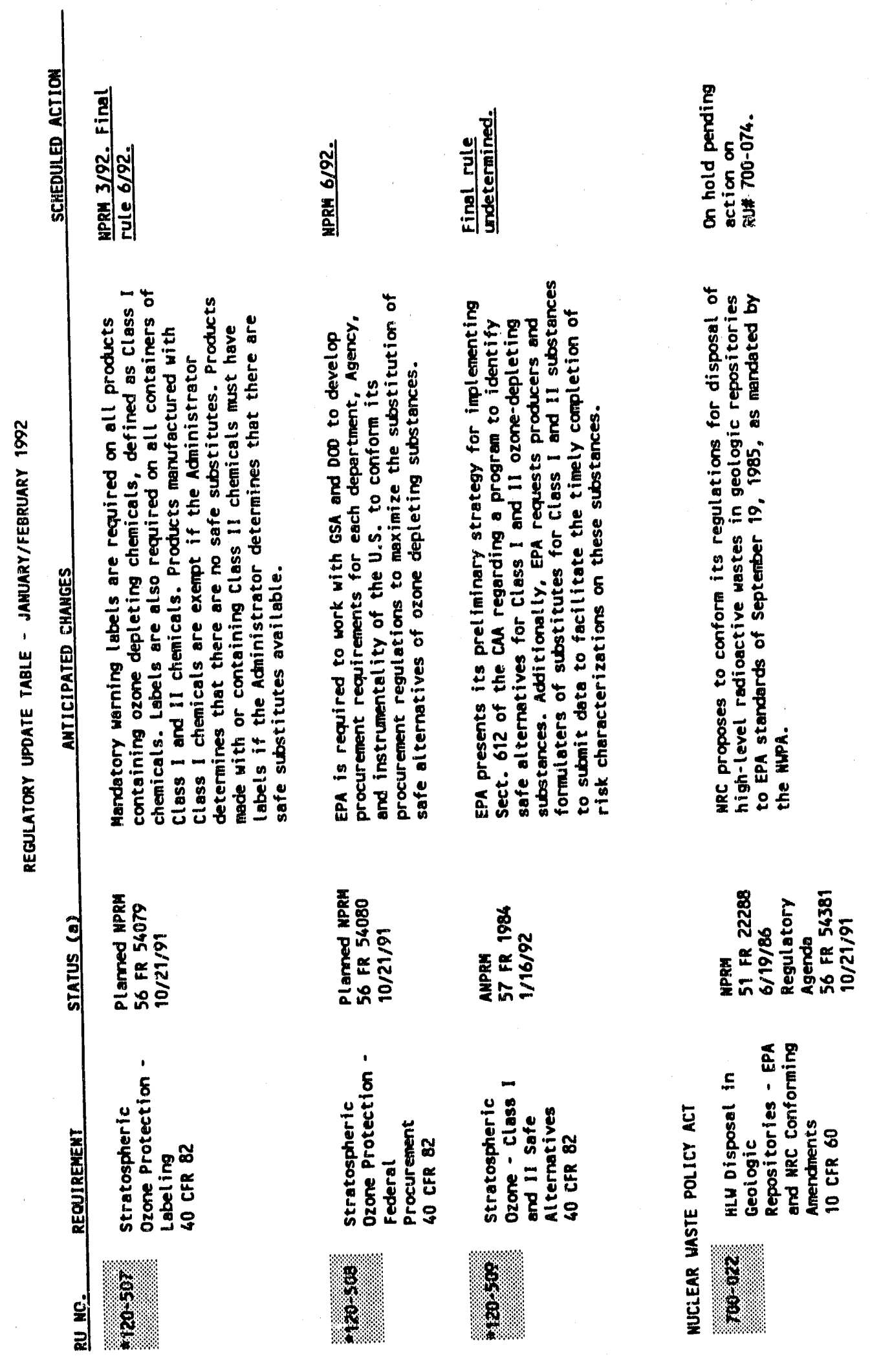




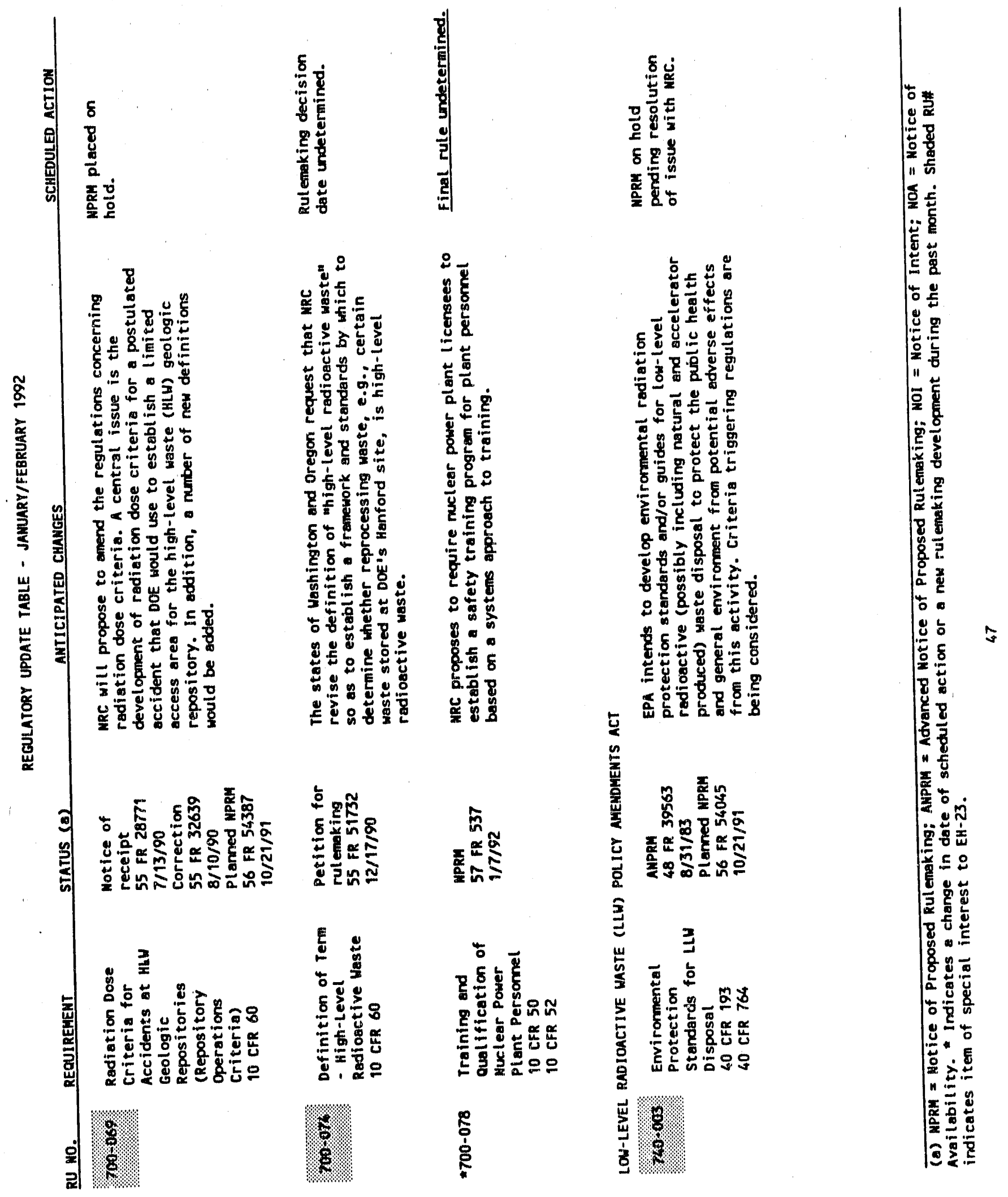



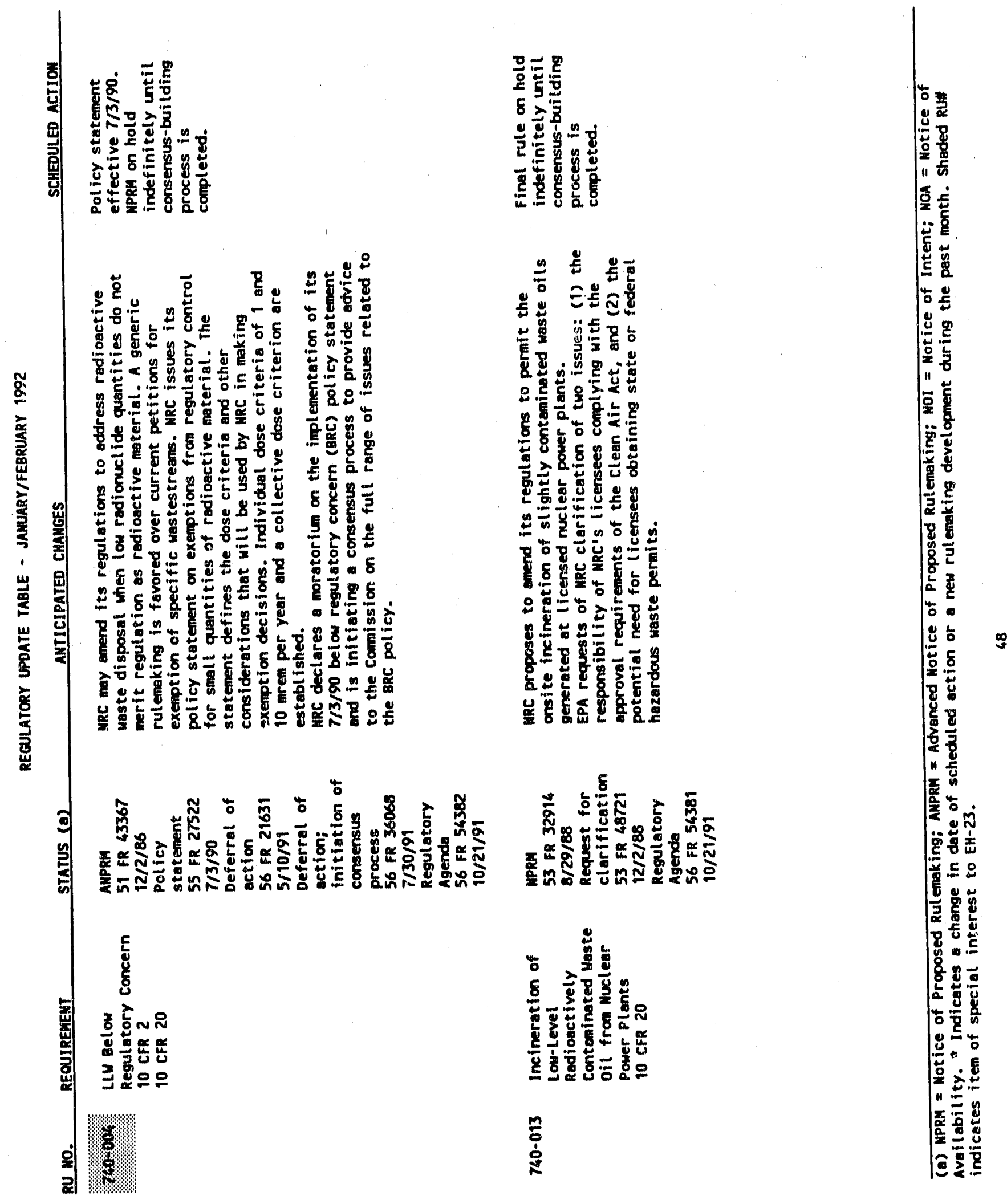


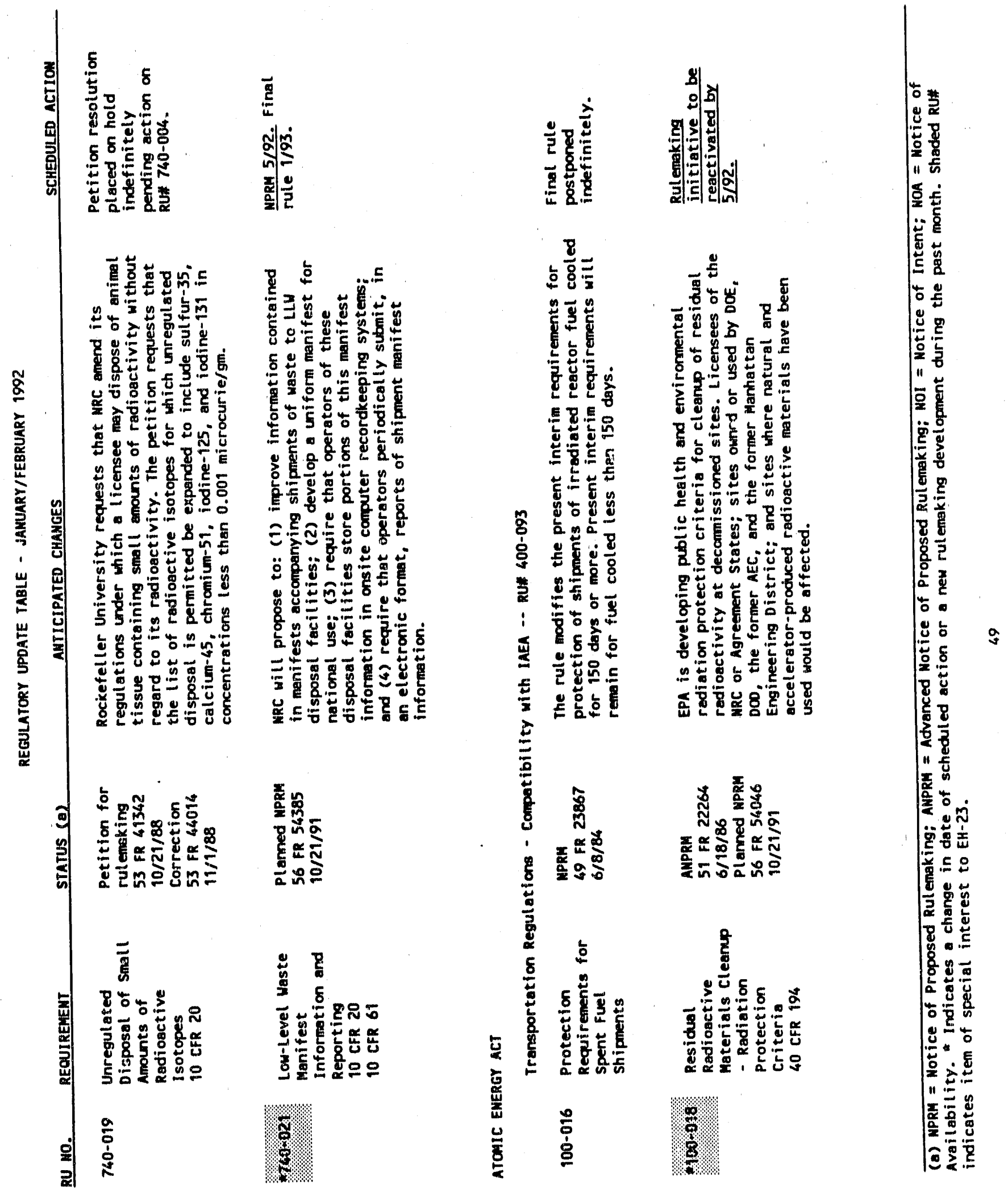




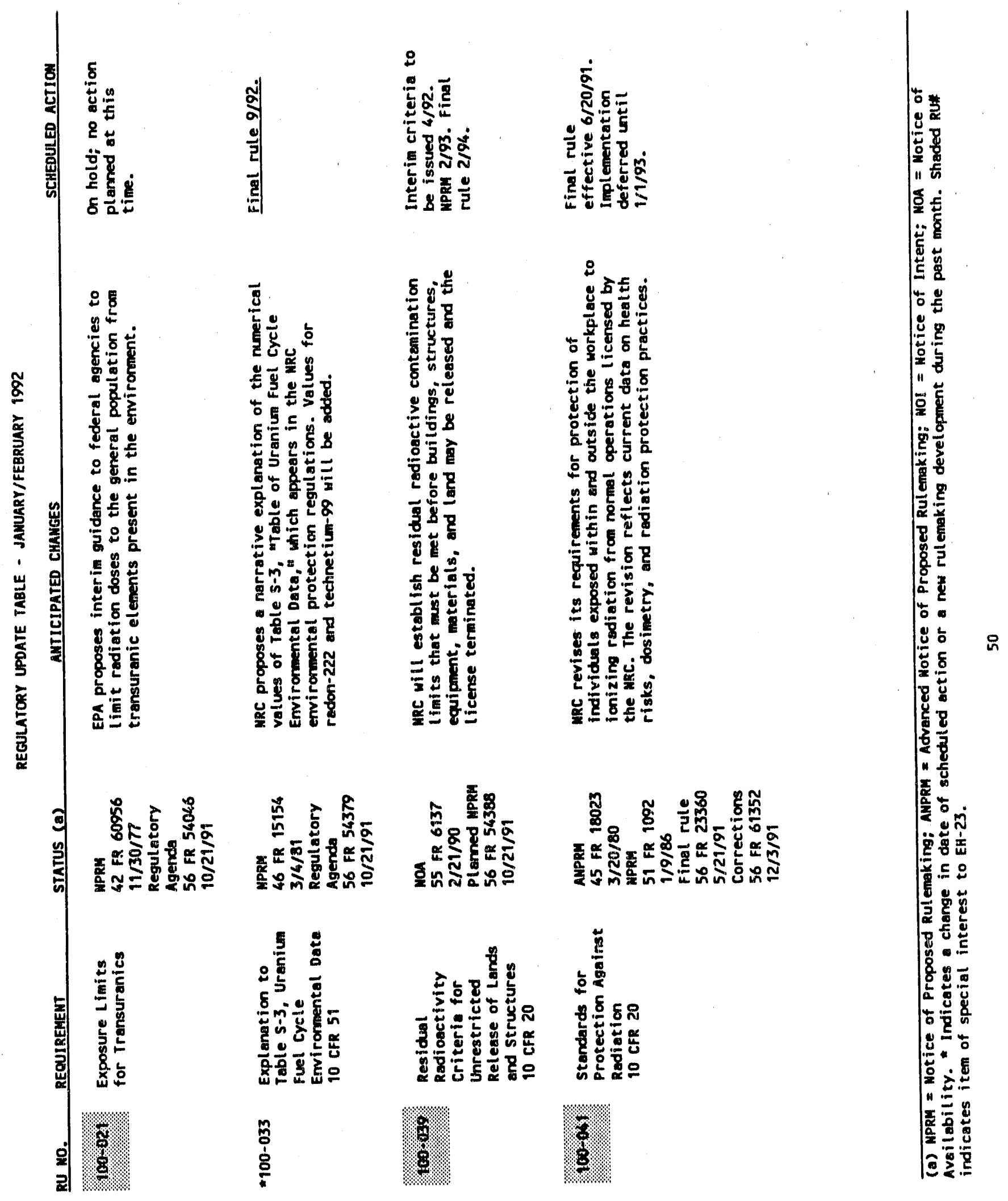




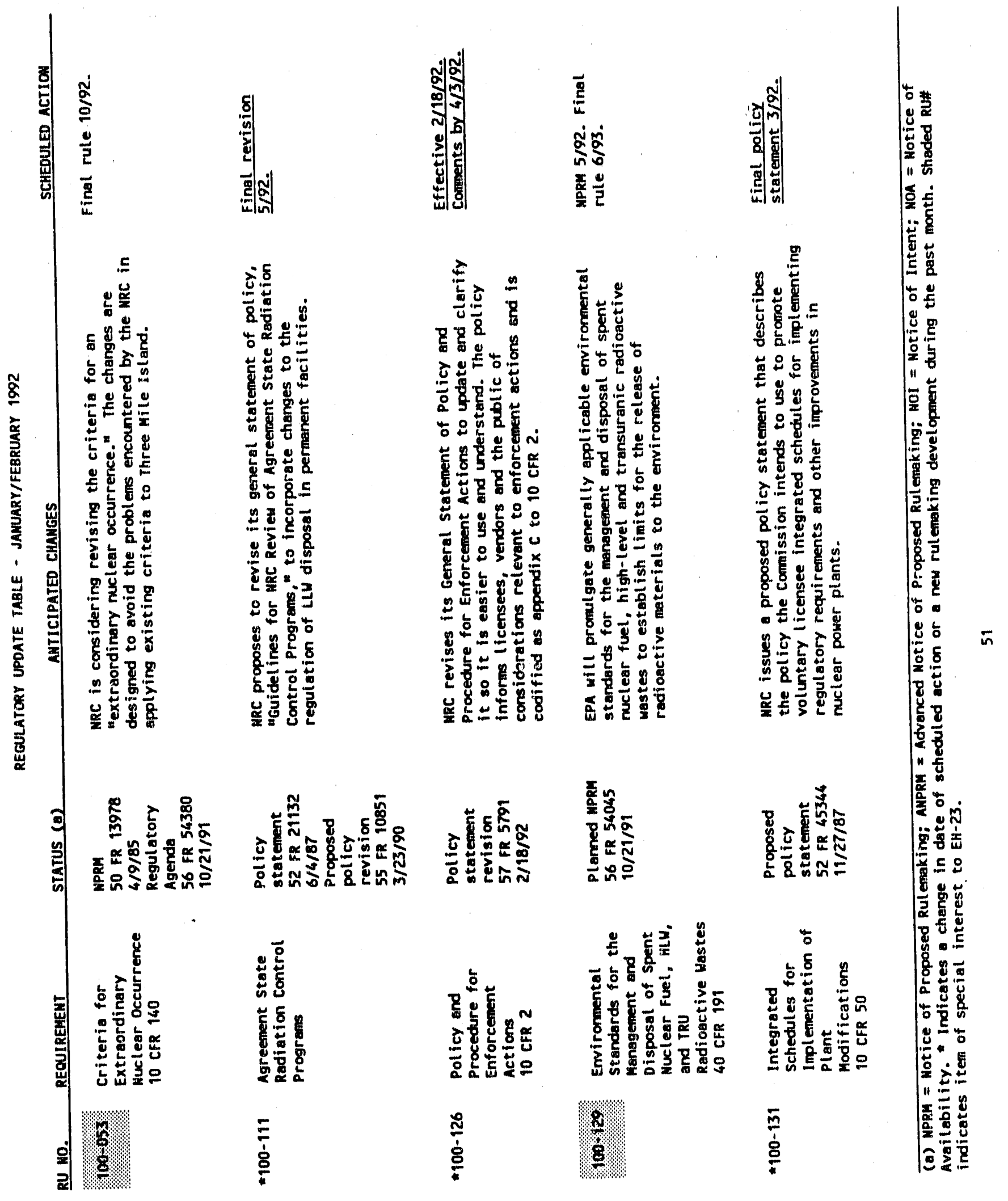



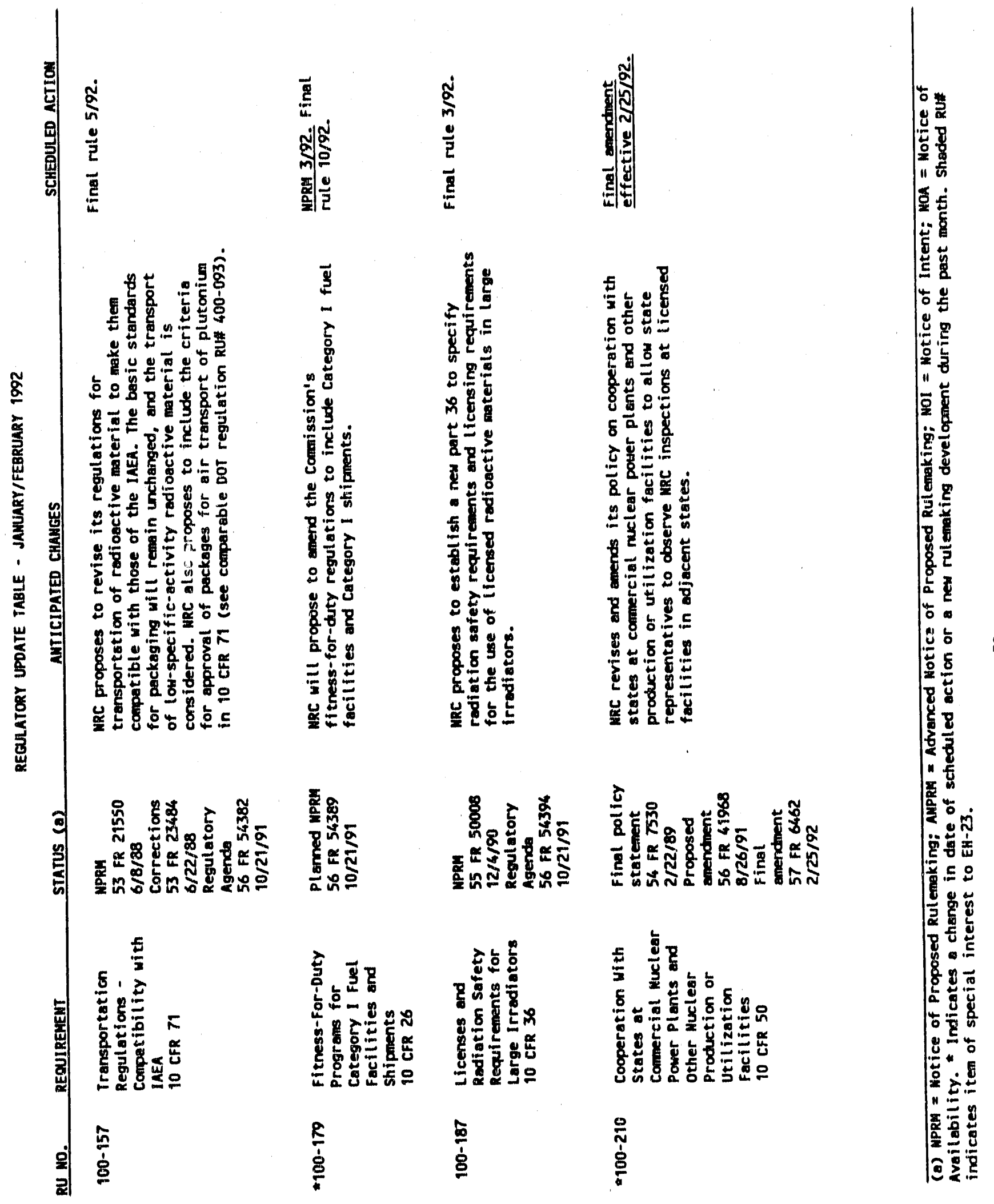

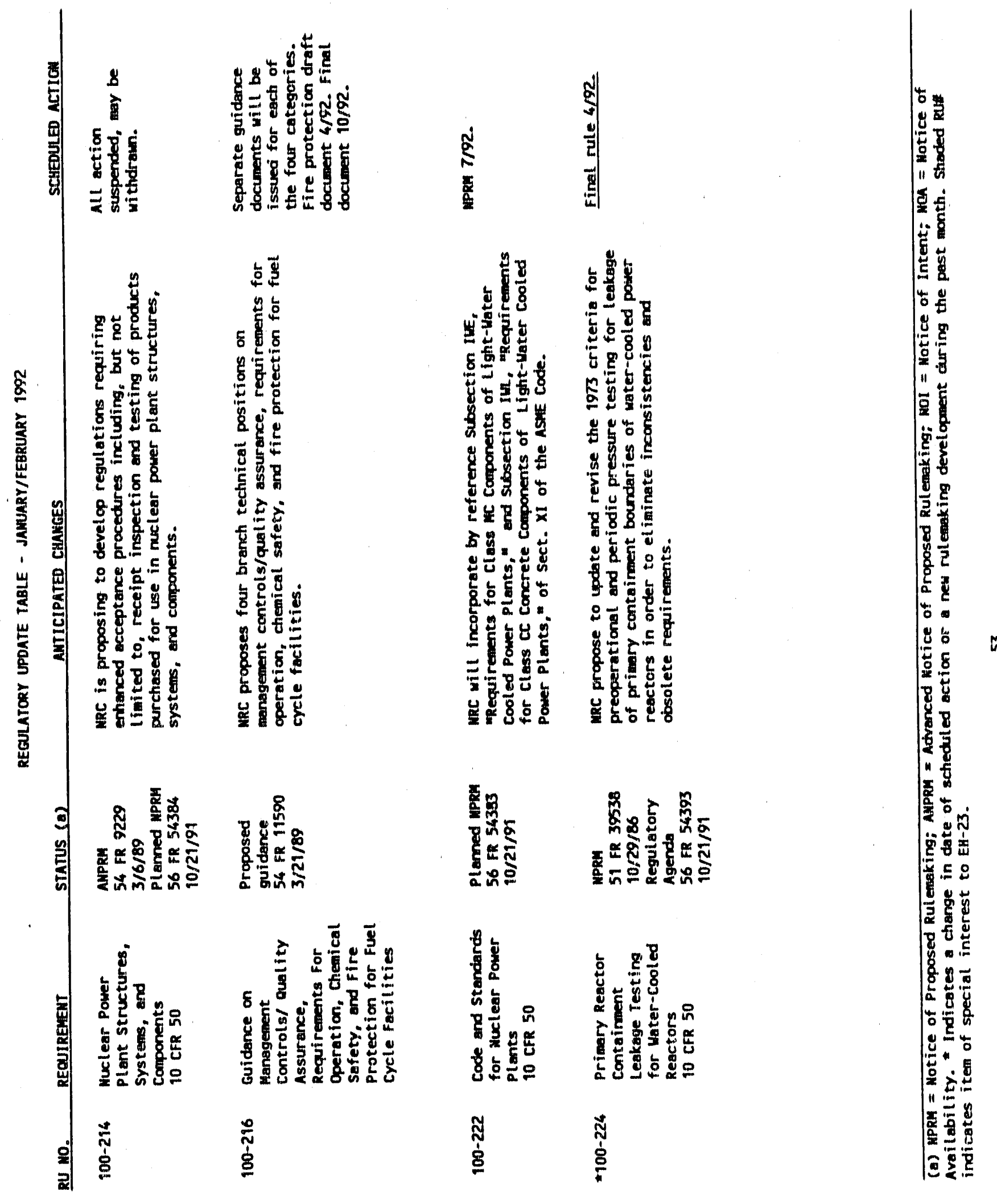


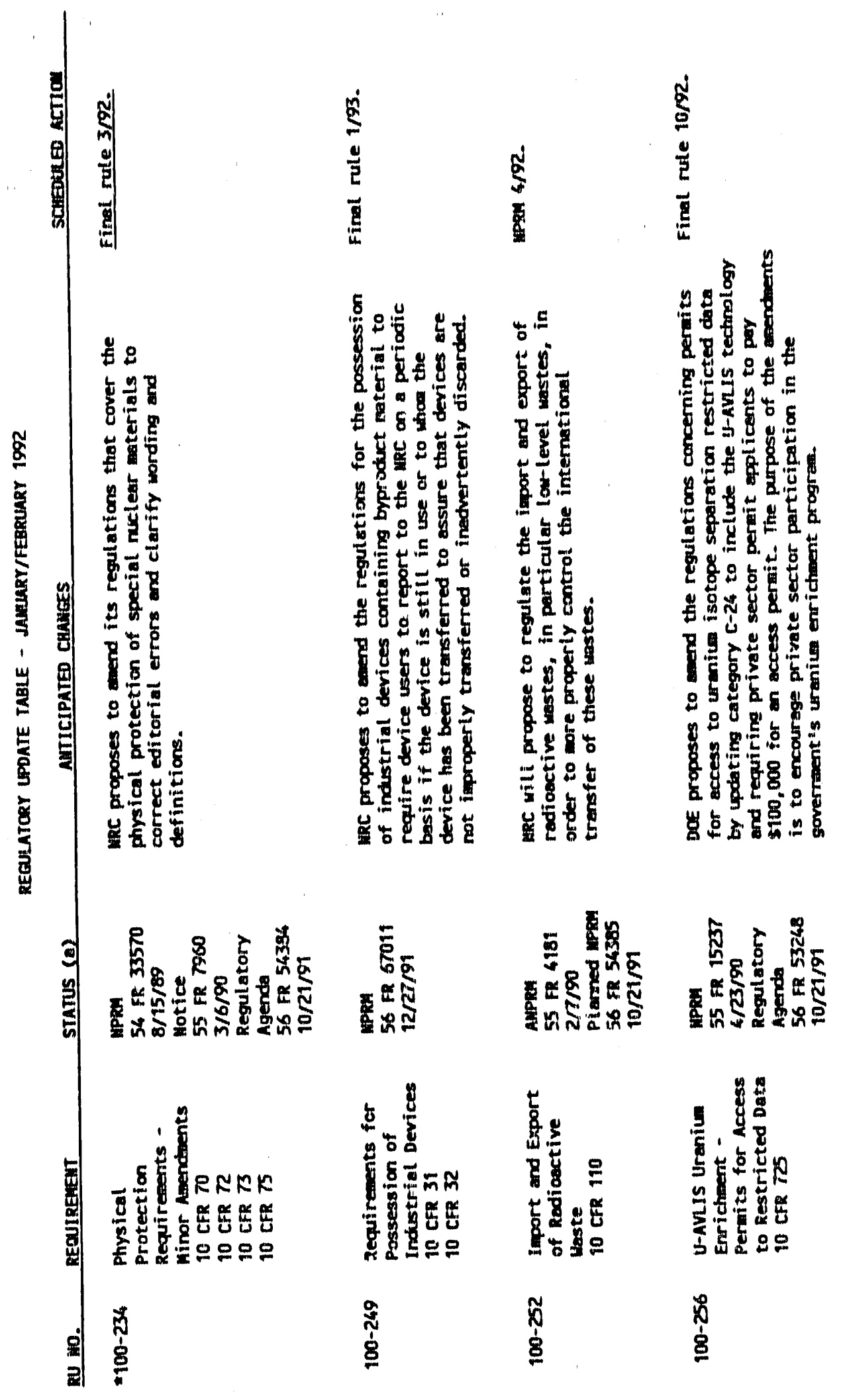




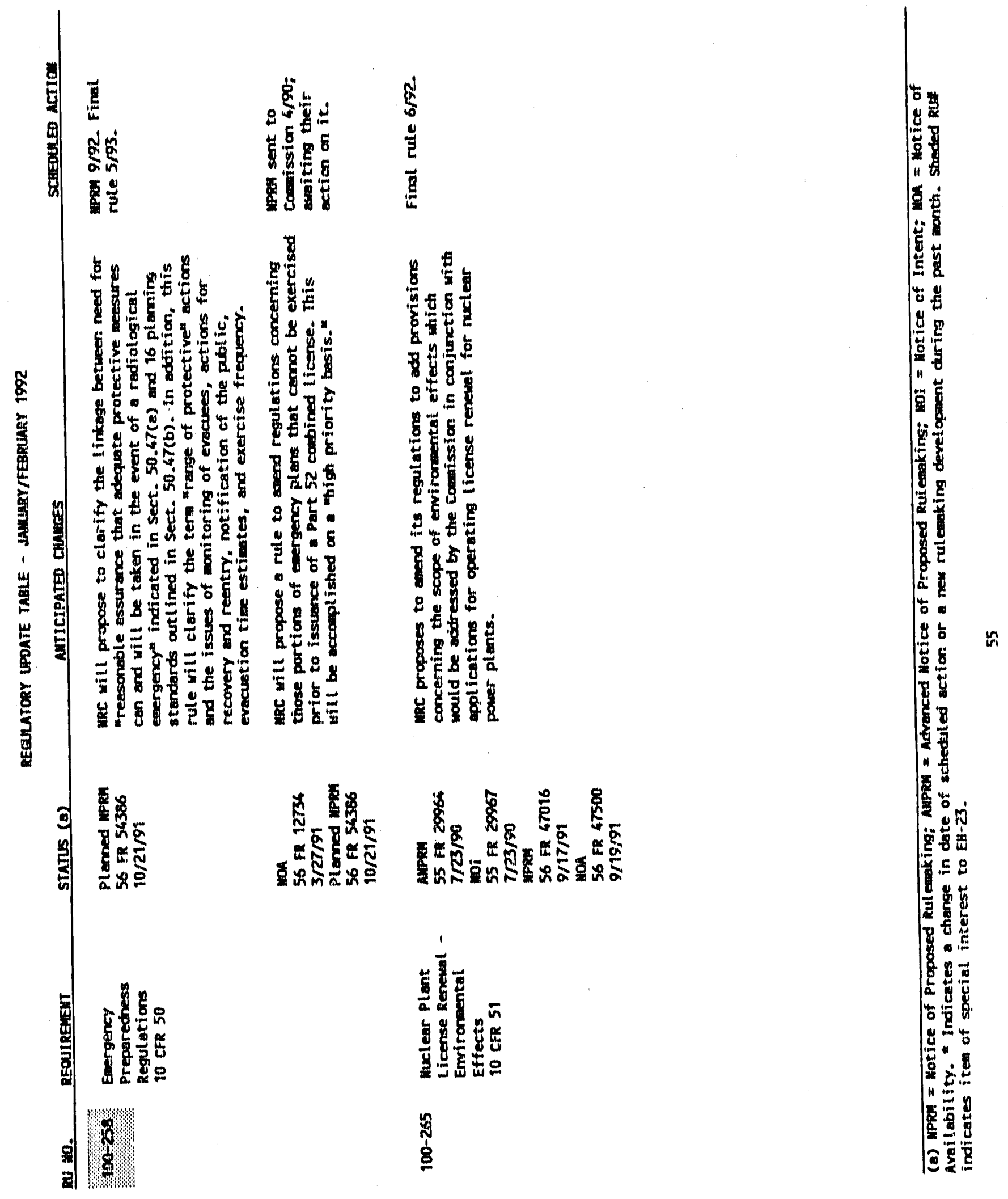




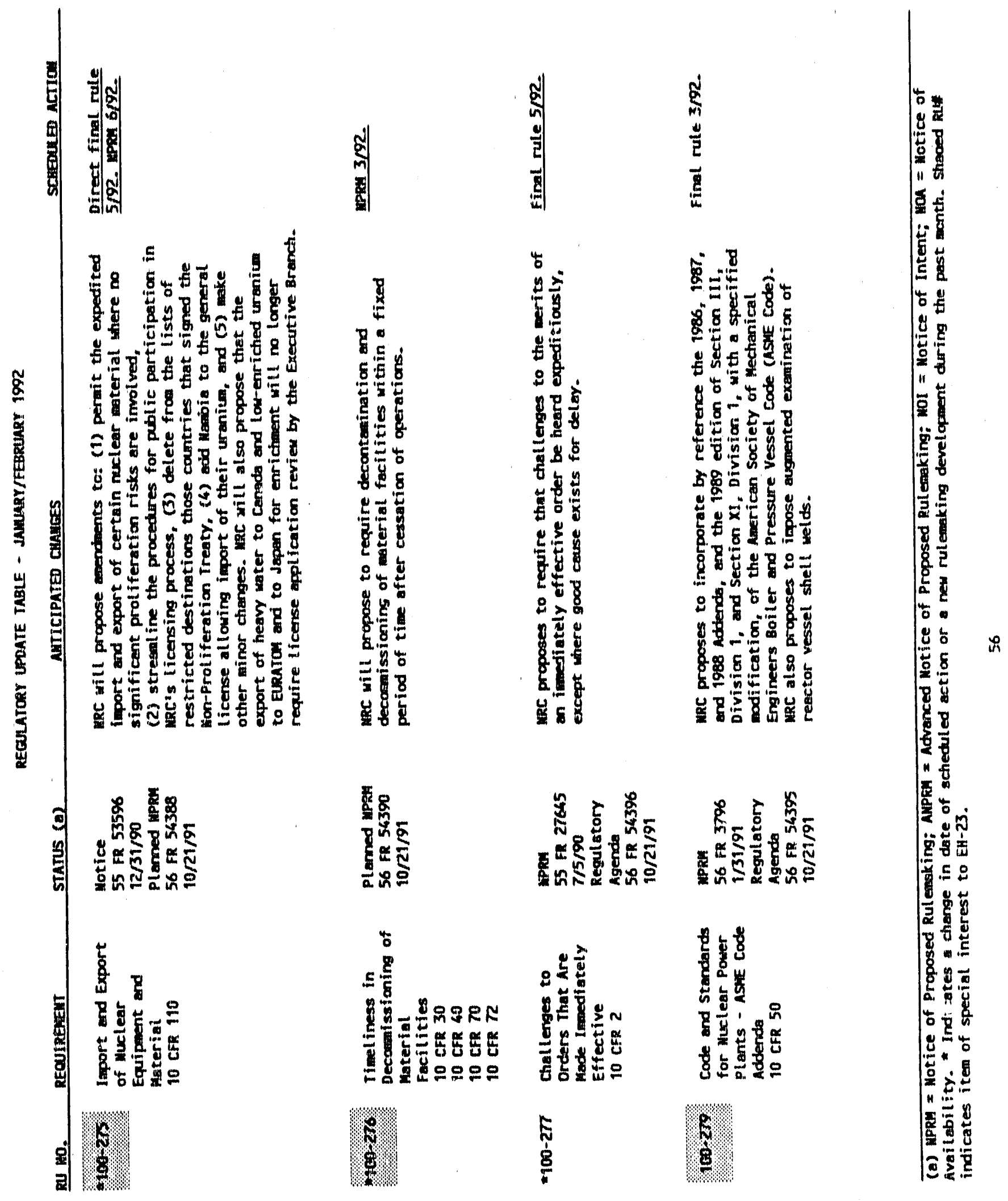




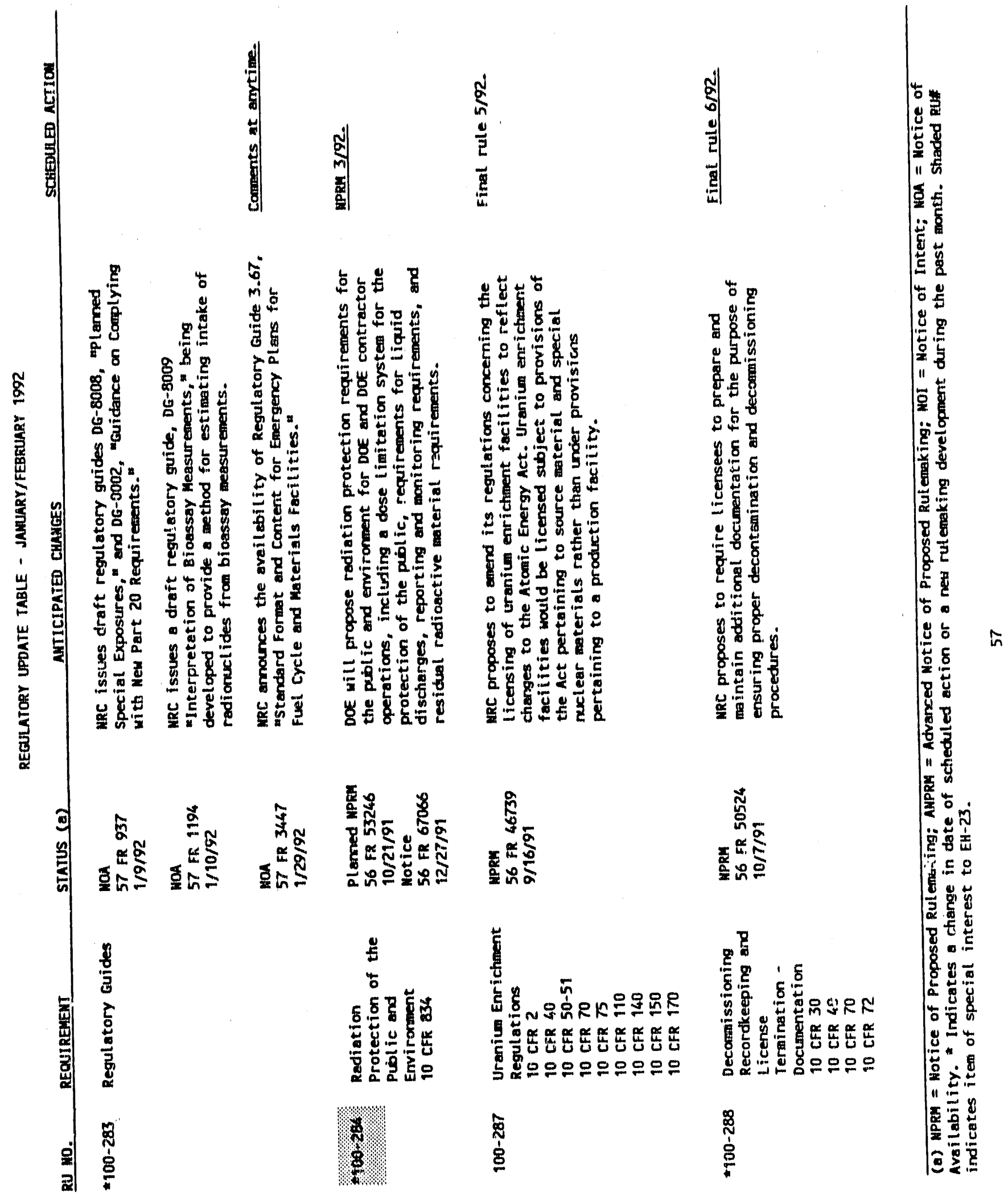




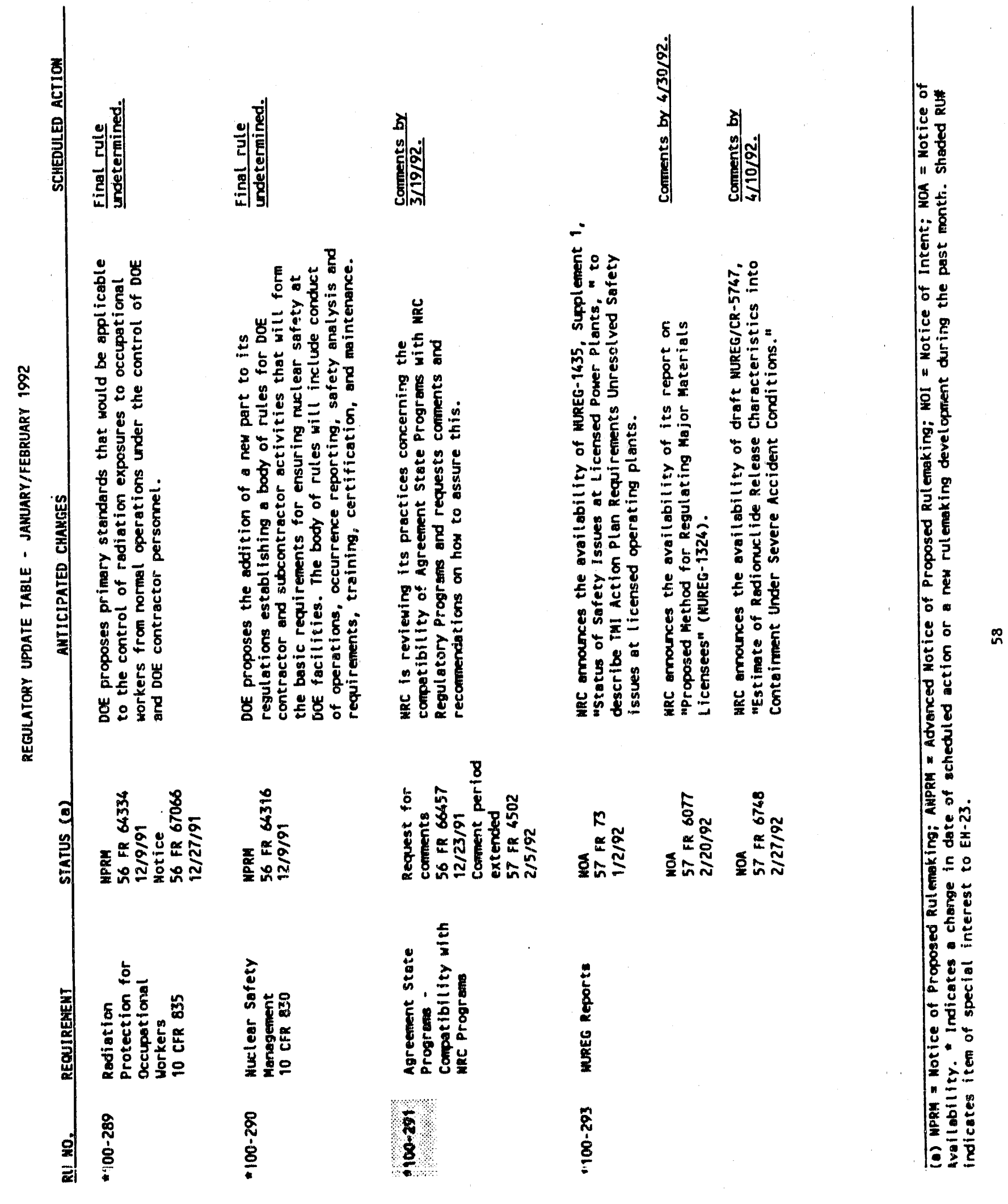




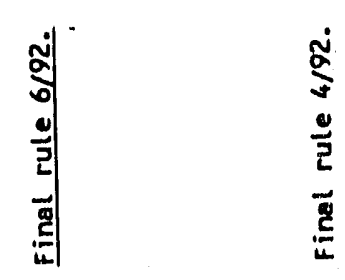

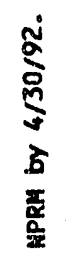
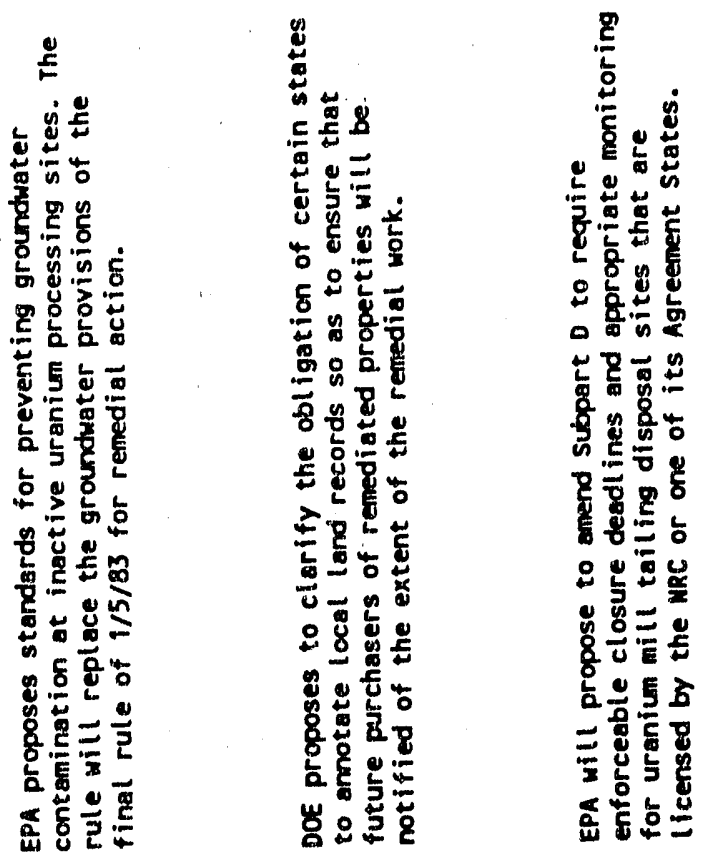

$\underset{\substack{\alpha \\ \frac{\alpha}{2}}}{\frac{\alpha}{2}}$

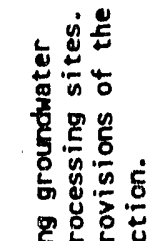

乐崖

造造言。

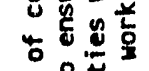

客要

苟告

尊运造

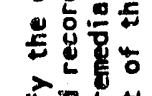

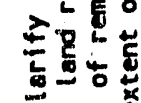

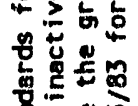

可范

요월

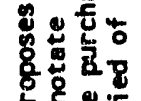

岩造

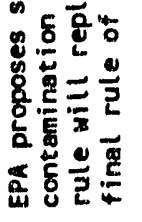
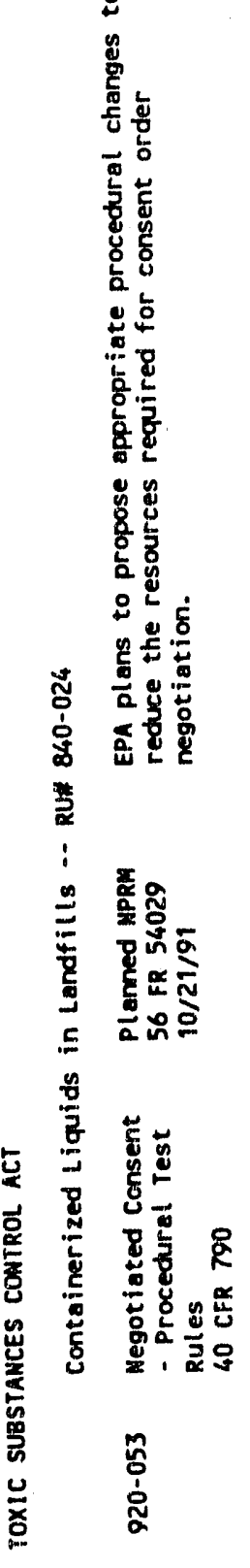

늠

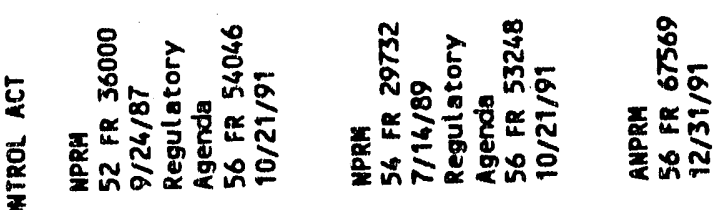

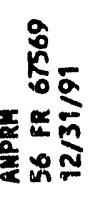

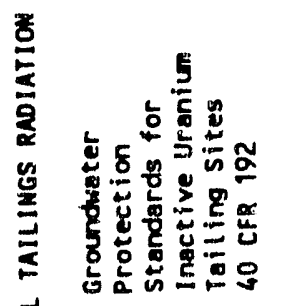

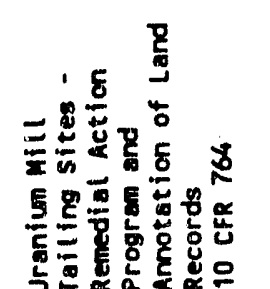

等

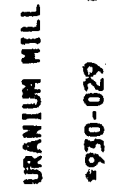

苍

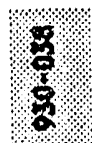



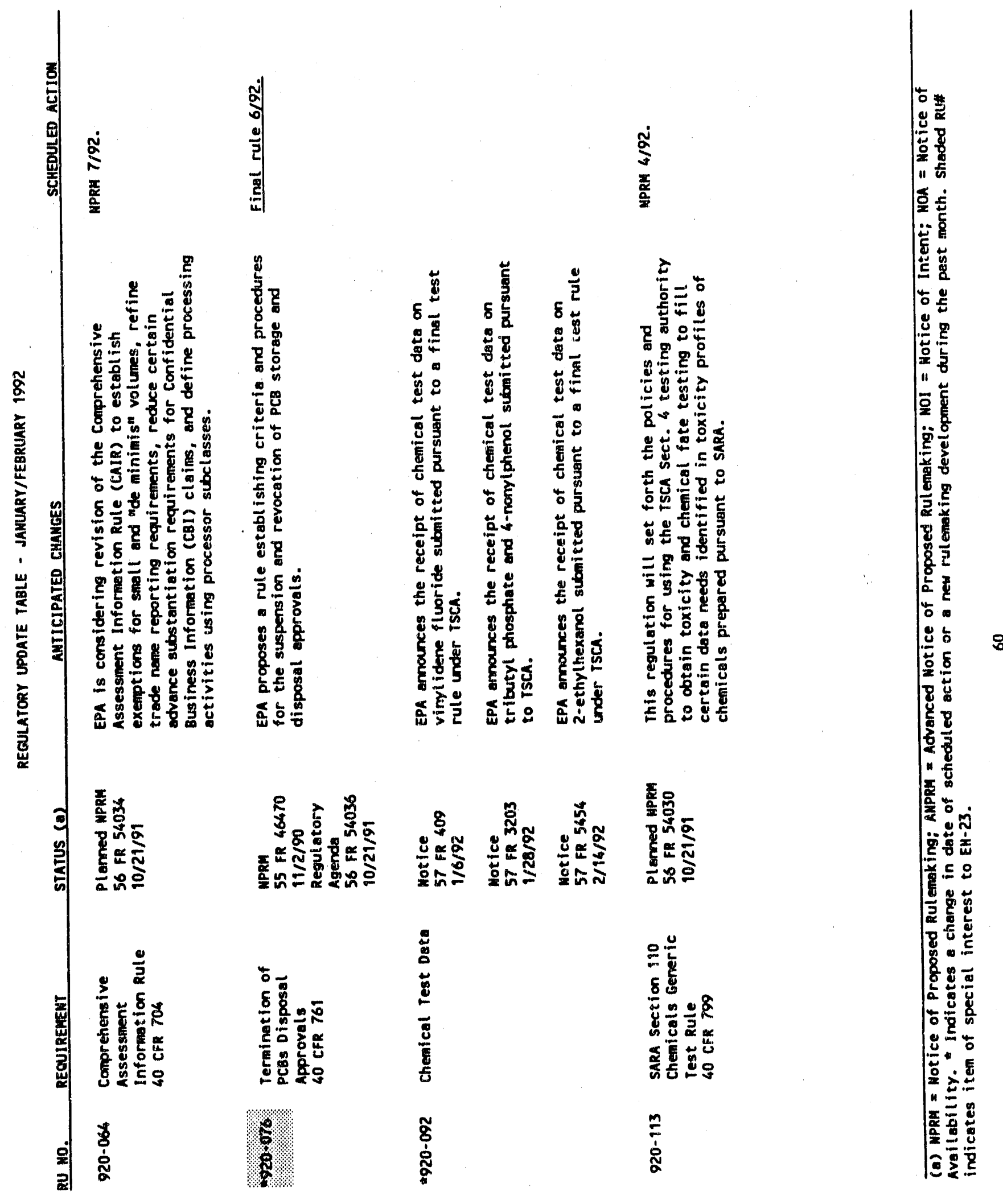


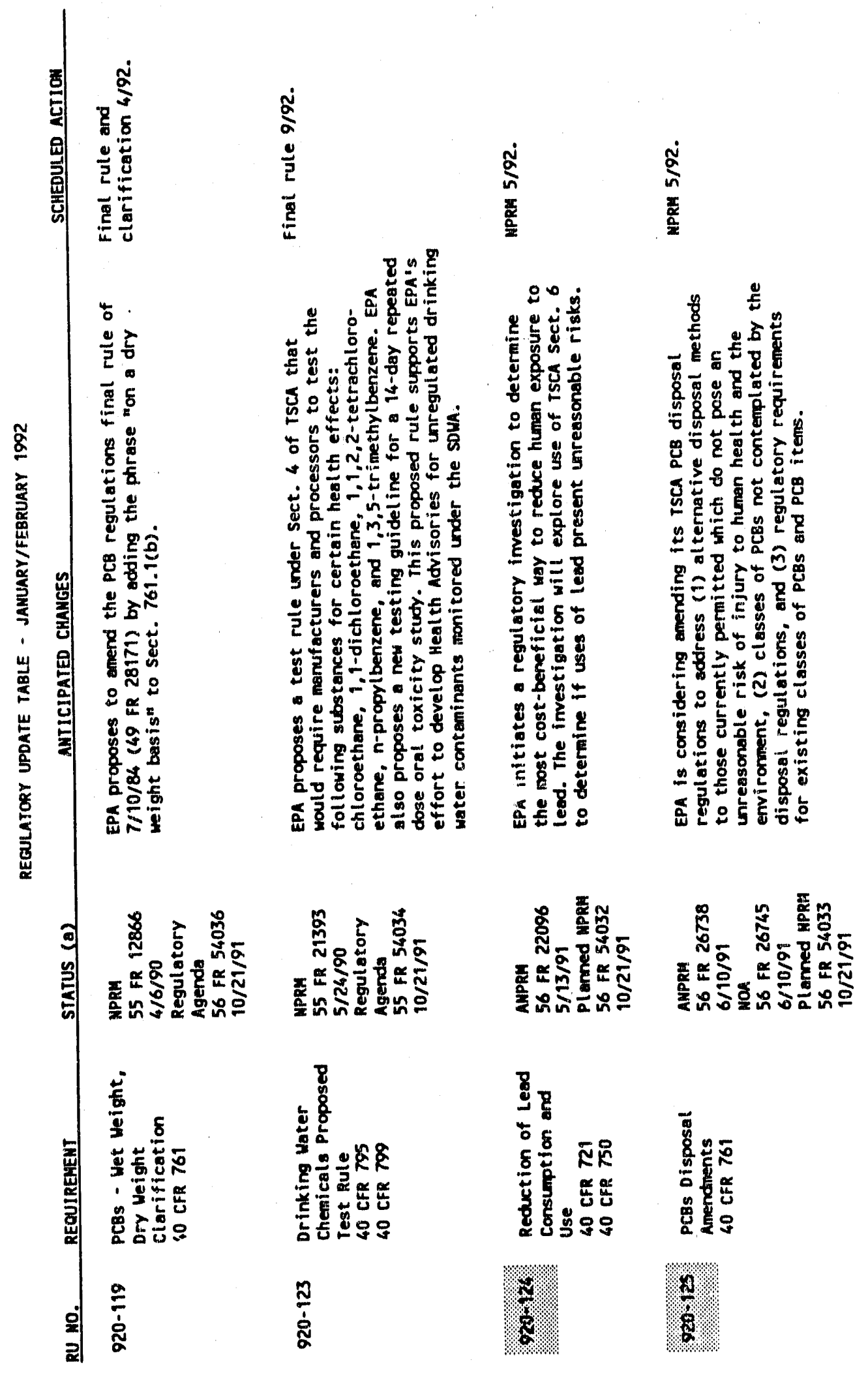




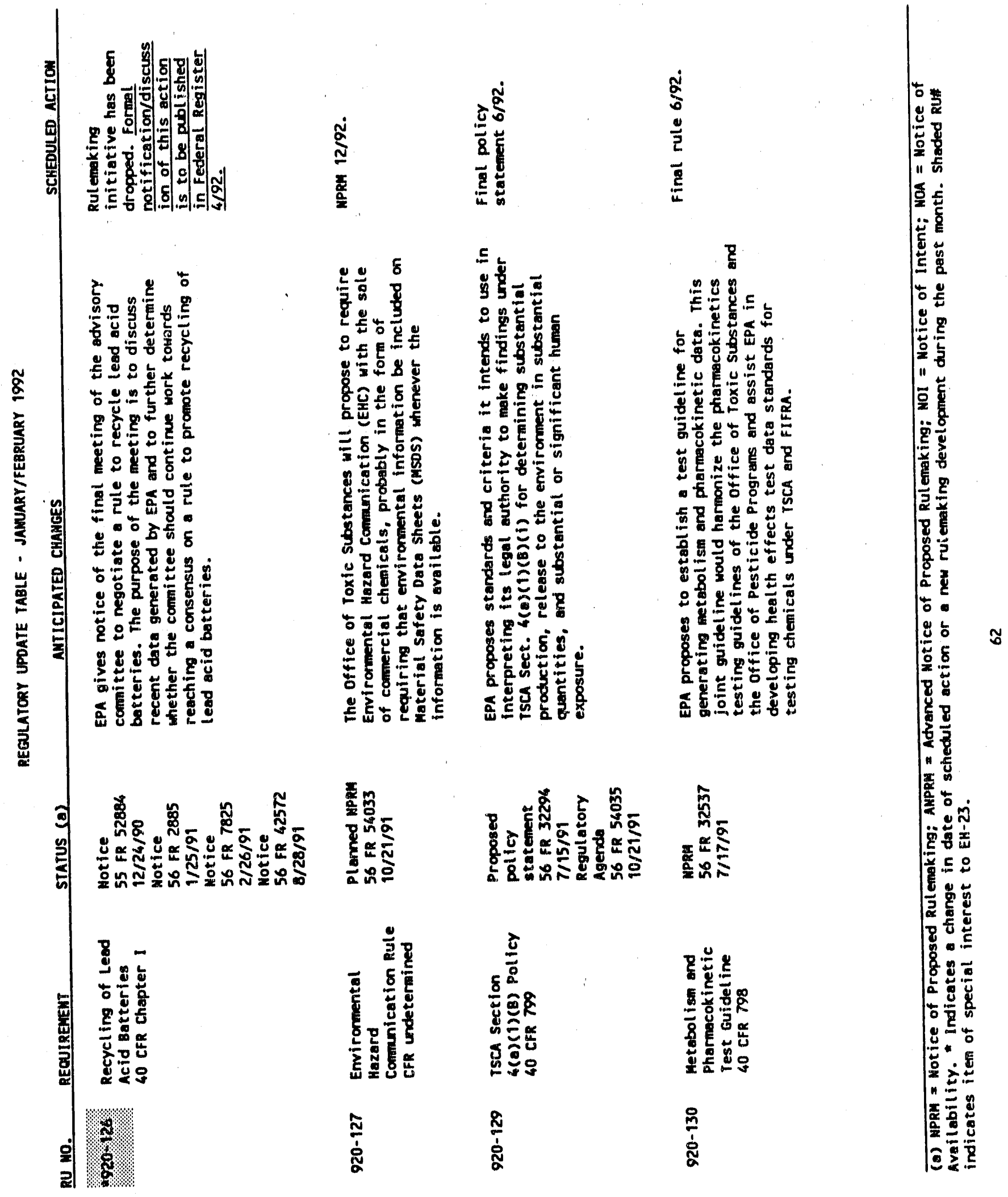




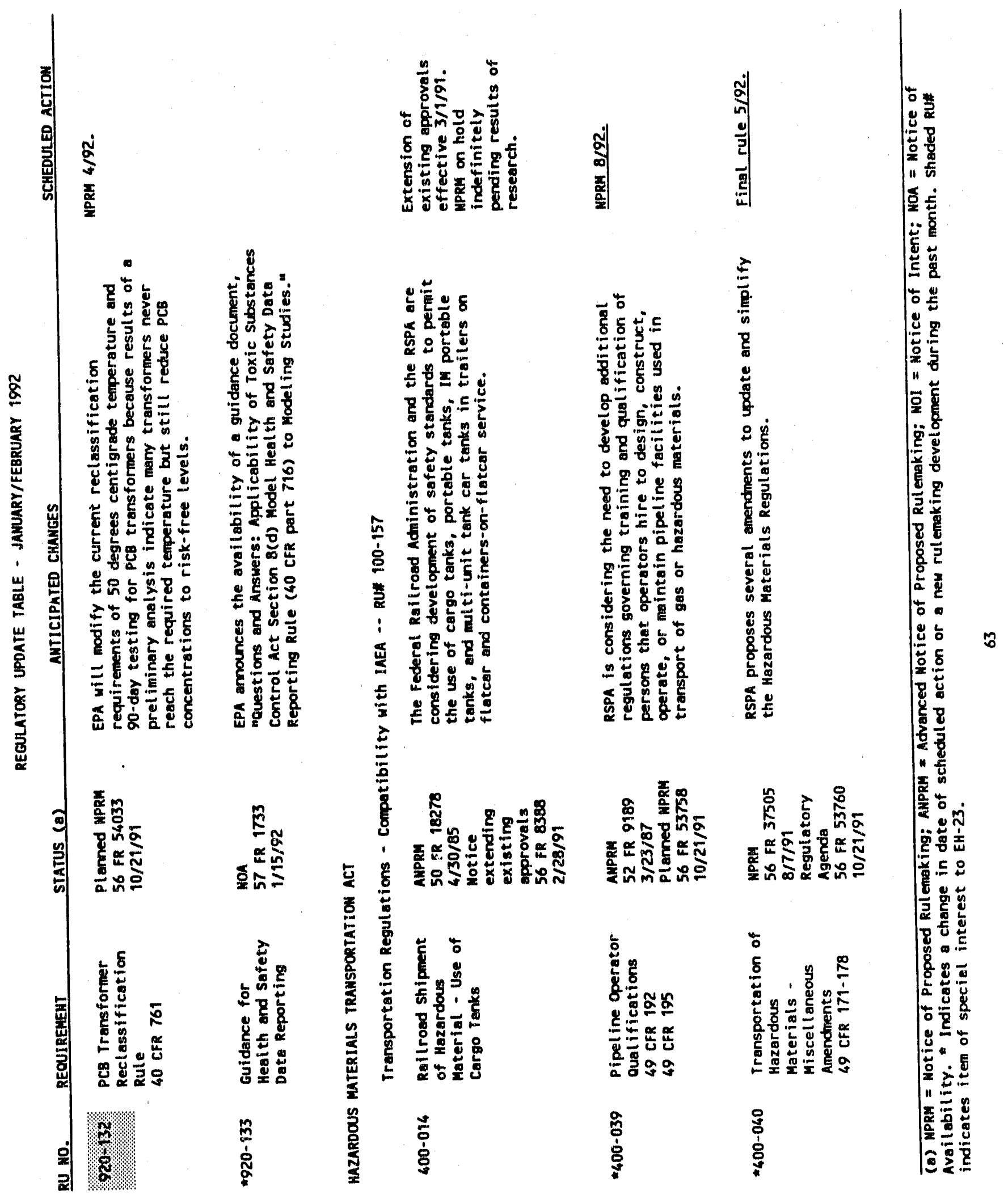




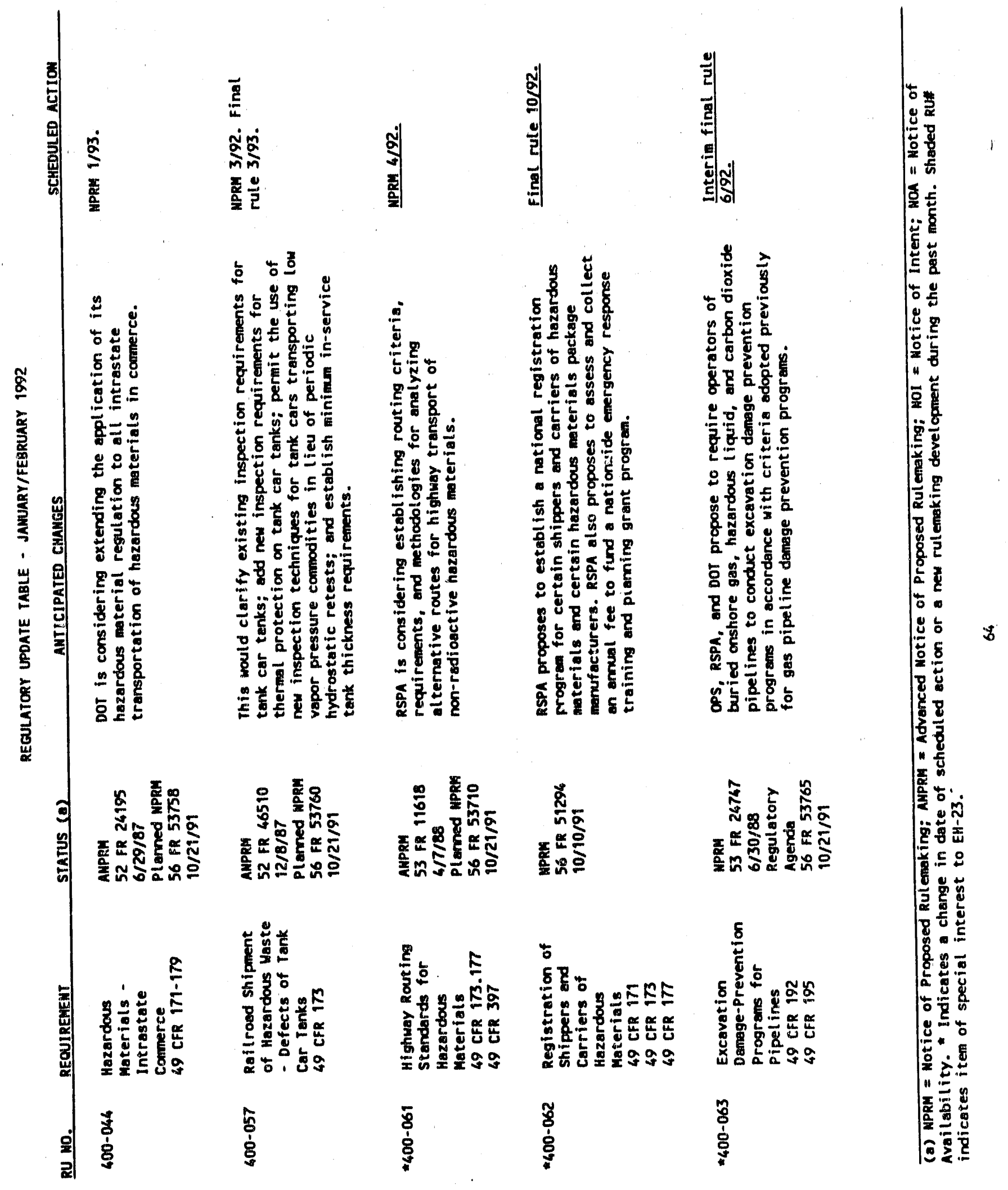




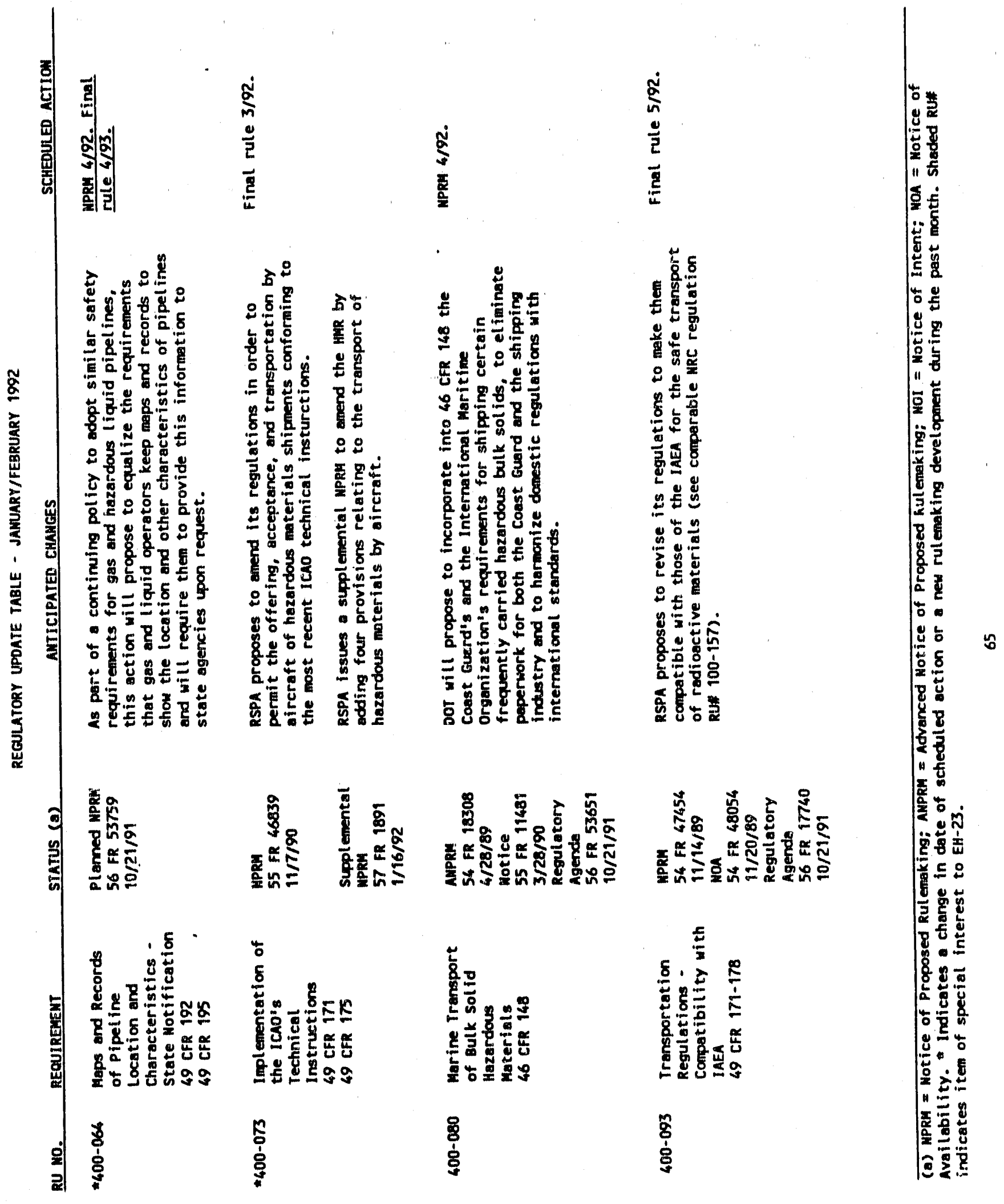




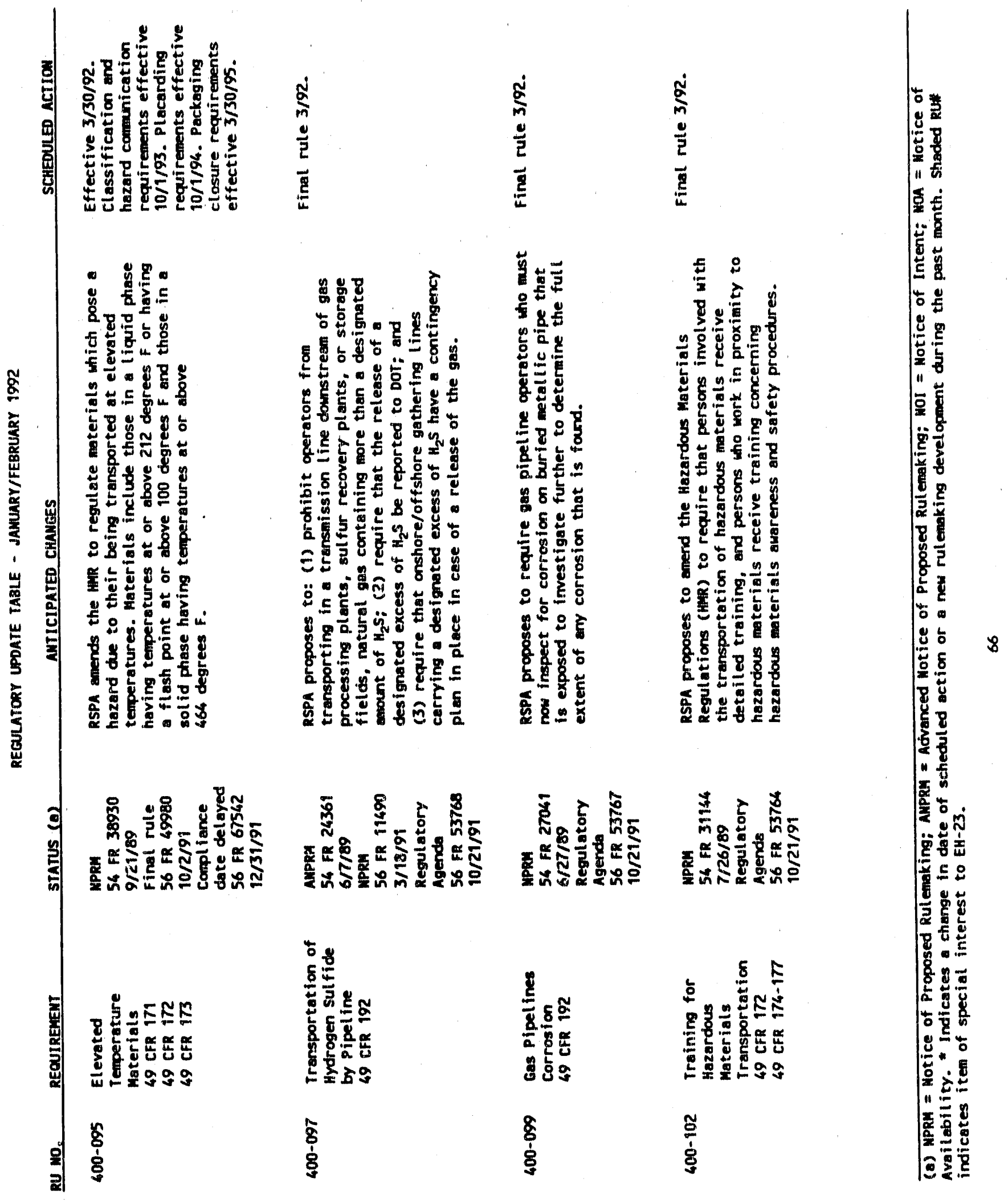




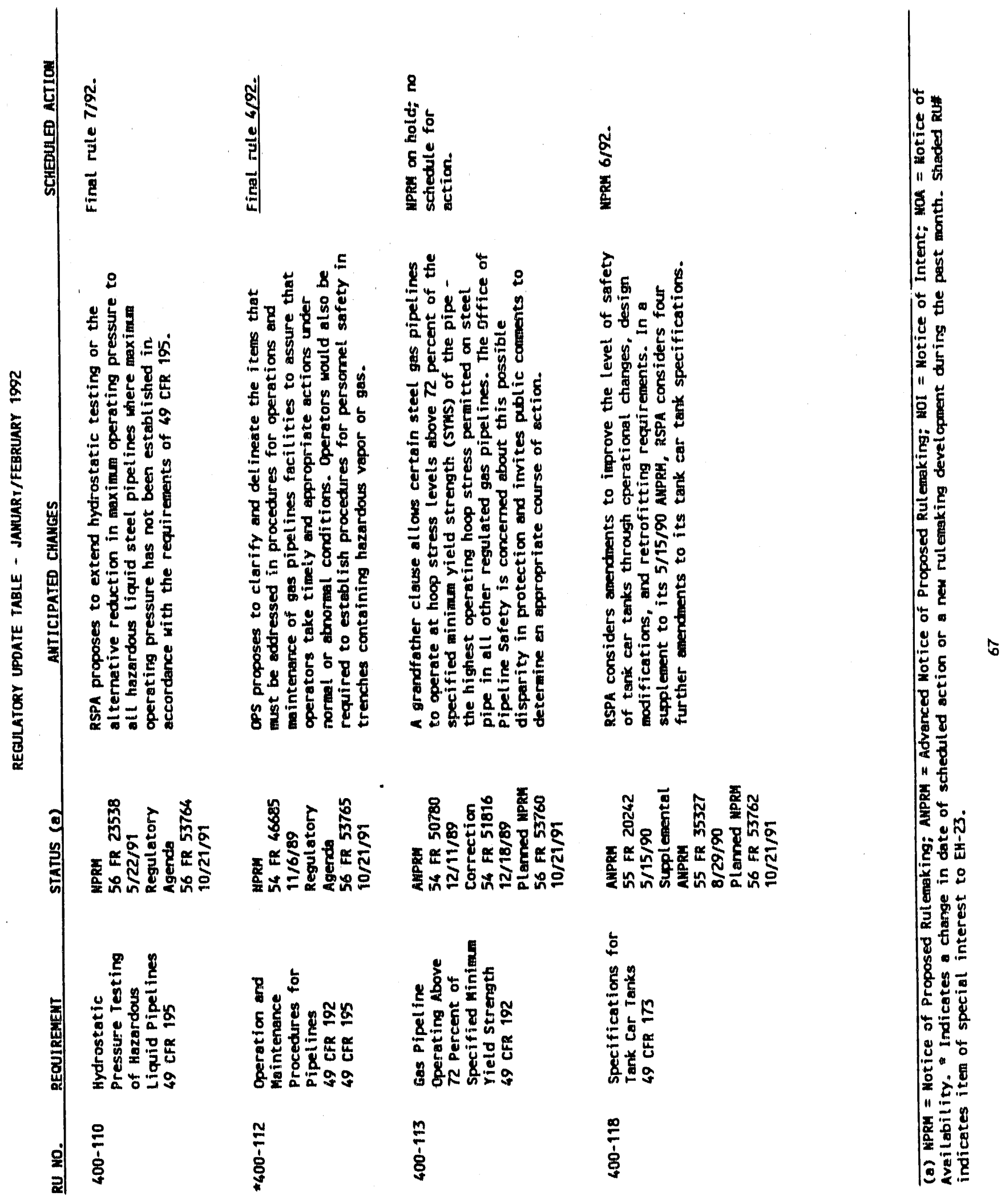



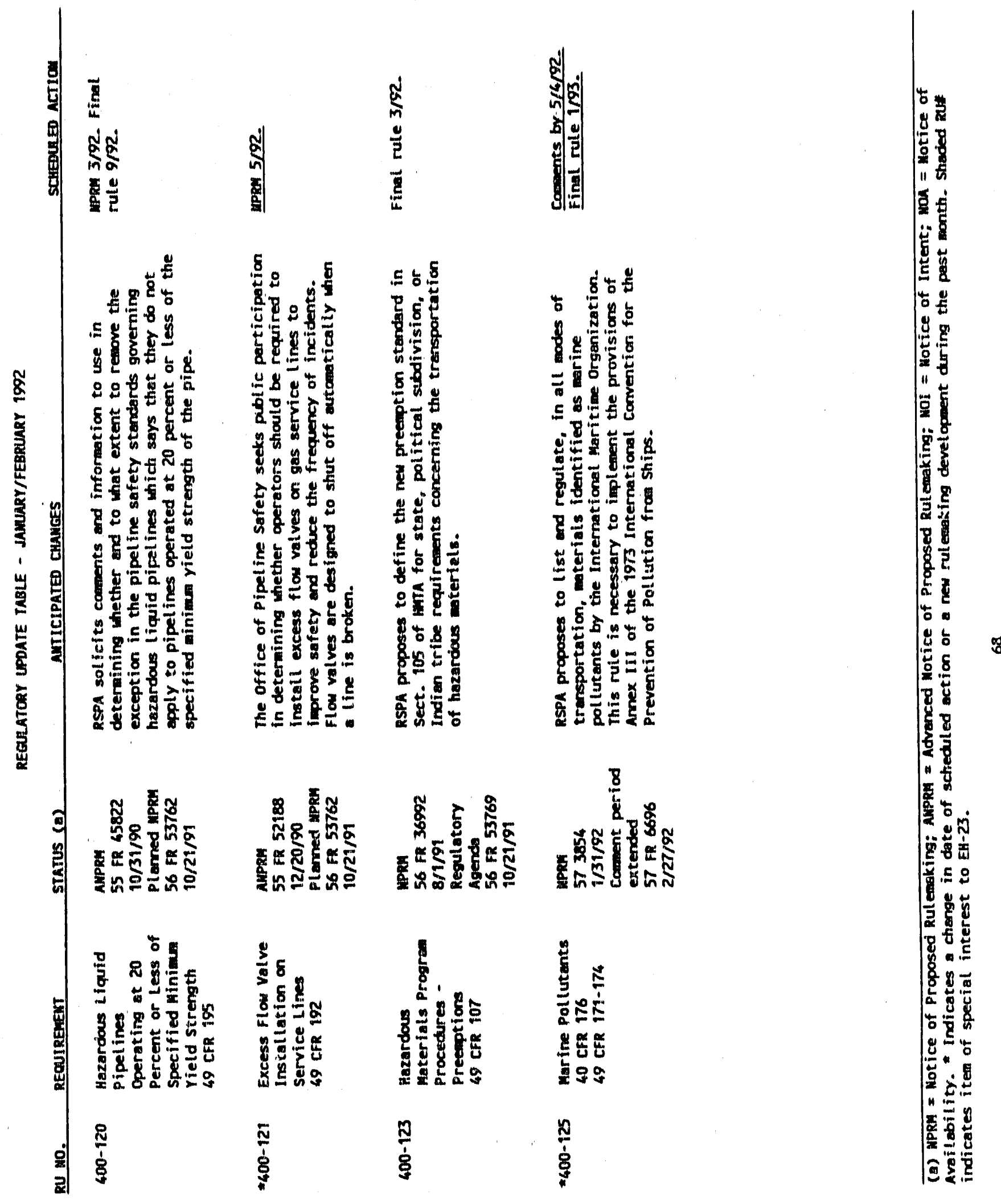

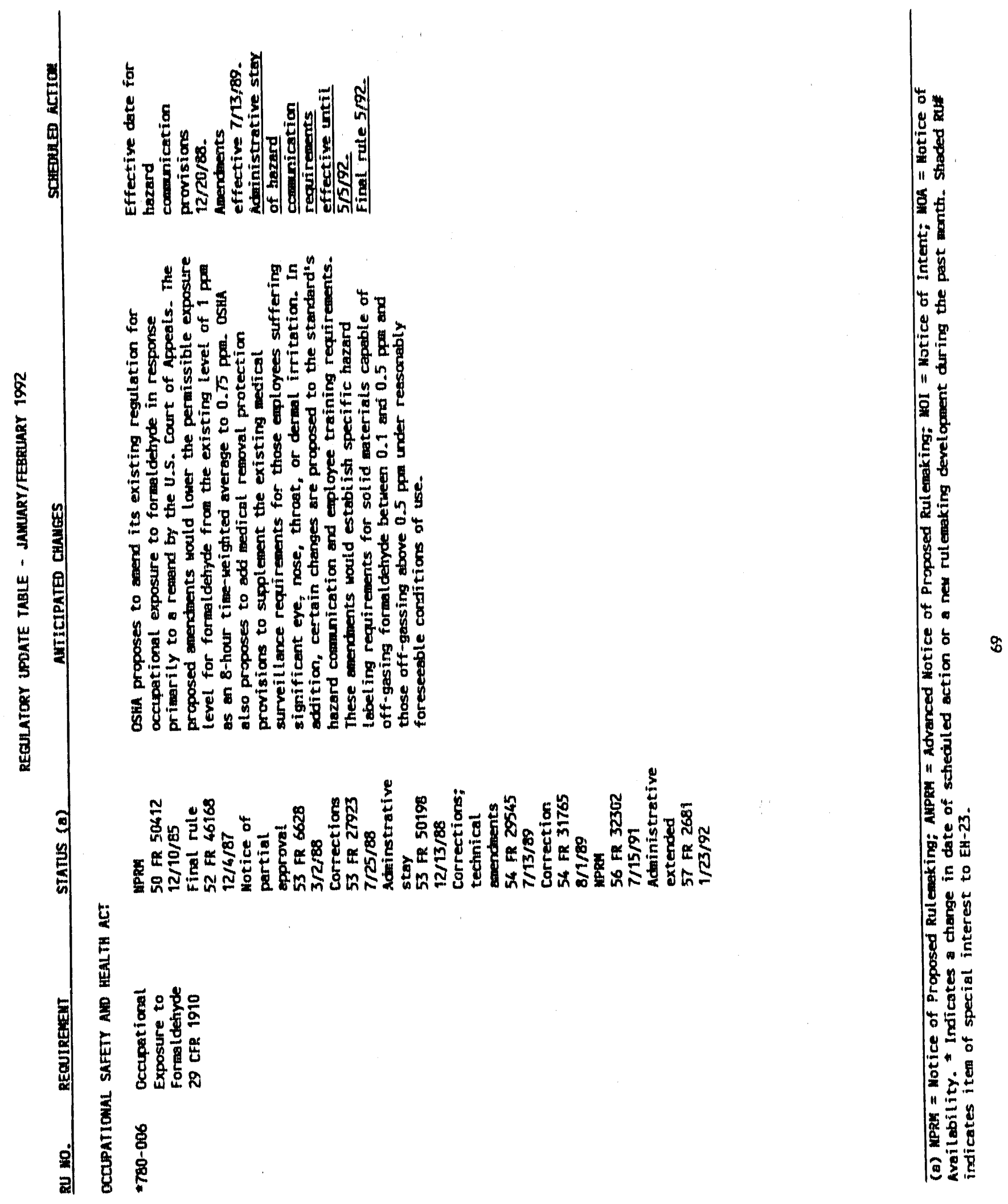

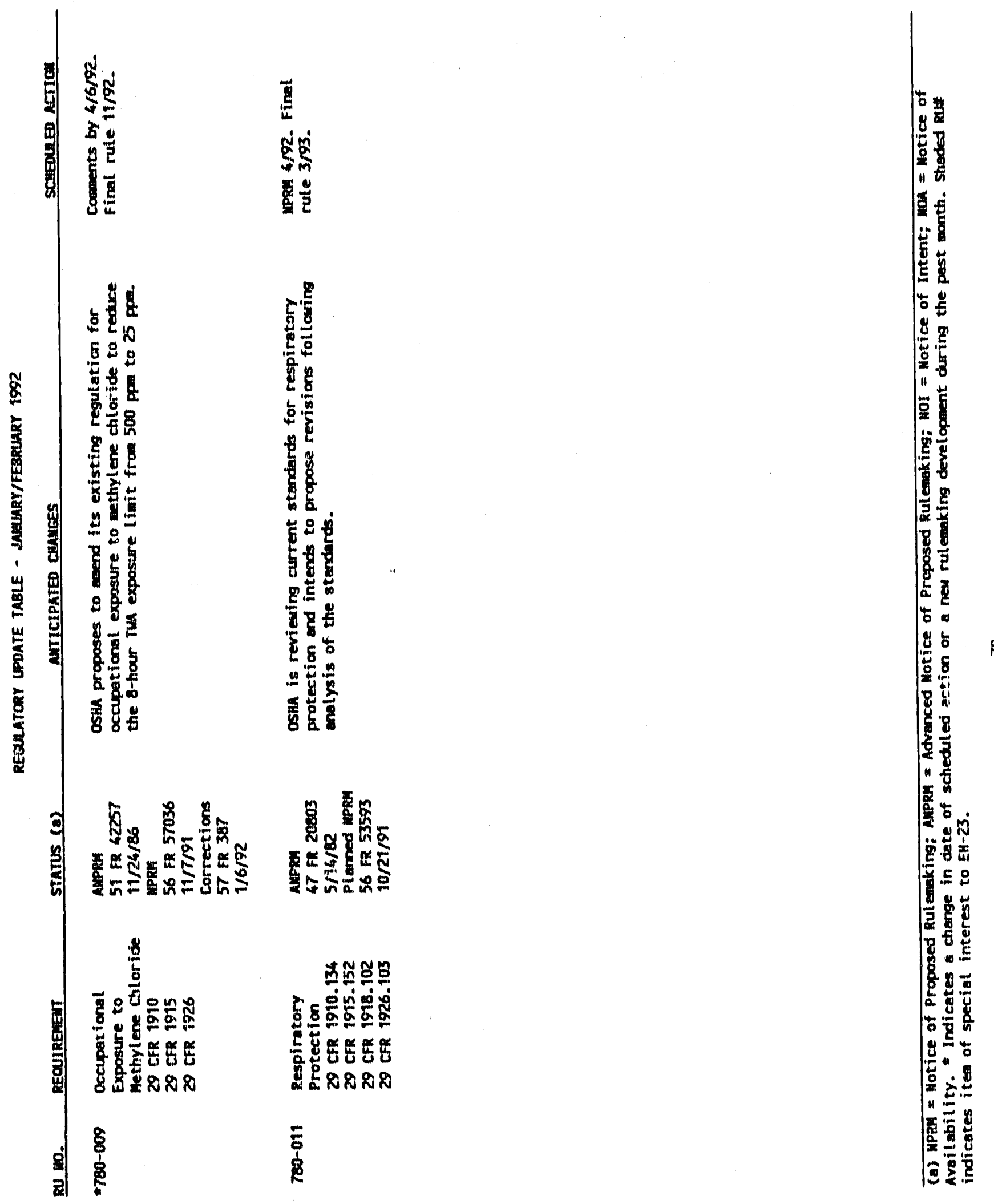

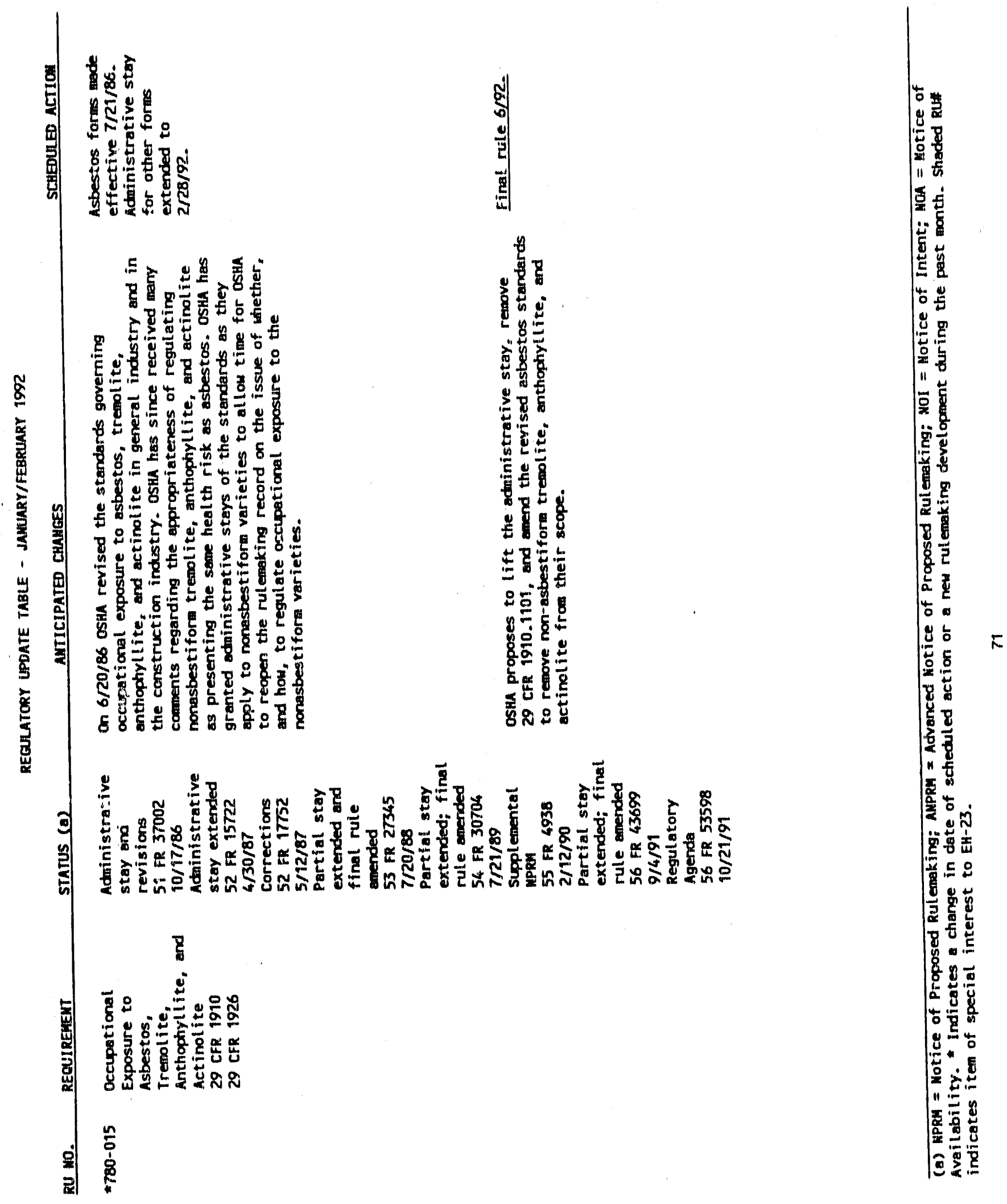


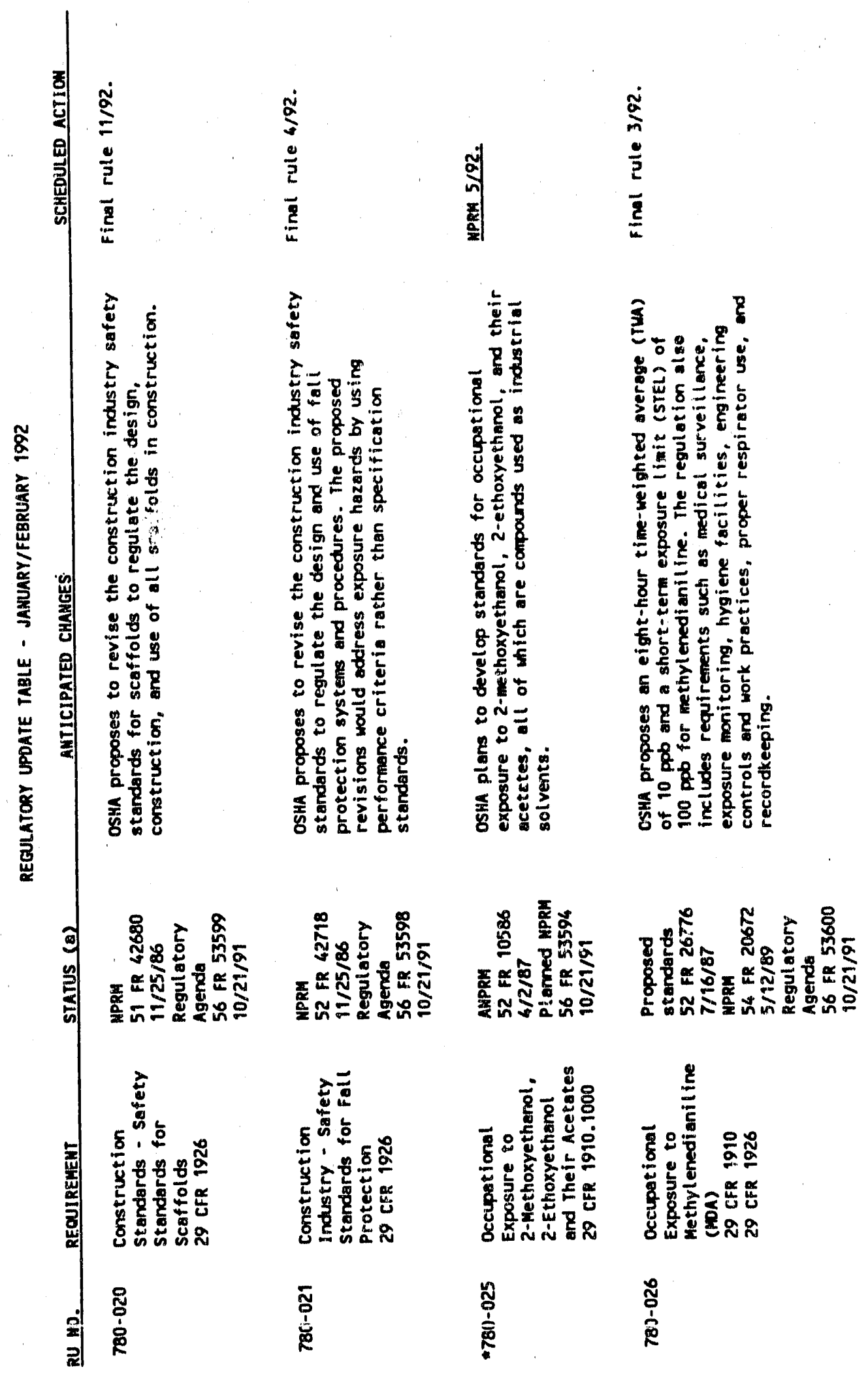




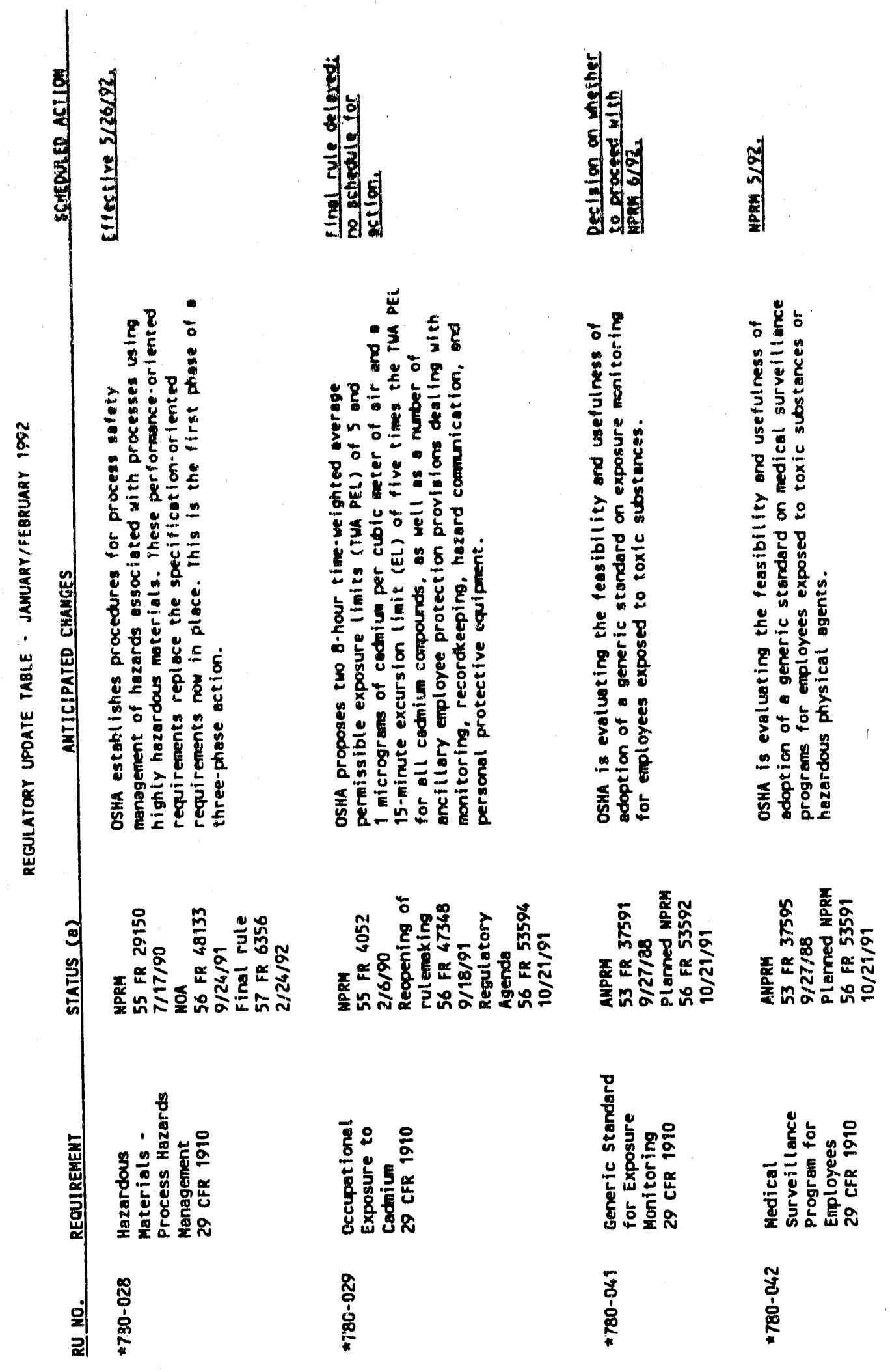




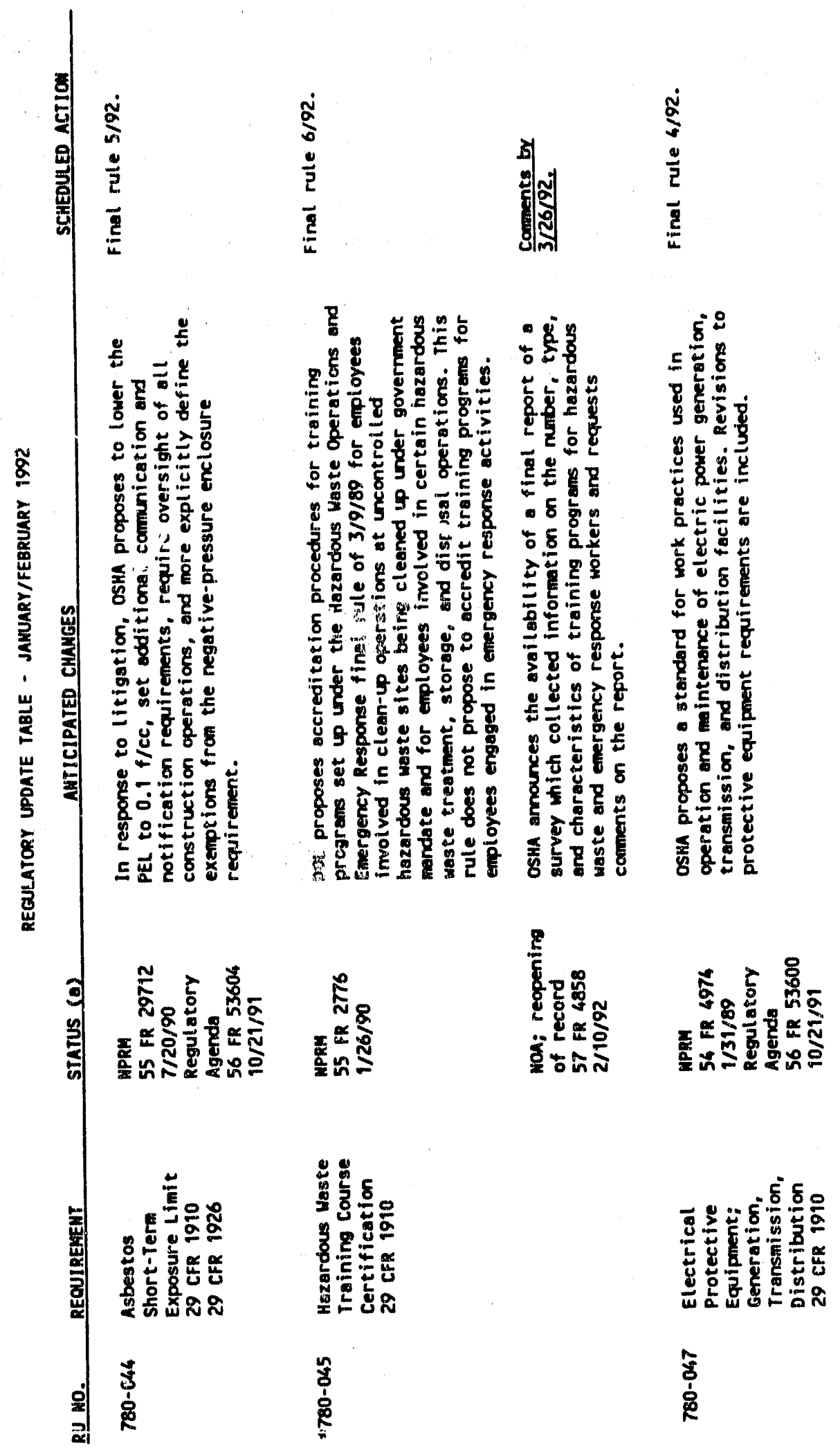




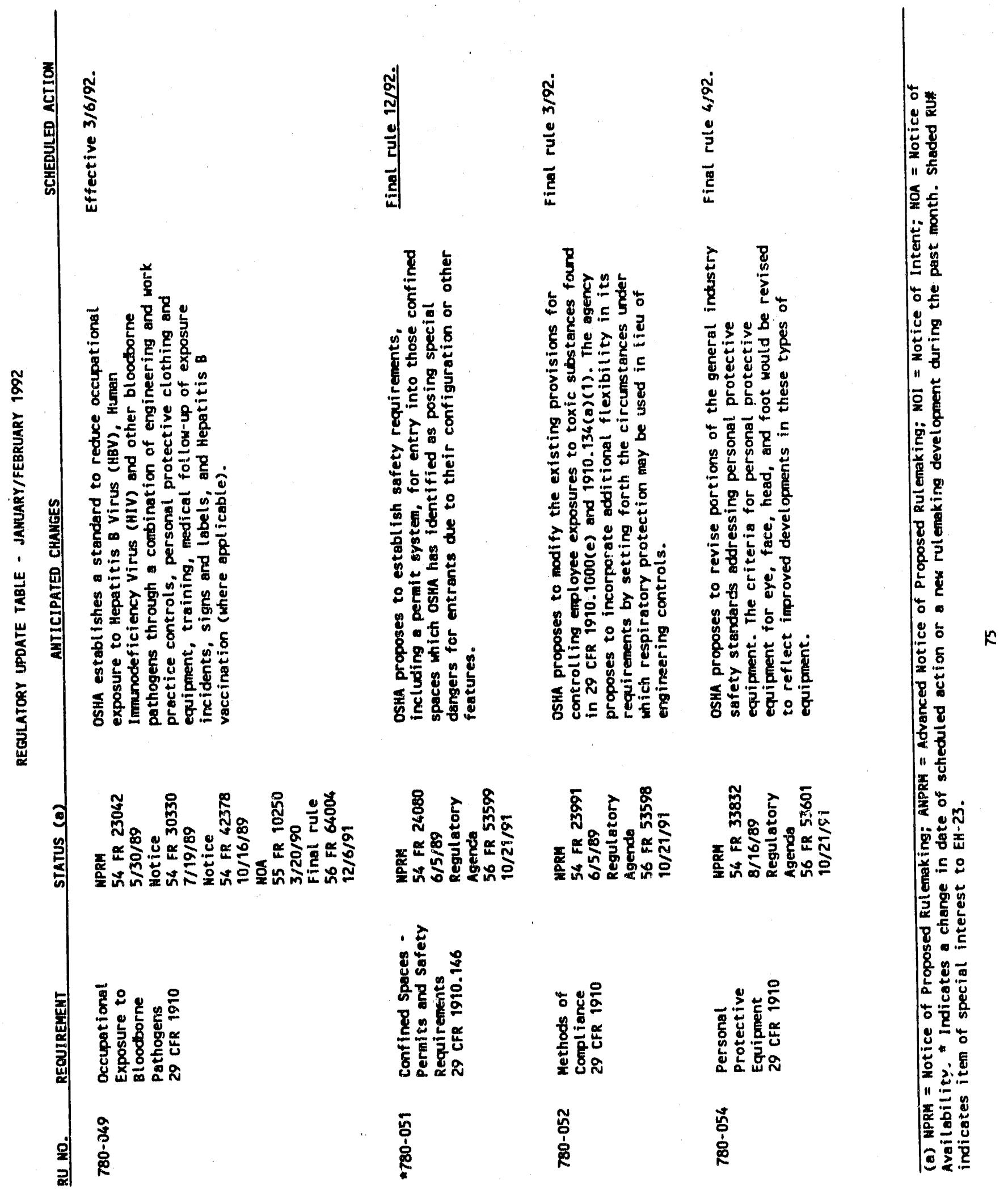




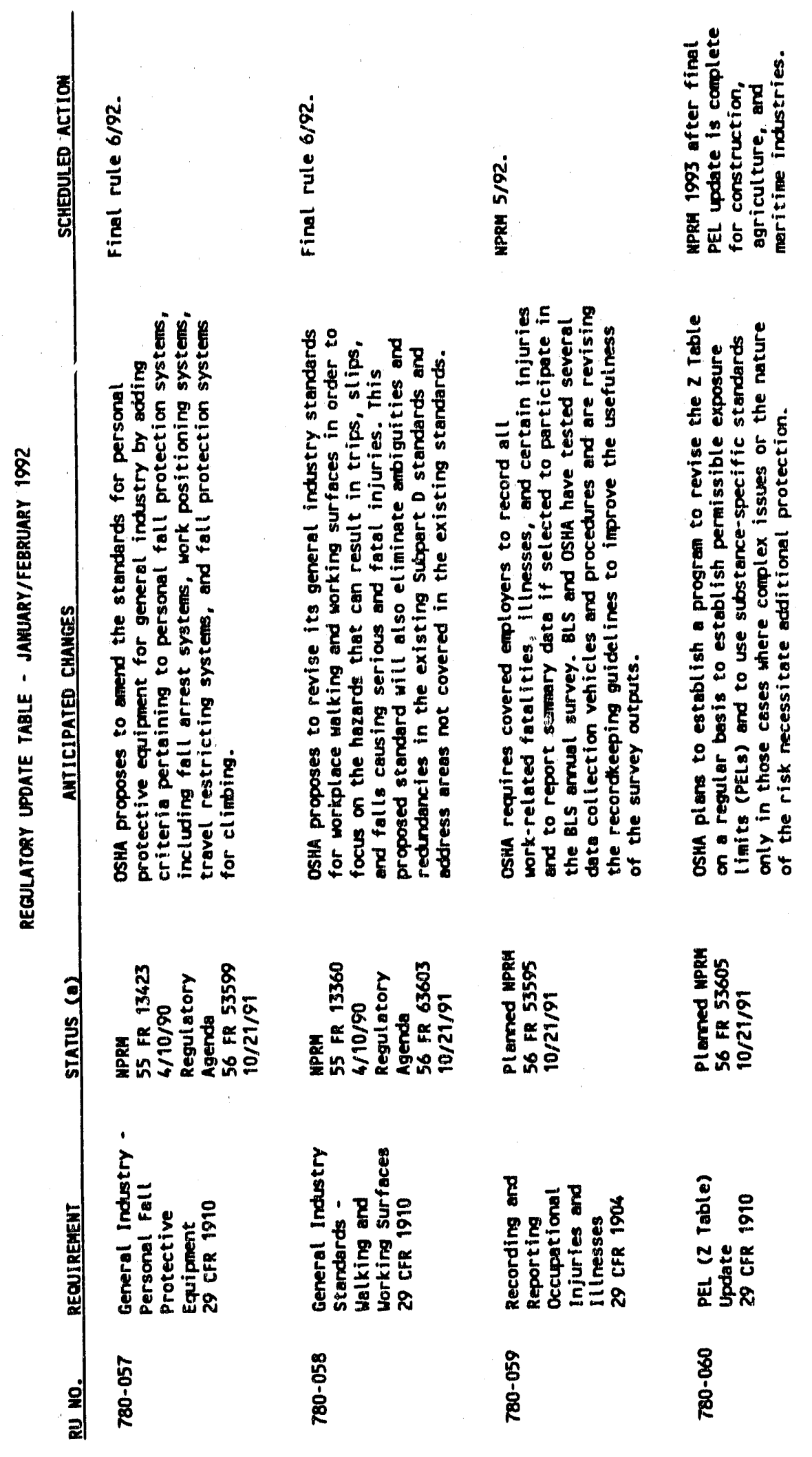




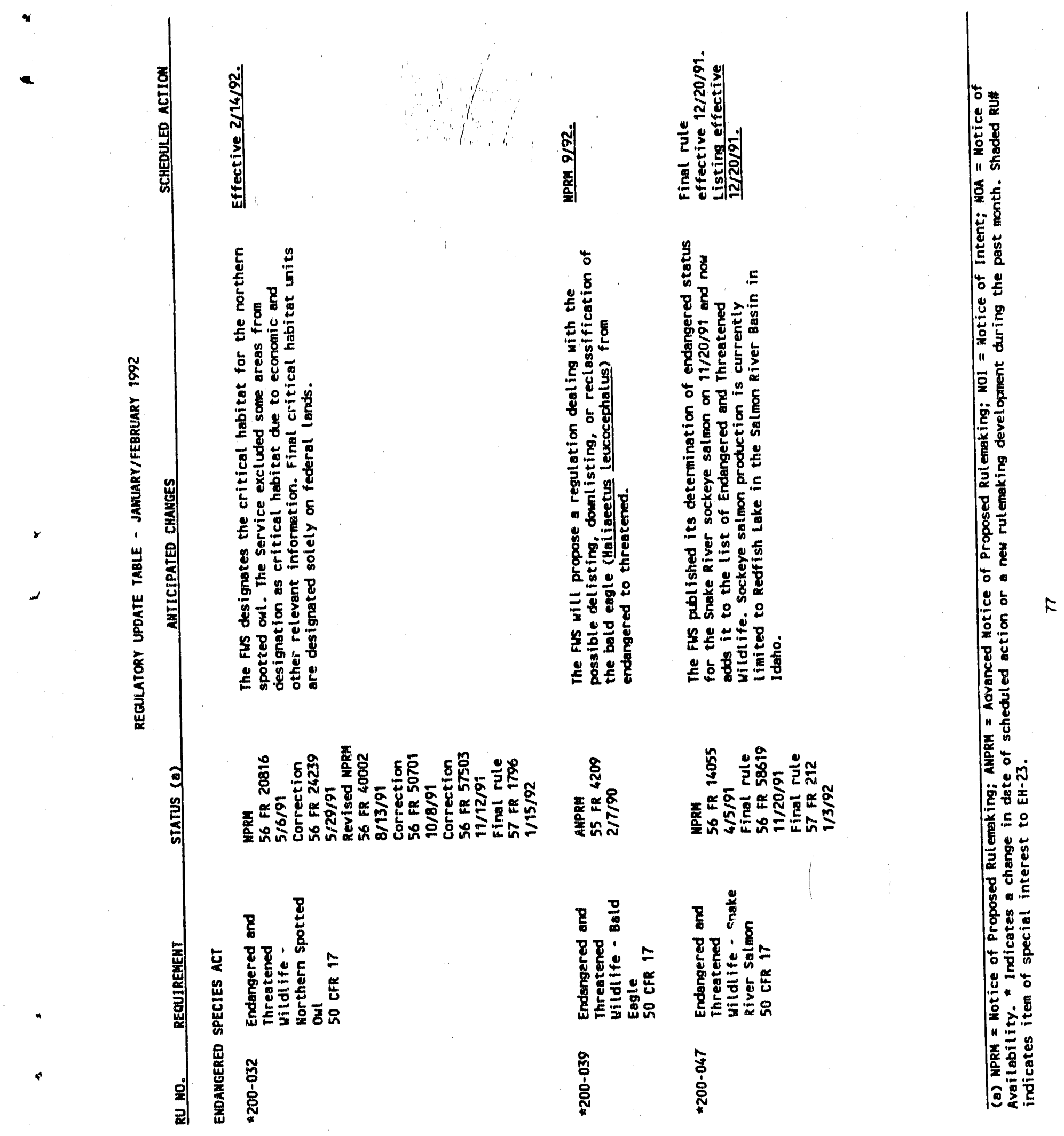




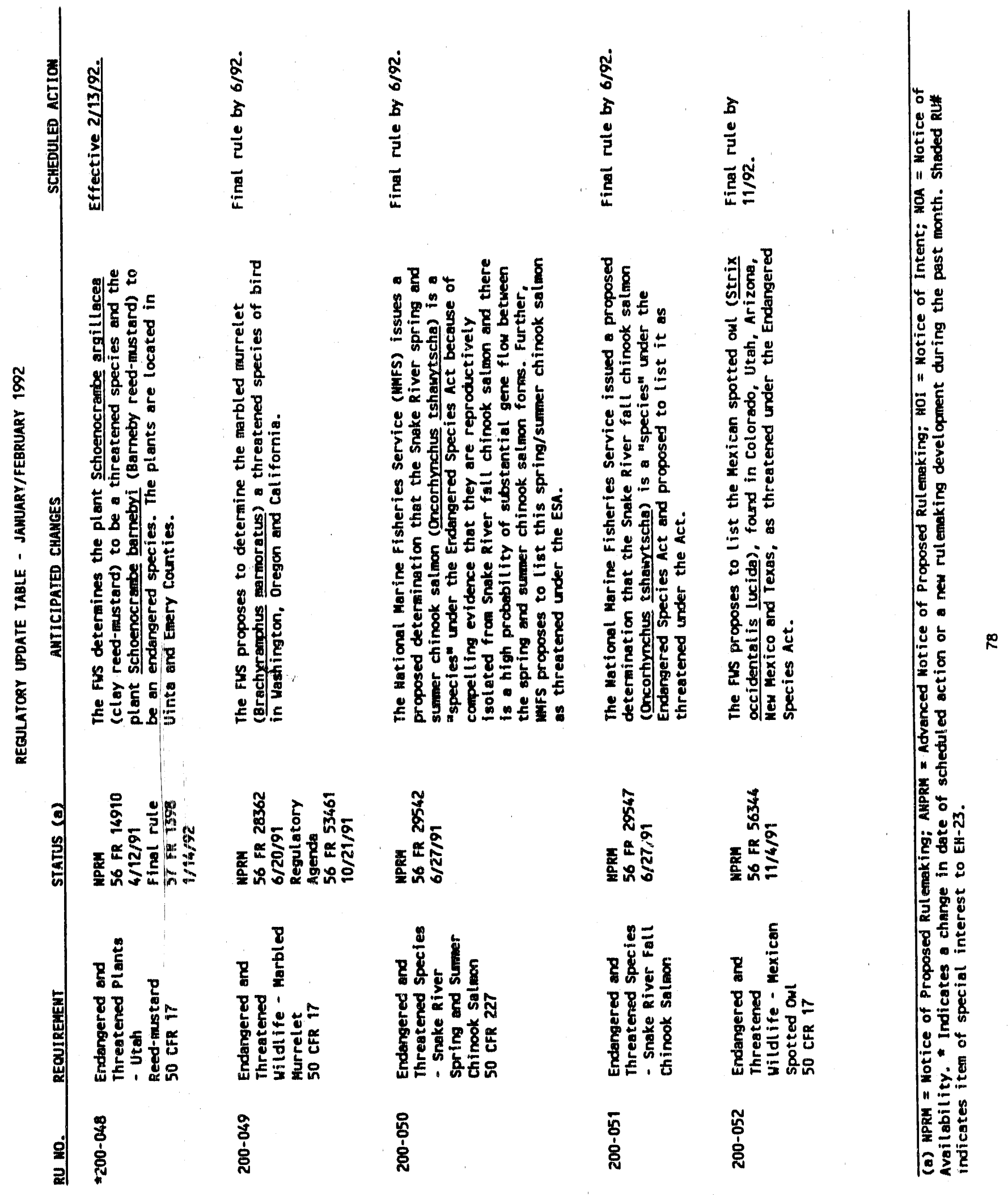




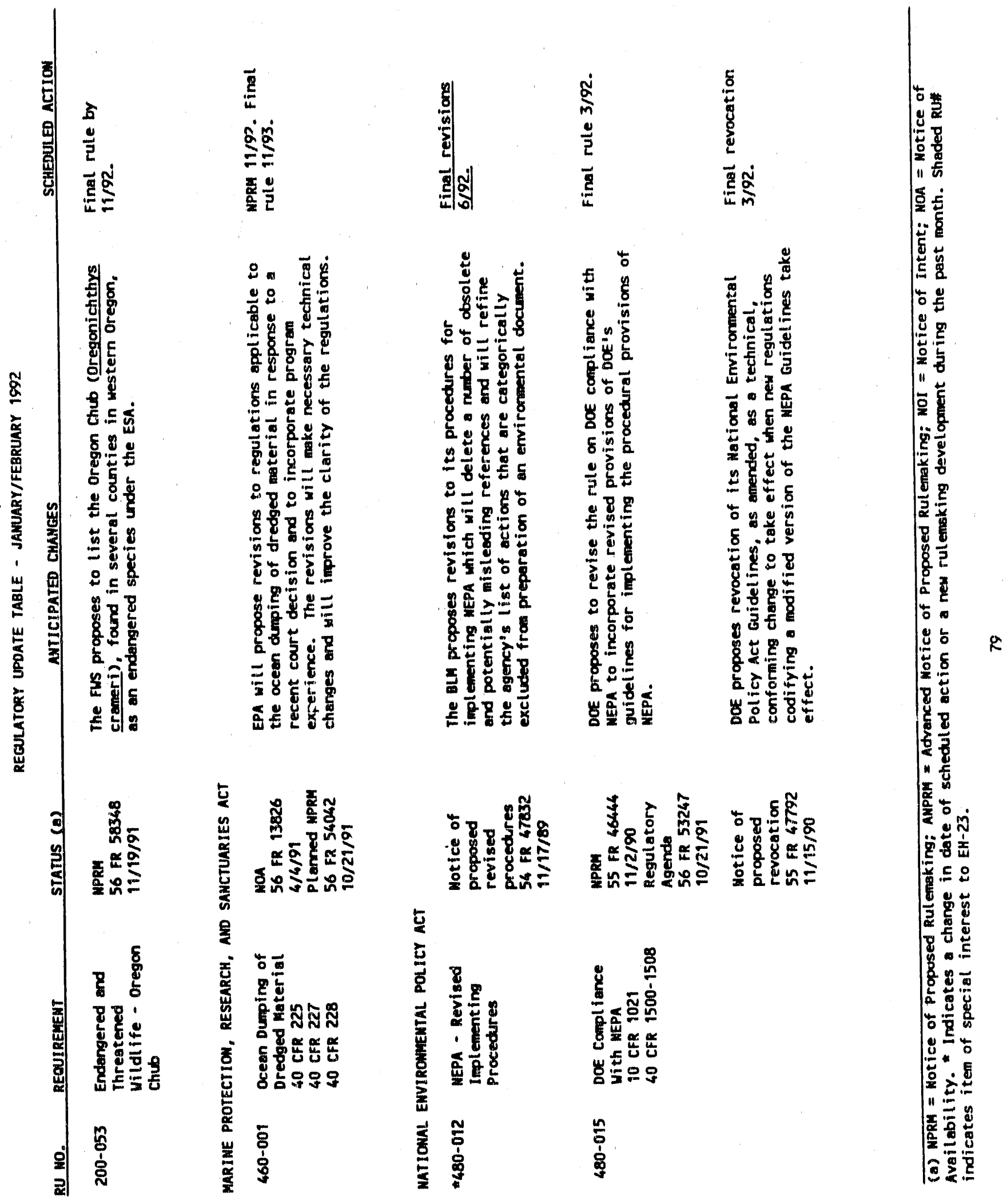




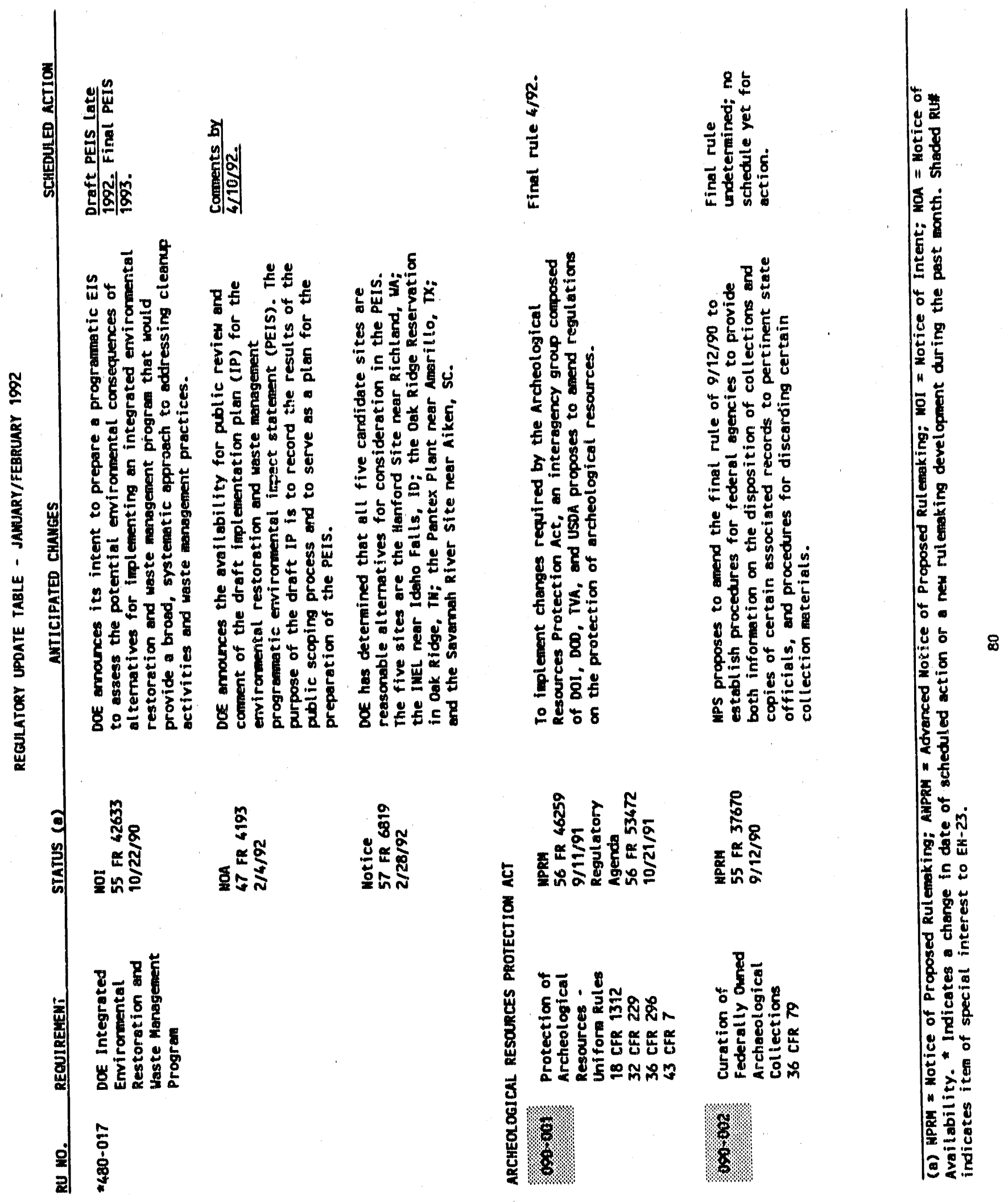




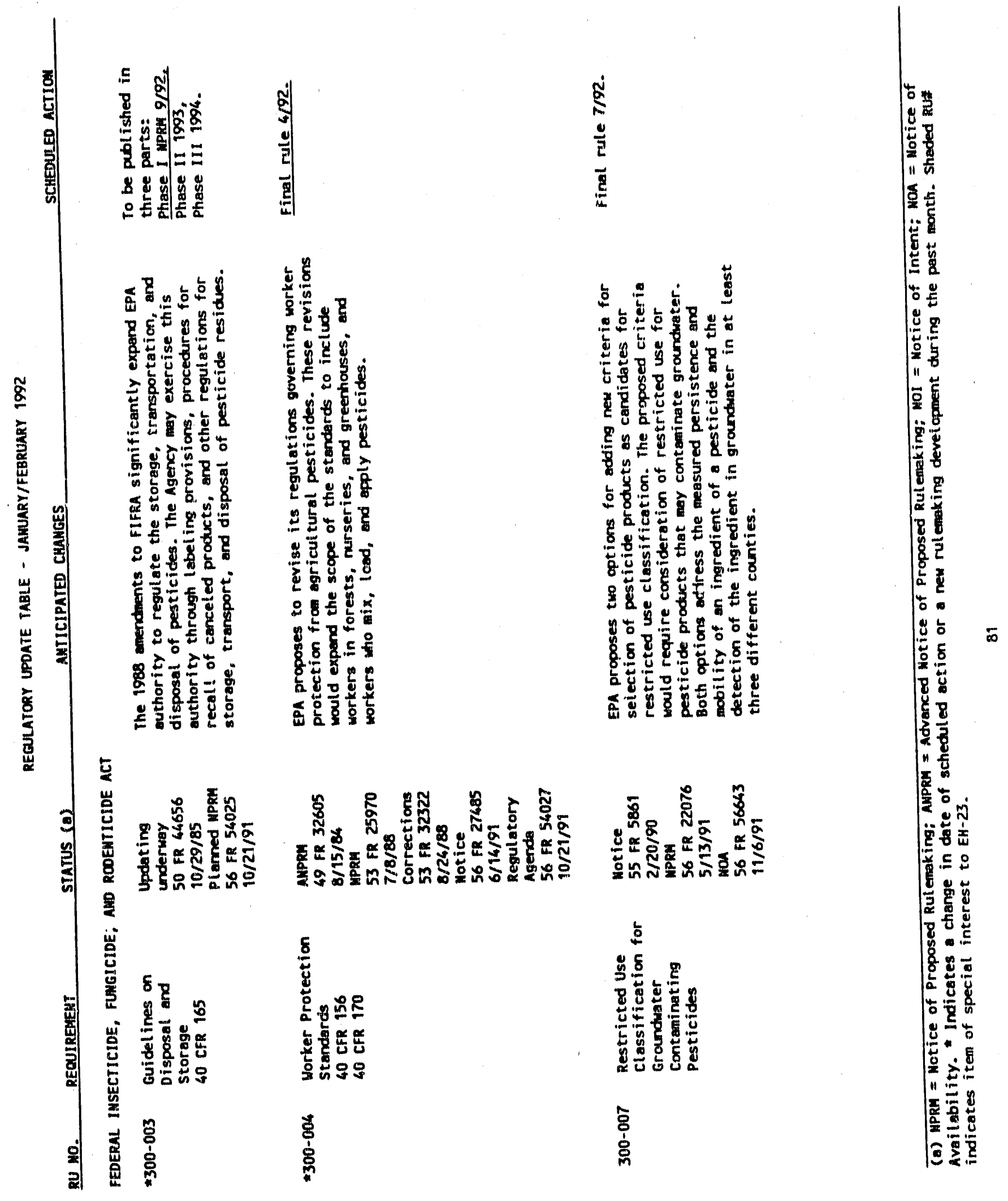




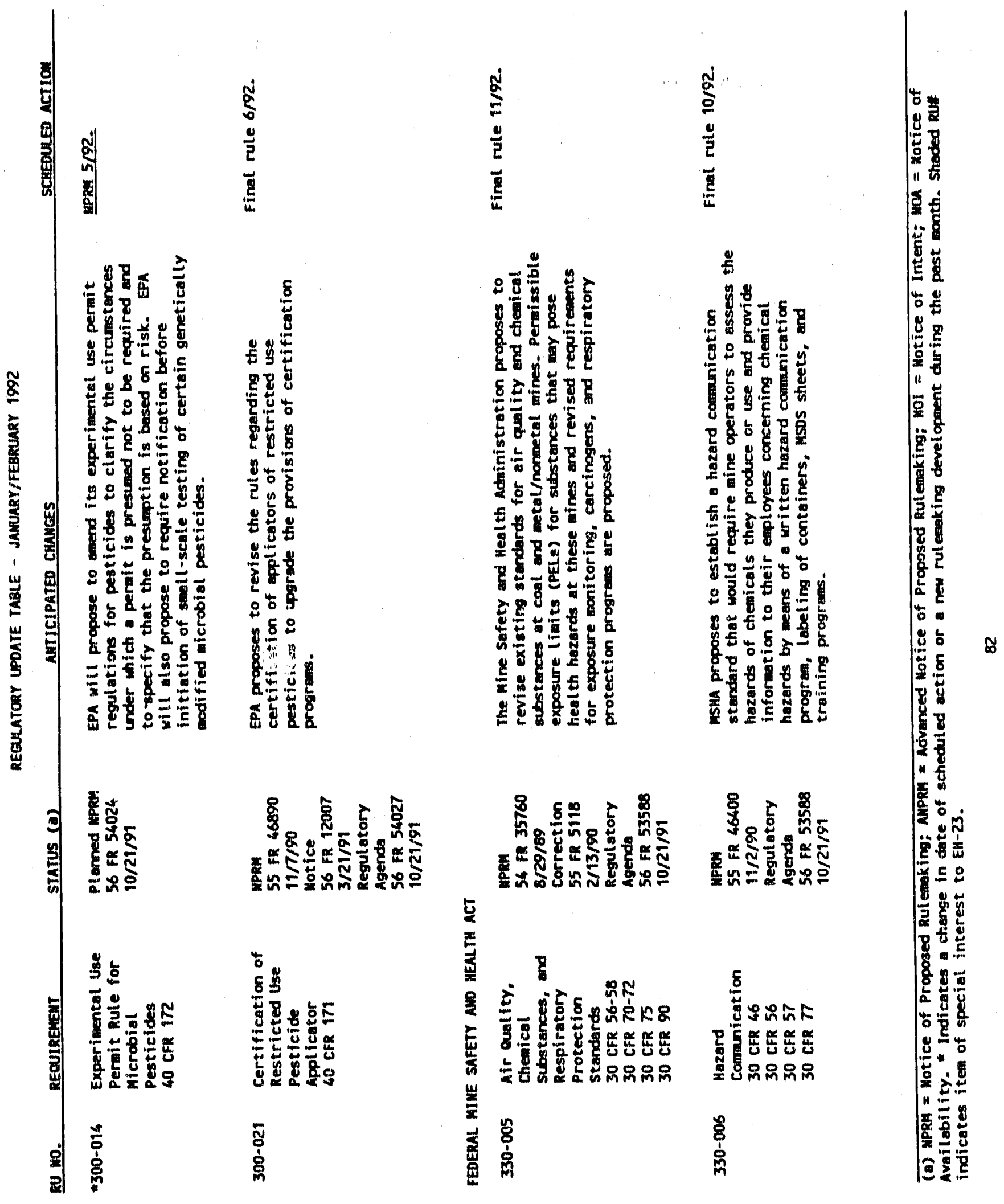




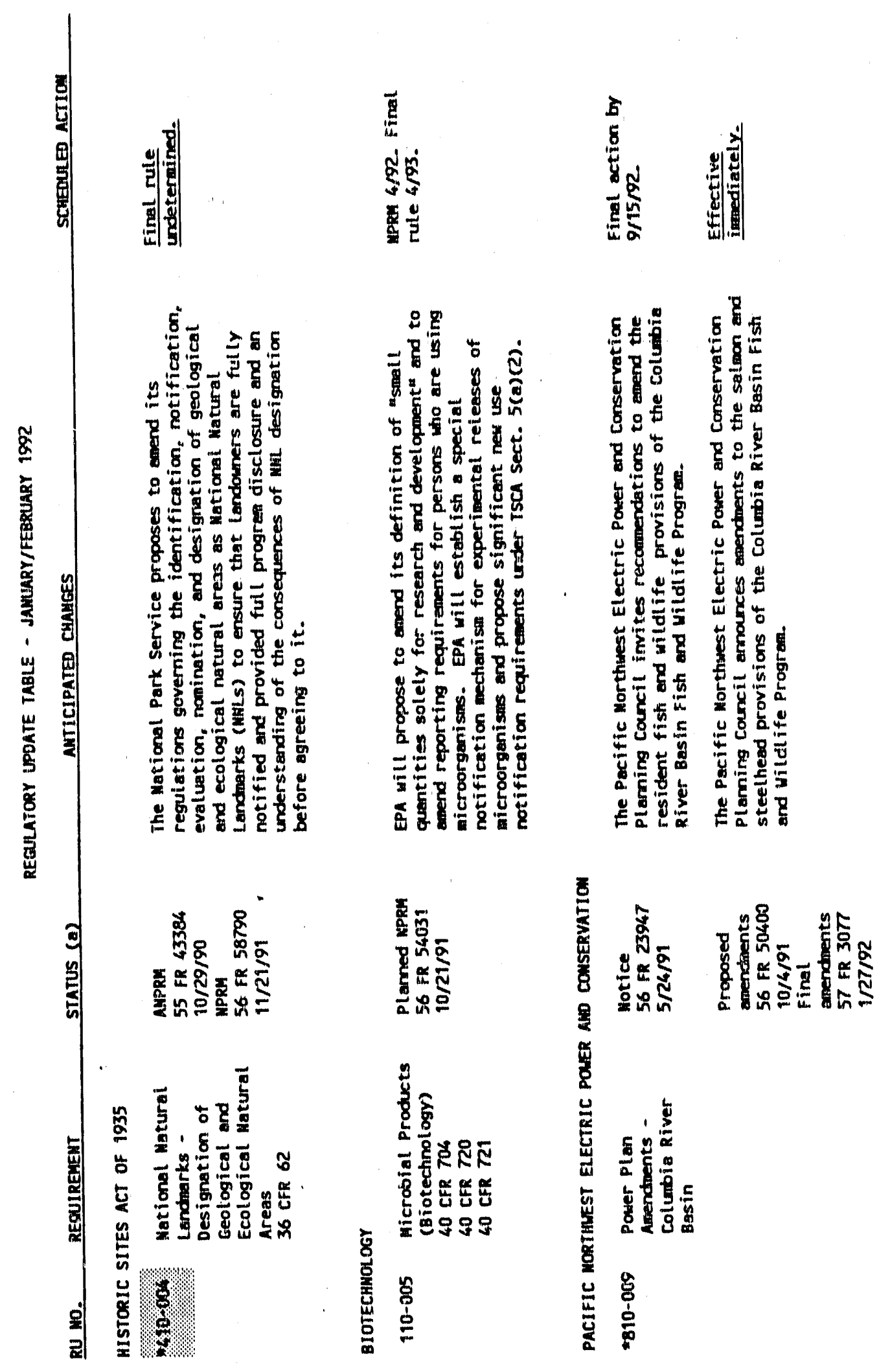



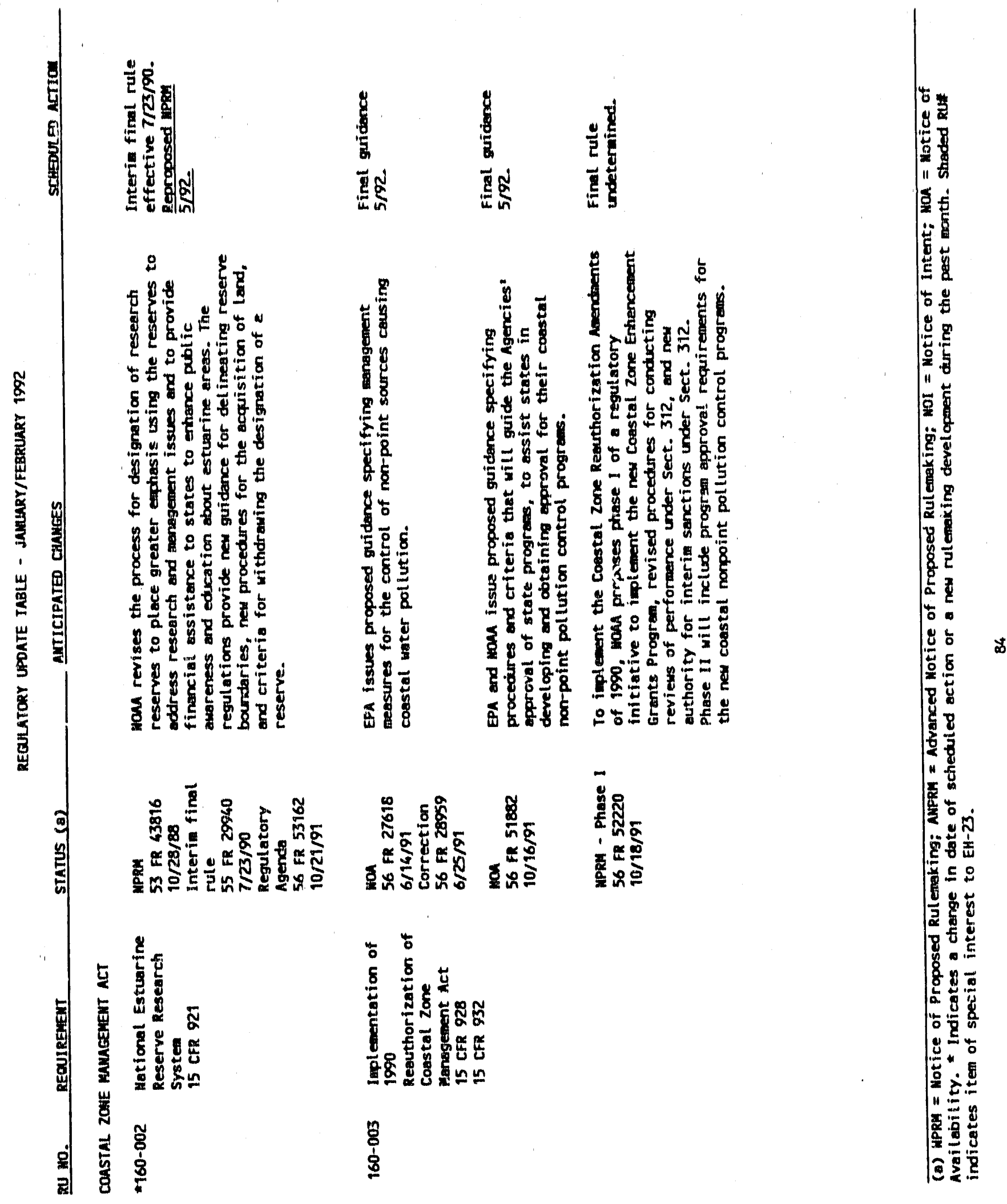


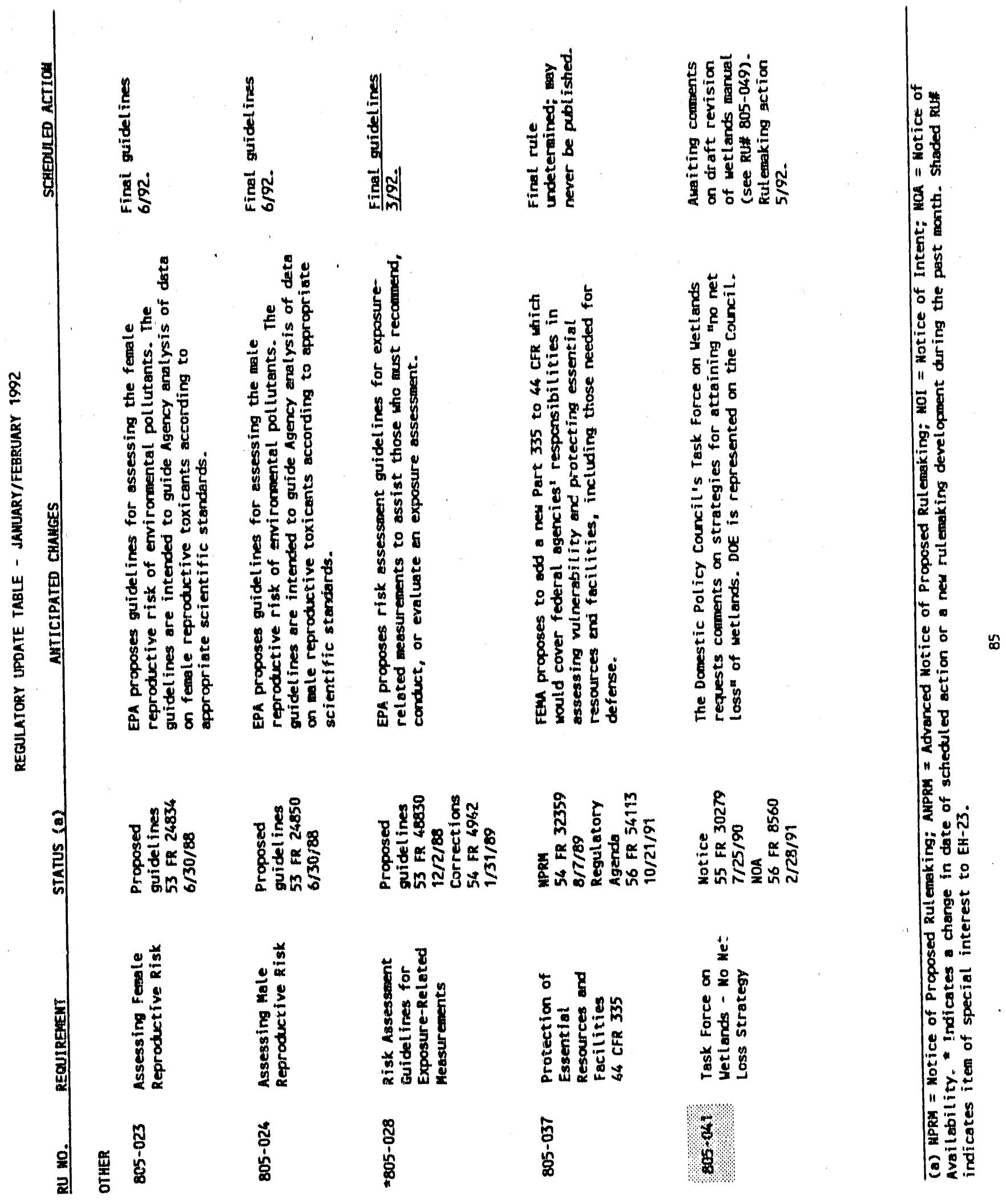




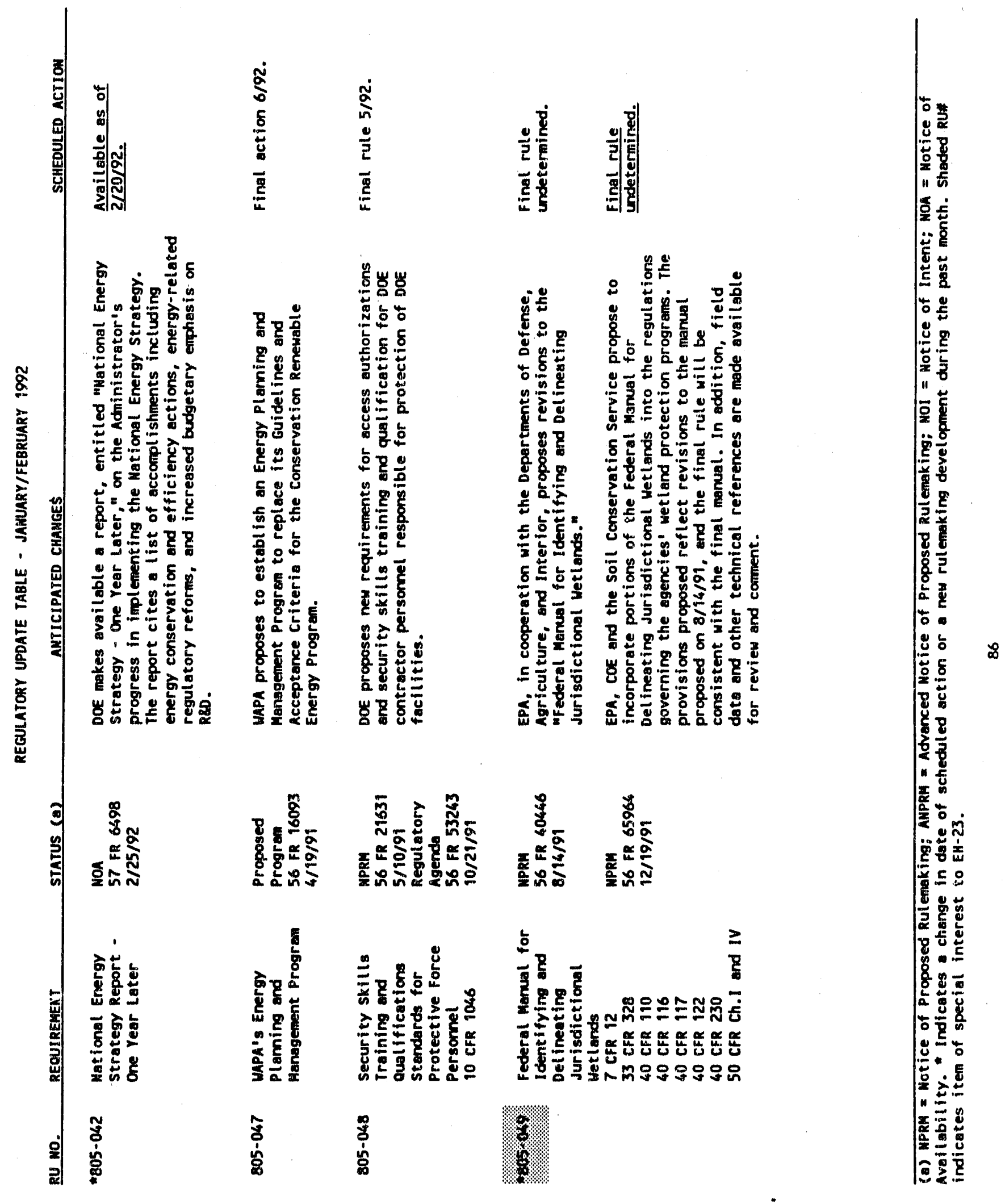




\section{IRIS DATABASE UPDATE}

A DOE EH-231 Memorandum entitled Integrated Risk Information System (IRIS) and Availability of Chemical Risk Assessment Guidance was distributed in June 1991 to DOE Program Offices and Field Organizations. Attachment 1 to the memorandum, an ORNL-ESD report discussing access to and utilization of the IRIS online database, included "Table 1.

IRIS Chemicals With Reference Doses and Carcinogen Assessments." The IRIS database has been updated extensively since that table was generated. The following table is the monthly updated version of Table 1 including all the chemicals listed in Table 1 along with additions/changes to the database as of February 28, 1992. Additions/changes are shaded for ease of identification. Future issues of the Environmental Regulatory Update Table will include updates to the IRIS database during the previous month. Use only the mast current version of the table. 


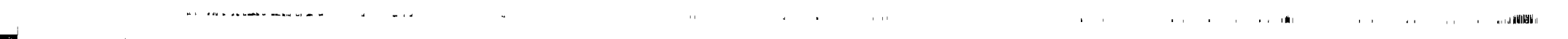




\section{IRIS CHEMICALS WITH REFERENCE DOSES AND/OR CARCINOGEN ASSESSMENTS}

(Values current through February 28, 1992. There have been no changes to the IRIS values since the December issue of the Update Table. Use only the most current version of this table.)

\begin{tabular}{|c|c|c|c|c|c|c|}
\hline NAME & CASRN & $\begin{array}{c}\text { ORAL } \\
\text { RD } \\
(m g / k / d y y)\end{array}$ & $\begin{array}{c}\text { INHAL } \\
\text { RrC } \\
(\mathrm{mg} / \mathrm{cu} \mathrm{m})\end{array}$ & $\begin{array}{l}\text { EPA } \\
\text { GROUP }\end{array}$ & $\begin{array}{c}\text { ORAL } \\
\text { SLOPE } \\
\text { PACTOR } \\
\text { (mg/ky/day) }\end{array}$ & $\begin{array}{c}\text { INHAL } \\
\text { SLOPE } \\
\text { PACTOR } \\
(\text { (mg/\&g/day) } \\
\end{array}$ \\
\hline ACENAPHTHENE & $000083-32-9$ & $6 \mathrm{E}-2$ & .. & - & - & .. \\
\hline ACENAPHTHYLENE & $000208-96-8$ &.- & -. & $\mathrm{D}$ & - &. \\
\hline ACEPHATE & $030560-19-1$ & $4 \mathrm{E}-3$ & .. & $\mathrm{C}$ & $8.7 \mathrm{E}-3$ &.$\cdot$ \\
\hline ACETALDEHYDE & $000075 \div 07-0$ & - & $9 \mathrm{E}-3$ & $\mathrm{~B} 2$ & -- & -. \\
\hline ACETONE & $000067-64-1$ & $1 E-1$ & -. & $\mathrm{D}$ & $\cdots$ & -. \\
\hline ACETONITRILE & $000075-05-8$ & $6 \mathrm{E}-3$ & -. & $\cdots$ & $\cdots$ & .. \\
\hline ACETOPHENONE & $000098-86 \cdot 2$ & $1 \mathrm{E}-1$ & .- & D & - & - \\
\hline ACETYL CHLORIDE & $000075-36-5$ & - & .. & $D$ & - & - \\
\hline ACROLEIN & $000107-02-8$ & - & $2 \mathrm{E}-5$ & $\mathrm{C}$ & - & 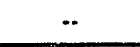 \\
\hline ACRYLAMIDE & $000079-06-1$ & $2 E-4$ & - & B2 & 4.5 & .. \\
\hline ACRYLIC ACID & $000079-10-7$ & 0.08 & $3 \mathrm{E}-4$ & - & -. & $\ldots$ \\
\hline ACRYLONITRILE & $000107-13-1$ & - & $2 \mathrm{E}-3$ & B1 & $5.4 \mathrm{E}-1$ & $-\cdot$ \\
\hline ADIPONITRILE & $000111-69-3$ & - & -- & $\mathrm{D}$ & -- & -- \\
\hline ALACHLOR & $015972-60-8$ & $1 \mathrm{E} \cdot 2$ & $\ldots$ & -. & - & -. \\
\hline ALAR & $001596-84-5$ & $1.5 \mathrm{E}-1$ & - & - & -- & - \\
\hline ALDICARB & $000116-06-3$ & $2 E-4$ & -. & $\mathrm{D}$ & -- & - \\
\hline ALDRIN & $000309-00-2$ & $3 \mathrm{E}_{-5}$ & - & B2 & $1.7 \mathrm{E}+1$ & - \\
\hline ALLY & $074223-64-6$ & 2.5E-1 & - & - & - & - \\
\hline ALLYL ALCOHOL & $000107-18-6$ & $5 \mathrm{E}-3$ & - & - & 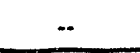 & -. \\
\hline ALLYL CHLORIDE & $000107-05-1$ & - & $1 \mathrm{E}-3$ & C & -. & .. \\
\hline ALUMINUM PHOSPHIDE & $020859-73-8$ & $4 \mathrm{E}-4$ & - & - & -. & $-\cdot$ \\
\hline AMDRO & $067485-29.4$ & $3 E-4$ & -. & - & - & -. \\
\hline AMETRYN & $000834-12-8$ & 9E-3 & -- & - & - & - \\
\hline AMINOFYRIDINE, 4- & $000504-24-5$ & - & -. & $\mathrm{D}$ & - & $\cdots$ \\
\hline AMITRAZ & $033089-61-1$ & $2.5 \mathrm{E}-3$ & - & - & - & -- \\
\hline AMMONLA & $007664-41-7$ & - & $1 \mathrm{E}-1$ & - & - &.- \\
\hline AMMONIUM ACETATE & $000631-61-8$ & - & - & D & .. & .. \\
\hline AMMONIUM SULFAMATE & $007773-06-0$ & $2 \mathrm{~F} \cdot 1$ & $\cdots$ & - & - & $\cdots$ \\
\hline ANILINE & $000062-53-3$ & - & $1 \mathrm{E}-3$ & B2 & $5.7 \mathrm{E}-3$ &. \\
\hline ANTHRACENE & $000120-12-7$ & $3 \mathrm{E}-1$ &.- & - & -. & $\cdots$ \\
\hline
\end{tabular}




\begin{tabular}{|c|c|c|c|c|c|c|}
\hline NAME & CASRN & $\begin{array}{c}\text { ORAL } \\
\text { RRD } \\
\text { (mg/ky diyy) }\end{array}$ & $\begin{array}{c}\text { INHAL } \\
\text { RrC } \\
\text { (mos } m \text { ) }\end{array}$ & $\begin{array}{c}\text { EPA } \\
\text { GROUP }\end{array}$ & $\begin{array}{c}\text { ORAL } \\
\text { SLOPE } \\
\text { PACTOR } \\
\text { (mgkg/day) } \\
\end{array}$ & $\begin{array}{c}\text { INHAL } \\
\text { SLOPE } \\
\text { FACTOR } \\
\text { (m/k/k/day) }\end{array}$ \\
\hline ANTIMONY (ME'TALLIC) & $007440-36-0$ & $4 E-4$ & -. & $\cdots$ & - & -. \\
\hline APOLLO & $074115-24-5$ & $1.3 \mathrm{E}-2$ & -. & $\mathrm{C}$ & - & .. \\
\hline ARAMITE & $000140-57-8$ & - & $-\infty$ & $\mathrm{B} 2$ & $2.5 \mathrm{E}-2$ & -. \\
\hline ARSIENIC, INORGANIC & $007440-38.2$ & $3 E-4$ & .. & A & -. & 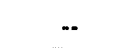 \\
\hline ASEBESTOS & $001332-21-4$ & - & - & A &.- & .. \\
\hline ASSURE & $076578-14-8$ & $9 \mathrm{E}-3$ & -- & $\mathrm{D}$ & -. & $\ldots$ \\
\hline ASULAM & $003337-71-1$ & $5 \mathrm{E}-2$ & - & $\cdots$ & -. & -- \\
\hline ATRAZINE & $001912-24-9$ & SE-3 & -- & - & - & - \\
\hline AVERMECTIN B1 & $065195.55-3$ & $4 E-A$ & -. & -. &.- & $\ldots$ \\
\hline AZOBENZENE & $000103-33-3$ & - & .. & B2 & $1.1 \mathrm{E}-1$ & -. \\
\hline BARIUM & $007440-39-3$ & $7 \mathrm{E}-2$ & - & - & .. & -- \\
\hline BARIUM CYANIDE & $000542-62-1$ & - & -- & - & - & $\cdot-$ \\
\hline BAYGON & $000114-26-1$ & $4 \mathrm{E}-3$ & -- & - & - & -- \\
\hline BAYLETON & $043121-43-3$ & $3 \mathrm{E}-2$ & - & - & -. & -- \\
\hline BAYTHROID & $068359-37.5$ & 2.5E-2 & - & -- & -. &.- \\
\hline BENEFIN & $001861-40-1$ & $3 E-1$ & -. & .. & -. & -- \\
\hline BENOMYL & $017804-35-2$ & $5 E \cdot 2$ & -. & - & -. & -. \\
\hline BENTAZON & $025057-89-0$ & $2.5 E-3$ & -- & -. & - & -- \\
\hline BENZ(A)ANTHRACENE & $000056-55-3$ & - & - & B2 & -- & -. \\
\hline BENZALDEHYDE & $000100-52-7$ & 1E-1 & .. & .. & -. & .- \\
\hline BENZENE: & $000071-43-2$ & - & -- & $\mathbf{A}$ & $2.9 \mathrm{E}-2$ & .. \\
\hline BENZIDINE & $000092-87-5$ & $3 E-3$ & - & $\mathbf{A}$ & $2.3 E+2$ & $2.3 E+2$ \\
\hline BENZO(A)PYRENE & $0.00050-32-8$ & - & -- & $\mathbf{B 2}$ & $\cdots$ & -. \\
\hline BENZO(B)FLUORANTHENE & $000205-99-2$ & - & - & B2 & -- & -. \\
\hline BENZO(G,H,I)PERYLENE & $000191-24-2$ & - & $\ldots$ & D & - & $-\cdot$ \\
\hline BENZOIC ACID & $000065-85-0$ & 4 & -. & $\mathrm{D}$ & - & .. \\
\hline BENZOTRICHLORIDE & $000098-07-7$ & - & - & B2 & $1.3 E+1$ & -- \\
\hline BENZOIKIJFLUORANTHENE & $000207-08-9$ & - & -. & B2 & - & - \\
\hline BENZYL CHLORIDE & $000100-\$ 4-7$ & - & - & B2 & $1.7 \mathrm{E}-1$ & -. \\
\hline BERYLLIUM & $007440-41-7$ & SE-3 & - & $\mathbf{B 2}$ & 4.3E-0 & .. \\
\hline BIDRIN & $000141-66-2$ & $1 E-4$ & - & -. & -- & -. \\
\hline BIPHENTHRIN & $082657-04-3$ & $1.5 \mathrm{E}-2$ & -- & - & $-\cdot$ & $\therefore$ \\
\hline BIPHENYL, 1,1'- & $000092-52-4$ & SE-2 & -- & $\mathrm{D}$ & $\ldots$ & $-\cdot$ \\
\hline BIS(2-CHLOROISOPROPYL) ETHER & $039638-32-9$ & 4E-2 & - & - & $\cdots$ & - \\
\hline
\end{tabular}




\begin{tabular}{|c|c|c|c|c|c|c|}
\hline NAME & CASRN & $\begin{array}{c}\text { ORAL } \\
\text { RD } \\
(\mathbf{m} z / / g / d y y)\end{array}$ & $\begin{array}{c}\text { INHAL } \\
\text { RRC } \\
\text { (m\&/cu } m \text { ) }\end{array}$ & $\begin{array}{c}\text { EPA } \\
\text { GROUP }\end{array}$ & $\begin{array}{c}\text { ORAL } \\
\text { SLOPE } \\
\text { PACTOR } \\
(\text { (mg/kg/day })^{-1}\end{array}$ & $\begin{array}{c}\text { INHAL } \\
\text { SLOPE } \\
\text { PACTOR } \\
\text { (mg/rg/day)" }\end{array}$ \\
\hline BIS(2-ETHYLHEXYL)PHTHALATE & $000117-81.7$ & $2 \mathrm{E}-2$ & $\cdots$ & B2 & $1.4 \mathrm{E}-2$ &.. \\
\hline BIS(CHLOROETHYL) ETHER & $000111-44-4$ & - & - & $\mathrm{B} 2$ & $1.1 \mathrm{E}+0$ & -. \\
\hline BIS(CHLOROMETHYL) ETHER & $000542-88-1$ & - & - & A & $2.2 \mathrm{E}+2$ & $-\cdot$ \\
\hline BISPHENOL A & 000080.05 .7 & SE-2 & - & 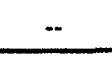 & - &.- \\
\hline BORON (BORON AND BORATES ONLY) & $007440-42-8$ & 9E-2 & $\ddot{-}$ & - & $\therefore$ & - \\
\hline BROMINATED DIBENZOFURANS & NO CASRN & - & -. & $\mathrm{D}$ & $-\cdot$ & 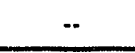 \\
\hline BROMOCHLOROMETHANE & $000074-97-5$ & - & - & $\mathrm{D}$ & $\ddot{-}$ & - \\
\hline BROMODICHLOROMETHANE ' & $-000075-27-4$ & $2 \mathrm{E}=2 \cdots$ & $\ddot{-}$ & B2 & $1.3 \mathrm{E}-1$ & 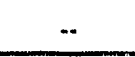 \\
\hline BROMODIPHENYL ETHER, P. & $000101-55-3$ & - & 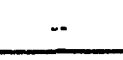 & $\mathrm{D}$ & 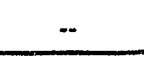 & - \\
\hline BROMOFORM & $000075-25 \cdot 2$ & $2 \mathrm{E}-2$ & - & $\mathrm{B} 2$ & $7.9 \mathrm{E}-3$ & - \\
\hline BROMOMETHANE & $000074.83-9$ & $1.4 \mathrm{E}-3$ &.- & $\mathrm{D}$ & 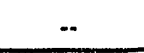 & $\ddot{-}$ \\
\hline BROMOTRICHLOROMETHANE & $000075-62-7$ & - & $\cdots$ & $\mathrm{D}$ & - & $\cdots$ \\
\hline BROMOXYNIL OCTANOATE & $001689-99-2$ & $2 \mathrm{E}-2$ & 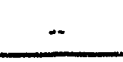 & $\ddot{-}$ & - & - \\
\hline BROMOXYNIL & $001689-84.5$ & $2 \mathrm{E}-2$ & - & -- & - & - \\
\hline BUTADIENE, 1,3- & $000106-99-0$ & - & $\ddot{-}$ & $\mathrm{B2}$ & - & - \\
\hline BUTANOL, 1 - & $000071 \cdot 36-3$ & $1 \mathrm{E}-1$ & - & D & - & - \\
\hline BUTYL BENZYL PHTHLATE, N- & $000085-68-7$ & $2 \mathrm{E}-1$ & $\cdots$ & $\mathrm{C}$ & $\cdots$ & $\because$ \\
\hline BUTYLATE & $002008-41-5$ & $5 \mathrm{E}-2$ & $\ldots$ & 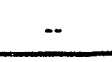 & - & - \\
\hline BUTYLPHTHALYL BUTYGLYCOLATE & $000085-70-1$ & $1 \mathrm{E}+0$ & - & $\ddot{-}$ & $\cdots$ & - \\
\hline CADMIUM & $007440-43-9$ & SE-4 & $\cdots$ & B1 & - & - \\
\hline CALCIUM CYANIDE & $000592-01-8$ & $4 \mathrm{E}-2$ & 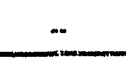 & - & - & - \\
\hline CAPROLACTAM & $000105-60-2$ & $5 E-1$ & - & - & - & $\cdots$ \\
\hline CAPTAFOL & $002425-06-1$ & $2 \mathrm{E} \cdot 3$ & 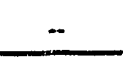 & $\ddot{-}$ & - & - \\
\hline CAPTAN & $000133-06-2$ & $1.3 \mathrm{E}-1$ & $\ddot{-}$ & $\cdots$ & $\ddot{-}$ & 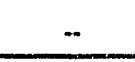 \\
\hline CARBARYL & $000063-25-2$ & $1 \mathrm{E}-1$ & - & - & - & $\therefore$ \\
\hline CARBOFURAN & $001563-66-2$ & 5E-3 & -. & $\cdots$ & -. & - \\
\hline CARBON DISULFIDE & $000075-15-0$ & $1 \mathrm{E}-1$ & 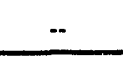 & $\because$ & - & - \\
\hline CARBON TETRACHLORIDE & $000056-23-5$ & $7 E-4$ & - & B2 & $1.3 \mathrm{E}-1$ & - \\
\hline CARBOSULFAN & $055285-14-8$ & $1 \mathrm{E}-2$ &.- & -- & 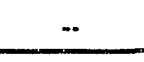 & - \\
\hline CARBOXIN & $005234-68-4$ & 1E-1 & - & - & $\cdots$ & - \\
\hline CHLORAL & 000075.87 .6 & $2 \mathrm{E}-3$ & $\ldots$ & -- &.- & - \\
\hline CHLORAMBEN & $000133.90-4$ & $1.5 \mathrm{E}-2$ & $\cdots$ & - & $\ldots$ & - \\
\hline CHLORANILINE, P. & $000106-47-8$ & $4 \mathrm{E},-3$ & $\ddot{-}$ & $\ddot{-}$ & - & - \\
\hline CHLORDANE & 000057.74 .9 & $6 \mathrm{E}-5$ & $\cdots$ & B2 & $1.3 E+0$ & $\cdots$ \\
\hline
\end{tabular}




\begin{tabular}{|c|c|c|c|c|c|c|}
\hline NAME & CASRN & $\begin{array}{c}\text { ORAL } \\
\text { RRD } \\
(m \& / k / d y y)\end{array}$ & $\begin{array}{c}\text { INHAL } \\
\text { RRC } \\
\text { (mg/w m) }\end{array}$ & $\begin{array}{l}\text { EPA } \\
\text { GROUP }\end{array}$ & 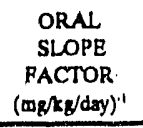 & $\begin{array}{c}\text { INHAL } \\
\text { SLOPE } \\
\text { PACTOR } \\
\text { (mg/kg/day) } \\
\end{array}$ \\
\hline CHLORIMURON-ETHYL & $090982-32-4$ & $2 \mathrm{E}-2$ & - & - & - & -. \\
\hline $\begin{array}{l}\text { CHLORINE CYANIDE } \\
\text { (CYANOGEN CHLORIDE) }\end{array}$ & $000506-77-4$ & $5 \mathrm{E}-2$ & - & $\cdots$ & $\cdots$ & $\cdots$ \\
\hline CHLORINE DIOXIDE & $010049-04-4$ & -- & $2 \mathrm{E}-4$ & - & -. & $\cdots$ \\
\hline $\begin{array}{l}\text { CHLORO-2-METHYL PROPANE, } 2 \text { - } \\
\text { (T-BUTYLCHLORIDE) }\end{array}$ & $000507-20-0$ & -- & - & $\mathrm{D}$ & -. & $-\cdot$ \\
\hline CHLOROACETOPHENONE, 2 - & $000532-27-4$ & - & $3 \mathrm{E}-5$ & - & $\therefore$ & .. \\
\hline CHLOROBENZENE & $000108-90-7$ & $2 E-2$ & - & D & $\cdots$ & 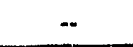 \\
\hline CHLOROBENZILATE & $000510-15-6$ & $2 \mathrm{E}-2$ & - & - &.- &.- \\
\hline CHLOROBUTANE, 1 - & 000109.69 .3 & - & $\ldots$ & $\mathrm{D}$ & - & -. \\
\hline CHLOROBUTANE, 2 - & $000078-86-4$ & - & - & $\mathrm{D}$ & 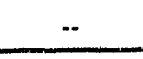 & $\ddot{-}$ \\
\hline CHLOROCYCLOPENTADIENE & $041851-50.7$ & - & - & $\mathrm{D}$ & $\because$ & $\cdots$ \\
\hline CHLOROETHOXY)METHANE, BIS(2- & $000111-91-1$ & - & 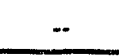 & D & - & .. \\
\hline CHLOROFORM & $000067-66 \cdot 3$ & $1 \mathrm{E}-2$ & -- & B2 & $6.1 \mathrm{E}-3$ & $\cdots$ \\
\hline CHLOROMETHTL METHYL ETHER & $000107-30-2$ & - & $\ldots$ & A & - & 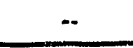 \\
\hline CHLORONAPHTHALENE, BETA- & $000091-58-7$ & $8 \mathrm{E}-2$ & - & - & - & $\therefore$ \\
\hline CHLOROPHENOL, 2 - & $000095-57-8$ & $5 \mathrm{E} \cdot 3$ & - & - & - & -. \\
\hline CHLORO'TOLUĖNE, 0- & $000095-49-8$ & $2 \mathrm{E}-2$ & - & - & - & -. \\
\hline CHLORPROPHAM & $000101-21.3$ & $2 \mathrm{E}-1$ & - & - & - & - \\
\hline CHLORPYRIFOS & a) $2921-88.2$ & $3 \mathrm{E}-3$ & .. & -. & -. & - \\
\hline CHLORSULFURON & $064902-72-3$ & $5 \mathrm{E}-2$ & - & - & $\cdots$ & - \\
\hline CHLOROTHALONIL & $001897-45-6$ & $1.5 E-2$ & - & - & - & - \\
\hline CHROMIUM(III) & $016065-83-1$ & $1 E+0$ & - & - & - & $\ddot{-}$ \\
\hline CHROMIUM(VI) & $018540-29.9$ & SE-3 & - & A & $\ldots$ & - \\
\hline CHRYSENE & $000218-01-9$ & - & - & $\mathrm{B} 2$ & $\cdots$ & - \\
\hline COAL TAR (COKE OVEN EMISSIONS) & $008007-45-2$ & - & - & A & -. & - \\
\hline COPPER & $007440-50-8$ & - & $\cdots$ & $\mathrm{D}$ & - & - \\
\hline COPPER CYANIDE & $000544-92-3$ & $5 E-3$ & - & .. & - & - \\
\hline CREOSOTE, COAL TAR & $008001-58-9$ & - & - & B1 & $\cdots$ & $\ddot{-}$ \\
\hline CROTONALDEHYDE & $000123-73-9$ & - & - & C & 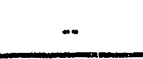 & - \\
\hline CYROMAZINE & 066215.27 .8 & $7.5 \mathrm{E}-3$ & - & -. & $\cdots$ & $\ddot{-}$ \\
\hline CUMENE & $000098-82-8$ & $4 \mathrm{E}-2$ & - & -. & - & 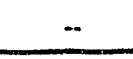 \\
\hline CYANAZINE & $0.21725-46-2$ & $2 \mathrm{E}-3$ & - & - & $\ddot{-}$ & - \\
\hline CYANIDE (CN*) & $000057-12-5$ & $2 \mathrm{E}-2$ & 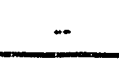 & $\mathrm{D}$ & $\cdots$ & - \\
\hline CUANOGEN & $000460-19.5$ & $4 \mathrm{E}-2$ & $\cdots$ & - & - & - \\
\hline
\end{tabular}




\begin{tabular}{|c|c|c|c|c|c|c|}
\hline NAME & CASRN & $\begin{array}{c}\text { ORAL } \\
\text { RID } \\
\text { (mg/xy/dxy) }\end{array}$ & $\begin{array}{c}\text { NHAL } \\
\text { RRC } \\
\text { (my/cu m) }\end{array}$ & $\begin{array}{l}\text { EPA } \\
\text { GROUP }\end{array}$ & $\begin{array}{c}\text { ORAL } \\
\text { SLOPE } \\
\text { PACTOR } \\
\text { (mg/xg/dsy)" }\end{array}$ & $\begin{array}{c}\text { INHAL } \\
\text { SLOPE } \\
\text { PACTOR } \\
\text { (mR/x/day)" }\end{array}$ \\
\hline CYANOGEN BROMIDE & $000506-68-3$ & $9 \mathrm{E} \cdot 2$ & - & $\cdots$ & $\cdots$ & $\cdots$ \\
\hline $\begin{array}{l}\text { CYANOGEN CHLORIDE } \\
\text { (CHLORINE CYANIDE) }\end{array}$ & $000506 \cdot 77-4$ & $5 \mathrm{E}-2$ & $\cdots$ & $\cdots$ & $-\cdot$ & $\cdots$ \\
\hline CYCLOHEXANONE & $000108-94-1$ & $5 E+0$ & $\cdots$ & $\cdots$ & $\cdots$ & $\cdots$ \\
\hline CYCLOHEXYLAMINE & $000108-91-8$ & $2 \mathrm{E}-1$ & $\cdots$ & $\cdots$ & $\cdots$ & $\ddot{-}$ \\
\hline CYHALOTHRIN/KARATE & $068085-85-8$ & $5 \mathrm{E}-3$ & $\cdots$ & - & $\cdots$ & $\cdots$ \\
\hline CYPERMETHRIN & $052315-07-8$ & $1 \mathrm{E}-2$ & $\therefore$ & $\cdots$ & 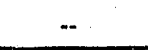 & $\ddot{-}$ \\
\hline DALAPON, SODIUM SALT & $000075-99-0$ & $3 \mathrm{E}-2$ & $\cdots$ & $\cdots$ & $\because$ & $\ddot{-}$ \\
\hline DDD & $000072-54-8$ & - & $\cdots$ & $\mathrm{B2}$ & $2.4 \mathrm{E}-1$ & $\cdots$ \\
\hline DDE & $000072-55.9$ & 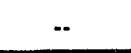 & $\therefore$ & B2 & $3.4 \mathrm{E}-1$ & $\cdots$ \\
\hline $\begin{array}{l}\text { DDT (DICHLORODIPHENYL- } \\
\text { TRICHLOROETHIANE, P,P'-) }\end{array}$ & $000050-29-3$ & $5 E-4$ & $\cdots$ & B2 & $3.4 \mathrm{E}-1$ & $\cdots$ \\
\hline DECABROMODIPHENYL ETHER & $0.01163-19-5$ & $1 \mathrm{E}-2$ & $\because$ & C & $\cdots$ & $\cdots$ \\
\hline DEMETON & $008065-48-3$ & $4 \mathrm{E}-5$ & $\cdots$ & 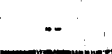 & - & $\because$ \\
\hline DI-(2-ETHYLHEXYL)ADIPATE & $000103-23-1$ & - & is & C & $1.2 \mathrm{E}-3$ & 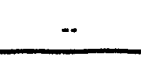 \\
\hline DIBENZ(A,H)ANTHRACENE & $000053-70-3$ & - & $\ldots$ & B2 & $\ddot{-}$ & 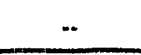 \\
\hline DIBENZOFURAN & $000132-64-9$ & - & $\cdots$ & D & 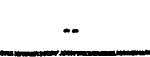 & 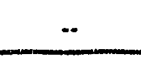 \\
\hline DIBROMOBENZENE, 1,4- & $000106-37-6$ & $1 \mathrm{E}-2$ & 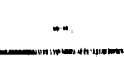 & $\because$ & $\cdots$ & -- \\
\hline DIBROMOCHLOROMETHANE & $000124-48-1$ & $2 \mathrm{E}-2$ & 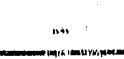 & 10 & B.4E:-2 & 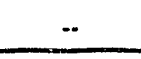 \\
\hline DIBROMODICHLOROMETHANE & $000594-18-3$ & - & $\cdots$ & m & $\ldots$ & $\cdots$ \\
\hline DIBROMO-3-CHLOROPROPANE, 1,2- & $000096-12-8$ & - & $2 E$ & -... & $\cdots$ & 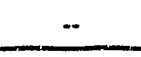 \\
\hline DIBROMODIPHENYL ETHER, P, P'- & $002050-47-7$ & - & $\ldots$ & $D$ & $\rightarrow$ & $\cdots$ \\
\hline DIBROMOETHANE, 1,2 & $000106-93-4$ & - & - & $\operatorname{lil}_{2} 2$ & Q.59.1 & 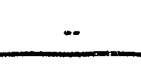 \\
\hline DIBUTYL PHTHALATE & $000084-74-2$ & $1 \mathrm{E}-1$ & - & A. & $\therefore$ & $\cdots$ \\
\hline DICAMBA & $001918-00.9$ & $3 E-2$ & $\cdots$ & $\cdots$ & $\cdots$ & - \\
\hline DICHLOROBENZENE, 1,2- & $000095-50-1$ & SE-2 & - & 9 & $+\cdots$ & - \\
\hline DICHLOROBENZENE, 1,3- & $000541-73.1$ & $\cdots$ & - & 2 & $\therefore$ & $\ddot{-}$ \\
\hline DICHLOROBENZIDINE, 3,3'- & $000091-94-1$ & - & $\cdots$ & 92 & $4.5 \mathrm{E} \cdots 1$ & $\cdots$ \\
\hline DICHLORODIFLUOROMETHANE & $000075-71-8$ & $2 \mathrm{E}-1$ & $\cdots$ & $\cdots$ & $m$ & 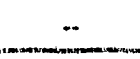 \\
\hline DICHLOROETHANE, 1,1- & $000075 \cdot 34-3$ & - & $\ldots$ & C & $\cdots$ & $\because$ \\
\hline DICHLOROETHANE, 1,2- & $000107-06-2$ & $\because$ & $\ldots$ & $B 2$ & 5i, inting & $\ldots$ \\
\hline DICHLOROETHYLENE, CIS-1,2,- & 000156.59 .2 & - & $\ldots$ & D & $\cdots$ & $\cdots$ \\
\hline DICHLOROETHYLENE, 1,2-T- & $000156-60-5$ & $2 \mathrm{E}-2$ & $\therefore$ & $\cdots$ & 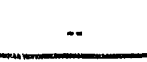 & $\cdots$ \\
\hline DICHLOROETHLYENE, 1,1- & $000075-35-4$ & DE.3 & $\cdots$ & $\mathbf{C}$ & $6 \mathrm{E}-1$ & .. \\
\hline
\end{tabular}




\begin{tabular}{|c|c|c|c|c|c|c|}
\hline NAME & CASRN & $\begin{array}{c}\text { ORAL } \\
\text { RID } \\
(m e / k z / d-y)\end{array}$ & $\begin{array}{c}\text { INHALL } \\
\text { R/C } \\
(m g / \omega) ~ m) ~\end{array}$ & $\begin{array}{l}\text { EPA } \\
\text { GROUP }\end{array}$ & $\begin{array}{c}\text { ORAL } \\
\text { SLOPE } \\
\text { PACTOR } \\
\text { (mg/kg/day) } \\
\end{array}$ & 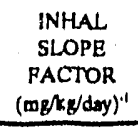 \\
\hline $\begin{array}{l}\text { DICHLOROMETHLANE } \\
\text { (METHYLENE CHLORIDE) }\end{array}$ & $000075-09-2$ & $6 \mathrm{E}-2$ & -. & B2 & $7.5 \mathrm{E}-3$ & $\therefore$ \\
\hline DICHLOROPHENOL, 2,4- & $000120-83-2$ & $3 E-3$ & - & $\ldots$ & - & - \\
\hline DICHLOROPHENOXYACETIC ACID, 2,4- & $000094-75-7$ & $1 \mathrm{E}-2$ & - & - & - & 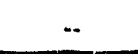 \\
\hline $\begin{array}{l}\text { DICHLOROPHENOXY)BUTYRIC ACID, } \\
\text { 4- }(2,4-\end{array}$ & $000094-82-6$ & $8 \mathrm{E}-3$ & -. & -- & -- & $\cdots$ \\
\hline DICHLOROPROPANE, 1,2- & $000078-87-5$ & - & 4E-3 & - & $\ldots$ & - \\
\hline DICHLOROPROPANOL, 2,3- & $000616-23-9$ & $3 \mathrm{E}-3$ & $\cdots$ & - & .. & $\cdots$ \\
\hline DICHLOROPROPENE, 1,3. & $000542-75-6$ & $3 E-4$ & $2 \mathrm{E}-2$ & B2 & - & $\ddot{-}$ \\
\hline DICHLORVOS & $000062.73-7$ & $8 \mathrm{E}-4$ & - & $\mathrm{B2}$ & $2.9 \mathrm{E}-1$ & 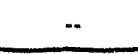 \\
\hline DICOFOL & $000115 \cdot 32-2$ & - & .. & $\mathrm{C}$ & $4.4 \mathrm{E}-1$ &.- \\
\hline DIELDRIN & $000060-57-1$ & $5 \mathrm{E} \cdot 5$ & -. & $\mathrm{B} 2$ & $1.6 \mathrm{E}+1$ & $\cdots$ \\
\hline DIETHYL PHTHALATE & $000084-66-2$ & $8 \mathrm{E}-1$ & .. & $\mathrm{D}$ & - & $-\cdot$ \\
\hline DIFENZOQUAT & $0432222-48-6$ & $8 \mathrm{E} \cdot 2$ &. & -. & - & $-\cdot$ \\
\hline DIFLUBENZURON & $035367-38.5$ & $2 \mathrm{E}-2$ & 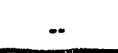 & - & - & $\cdots$ \\
\hline DIISOPROYL METHYLPHOSPHONATE & $001445-75-6$ & $8 \mathrm{E}-2$ & - & $\mathrm{D}$ & - & 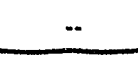 \\
\hline DIMETHIPIN & $055290.64-7$ & $2 \mathrm{E}-2$ & - & $\mathrm{C}$ & - & $\cdots$ \\
\hline DIMETHOATE & 000060.51 .5 & $2 \mathrm{E}-4$ & .. & - & - & $\cdots$ \\
\hline DIMETHYLANILINE, N,N. & $000121-69-7$ & $2 \mathrm{E}-3$ & .. & - & - & $\ddot{-}$ \\
\hline DIMETHYLFORMAMIDE & $000068-12-2$ & - & $3 \mathrm{E}-2$ & - & 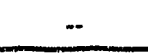 & $-\cdot$ \\
\hline DIMETHYLPHENOL, 2,6- & $000576-26-1$ & $6 \mathrm{E}-4$ & - & - & $\because$ & - \\
\hline DIMETHYLPHENOL, 2,4- & $000105-67-9$ & $2 \mathrm{E}-2$ & 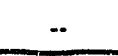 & - & - & - \\
\hline DIMETHYLPHENOL, 3,4- & $000095-65-8$ & $1 \mathrm{E}-3$ & - & - & $\ldots$ & $-\cdot$ \\
\hline DIMETHYLPHTHALATE & $000131-11-3$ & - & - & $\mathrm{D}$ & - & $\cdots$ \\
\hline DIMETHYL SULFATE & $000077-78-1$ & - & - & B2 & $\ddot{-}$ & - \\
\hline DIMETHYLTEREPHTHALATE & $000120-61-6$ & 1E-1 & - & - & - & $\cdots$ \\
\hline DINITRO-O-CYCLOHEXYL PHENOL, 4,6- & $000131-89.5$ & 2E-3 & - & $\ldots$ & -. &.. \\
\hline DINITROBENZENE, 1,3- & $000099-65-0$ & $1 \mathrm{E}-4$ & - & $\mathrm{D}$ & $\cdots$ & 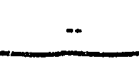 \\
\hline DINITROPHENOL, 2,4 . & $000051.28-5$ & 2E-3 & - & -. & - & $\ldots$ \\
\hline DINITROTOLUENE MLXTURE & NO CASRN & - & -- & B2 & $6.8 \mathrm{E}-1$ & -. \\
\hline DINOSEB & $000088-85-7$ & $1 \mathrm{E}-3$ & - & $\mathrm{D}$ & - & $\therefore$ \\
\hline DIOXANE, 1,4- & $000123-91-1$ & - & - & B2 & $1.1 \mathrm{E}-2$ & - \\
\hline DIPHENAMID & $0.00957 .51-7$ & $3 E-2$ & - & - & - & - \\
\hline DIPHENYLAMINE & $000122-39.4$ & $2.5 \mathrm{E}-2$ & - & - & - & - \\
\hline DIPHENYLHYDRAZINE, 1,2- & $000122-66-7$ & - & -. & $\mathrm{B} 2$ & $8.0 \mathrm{E}-1$ & $\cdots$ \\
\hline
\end{tabular}




\begin{tabular}{|c|c|c|c|c|c|c|}
\hline NAME & CASRN & $\begin{array}{c}\text { ORAL } \\
\text { RRD } \\
(\mathbf{m g / k / d} / \mathrm{my})\end{array}$ & $\begin{array}{c}\text { INH'AL } \\
\text { RRC } \\
(m / d w)\end{array}$ & $\begin{array}{c}\text { EPA } \\
\text { GROUP }\end{array}$ & $\begin{array}{c}\text { ORAL } \\
\text { SLOPE } \\
\text { PACTOR } \\
(\text { mg/kg/day) } \\
\end{array}$ & $\begin{array}{l}\text { INHAL } \\
\text { SLOPE } \\
\text { PACTOR } \\
(\text { (mg/tg/day) }\end{array}$ \\
\hline DIQUAT & $000085-00-7$ & $2.2 \mathrm{E}-3$ & - & 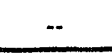 & 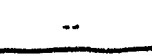 & $\cdots$ \\
\hline DISULFOTON & $000298-04-4$ & $4 \mathrm{E}-5$ & - & $\cdots$ & $\cdots$ & - \\
\hline DITHIANE, 1,4- & $000505-29 \cdot 3$ & - & - & $\mathrm{D}$ & 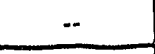 & $\cdots$ \\
\hline DIURON & $000330-54-1$ & $2 \mathrm{E}-3$ & $\because$ & - & $\because$ & 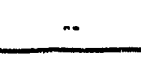 \\
\hline DODINE & $002439-10-3$ & 4E-3 & - & - & $\cdots$ & $\cdots$ \\
\hline ENDOSULFAN & $000115-29-7$ & $5 \mathrm{E}-5$ & - & - & 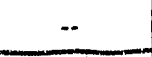 & $\ddot{-}$ \\
\hline ENDOTHALL & $000145-73-3$ & $2 \mathrm{E}-2$ & $\cdots$ & - & $=$ & $\therefore$ \\
\hline ENDRIN & $000072 \cdot 20-8$ & $3 E-4$ & - & $\mathrm{D}$ & $\therefore$ & - \\
\hline EPICHLOROHYDRIN & $000106-89-8$ & $2 \mathrm{E}-3$ & - & $\mathrm{B} 2$ & $9.9 \mathrm{E}-3$ & - \\
\hline EPTC & $000759-94-4$ & $2.5 \mathrm{E}-2$ & - & $\cdots$ & $\cdots$ & $\cdots$ \\
\hline ETHEPHON & $016672-87-0$ & SE-3 & - & -. & $\because$ & $\ddot{-}$ \\
\hline ETHION & $000563-12-2$ & $5 \mathrm{E}-4$ & - & $\ddot{-}$ & - & - \\
\hline ETHOXYETHANOL, 2 . & $000110-80-5$ & - & $2 \mathrm{E} \cdot 1$ & - & $\because$ & 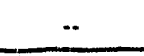 \\
\hline $\begin{array}{l}\text { ETHYL-P-NTTROPHENYL } \\
\text { PHOSPHONATE THIONOBENZENE } \\
\text { (EPN) }\end{array}$ & $002104-64-5$ & $1 \mathrm{E}-5$ & -. & -- & -- & -. \\
\hline ETHYL ETHER & $000060-29-7$ & $2 \mathrm{E}-1$ & - & - & - & - \\
\hline ETHYL CHLORIDE & $000075-00-3$ & - & $1 E+1$ & $\cdots$ & $\ddot{-}$ & - \\
\hline ETHYL ACETATE & $000141-78-6$ & 9E-1 & $\cdots$ & 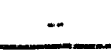 & $\ddot{-}$ & $\therefore$ \\
\hline ETHYLBENZENE & $000100-41-4$ & $1 \mathrm{E}-1$ & $1 E+0$ & D & $\because$ & $\because$ \\
\hline ETHYLENE THIOUREA & $000096-45-7$ & $8 \mathrm{E}-5$ & 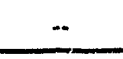 & 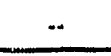 & - & - \\
\hline ETHYLENE GLYCOL & $000107-21-1$ & $2 \mathrm{E}+0$ & - & 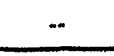 & $\because$ & $\cdots$ \\
\hline ETHYLPHTHALYL ETHYL GLYCOLATE & $000084-72-0$ & $3 E+0$ & - & .. & $\ddot{-}$ & $\because$ \\
\hline EXPRESS & $101200-48-0$ & $8 \mathrm{E}-3$ & - & - & $\cdots$ & $\cdots$ \\
\hline FENAMIPHOS & $022224.92-6$ & $2.5 \mathrm{E}-4$ & - & - & $\ddot{-}$ & $-\cdots$ \\
\hline $\begin{array}{l}\text { FENPROPATHRIN } \\
\text { (DANITOL) }\end{array}$ & $039515-41-8$ & $5 E-4$ & - & - & - & -. \\
\hline FLUOMETURON & $002164-17-2$ & $1.3 \mathrm{E}-2$ & - & - & - & $\cdots$ \\
\hline FLUORANTHENE & $000206-44-0$ & 4E-2 & $\cdots$ & D & $\cdots$ & - \\
\hline FLUORENE & $000086-73-7$ & $4 \mathrm{E}-2$ & 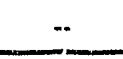 & $\mathrm{D}$ & $\ddot{-}$ & -. \\
\hline FLUORINE & $007782-41-4$ & $6 \mathrm{E}-2$ & $\cdots$ & $\therefore$ & -. & - \\
\hline FLURIDONE & $059756-60-4$ & $8 \mathrm{E}-2$ & - & $\cdots$ & - & 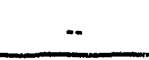 \\
\hline FLURPRIMIDOL & $056425-91.3$ & $2 \mathrm{E}-2$ & - & - & - & - \\
\hline FLUTOLANIL & $066332-96-5$ & $6 \mathrm{E}-2$ & $\cdots$ & 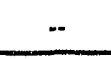 & $\cdots$ & - \\
\hline FLUVALINATE & $069409-94-5$ & $1 \mathrm{E}-2$ & - & 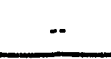 & $\ddot{-}$ & $\cdots$ \\
\hline
\end{tabular}




\begin{tabular}{|c|c|c|c|c|c|c|}
\hline NAME & CASRN & $\begin{array}{c}\text { ORAL. } \\
\text { RRD } \\
(m g / k g / \text { dey) }\end{array}$ & $\begin{array}{c}\text { INHAL } \\
\text { RSC } \\
\text { (mg/ed m) }\end{array}$ & $\begin{array}{l}\text { EPA } \\
\text { GROUP }\end{array}$ & $\begin{array}{c}\text { ORAL } \\
\text { SLOPE } \\
\text { FACTOR } \\
\text { (mg/kg/day)" }\end{array}$ & $\begin{array}{c}\text { INHLAL } \\
\text { SLOPE } \\
\text { PACTOR } \\
\text { (MR/Kg/doy)" }\end{array}$ \\
\hline FOLPET & $000133-07.3$ & $1 \mathrm{E}-1$ & $\cdots$ & B2 & $3.5 \mathrm{E} \cdot 3$ & $\cdots$ \\
\hline FOMESAFEN & $072128-02-0$ & -- & $\ldots$ & C & $1.9 \mathrm{E}-1$ & $\cdots$ \\
\hline FONOFOS & $000944-22-9$ & $2 \mathrm{E}-3$ & -- & -. & $\ldots$ & $\cdots$ \\
\hline FORMALDEHYDE & $000050-00-0$ & $2 \mathrm{E}-1$ & .. & B1 & $\cdots$ & $\cdots$ \\
\hline FORMIC ACID & $000064-18-6$ & - & -. & .- & .. &.- \\
\hline FOSETYLAL & $039148-24-8$ & $3 E+0$ & - & $\mathrm{C}$ & -- & -. \\
\hline FURAN & $000110-00.9$ & 1E-3 & - & - & - & - \\
\hline FURAZOLIDONE & $000067-45-8$ & - & $\cdots$ &.- & - & $-\cdot$ \\
\hline FURFURAL & $000098-01-1$ & $3 E-3$ & -- & .. & .. & -- \\
\hline FURMECYCLOX & $060568-05-0$ & -. & .. & $\mathrm{B} 2$ & $3.0 \mathrm{E}-2$ & - \\
\hline GLUFOSINATE-AMMONIUM & $077182-82-2$ & $4 \mathrm{E}-4$ & -- & - & -- & $\therefore$ \\
\hline GLYCIDALDEHYDE & 000765-34-4 & $4 \mathrm{E}-4$ & $\ldots$ & $\mathrm{B} 2$ & .. & -- \\
\hline GLYPHOSATE & $001071-83-6$ & $1 \mathrm{E}-1$ & -- & $\mathrm{D}$ & - & -- \\
\hline GOAL (OXYFLUORFEN) & $042874-03-3$ & 3E-3 & - & - & - & -- \\
\hline HALOXYFOP-METHYL & $069806-40.2$ & SE-5 & $\ldots$ & .. & -. & - \\
\hline HARMONY & $079277 \cdot 27-3$ & $1.3 \mathrm{E}-2$ & - & $-\cdot$ & -. & - \\
\hline HEPTACHLOR & $000076-44-8$ & $5 \mathrm{E}-4$ & -- & $\mathrm{B} 2$ & $4.5 \mathrm{E}+0$ & -- \\
\hline HEPTACHLOR EPOXIDE & 001024.57 .3 & $1.3 E \cdot 5$ & - & B2 & $9.1 \mathrm{E}+0$ & 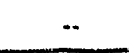 \\
\hline HEXABROMOBENZENE & $000087-82-1$ & $2 \mathrm{E}-3$ & - & -. &.- & $\cdots$ \\
\hline HEXABROMODIPHENYL ETHER & $036483-60-0$ & - & - & $\mathrm{D}$ & - & $\ddot{-}$ \\
\hline HEXACHLOROBENZENE & $000118-74-1$ & $8 \mathrm{E}-4$ & - & B2 & 1.6 & $\ddot{-}$ \\
\hline HEXACHLOROBUTADIENE & $000087-68-3$ & $2 \mathrm{E}-3$ & - & C & $7.8 \mathrm{E}-2$ & - \\
\hline HEACACHLOROCYCLOHEXANE, ALPHA- & $000319-84-6$ & - & - & B2 & $6.3 \mathrm{E}+0$ & - \\
\hline HEXACHLOROCYCLOHEXANE, BETA & $000319-85-7$ & - & $\ldots$ & C & $1.8 \mathrm{E}+0$ & - \\
\hline $\begin{array}{l}\text { HEXACHLOROCYCLOHEXANE, } \\
\text { GAMMA- }\end{array}$ & $000058-89-9$ & $3 \mathrm{E}-4$ & - & - & -- & $\cdots$ \\
\hline $\begin{array}{l}\text { HEXACHLOROCYCLOHEXANE, } \\
\text { EPSILON- }\end{array}$ & $006108-10-7$ & - &.- & D & - & $\cdots$ \\
\hline HEXACHLOROCYCLOHEXANE, DELTA- & $000319-86-8$ & - & - & D & $\cdots$ & $\because$ \\
\hline $\begin{array}{l}\text { HEXACHLOROCYCLOHEXANE, } \\
\text { TECHNICAL. }\end{array}$ & $000608-73-1$ & - & - & B2 & $1.8 \mathrm{E}+0$ & $-\cdot$ \\
\hline HEXACHLOROCYCLOPENTADIENE & $000077-47-4$ & 7E-3 & - & $\mathrm{D}$ & - & $\cdots$ \\
\hline $\begin{array}{l}\text { HEXACHLORODIBENZO-P-DIOXIN } \\
\text { MDXTURE }\end{array}$ & $019408-74-3$ & - & - & $\mathrm{B2}$ & $6.2 E+3$ & -- \\
\hline HEXACHLOROETHANE & $000067-72-1$ & $1 \mathrm{E}-3$ & - & $\mathrm{C}$ & $1,4 \mathrm{E}-2$ & - \\
\hline HEXACHLOROPHENE & $000070-30-4$ & $3 \mathrm{E}-4$ & - & - &.- & - \\
\hline
\end{tabular}




\begin{tabular}{|c|c|c|c|c|c|c|}
\hline NAME & CASRN & $\begin{array}{c}\text { ORAL } \\
\text { RRD } \\
(m g k g / d x y)\end{array}$ & $\begin{array}{l}\text { INHAL } \\
\text { R/C } \\
\text { (mg/cu m) }\end{array}$ & $\begin{array}{l}\text { EPA } \\
\text { GROUP }\end{array}$ & $\begin{array}{c}\text { ORAL } \\
\text { SLOPE } \\
\text { PACTOR } \\
\text { (mg/Kg/day)" } \\
\end{array}$ & $\begin{array}{c}\text { INHAL } \\
\text { SLOPE } \\
\text { PACTOR } \\
\text { (me/kg/day) }\end{array}$ \\
\hline $\begin{array}{l}\text { HEXAHYYDRO-1,3,5-TRINITRO-1,3,5- } \\
\text { TRIAZINE }\end{array}$ & $000121-82-4$ & $3 \mathrm{E}-3$ & .. & C & $1.1 \mathrm{E}-1$ & -. \\
\hline HEXANE, N & $000110 \cdot 54 \cdot 3$ & $\because$ & $2 \mathrm{E}-1$ & $-\cdot$ & $\cdots$ & 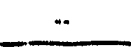 \\
\hline HEXAZINONE & $051235-04-2$ & 3.3E-2 & - & -- & $-\cdot$ & -. \\
\hline HYDRAZINE & $000302-01-2$ & 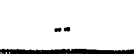 & - & $\mathrm{B2}$ & 3.0 & $-\cdot$ \\
\hline HYDROGEN CYANIDE & $000074-90-8$ & $2 \mathrm{E}-2$ & $\ldots$ & -- & -- & $-\cdot$ \\
\hline HYDROGEN CHLORIDE & $007647-01-0$ & - & $7 \mathrm{E}-3$ & - & - & $\cdots$ \\
\hline HYDROGEN SULFIDIE & $007783.06-4$ & $3 \mathrm{E}-3$ & $9 \mathrm{E}-4$ & - & 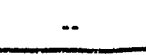 & 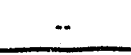 \\
\hline IMAZALIL & $035554-44-0$ & $1,3 \mathrm{E}-2$ & $\ddot{-}$ & - & $\cdots$ & .. \\
\hline IMAZAQUIN & $081335-37-7$ & $2.5 \mathrm{E}-1$ & - & - & $\because$ & $\cdots$ \\
\hline INDENO(1,2,3-CD)PYRENE & 000193.39 .5 & $\cdots$ & - & $\mathrm{B} 2$ & $\cdots$ & $\cdots$ \\
\hline IPRODIONE & $036734-19-7$ & $4 \mathrm{E}-2$ & $\ddot{-}$ & 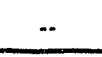 & -- & -. \\
\hline ISOBUTYL ALCOHOL & $0,00078-83-1$ & $3 E-1$ & -. & $-\cdot$ & -. & -. \\
\hline ISOPHORONE & $000078.59-1$ & $2 \mathrm{E} \cdot 1$ & - & C & $4.1 \mathrm{E} \cdot 3$ & $\cdots$ \\
\hline ISOPROPALIN & $033820-53-0$ & $1.5 \mathrm{E}-2$ & - & - & - & - \\
\hline $\begin{array}{l}\text { ISOPROPYL METHYL PHOSPHONIC } \\
\text { ACID }\end{array}$ & $001832.54-8$ & - & - & $\mathrm{D}$ & $\cdots$ & -. \\
\hline ISOXABEN & $082558.50-7$ & SE-2 & - & $\mathrm{C}$ & $\because$ & $\therefore$ \\
\hline KARATE (CYHALOTHIN) & $091465-08-6$ & $5 E-3$ & $\cdots$ & 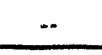 & .- & .- \\
\hline KERB $\quad$ (PRONAMIDE) & $023950-58.5$ & $7.5 \mathrm{E}-2$ & $\because$ & - & 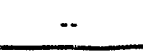 & 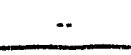 \\
\hline LACTOFEN & $077501-63-4$ & $2 \mathrm{E}-3$ & 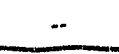 & $\cdots$ & $\cdots$ & $\cdots$ \\
\hline LEAD & $007439-92-1$ & - & $\because$ & $\mathrm{B} 2$ & -. & - \\
\hline LINURON & $000330-55-2$ & 2E-3 & - & C & $\cdots$ & - \\
\hline LONDAX & $083055-99-6$ & $2 E-1$ & - & 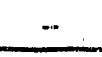 & $\therefore$ & $\cdots$ \\
\hline MALATHION & $000121-75.5$ & $2 \mathrm{E}-2$ & - & - & $\ddot{-}$ & $\because$ \\
\hline MALEIC ANHYDRIDE & $000108-31-6$ & $1 \mathrm{E}-1$ & - & $\ddot{-}$ & - & - \\
\hline MALEIC HYDRAZIDE & $000123-33-1$ & SE-1 & - & - & - & - \\
\hline MANEB & $012427-38-2$ & $5 E-3$ & $=$ & - & - & - \\
\hline MANGANESE & $007439.96-5$ & $1 \mathrm{E}-1$ & $4 \mathrm{E}-4$ & $\mathrm{D}$ & - & - \\
\hline MCPA & 000094.74 .6 & $5 E-4$ & - & $\ddot{-}$ & - & - \\
\hline MCPB & 000094.81 .5 & $1 \mathrm{E}-2$ & - & - & - & - \\
\hline MCPP & $000093-65-2$ & $1 \mathrm{E}-3$ & - & $\cdots$ & $\ddot{-}$ & - \\
\hline MEPIQUAT CHLORIDE & $024307.26-4$ & $3 E-2$ & - & - & - & $\ddot{-}$ \\
\hline MERCURY, INORGANIC & $007439.97-6$ & 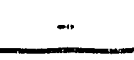 & - & D & $-\cdot$ & - \\
\hline MERPHOS & $000150-50-5$ & $3 \mathrm{E}-5$ & -- & - & $\cdots$ & - \\
\hline
\end{tabular}




\begin{tabular}{|c|c|c|c|c|c|c|}
\hline NAME & CASRN & $\begin{array}{c}\text { ORAL } \\
\text { RED } \\
(m g / z / d x y)\end{array}$ & $\begin{array}{c}\underset{\text { INHAi }}{\text { RPC }} \\
\text { (tog/at }(\mathrm{m})\end{array}$ & $\begin{array}{c}\text { EPA } \\
\text { GROUP }\end{array}$ & $\begin{array}{c}\text { ORAL } \\
\text { SLOPE } \\
\text { PACTOR } \\
(\text { (mgLE/dry) } \\
\end{array}$ & $\begin{array}{c}\text { INHAL } \\
\text { SLOPE } \\
\text { PACTOR } \\
\text { (mg/xg/day) } \\
\end{array}$ \\
\hline MERPHOS OXIDE & $000078-48-8$ & $3 \mathrm{E}-5$ & -- &.- & -. & 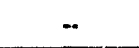 \\
\hline METALAXYL & $057837-19.1$ & $6 \mathrm{E}-2$ & $\ldots$ & - & -. &.- \\
\hline METHACR YLONITRILE & $000126-98-7$ & $1 \mathrm{E}-4$ & 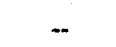 & -. & -- & -. \\
\hline METHAMIDOPHOS & $010265-92-6$ & SE-5 & - & -- & .. &.- \\
\hline METHANOL & $000067-56-1$ & SE-1 & - & .. & $-\cdot$ & -. \\
\hline METHIDATHION & $000950-37-8$ & $1 \mathrm{E}-3$ & -. & C & -. & - \\
\hline METHOMYL & $016752-77-5$ & 2.5E-2 & $-\cdot$ & - & - & $-\cdot$ \\
\hline METHOXYCHLOR & $000072.43 \times 5$ & $5 E-3$ & - & $\mathrm{D}$ & - & -. \\
\hline METHOXYETHANOL, $،$ - & $000109-86-4$ & -. & $2 E-2$ & -. & .. & - \\
\hline METHYL ACRYLATE & $000096-33-3$ & - & -. & $\mathrm{D}$ & - & $\ddot{-}$ \\
\hline METHYL E THYL KETONE & 000078.93 & -. & -. & $\mathrm{D}$ & $-\cdot$ & - \\
\hline METHYL ISOBUTYL KETONE & $000108-10-1$ & - & -- & - &.- & - \\
\hline METHYL MERCURY & $022967.92-6$ & $3 \mathrm{E}-4$ & - & - & -. &.- \\
\hline METHYL PARATHION & $000298-00-0$ & $2.5 E-4$ & - & - & - & - \\
\hline METHYL TERT-BUTYL ETHER & $001634-04-4$ & - & $5 E-1$ & -. & 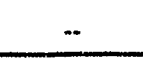 & - \\
\hline METHYLPHENOL 2- (O-CRESOL) & $000095-48-7$ & $5 \mathrm{E}-2$ & -- & C & -. & - \\
\hline METHYLPHENOL, 3- (M-CRESOL) & $000108-39-4$ & $5 \mathrm{E}-2$ & - & $\mathrm{C}$ & $\ddot{-}$ & $\ddot{-}$ \\
\hline METHYLPHENOL, 4- $\quad$ (P-CRESOL) & $000106-44.5$ & - & - & C & $\cdots$ & - \\
\hline $\begin{array}{l}\text { METHYLENE CHLORIDE } \\
\text { (DICHLOROMETHANE) }\end{array}$ & $000075-09-2$ & $6 \mathrm{E}-2$ & -. & B2 & $7.5 \mathrm{E}-3$ & -. \\
\hline $\begin{array}{l}\text { METHYLENE-BIS(N,N-DIMETHYL) } \\
\text { ANILINE, 4,4'- }\end{array}$ & $000101-61-1$ & - & - & B2 & $4.6 \mathrm{E}-2$ & - \\
\hline METOACHLOR & $051218-45-2$ & $1.5 \mathrm{E}-1$ & .. & C & 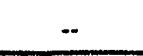 & - \\
\hline METRIBUZIN & $021087-64-9$ & 2.5E-2 & - & 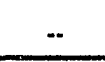 &.- & - \\
\hline MIREX & $002385-85-5$ & $2 \mathrm{E}-6$ & $\ddot{-}$ & - & - & - \\
\hline MOLINATE & $002212-67-1$ & $2 E-3$ & - & - & - & - \\
\hline NALED & $000300-76-5$ & $2 \mathrm{E}-3$ & - & - & -- & -. \\
\hline NAPHTHALENE & $000091-20.3$ & - & - & D & - & - \\
\hline NAPROPAMIDE & 015299.99 .7 & $1 \mathrm{E}-1$ & - & - & - & - \\
\hline NICKEL CARBONYL & 013463-39-3 & - & - & $\mathrm{B2}$ & - & $\cdots$ \\
\hline NICKEL SUBSULFIDE & $012035-72-2$ & - & - & $\mathbf{A}$ & - & - \\
\hline NICKEL (SOLUBLE SALTS) & $007440-02-0$ & $2 \mathrm{E}-2$ & - & $\cdots$ & - & - \\
\hline NICKEL REFINERY DUST & NO CASRN & - & -. & A & - & -. \\
\hline NITAPYRIN & 001929.82 .4 & $1.5 \mathrm{E}-3$ & - & $\cdots$ & .. & - \\
\hline NITRATE & $014797.55-8$ & $1.6 \mathrm{E}+0$ & $\cdots$ & - & .. & - \\
\hline
\end{tabular}




\begin{tabular}{|c|c|c|c|c|c|c|}
\hline NAME & CASRN & $\begin{array}{c}\text { ORAL } \\
\text { RDD } \\
\text { (mg/kg/day) }\end{array}$ & $\begin{array}{l}\text { INHAL } \\
\text { RrC } \\
\text { (mg/cu m) }\end{array}$ & $\begin{array}{c}\text { EPA } \\
\text { GROUP }\end{array}$ & $\begin{array}{c}\text { ORAL } \\
\text { SLOPE } \\
\text { FACTOR } \\
(\text { (mg/kg/dry })^{\prime \prime}\end{array}$ & $\begin{array}{l}\text { INHAL } \\
\text { SLOPE } \\
\text { PACTOR } \\
\text { (m\&kg/dry) }\end{array}$ \\
\hline NITRIC OXIDE & $010102-43-9$ & $1 \mathrm{E}-1$ & - & $\cdots$ & - & $\cdots$ \\
\hline NITRITE & $014797-65-0$ & $1 \mathrm{E}-1$ & 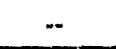 & - & $-\cdot$ & $\because$ \\
\hline NITROBENZENE & $000098-95-3$ & $5 E-4$ & 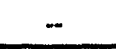 & $\mathrm{D}$ & - & - \\
\hline NITROGEN DIOXIDE & $010102-44-0$ & $1 \mathrm{E}+0$ & $\cdots$ & $\cdots$ & $\cdots$ & $\ddot{-}$ \\
\hline NITROGUANIDINE & $000556-88-7$ & $1 \mathrm{E}-1$ & - & D & $\cdots$ & $-\infty$ \\
\hline NITROPROPANE, 2 - & $000079-46-9$ & - & $2 \mathrm{E}-2$ & - & $\cdots$ & -- \\
\hline NITROSO-DI-N-PROPYLAMINE, N- & $000621-64-7$ & - & 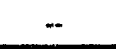 & $\mathrm{B2}$ & 7.0 & - \\
\hline NITORSO-DI-N-BUTYLAMINE; N- & $000924-16-3$ & - & 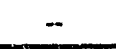 & B2 & $5.4 \mathrm{E}+0$ & -- \\
\hline NITROSODIETHANOLAMINE, N- & $001116-54-7$ & $\cdots$ & $\therefore$ & $\mathrm{B} 2$ & $2.8 E+0$ & $\therefore$ \\
\hline NITROSODIETHYLAMINE, N. & $000055-18-5$ & - & $\cdots$ & $\mathbf{B 2}$ & $1.50 \mathrm{E}+2$ & $\cdots$ \\
\hline NITROSODIMETHYLAMINE, N- & $000062-75-9$ & - & $\ddot{-}$ & B2 & $5.1 \mathrm{E}+1$ & 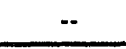 \\
\hline NITROSODIPHENYLAMINE, N & $000086-30-6$ & - & - & $\mathrm{B} 2$ & $4.9 \mathrm{E}-3$ & 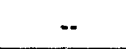 \\
\hline NITROSOMETHYLETHYLAMINE, N. & $010595-95-6$ & $\rightarrow$ & $\cdots$ & $\mathrm{B2}$ & $2.2 \mathrm{E}+1$ & $\ddot{-}$ \\
\hline NITROSOPYRROLIDINE, N- & $000930-55-2$ & - & - & $\mathrm{B2}$ & 2.1 & -- \\
\hline NONABROMODIPHENYL ETHER & $063936-56-1$ & - & $\cdots$ & D & $\because$ & $\cdots$ \\
\hline NORFLURAZON & $027314 \cdot 13-2$ & $4 \mathrm{E}-2$ & 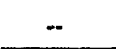 & $\cdots$ & $\cdots$ & - \\
\hline NUSTAR & $085509-19-9$ & $7 E-4$ & $\cdots$ & $\cdots$ & $\cdots$ & $\cdots$ \\
\hline OCTABROMODIPHENYL ETHER & $032536-52-0$ & 3E-3 & - & D & $\cdots$ & $\cdots$ \\
\hline $\begin{array}{l}\text { OCTAHYDRO-1,3,5,7-TETRANITRO- } \\
\text { 1,3,5,7-TETRAZOCINE }\end{array}$ & $002691-41-0$ & $5 E-2$ & $\cdots$ & D & $\cdots$ & $\cdots$ \\
\hline ORYZALIN & $019044-88-3$ & $5 E-2$ & - & $\mathrm{C}$ & $\cdots$ & $\cdots$ \\
\hline OXADLAZON & $019666-30-9$ & 5E-3 & - & $\cdots$ & $\cdots$ & $-\cdot$ \\
\hline OXAMYL & $023135-22-0$ & $2.5 \mathrm{E}-2$ & $\cdots$ & $\cdots$ & 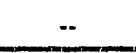 & $m$ \\
\hline PACLOBUTRAZOL & $076738-62-0$ & $1.3 \mathrm{E}-2$ & - & - & $\cdots$ & $\because$ \\
\hline PARAQUAT & $001910-42-5$ & $4.5 E-3$ & $\cdots$ & $\mathrm{C}$ & - & - \\
\hline PARATHION & $000056-38-2$ & - & - & C & -- & $\cdots$ \\
\hline PENDIMETHALIN & $040487-42-1$ & $4 \mathrm{E}-2$ & - & $\cdots$ & $\cdots$ & $\cdots$ \\
\hline PENTABROMODIPHENYL ETHER & $032534-81-2$ & $2 \mathrm{E}-3$ & $\cdots$ & D & $\cdots$ & $\cdots$ \\
\hline PENTACHLOROBENZENE & $0.00608-93-5$ & $8 E-4$ & $\cdots$ & - & 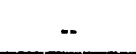 & -- \\
\hline PENTACHLOROCYCLOPENTADIENE & $025329-35-5$ & - & $\cdots$ & D & $\cdots$ & $\because$ \\
\hline PENTACHLORONITROBENZENE & $000082-68-8$ & $3 E-3$ & - & - & $\cdots$ & 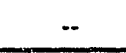 \\
\hline PENTACHLOROPHENOL & $000087-86-5$ & $3 \mathrm{E}-2$ & - & $\mathrm{B} 2$ & $1.2 \mathrm{E}-1$ & $\cdots$ \\
\hline PERMETHRIN & 052645.53 .1 & $5 \mathrm{E}-2$ & $\cdots$ & - & -- & - \\
\hline PHENANTHRENE & $000085-01-8$ & - & - & D & $\cdots$ & $\cdots$ \\
\hline
\end{tabular}




\begin{tabular}{|c|c|c|c|c|c|c|}
\hline NAME & CASRN & $\begin{array}{c}\text { ORAL } \\
\text { RRD } \\
(m \varepsilon / d / d y y)\end{array}$ & 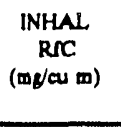 & $\begin{array}{c}\text { EPA } \\
\text { GROUP }\end{array}$ & $\begin{array}{c}\text { ORAL } \\
\text { SLOPE } \\
\text { PACTOR } \\
\text { (me/x/dry) })^{+1}\end{array}$ & $\begin{array}{c}\text { INHAL } \\
\text { SLOPE } \\
\text { PACTOR } \\
\text { (mexk/day)" }\end{array}$ \\
\hline PHENMEDIPHAM & $013684-63-4$ & $2.5 \mathrm{E}-1$ & - & - & - & - \\
\hline PHENOL & $000108-95-2$ & $6 \mathrm{E}-1$ & .. & D & -. &.. \\
\hline PHENYLENEDLAMINE, $M$ - & $000108-45-2$ & $6 \mathrm{E} \cdot 3$ & - & - & - & $\ddot{-}$ \\
\hline PHENYLMERCURIC ACETATE & $000062-38-4$ & 8E-5 & - & - & $\cdots$ & -. \\
\hline PHOSMET & $000732-11-6$ & $2 \mathrm{E}-2$ & - & 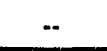 &. & -. \\
\hline PHOSPHINE & $007803-51-2$ & $3 E-4$ & - & -- & -- & 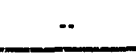 \\
\hline PHTHALIC ANHYDRIDE & $000085-44.9$ & $2 \mathrm{E}+0$ &.- & - & -- & -- \\
\hline PICLORAM & 001918-02-1 & $7 \mathrm{E}-2$ & -. & - & -- & $\therefore$ \\
\hline PIRIMIPHOS-METHYL & $029232.93-7$ & $1 \mathrm{E}-2$ & - & - & - & -. \\
\hline POLYCHLORINATED BIPHENYLS & $001336-36-3$ & - & -- & B2 & 7.7 & $-\cdot$ \\
\hline POTASSIUM CYANIDE & $000151-50-8$ & 5E-2 & 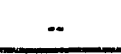 & - & - & 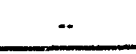 \\
\hline POTASSIUM SILVER CYANIDE & $000506-61-6$ & 2E-1 & - & - & - & .. \\
\hline PROCHLORAZ & $067747-09-5$ & 9E-3 & - & $\mathrm{C}$ & $1.5 \mathrm{E}-1$ & 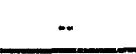 \\
\hline PROMETON & $001610-18-0$ & $1.5 \mathrm{E}-2$ & - & $\because$ & - & - \\
\hline PROMETRYN & $007287-19-6$ & $4 E-3$ & - & - & - & - \\
\hline PRONAMIDE (KERB) & $023950-58-5$ & $7.5 \mathrm{E}-2$ & - & $\ddot{-}$ & - & .. \\
\hline PROPACHLOR & $001918-16-7$ & $1.3 \mathrm{E}-2$ & - & - & $\cdots$ & $-\cdot$ \\
\hline PROPANIL & 000709.98 .8 & $5 \mathrm{E}-3$ & - & - & - & - \\
\hline PROPARGITE & $002312.35-8$ & $2 \mathrm{E}-2$ & - & - & - & - \\
\hline PROPARGYL ALCOHOL & 000107-19-7 & 2E-3 & - & - & - & -. \\
\hline PROPAZINE & $000139-40-2$ & $2 \mathrm{E}-2$ & - & - & - & - \\
\hline PROPHAM & $000122-42-9$ & $2 \mathrm{E}-2$ & - & - & - & - \\
\hline PROPICONAZOLE & $060207-90-1$ & $1.3 \mathrm{E}-2$ & - & - & - & - \\
\hline $\begin{array}{l}\text { PROPIONIC ACID, } 2(2,4,5 \\
\text { TRICHLOROPHENOXY)- }\end{array}$ & $000093-72-1$ & $8 \mathrm{E}-3$ & - & D & - & $-\cdots$ \\
\hline $\begin{array}{l}\text { PROPYLENE GLYCOL MONOMETHIYL } \\
\text { ETHER }\end{array}$ & $000107-98-2$ & - & 2 & -. & -. & -- \\
\hline PROPYLENE OXIDE & $000 \times 75-56-9$ & - & $3 \mathrm{E}-2$ & B2 & 2.4E-1 &.. \\
\hline PURSUIT & $081335-77-5$ & 2.5E-1 & - & - & -- & - \\
\hline PYDRIN & $051630-58-1$ & 2.5E-2 & - & - & - & -. \\
\hline PYRENE & $000129-00-0$ & $3 \mathrm{E}-2$ & - & $\mathrm{D}$ & 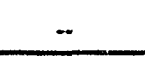 & - \\
\hline PYRIDINE & $000110-86-1$ & $1 \mathrm{E}-3$ & - & - & .. & $\ldots$ \\
\hline QUINALPHOS & $013593-03-8$ & $5 E-4$ & - & - & - & - \\
\hline RADIUM-228 & $015262-20-1$ & - & - & $\cdots$ & -. & - \\
\hline RADON-222 & $014859-67-7$ & - & - & - & 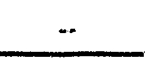 & - \\
\hline
\end{tabular}




\begin{tabular}{|c|c|c|c|c|c|c|}
\hline NAME & CASRN & $\begin{array}{l}\text { ORAL } \\
\text { RUD } \\
(m g / k y / d y)\end{array}$ & $\begin{array}{l}\text { INHAL } \\
\text { RSC } \\
\text { (mg/au m) }\end{array}$ & $\begin{array}{l}\text { PRA } \\
\text { GROUP }\end{array}$ & $\begin{array}{c}\text { ORAL } \\
\text { SLOPE } \\
\text { FACTOR } \\
(\text { [eg/Ke/dry })^{\prime \prime}\end{array}$ & $\begin{array}{c}\text { INHAL } \\
\text { SLOPE } \\
\text { PACTOR } \\
\text { (my/kg/day) }\end{array}$ \\
\hline RALLY (SYSTHANE) & $088671-89-0$ & 2.5E-2 & - & -- & - & 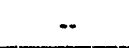 \\
\hline RESMETHRIN & $010453-86-8$ & $3 E-2$ & - & -- & $\cdots$ & .. \\
\hline ROTENONE & $000083-79-4$ & $4 \mathrm{E}-3$ & - & $\cdots$ & .. & .. \\
\hline SAVEY & $078587-05-0$ & $2.5 \mathrm{E}-2$ & - &.- & - & 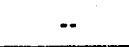 \\
\hline SELENIOUS ACID & $007783-00-8$ & $5 \mathrm{E}-3$ &.- & D & -. & -. \\
\hline SELENIUM AND COMPOUNDS & $007782-49-2$ & $5 E-3$ &.- & $D$ & $\cdots$ & - \\
\hline SELENUIM SULFIDE & $007446-34-6$ & -. & - & B2 & $\ldots$ & - \\
\hline SELENOUREA & $000630-10-4 \ldots$ & - & $\cdots$ & - & - & $\because$ \\
\hline SETHOXYDIM & $074051-80-2$ & $9 \mathrm{E}-2$ & -- &.- & $\cdots$ & -- \\
\hline SILVER & $007440-22-4$ & 5E-3 & -- & $\mathrm{D}$ & .. & -. \\
\hline SILVER CYANIDE & $000506-64.9$ & $1 \mathrm{E}-1$ & - & $\cdots$ & $-\cdot$ & $\cdots$ \\
\hline SIMAZINE & $000122-34-9$ & - & .. & $-\infty$ & $\cdots$ & - \\
\hline SODIUM DIETHYLDITHIOCARBAMATE & $000148-18-5$ & $3 \mathrm{E}-2$ &.- & $\ldots$ & - & 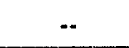 \\
\hline SODIUM FLUOROACETATE & $000062.74-8$ & 2E-5 & - & - & - & - \\
\hline SODIUM CYANIDE & $000143-33-9$ & $4 \mathrm{E}-2$ & - & - & - & - \\
\hline SODIUM AZIDE & $026628-22.8$ & 4E-3 & - & - & - & $\ldots$ \\
\hline SODIUM ACIFLUORFEN & $062476-59-9$ & $1.3 \mathrm{E}-2$ & - & -- & - & $\cdots$ \\
\hline STIROFOS (TETRACHLORVINPHOS) & $000961-11-5$ & $3 \mathrm{E}-2$ & .. & - & $\cdots$ & 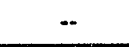 \\
\hline STRYCHNINE & $000057-24-9$ & $3 E-4$ &.- & - & $\cdots$ & $\cdots$ \\
\hline STTRENE & $000100-42-5$ & $2 \mathrm{E}-1$ & -. & $\cdots$ & $\cdots$ & -- \\
\hline TCMTB & $021564-17-0$ & - & - & -- & -- & .. \\
\hline TEBUTHIURON & $034014-18-1$ & 5E-2 & - & - & - & - \\
\hline TERBACIL & $005902-51-2$ & $1.3 \mathrm{E}-2$ & - & - & - & -- \\
\hline TERBUTRYN & $000886-50-0$ & $1 \mathrm{E}-3$ & - & - & - & - \\
\hline TETRABROMODIPHENYL OXIDE & $040088-47-9$ & - & $\ldots$ & $\mathrm{D}$ & - & - \\
\hline TETRACHLOROBENZENE, $1,2,4,5-$ & $000095-94-3$ & $3 E-4$ & - & - & $\ddot{-}$ & - \\
\hline TETRACHLOROCYCLOPENTADIENE & $000695-77-2$ & - & - & D & - & $\cdots$ \\
\hline TETRACHLOROETHANE, 1,1,1,2- & $000630-20-6$ & $3 E-2$ & $-\cdot$ & C & $2.6 \mathrm{E}-2$ & - \\
\hline TETRACHLOROETHANE, 1,1,2,2- & $000079-34-5$ & - &.- & C & $2.0 \mathrm{E}-1$ & -. \\
\hline TETRACHLOROETHYLENE, $1,1,2,2$ - & $000127-18-4$ & $1 \mathrm{E}-2$ & - & - & - & - \\
\hline TETRACHLOROPHENOL, 2,3,4,6. & $000058-90-2$ & $3 E-2$ & $\cdots$ & - & $\ddot{-}$ & $\cdots$ \\
\hline $\begin{array}{l}\text { TETRACHLOROTEREPHTHALIC ACID, } \\
2,3,5,6-, \text { DIMETHYL, ESTER (DACTHAL) }\end{array}$ & $001861-32-1$ & SE-1 & $-\cdot$ & -- & -- & -- \\
\hline TETRAETHYL LEAD & $000078-00-2$ & $1 \mathrm{E}-7$ & - & $\cdots$ & $\cdots$ & - \\
\hline
\end{tabular}




\begin{tabular}{|c|c|c|c|c|c|c|}
\hline NAME & CASRN & $\begin{array}{l}\text { ORNL } \\
\text { RRD } \\
(m y / k y / d x y)\end{array}$ & $\begin{array}{l}\text { INHAL } \\
\text { RRC } \\
(\mathrm{mg} / \mathrm{au} \mathrm{m})\end{array}$ & $\begin{array}{l}\text { EPA } \\
\text { OROUP }\end{array}$ & $\begin{array}{c}\text { ORAL } \\
\text { SLOPE } \\
\text { PACTOR } \\
(\text { (m\&/2/day) } \\
\end{array}$ & $\begin{array}{c}\text { INHAL } \\
\text { SLOPE } \\
\text { PACTOR } \\
\text { (me/te/day). }\end{array}$ \\
\hline TETRAETHYLDITHIOPYROPHOSPHATE & $003689.24-5$ & $5 \mathrm{E}-4$ & $\cdots$ & - & -. & - \\
\hline THALLIC OXIDE & $001314-32-5$ & - & -. & D &.- & -- \\
\hline THALLIUM (I) ACETATE & $000563-68-8$ & 9E-5 & -. & $\mathrm{D}$ & $\cdots$ & - \\
\hline THALLIUM (I) CARBONATE & 006533-73-9 & 8E-5 & $\cdots$ & $\mathrm{D}$ & -. & $m$ \\
\hline THALLIUM (I) CHLORIDE & $007791-12-0$ & $8 \mathrm{E}-5$ & - & D & - & - \\
\hline THALLIUM (I) NITRATE & $010102-45-1$ & 9E-5 & - & $\mathrm{D}$ & - & - \\
\hline THALLIUM SELENITE & $012039-52-0$ & 9E-5 & - & $D$ & - & - \\
\hline THALLIUM SULFATE & $007446-18-6$ & BE-5 & - & D & - & - \\
\hline THIOBENCARB & $028249.77-6$ & $1 \mathrm{E}-2$ & - & - & $\cdots$ & - \\
\hline THIOPHANATE-METHYL & $023564-05-8$ & $8 \mathrm{E} \cdot 2$ & -. & -. & $\cdots$ & - \\
\hline THIRAM & $000137.26-8$ & SE-3 & - & - & - & - \\
\hline TOLUENE & $000108-88-3$ & $2 \mathrm{E}-1$ & $\ldots$ & D & 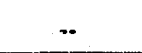 & $\cdots$ \\
\hline TOXAPHENE & $008001 \cdot 35-2$ & $\cdots$ & -. & B2 & 1.1 & - \\
\hline TRALOMETHRIN & $066841-25-6$ & $7.5 \mathrm{E}-3$ & - & - & - & - \\
\hline TRLALLATE & $002303-17-5$ & $1.3 \mathrm{E}-2$ & -. & $\ldots$ & - & - \\
\hline TRIASULFURON & $082097-50-5$ & $1 \mathrm{E}-2$ & - & .. & - & $-\cdot$ \\
\hline TRIBROMOBENZENE, 1,2,4- & $000615-54-3$ & 5E-3 & - & .. & - & -. \\
\hline TRIBROMOCHLOROMETHANE & $000594-15-0$ & - & - & D & - & -. \\
\hline TRIBROMODIPHENYL ETHER & $049690-94-0$ & - & - & D & $\ldots$ & -- \\
\hline TRIBUTYLTIN OXIDE & $000056-35-9$ & $3 \mathrm{E}-5$ & - & - & - & - \\
\hline $\begin{array}{l}\text { TRICHLORO-1,2,2- } \\
\text { TRIFLUOROETHANE,1,1,2- }\end{array}$ & $000076-13-1$ & $3 E+1$ & -- & - & -- & -- \\
\hline TRICHLOROBENZENE, 1,2,4- & $000120-82-1$ & - & - & D & - & - \\
\hline TRICHLOROCYCLOPENTADIENE & $077323-84-3$ & - & - & $\mathrm{D}$ & - & - \\
\hline TRICHLOROETHANE, 1,1,1- & $000071.55-6$ & - & - & $\mathrm{D}$ & - & - \\
\hline TRICHLOROETHANE, 1,1,2- & $000079-00-5$ & 4E-3 & - & $\mathrm{C}$ & 5.7E-2 & .. \\
\hline TRICHLOROETHYLENE & $000079-01-6$ & - & - & 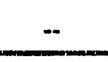 & -- & $\ddot{-}$ \\
\hline TRICHLOROFLUOROMETHANE & $000075-69-4$ & $3 \mathrm{E}-1$ &.- & - & - & -. \\
\hline TRICHLOROPHENOL, 2,4,5- & $000095-95-4$ & $1 \mathrm{E}-1$ & $\cdots$ & - & - & - \\
\hline TRICHLOROPHENOL, 2,4,6- & $000088-05-2$ & - & - & B2 & $1.1 \mathrm{E}-2$ & -- \\
\hline $\begin{array}{l}\text { TRICHLOROPHENOXYACETIC ACID, } \\
\text { 2,4,5- }\end{array}$ & $000093-76-5$ & $1 E-2$ & -. & -. & - & - \\
\hline TRICHLOROPROPANE, 1,1,2- & $000598-77.6$ & $5 E-3$ & - & - & - & $-\cdot$ \\
\hline TRICHLOROPROPANE, $1,2,3$ - & $000096-18-4$ & $6 \mathrm{E}-3$ & - & $\cdots$ & $m$ & - \\
\hline TRIDIPHANE & $058138-08.2$ & $3 \mathrm{E}-3$ & - & .. & - & -. \\
\hline
\end{tabular}




\begin{tabular}{|c|c|c|c|c|c|c|}
\hline NAME & CASRN & $\begin{array}{c}\text { ORAL } \\
\text { RRD } \\
(\operatorname{mg} / \mathrm{A} / \mathrm{d} y)\end{array}$ & $\begin{array}{c}\text { INHAL } \\
\text { RRC } \\
\text { (mg/al m) }\end{array}$ & $\begin{array}{c}\text { EPA } \\
\text { GROUP }\end{array}$ & $\begin{array}{c}\text { ORAL } \\
\text { SLOPE } \\
\text { PACTOR } \\
(\text { (E\&/tg/day })^{-1}\end{array}$ & $\begin{array}{c}\text { INHAL } \\
\text { SLOPE } \\
\text { PACTOR } \\
\text { (me/x/dry) }\end{array}$ \\
\hline TRIETHYLAMINE & $000121-44-8$ & - & $7 \mathrm{E}-3$ & $\ddot{-}$ & -. & 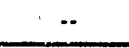 \\
\hline TRIFLURALIN & $001582.09-8$ & $7.5 \mathrm{E}-3$ & - & $\mathrm{C}$ & $7.7 \mathrm{E}-3$ & -- \\
\hline TRINITROBENZENE, 1,3,5- & $000099-35-4$ & $5 \mathrm{E} \cdot 5$ & - & - & -. & .. \\
\hline TRINITROTOLUENE, 2,4,6- & $000118-96-7$ & $5 E-4$ & $\cdots$ & C & $3.0 \mathrm{E}-2$ & - \\
\hline URANIUM, NATURAL & $007440-61-1$ & - & - & - & -- & .. \\
\hline URANIUM (SOLUBLE SALTS) & NO CASRN & $3 \mathrm{E}-3$ & - & - & .- & -. \\
\hline VANADIUM PENTOXIDE & $001314-62-1$ & $9 \mathrm{E}-3$ &.- & -- & - & -. \\
\hline VERNOLATE & 001929.77 .7 & 1E-3 & -- & -- & -. & -- \\
\hline VINCLOZOLIN & $050471-44-8$ & $2: \dot{E}-2$ & $\cdots$ & - & -. & -. \\
\hline VINYL ACETATE & $000108-05-4$ & - & $2 \mathrm{E}-1$ & - & -. & -- \\
\hline WARFARIN & $000081-81-2$ & $3 E-4$ & $\ldots$ & $\cdots$ & -. & -. \\
\hline WHITE PHOSPHORUS & $007723.14-0$ & $2 \mathrm{E}-5$ & - & $D$ & -. & -. \\
\hline XYLENE, MLXTURE & 001330.20 .7 & $2 \mathrm{E}+0$ & -- & $\mathrm{D}$ & - & .- \\
\hline ZINC AND COMPOUNDS & $007440.66-6$ & - & -. & $\mathrm{D}$ & - & - \\
\hline ZINC CYANIDE & $000557-21-1$ & 5E-2 & - & - & $\cdots$ & -. \\
\hline ZINC PHOSPHIDE & $001314-84-7$ & $3 E-4$ & - & -- & -. & $\therefore 1$ \\
\hline ZINEB & $012122-67-7$ & $5 \mathrm{E}-2$ & -- & -- & -. & .. \\
\hline
\end{tabular}

EPA CARCINOGENICITY GROUPS

\footnotetext{
$A=$ HUMAN CARCINOGEN

B1 = PROBABLE HUMAN CARCINOGEN

Limited evidence in human studies

B2 = PROBABLE HUMAN CARCINOGEN

Sufficient evidence from animal studies, inadequate evidence or no data from human studies

$C=$ POSSIBLE HUMAN CARCINOGEN

$D=$ NOT CLASSIFLABLE AS TO HUMAN CARCINOGENICITY
} 
ALPHURETICAL IMDEX TO STATUTES

IMDEX TO RU MABERS BY CFR CITATIOA

Januery/February 1992

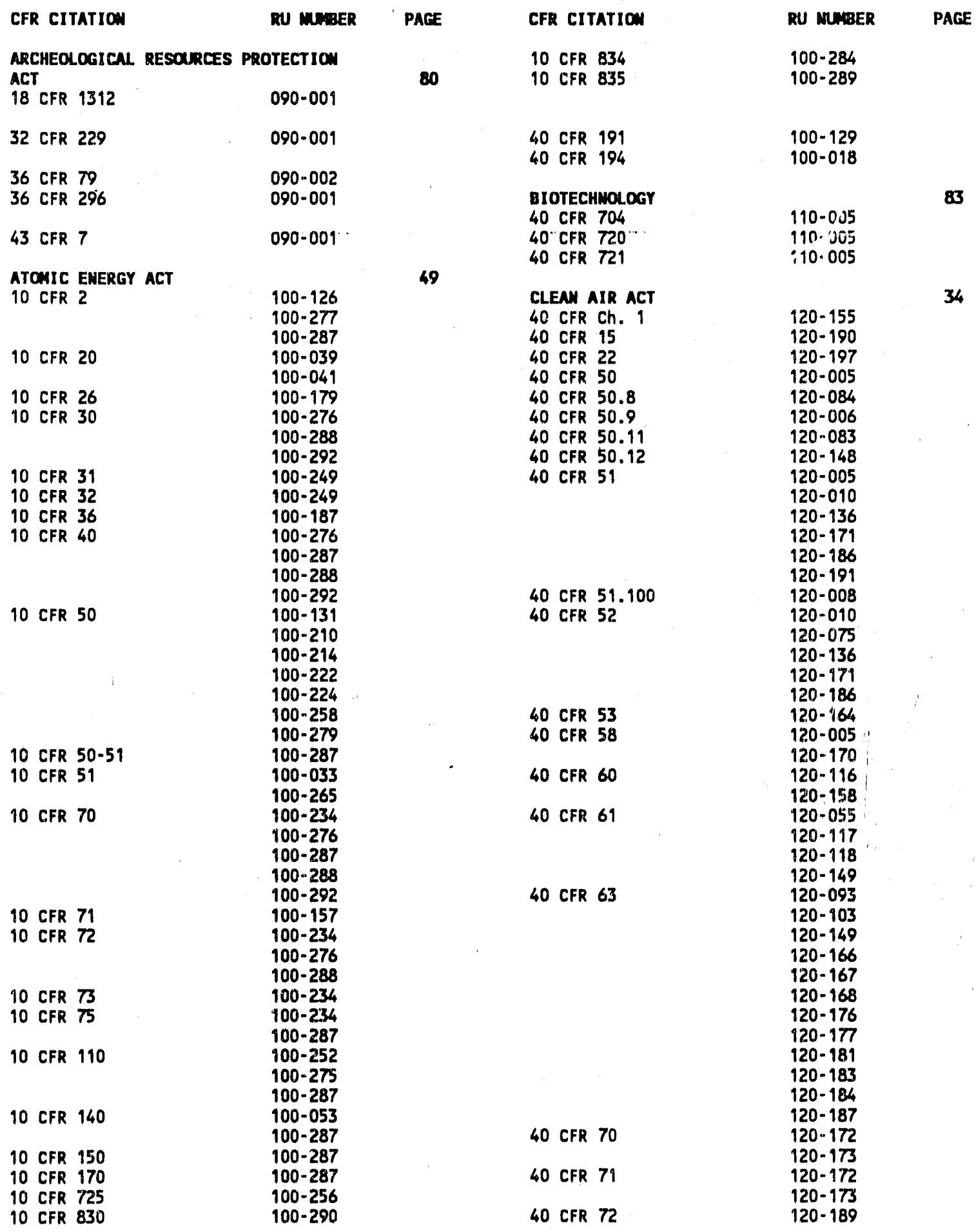




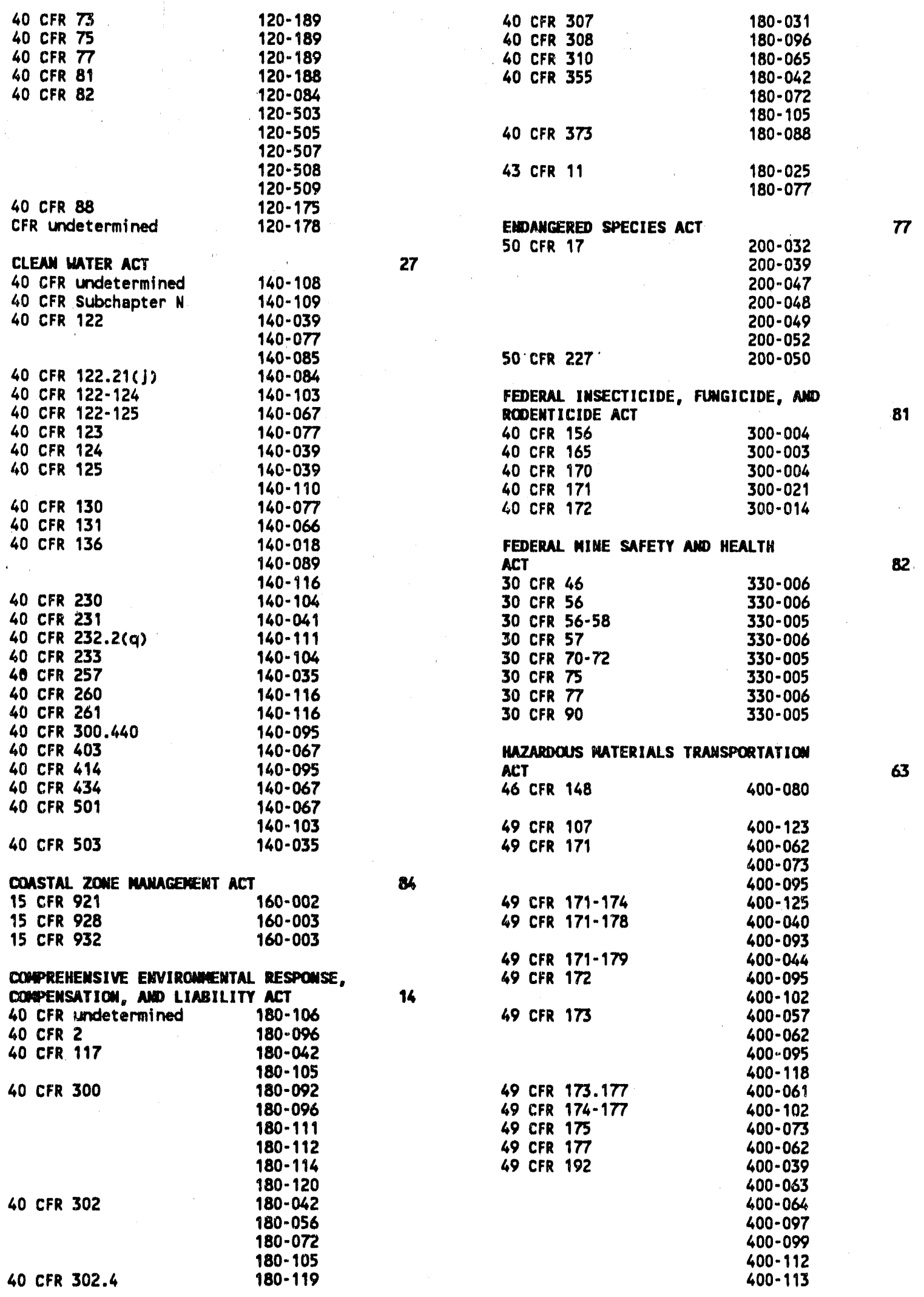




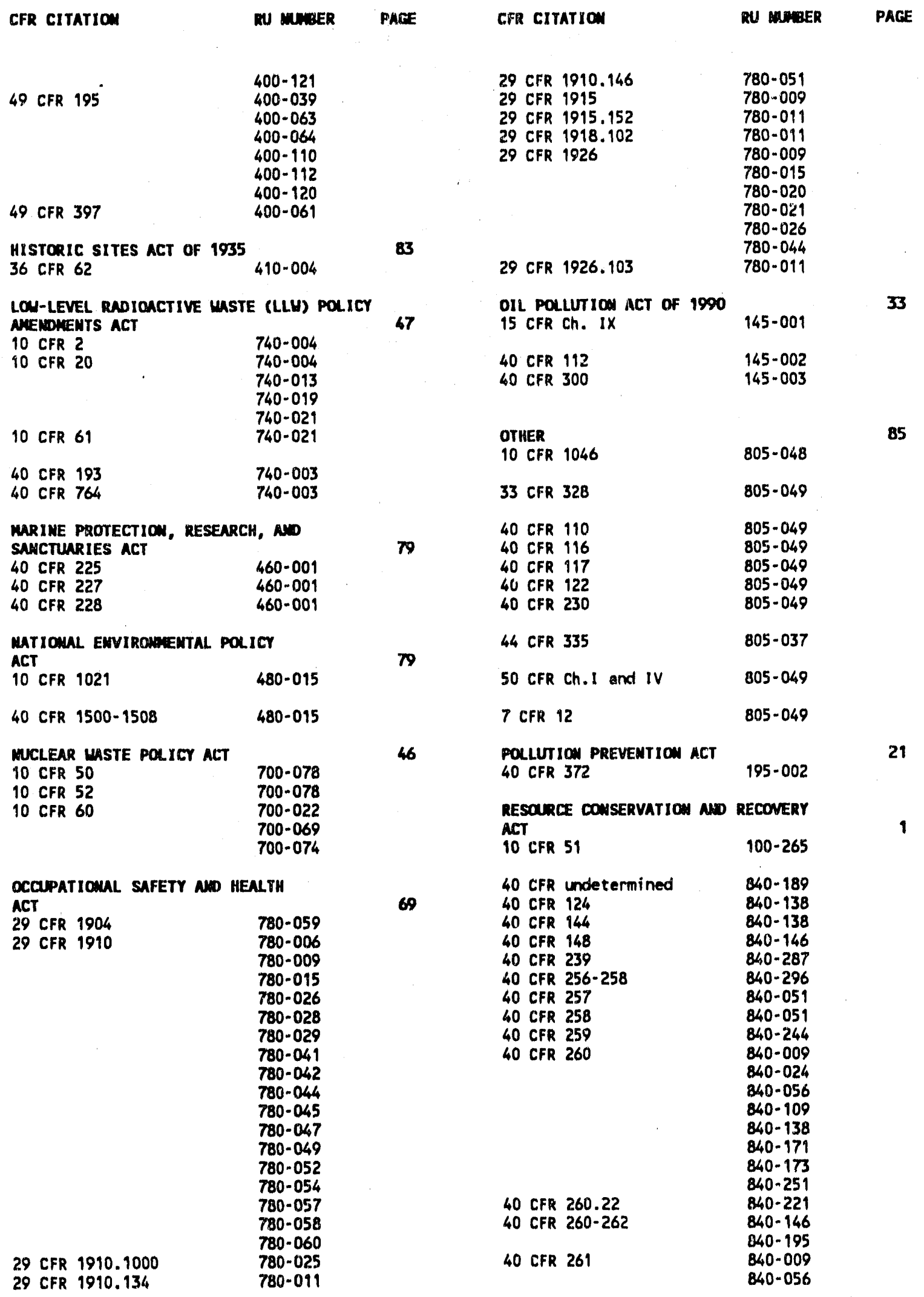


CFR Citation

40 CFR 262

40 CFR 262.49

40 CFR 264

40 CFR 264

40 CFR 264.75

40 CFR 264-265

40 CFR 265

40 CFR 266

40 CFR 268

40 CFR 270

40 CFR 270.41

40 CFR 270-271

40 CFR 271
DU MUmer

PACE

CFR CITATION

RU MUMER

PAGE

$840-107$

$840-173$

840-267

$840-268$

$840-288$

840-306

$840-307$

$840-173$

840.294

840.024

840-056

840-107

840-109

$840-131$

840-133

840-135

$840-138$

840-142

$840-148$

840-149

$840-171$

$840-173$

840.175

$840-176$

$840-248$

$840-251$

840-294

$840-146$

$840-195^{\circ}$

$840-024$

$840-056$

840-107

840-109

$840-133$

$840-135$

$840-138$

$840-142$

840-173

840-248

840-251

840-297

840-056

840-306

$840-307$

840-146

840-173

840-193

840-300

$840-305$

840.056

840.107

840-109

840-131

840-133

840-135

$840-138$

$840-149$

840-171

$840-173$

840-175

840-176

840-286

840-146

840-195

840-009

$\begin{array}{ll} & 840-024 \\ & 840-056 \\ & 840-109 \\ & 840-131 \\ & 840-133 \\ & 840-138 \\ & 840-179 \\ & 840-242 \\ & 840-267 \\ & 840-268 \\ & 840-295 \\ & 840-294 \\ & 840-151 \\ & 840-151 \\ 40 \text { CFR 271.10 } & 840-183 \\ 40 \text { CFR 271.15 } & 840-238 \\ 40 \text { CFR 271.16 } & 840-185 \\ 40 \text { CFR 272 } & 840-230 \\ & 840-248 \\ 40 \text { CFR 280 } & 840-230 \\ & 840-009 \\ 40 \text { CFR 281 } & 840-195 \\ 40 \text { CFR 302 } & 840-267 \\ & 840-268 \\ & 840-248\end{array}$

SAFE DRIMKIMG HATER ACT 40 CFR 35

22

40 CFR 141

$880-030$

880-007

880.010

$880-011$

$880-013$

880.034

$880-046$

$880-047$

$880-050$

880-053

$880-058$

40 CFR $142 \quad 880-007$

880.010

$880-011$

$880-013$

880-034

$880-047$

880-010

$880-013$

$880-027$

880.049

880-049

880-027

$880-049$

880-056

SUPERFUMD AREMDEEMTS AND REAUTHORIZATIOH ACT

$40 \mathrm{CFR}$ Ch. 1

190-014

$190-0.59$

40 CFR 28

40 CFR 35

190-014

190.014

190-042

40 CFR 305

40 CFR 355

190-056

190.051

190-053

190-060

40 CFR 373

190-038 
TOXIC SUUSTANCES CONTROL ACT

40 CFR Chapter 1

40 CFR 372

40 CFR 704

40 CFR 721

40 CFR 750

40 CFR 761

$920-126$

$190-061$

920.064

$920-124$

920-124

920.076

$920-119$

$920-125$

$920-132$

40 CFR 790

40 CFR 795

40 CFR 798

40 CFR 799

$920-053$

$920-123$

$920-130$

$920-113$

$920-123$

920-129

CFR undetermined
59

URANIUM MILL TAILINGS RADIATIOM CONTROL ACT

10 CFR 764

930.034

40 CFR 192

930-029

930.038 

INTERNAL DISTRIBUTION

1. Vince Adams

2. J. A. Shaakir-Ali

3. M. L. Ambrose

4. C. F. Baes

5. F. P. Baxter

6. B. A. Berven

7. T. J. Blasing

8. Gary Bodenste in

9. H. M. Braunstein

10. K. J. Brown

11. David Buhaly

12. E. H. Carroll

13. L. B. Cobb

14. Rick Collier

15. K. W. Cook

16. N. H. Cutshal

17. N. S. Dailey

18. J. A. Darling

19. D. L. Daugherty

20. J. M. Eaton

21. L. A. Eaton

22. G. K. Eddlemon

23. C. H. Estes

24. E. L. Etnier

25. Paula flowers

26. M. H. Francis

27. A. F. Fiedrick

28. K. V. Gabrielson

29. S. B. Garland

30. S. M. Glenn

31. C. D. Goins, Ir.

32. Carol Herdy

33. G. T. Hawkins

34. J. L. Haymore

35. C. L. Heckman

36. C. C. Hill

37. R. R. Hinton

38. S. K. Holladay

39. L. M. Houlberg

40. P. S. Hovatter

41. Wendy Hudson

42. Mallory Huxphreys

43. J. L. Johnson

44. R. L. Jolley

45. C. G. Jones

46. Dan Jones

47. T. A. Jones

48. R. A. Jump

49. R. H. Kingrea

50. D. C. Kocher

51. Francis Kovac:

52. Denton Lankford

53. B. D. Lawson

54. Allon Lewis

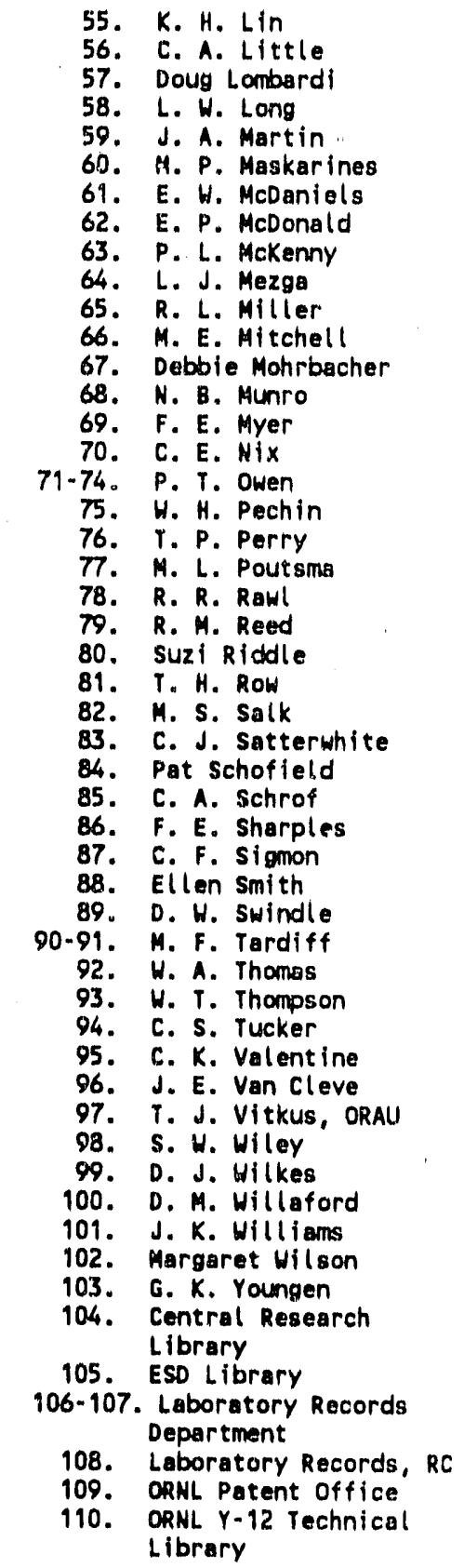

EXTERNAL OISTRIBUTION

111. David Abbott, EG\&G Mound Appl ied Technologies, P.0. Box 3000, Miamisburg, OH 45343

112. Marjorie Adans, Woodward-Clyde Federal services, \#1 Church St., suite 404, Rockville, MD 20850 
113. Pete Alexandro, NUS, 910 Clopper Road, P.0. Box 6032, Gaithersburg, MD 20877-0962

114. Major Len Alt, U.S. DOE, DP-641, Germantown, MO 20874

115. Stuart Altman, U.S. DOE, DP-644, GTN, Washington, DC 20545

116. Lauren B. Ames, Santa Fe Engineering, Ltd., 1900 Chamisa, Santa Fe, NM 87501

117. B. R. Anderson, B\&W Idaho Inc., 1580 Sawtelle: Box 1469, Idaho Falls, ID 83403

118. c. A. Anderson, Environmental Scientist, Idaho Operations Office, 785 DOE Place, MS \#1116, Idaho Falls, ID 83402

119. Lt. Col. Craig Anderson, HQ TAC/JAY, Langley AFB, VA 23665

120. Corl Angoli, U.S. Dept of Energy, Morgantown Energy Yech Ctr, P.O. Box 880, Morgantown,WV 26507-0880

121. Cassandra Anthony, SAIC, P.0. Box 2501, 800 Oak Ridge Turnpike, Oak Ridge, TN 37830

122. Major Dennis R. Armstrong, AFOEHL/RZ, Brooks Air Force Base, TX 78235-5501

123. Army Enviromental Office, Attn: ENVR-EP/Cabellon, Rm.1E6T7, Pentagon, Washington, OC $20310-2600$

124. Department of the Army, CETHA-EC-A/Bruce Pringel, U.S. Army Toxic \& Hazardous Materials Agency, Aberdeen Proving Ground, MD 21010-5401

125. Richard Artz, NOAA, Air Resources Lab, SSMCII, Rm. 9358, R/E/AR, 1325 East West Highway, Silver Springs, MD 20910

126. Irene Atney-Yurdin, Attorney, Brookhaven Area Office, 53 Bell Avenue, Upton, NY 11973

127. Andy Avel, U.S. Department of Energy, P.0. Box 398705, Cincinnati, OH 45239-8705

128. Jon E. Baer, Director, Landscape Architectural Service (088B4), Office of Facilities, Department of Veteran Affairs, 810 Vermont Avenue NH, Washington, DC 20420

129. James Bachmier, U.S. DOE, EH-232, GA-098, 1000 Independence Avenue, Washington, DC 20585

130. Charlotte Baker, Enviroment, Heal th, and Safety, University of California, Ervine, CA 92717

131. Lynn W. Ball, EG\&G Idaho, P.O. Box 1625, MS-1540, Idaho Falls, ID 83495

132. Steven N. Balone, H\&R Technical Associates (Weston Corp), 13126 Dairymaid Drive, Germantown, MO 20874

133. Ralph Barr, NUS Corporation, 910 clopper Road, P.0. Box 603), Fai thersburg, MD 20877-0962

134. Kris Barrett, Mitre CorP (HSO/YAQ), Brooks Air Force Base, TX 78235-5000

135. John H. Barry, U.S. DOE, Idaho Operations Office, 785 DOE Place, Idaho Falls, ID 83402

136. Pat Bartlett, ASO-463-8, FM Southern Region, 3400 Norman Berry Drive, P.O. Box 20636, East Point, a 30344

137. Lisa A. Bartsche, U.S. DOE, Princeton Area Office, P.O. 30x 102, Princeton, NJ 08542

138. John Bascietto, EH-231, GA-076, U.S. DOE, Forrestal Building, 1000 Independence Avenue, Washington DC 20585

139. John Baublitz, NE-20, E-420, U.S. Department of Energy, Washington, DC 20545

140. Paul A. Bean, U.S. DOE, EM-43, 1000 Independence Avenue, Washington, DC 20585-0002

141. Pat Bean, U.S. DOE, DP-534, GTH, Washington, DC 20585

142. C. 8. Becker, R3-28, P.O. Box 1970, Richland, WA 99352

143. Heino Beckert, U.S. DOE, EH-232, Forrestal Building, 1000 Independence Avenue, Washington, DC 20585 
144. Halter C. E. Beddoes, Defense Logistics Agency, DLA-S (RPDSO), Cameron Station, VA 22304

145. Henry Beiro, Earth Technology Corporation, 673 Emory Valley Road, Oak Ridge, TN 37830

146. Jerry Bell, Richland Operations Office, P.O. Box 550, Richland, WA 99352

147. Dr. John C. Belshe', Corps of Eng., DAEN-CWP-P, Rm. 7113, 20 Massachusetts Ave., N.W., Washington, DC 20314

148. Cathy Benediktsson, MML-465H, FMA Alaskan Region, 222 West 7th Avenue, \#14, Anchorage, AK 99513-7587

149. Paul J. Bemice, General Electric Company, Knolls Atomic Power Laboratory, P.0. Box 1072, Schenectady, NY 12301

150. S.J. Bensussen, Kaiser Engineers Hanford Company, P.O. Box 888, Richland, WA 99352

151. Chris Bergren, Hestinghouse Savennah River Company, Enviromental Protection Department, Building 742A, P.0. Box 616, Aiken, SC 29802

152. Raymond P. Berube, EH-20, 7A-075, U.S. Department of Energy, 1000 Independence Ave., Washington, DC 20585

153. Laura Beseris, 785 DOE Place, MS-1146, Idaho Falls, ID 83401-1562

154. Charles Billups, ER-8.2, U.S. Department of Energy, Washington, DC 20585

155. Frank Bingham, HPE/ECB, U.S. DOE, Nevada Operations Office, P.O. Box 98518, Las Vegas, NV 89193-8518

156. Walt Bird, Argonne Hational Laboratory, 9700 South Cass Avenue, ES\&H Division, Bldg. 201, Argonne, IL 60439

157. Warren Bodily, Kasier Engineering-Hanford, P.0. Box 888, Richland, WA 99352

158. Carol Boghosian, DOE/LBL Site Office, Mail Stop 508-3238, Lawrence Berkeley Laboratory, One Cyclotron Road, Berkeley, CA 94720

159. Carol Borgstrom, Director, Office of NEPA Oversight, U.S. Department of Energy, EH-25, Rm. 3E-080, 1000 Independence Ave., S.W., Hashington, DC 20585

160. Lucinda Borneman, Westinghouse Hanford CO., P.O. Box 1970, H4-57, Richland, WA 99352

161. A. M. Bosch, Faducah Gaseous Diffusion Plant, P.0. Box 1410, Paducah, KY 42001

162. C. Lee Bostic, NH Westinghouse Enviromental Center, 1234 Columbia Drive, Suite 203, Richland, WA $99352-4762$

163. Dave Breault, Knolls Atomic Power Lab, Kesselring Site Operations, 350 Atomic Project Road, Ballston Spe, NY 12020-2817

164. Major Tim Bridges, HO TAC/DEVP, Langley Air Force Base, VA 23665-5000

165. William A. Brobst, President, The Transport Environment, 6072 Currituck Road, Kitty Hawk, NC 27949

166. Robert T. Broili, MUS, 900 Trail Ridge Road, Aiken, SC 29801

167. D. E. Brown, Env. Protection Special ist, Kansas City Division, Dept 144-0829, Allied-Signal Corp, P.O. Box 419159, Kansas City, Mo 64141-6159

168. Tracy Brown, Battelle, Jackson Plaz8, Suite 300, 800 Oak Ridge Turnpike, Oak Ridge, TN 37831

169. J. E. Bruce, Rockwell-INEL, P.0. Box 1469, Mail Stop 2700, Idaho Falls, 1083403

170. Christopher A. Brudecki, Ogden Environmental \& Energy Services, 800 Oak Ridge Turnpike, Suite 103, Oak Ridge, TN 37830

171. Julie Brusslan, Advanced Sciences Inc., 2000 N. 15th St., Suite 407, Arlington, VA 22201 
172. Steve Buckley, Office of Qual i ty Assurance/Qual ity Control, U.S. DOE, EM-20, Rm 1G-066, 1000 Independence Ave., Washington, DC 20585

173. R. M. Burd, NUS Corporation, 900 Trail Ridge Road, Riken, SC 29801

174. Dr. Gordon Burley, U.S. EPA, ANR-458, 401 M St., S.W., Washington, DC 20460

175. Kenneth Burns, General Electric Company, 8152 Verbeck Drive, Manlius, WY 13104

176. C. Marshall Cain, Office of Federal Activities (A-104), U.S. EPA, 401 M street SW, Washington DC 20460

177. Sam Calhoun, TVA, P.O. Box 1010, NSE1D, Muscle Shoals, AL 35660-1010

178. Jahn Campisi, Advenced Sciences Inc., 6739 Acadermy, NE, Albuquerque, MM 87109

179. Doug Canete, Argonne National Laboratory, 370 L'Enfant Promenade, SW, suite 702, Hashington, DC 20024

180. Rita G. Carnes, Benchmark Envirommental Corp., 4501 Indian School Rd. NE, Suite 105, Albuquerque, NM 87110

181. Bob Carosino, Office of Chief Counsel, Richland Operations Office, MS-A452, P.O. Box 550, Richland, WA 99352

182. Jerry Carr, U.S. Department of Energy - WIPP, P.O. Box 3090, Carlsbad, NM 88221

183. John S. Cerrito, Knolls Atomic Power Laboratory, Kesselring Site Operation, 350 Atomic Project Road, Balliston Spa, NY 12020-2817

184. Charlie Chames, Aup-464-10, FaA Hestern Pacific Region, P.0. Box 92007, Worldway Postal Ctr., Los Angeles, CA 90009

185. Gloria E. Chavez, Sandia Hational Laboratories, Division 3222, P.0. Box 5800, Albuquerque, NM 87185

186. Chief, Environmental Sciences Branch, U.S. Department of Energy, 785 DOE Place, Idaho Falls, $1083402-4149$

187. Nathan A. Chipman, Westinghouse Idaho Nuclear Co., MS-3205, P.0. Box 4000, Idaho Falls, ID $83403-4000$

188. William D. Christensen, Ph.D., 100 Jefferson's Hundred, Williamsburg, VA 23185

189. Dr. A. L. Clark, HQUSAF/LEEVW, Bolling Air force Base; Washington, DC 20332-5000

190. Barbara Clark, Westinghouse - Savannah River, Building 703H, Aiken, SC 29802

191. Clifford E. Clark, Science Applications Corp., 1845 Terminal Drive, suite 202, Richland, WA 99352

192. Jennifer Clay, U.S. DOE, EM-20, Room 435, 12800 Middlebrook Rd., Germantown, MD 20874

193. L. Col. Robert C. egern, Execut ive Director, Armed Forces Test Management Board, Forest Glen station, WRAMC, Washington, DC 20307-5001

194. Jerry Coalgate, EH-231, GA-076, U.S. Department of Energy, 1000 Independence Ave., Washington, DC 20585

195. Joe Cochran, Sandia, Div-3223, Sandia National Labs, P.0. Box 5800, Albuquerque, MM $87185-5800$

196. Brenda Cole, ANL-W, P.O. Box 2528, MS-6000, Bldg. 752, Idaho Falls, ID 83403-2528

197. Alice Colston, Office of Management and Budget, NEOB, Room 8002, 17th Pennsylvania Ave., H.W., Washington, DC 20503

198. R. R. Colwell, Director of Maryland Biotechnology Institute, University of Maryland, Microbiology Bldg, College Park, MO 20742 
199. W. E. Cooper, Department of Zoology, College of Natural Sciences, Michigan State University, East Lansing, MI 48824

200. Ray Cooperstein, DP-131, 8-204/GTN, U.S. Department of Energy, Washington, DC 20545

201. Dr. Darrel Cornell, Environmental Management, OC-ALC/EMR, Tinker AFB, OK 73135

202. Debbie Craghill, P.0. Box 127, c/0 Naval Petroleum Reserves \#1, Tupman, CA 93276

203. Karin Crandall, NUS Corporation, 205-F Cody Lane, Aiken, SC 29803

204. Alan Crockett, EG\&G Idaho, P.O. Box 1625, Idaho Falls, ID 83415-2213

205. Martha Crosland, GC-11, 6A-113, U.S. Department of Energy, 1000 Independence Ave., Washington, DC 20585

206. June Crowe, IT Corporation, 312 Directors Drive, Knoxville, TN 37923

207. Debra Cully, Knolls Atomic Power Lab, P.0. Box 1072, Schenectady, WY 12301

208. Brian Culver, chief of Environmental Management, General Dynamics - EB Division, Kesselring site Field Office, P.0. Box 118 , Rock City Falls, NY 12863

209. Maureen Cunningham, SAIC, P.0. Box 2501, Oak Ridge, TN 37831

210. Rich Cunningham, U.S. DOE, DP-9, GE-063, 1000 Independence Avenue, Washington, DC 20585

211. Ruth Curley, KAPL, G1-101, P.0. Box 1072, Schenectady, NY 12301

212. Richard Dailey, EH-231, GA-076, U.S. Department of Energy, 1000 Independence Ave., Washington, DC 20585

213. Nancy E. Darling, Westinghouse Hanford Company, 420 Golf Club Road, suite 104, Lacey, WA 98503-1048

214. Abodul Q. Dasti, U.S. DOE, DP-222, GTN, Washington, DC 20545

215. Jerry Daub, Chen-Muclear Geotek, P.O. Box 14000, Grand Junction, CO 81502

216. James T. Davis, U.S. DOE, San Francisco Ops. Office, 1333 Broadway, Oakland, CA 94612

217. Defense Enviromental Restoration, 206 N. Washington Street, suite 100, Alexandrin, VA 22314

218. Hal Delaplane, FE-421, 3G-027, U.S. Department of Energy, 1000 Independence Ave., Washington, DC 20585

219. Cathy Demeroukas, U.S. Army, CERL/EAR, P.O. Box 9005, Champaign, IL 61826-9005

220. Willian Dennison, GC-11, DOE, Washington, DC 20545

221. James DePadro, Argonne National Laboratory, 9600 Cass Avenue, Argonne, IL 60439

222. Jack Depperschmidt, Mail Stop 1146, 785 DOE Place, Idaho Falls, ID 83402

223. Lata Desai, WIPP Technical Library, P.O. Box 2078, WIPP site 30 Miles SE of Carlsbad, Carlsbad, HM 88221

224. Linda J. Desell, RH-32, 7F-045, U.S. Department of Energy, 1000 Independence Ave., Washington, DC 20585

225. Leah Dever, EH-221, 36-092, U.S. Department of Energy, 1000 Independence Avenue, Hashington, DC 20585

226. Robin DeVore, Los Alamos National Laboratory, P.O. Box 1663, MS-J566, Los Alamos, NM 87545

227. Jerry DiCerbo, EH-231, GA-076, U.S. Department of Energy, 1000 Independence Ave., Hashington DC 20585

228. Steve Diedrich, AGL-436, FAM Great Lakes Region, O'Hare Lake Office Ctr., 2300 E. Devon Averue, Des Plaines, 1160018 
229. Jonathan Dion, DOE - RFP/MED, P.O. BOX 928, Golden, CO 80402

230. Director, U. S. Air Force Regional Compliance Office, 525 S. Griffin St., Box 116, Dallas, TK $75202-0516$

231. Ron Dixon, Legal Counsel, U.S, DOE, Planning, NPRC, P.0. Box 11, Tupman, CA 93276

232. DOE-RL, P.O. BOX 550, Richland, WA 99352

233. Dr. Willian W. Doerr, Progran Manager, Stone \& Hebster Environmental Corporation, P.0. Box 765, Boston, Ma 02102

234. David Dollins, U.S. DOE, Portsmouth Gaseous Diffusion Plant, P.0. Box 628, Piketon, OH 45661

235. Rick Donahoe, Bechtel Petroleum Operations, Inc., Naval Petroleum Reserves, P.0. Box 127, Tupman, CA 93276

236. Cathy Dufault, Naval Weapons Station Pier.X, NPrU, Charleston, SC 29408-7007

237. D. C. Durban, P.O. Box 1970, (N1-35), West inghouse Hanford Company, Richland, WA 99352

238. Ken Duvall, EH-232, U.S. Department of Energy, 1000 Independence Avenue, Washington, DC 20585

239. Emily Dyson, Roy F. Weston Company, 12800 Middlebrook Road, Suite 20\%, Germantown, MD 20874

240. Arnie Edelman, NE-47, Rm. F-442, GTN, U.S. Department of Energy, Washington, DC 20585

241. Robert Edwards, U.S. DOE, Paducah Gaseous Diffusion Plant, P.O. Box 1410, Paducah, KY 42001

242. Patricin A. Elker, US Army Material Command, ATTN: AMCSF-P, 5001 Eisenhower Avenue, Alexandria, VA 22333

243. Or. Donald R. Elle, EPS/EST, USDOE/NV, P.0. Box 98518, Las Vegas, NV 89193-8518

244. Dwight Emerson, Energetic inc., 7164 Gateway Drive, Columbia, MD 21046-2101

245. Dennis Engler, SAIC, 10770 Wateridge Circle, San Diego, CA 92121

246. Enviromental Compliance Division, OS/OASM/ECD/ H6319, U.S. Department of Commerce, Washington, DC 20230

247. Dr. Edward J. Evans, Jr., PMPMD, USAEHA, Aberdeen Proving Gds., N0 21010-5422

248. Tan Evans, DOE-HQ, EM-552, 12800 Middlebrook Road, Germantown, MD 20874

249. Gerry Fallon, Massachusetts Institute of Technology, Bates Linac, P.0. Box 95, Middleton, m 01949

250. William Fallon, Battelle, 370 L'Enfant Promenade, Suite 900, Mashington, DC 20024

259. Bill Farmer, EG\&G Mound Applied Technology, P.0. Box 3000, Miamisburg, OH 45343-3000

252. Ton Ferns, Westinghouse Hanford, P.O. Box 1970, MS H4-57, Richland, WA 99352

253. Marvin S. Fink, Safety and Health Manager, Room 6525, Main Building, U.S. Dept. of Justice, Washington, DC 20530

254. James J. Fiore, Director, Division of Facility and Site Decomissioning Projects (NE-23), U.S. Department of Energy, Washington, DC 20545

255. Ed Fisher, U.S. DOE, Morgantown Energy Tech. Ctr., P.0. Box 880, Morgantown, WW 26505

256. Joe Fitzgerald, EH-35, G-103, U.S. Department of Energy, 1000 Independence Avenue, Washington, DC 20585

257. N. J. Fix, EG G Idaho, Ine., P.0. Box 1625, MS-3414, Idaho falls, ID 83415

258. Roxanne flowers, DOE New Brunswick Lab, Building 350, 9800 South Cass Avenue, Argonne, IL $60439-4870$ 
259. Kenny Floyd, NIH, 9000 Rockville Pike, Bldg. 13, Rm. 2W64, Bethesda, MD 20892-00013

260. Brian Foley, DOE Field office - Richland Operations, P.0. Box 550, MS-A5-52, Richland, WA 99352

261. John Ford, DP-133, A-312, DOE, Washington, DC 20545

262. Mr. L.A. Fort, Westinghouse Hanford Co., P. O. Box 1970, R2-87, Richland, WA 99352

263. Willian Fortune, EH-231, DOE-HO, Forrestal Building, 1000 Independence Avenue, Washington, DC 2.0585

264. Micheel R. Fosdick, Vice President \& General Manager, John Brown E\&C Inc., 800 Werner Court, P.0. Box 90159, Casper, WY 82609

265. Thonas G. Frangos, Jacobs Engineering Group, P.0. Box 20008, Lakeland, FL 33806-2008

266. Dr. Clyde Frank, U.S. DOE, EM-50, 1000. Independence Ave., SW., Washington, DC 20585

267. Steve Frank, NP-50, U.S. DOE HQ, Forrestal Building, 1000 Independence Ave., Washington, DC 20585

268. J.F. Franklin, Bloedel Professor of Ecosystem Analysis, College of Forest Resources, University of Washington, Anderson Hall (AR-10), Seattle, WA 98195

269. Carl Gaddis, Pittsburgh Naval Reactors Office, P.0. Box 109, West Mifflin, PA 15122-0109

270. Glen R. Galen, Bechtel National, Inc., P.0. Box 350, Oak Ridge, TN 37831-0350

271. Jim Gemin, Wessinghouse-Hanford, P.O. Box 1970, Richland, WA 99352

272. George Garcia, Westinghouse-Savannah River, 37 Varden Drive, Aiken, SC 29803

273. Mike Garcia, U.S. Department of Energy, Albuquerque Ops.., Health Protection Division, P.O. Box 5400, Albuquerque, NM 87115

274. David Garoher, Westinghouse Materials Co. of Ohio, P.0. Box 353704, Mail Stop 31, Cincinnati, OH 45239-8704

275. Glenn Gardner, RW-42, 5A-05?, U.S. Department of Energy, 1000 Independence Avenue, Hashington, DC 20585

276. Dick Geiger, Chem-Nuclear Enviromental Services (A4-35), 825 Jaduin Avenue, P.0. Box 1786, Richland, HA 99352

277. David R. Gengozian, Environmental Quality Staff, SPB 25 214P, TVA, $400 \mathrm{~W}$. Summit Hill Drive, Knoxville, TN 37902-1499

278. Robert E. Giencke, General Electric/KAPL, P.O. Box 1072, MO G1-289, Schenectady, NY 12301

279. Martha Gitt, 265 Gladstone, Idaho Falls, 1083402

280. Chad Glem, U.S. Nuclear Reg. Commission, 1 White Flint North, 11555 Rockville Pike, MS-5E4, Rockville, Ho 20852

281. Melanie S. Goga, Halliburton NUS Environmental Corporation, Technical Library, Foster Plaza VII, Pittsburgh, PA 15220

282. Neal Goldenberg, EH-32, G-138, U.S. Department of Energy, Washington, DC 20545

283. Robert Gonzales, P.O. Box 1663, Los Alamos National Laboratory, MS-K489, HSE-13, L.os ALamos, NM 87545

284. Marjorie Gonzalex, L-39, Laurence Livermore Natl Lab, P.0. Box 808, Livermore, CA 94550

285. Scott Grace, U.S.DOE-Rocky Flats Office, Mail Stop EPA, P.0. Box 928, Golden, CO 80402-0928

286. Paul T. Grahovac, Westinghouse Idaho Nuclear Co., Inc., MS-3301, P.0. Box 4000, Idaho Falls, 1083403

287. Michael J. Green, MASA, 400 Maryland Ave. NH, Washington, DC 20546 
288. Steve Green, Manager, GOCO ES\&H Prograns, Westinghouse Electric Corporation, Gateway \#6 2185, 11 stanwix Street, Pittsburg, PA 15222

289. R. B. Greenstone (2AP 64C), Westinghouse-Bettis Laboratory, P.0. Box 79, West Mifflin, PA 15122

290. Charles Grua, U.S. DOE, NE-84, 1000 Independence Avenue, Washington, 20585

291. Rich Guida, NE-60, NR, 4N06, U.S. Department of Energy, Naval Reactors, Washington, DC 20585

292. Linda Guinn, EG\&G Idaho, P.0. Box 1625-3601, Idaho Falls, ID 83415

293. Cynthia B. Hall, Esq., Legal Organization 4000, Sandia National Laboratories, P.0. Box 5800, Al buquerque, NA 87185

294. Phoebe Hamill, DOE-Headquarters, FE-222, 1000 Independence Avenue, Washingt on, DC 20585

295. L. D. Hamilton, M.D. Phd., Head, Bionedical \& Envirommental Assessment Division, Brookhaven National Laboratory, Upton, Long Island, NY 11973

296. Kent Hancock, U.S. DOE, EM-352, Trevion 11, Rm. 374, Washington, DC 20585

297. James Hand, Corp of Eng., HODA-DAEN-CECH-PP, Pulaski Bldg, 20 Massachusetts Ave., N.W., Rm. 7113. Washington, DC 20314

298. Glen T. Hanson, 1628 Blair Court NE, Albuquerque, NM 87112

299. Ursula Harder, Manager, Environment, Heal th and Safety, General Electric Devices Dept., Mail Station-040, P.0. Box 2908, Largo, FL 34649

300. Timothy Harms, Office of Environmental Guidance and Compliance, EH-231, U.S. Department of Energy, 1000 Independence Ave., Washimaton, DC 20585

301. Ernest Marr, EM-20, 436 Trevion 11 Building, 12800 Middlebrook Rd., Germantown, MD 20874

302. Jim Martman, Environmental Manager, U.S. DoE, Western Areo Power Administration, P.O. Box 3700, Loveland, CO 80539-3003

303. Len Hayden, PEER Consultants, P.C., 575 Oak Ridge Turnpike, Oak Ridge, TN 37830

304. Don L. Hayes, Southwestern Power Adr, P.0. Box 1619, Tulsa, OK 74101

305. T. G. Hedahl, Manager, Enviromental Prograns, EG\&G Idaho, Inc., P.0. Box 1625, Idaho Fall 8,1083495

306. Poul L. Mendrickson, K6-63, Battelle - PNL, P.0. Box 999, Richland, WA 99352

307. Jim Heyser, 1900 Diamond Drive, Los Alamos, NM 87544

308. Mancy M. Hickok, 350 Atomic Project Road, Building 18, Room 101, West Milton, MY 12020

309. R. Douglas Mildebrand, U.S. Department of Energy, MS AS-SS, Box 550, Richland, WA 99352

310. Charles 1. Hochman, Office of Safety and Health, Department of Labor, 200 Const. Ave., N.W., Rm S22208, Washington, DC 2.0210

311. David Hoel, MP-53, U.S. Department of Energy, 1000 Independence Ave., Wash ington, DC 20585

312. Joseph Hoenscheid, COASO(E) 400 Army-Naw Drive, Suite "206, Arlington, VA 22202-2885

313. Jean Hoff, ICF, Inc., 9300 Lee Highway, Fairfax, VA 22031

314. Claire Holtzapple, DOE SAN/LDO, LLNL, Mail stop L-574, P.0. Box B08, Livermore, CA 94550

315. Dr. Kou-John Nong, Argonne National Laboratory, 9700 S. Cass Avenue, Argonne, IL, 60439

316. Pat Hoopes, U.S. Department of Energy, Kansas City Area Office, P.0. Box 410202, Kansas city, $19064141-0202$

317. David R. Hopkins, EPA - Federal Activities, 345 Courtland, Atlanta, GA 30365 
318. G.M. Hornberger, Professor, Department of Environmental Sciences, University of Virginia, Charlottesville, VA 22903

319. HO AFESC/RDV, Stop 21, Tyndall Air Force Base, FL 32403

320. Linda M. Hubbard, Bechtel National, Inc., P.0. Box 350, Oak Ridge, TN 37831-0350

321. J. H. Huckabee, Manager, Ecological Studies Program, Electric Power Research Institute, 3412 Hillview Avenue, P.O. Box 10492, Palo Alto, CA 94303

322. Joseph Hugo, Chief, Pollution Control Section, NIH, Bldg. 13, Room 2H64, Bethesda, MD 20892 (disk copy only)

323. Jerry Humman, EH-32, U.S. DOE, GTN, Washington, DC 20545

324. David P. Hutchison, EGsG Ideho, P.0. Box 1625, MS-2411, Idaho Falls, ID 83415

325. Leonord Hutterman, HIMCO, Box.4000-3906,. Idaho. Falls, ID 83403

326. T. B. Hyde, U.S. Dept of Energy, Albuquerque Operations Office, Management Support Div., Albuquerque, NM 87115

327. David S. Ingle, Safety and Occupational Heat th Manager, U.S. Department of Energy, Pinellas Area office, P.0. Box 2900, Largo, FL 34294

328. Inholation Toxicology Research Institute, Health Protection, P.0. Box 5890, Albuquerque, NM 87115

329-331. John Jaacks, c/O SAIC, MS 2-5-1, 1710 Goodridge Drive, MCLean, VA 22102

332. Jim Jackson, EGRG Idaho, P.0. Box 1625, Mail stop 3414, Idaho Falls, I0 83415-3414

333. Robert R. Jackson, Science Applications, 1845 Terminal Drive, Richland, WA 99337

334. Vernon 2. Jakubowski, 18-101, Knolls Atomic Power Laboratory, 350 Atomic Project Road, Ballston Spa, WY 12020-2817

335. Mr. Charles F. James, M-471, Dept. of Transportation, 4007 th St, S.W., Rm. 2308, Washington, DC 20590

336. Richard Janowiecki, EG\&G Mound Applied Technologies, P.0. Box 3000, Miamisburg, OH 45343-0987

337. C. Jierree, U.S. Department of Energy, Rocky Flats Area Office, P.0. Box 928, Golden, Co 80401

338. Janet Johnson, IMEL, 1955 Freemont Averuve, P.0. Box 4000, Idaho Falls, I0 83403-4000

339. Teresa G. Jordan (Attn: BLD. 742-A), Westinghouse - Savannah River Company, P.O. BOx 616, Aiken, SC 29802

340. George Y. Jordy, Director, Office of Progran Analysis, Office of Energy Research, ER-30, G226, U.S. Deparement of Energy, Washington, DC $20545^{\circ}$

341. Reed Kaldor, Advanced Sciences, Inc., 1T77 Terminal Drive, Richland, WA 99352

342. Mark Kamiya, Argonne Mational Laboratory, ES\&H Division, 9700 S. Cass Avenue, Argonne, IL 60439

343. Col. Don Kare, Env Division (AF/LEEY), Bolling AFB, Washington, DC 20332-5000

344. George Kaplan, ME-70, Rm. A-423, U.S. DOE, GTN, Washington, DC 20585

345. Allison V. Keebler, ERCE, 800 Oak Ridge Turnpike, Suite 103, Oak Ridge, TN 37830

346. E. Kehoe, SSgt, USAF NCCIC, Environmental Law Division, Office of the Judge Advocate General, AF/JACE, Bolling AFB, DC 20332-6128

347. R. R. Kemps, Director, Office of Project and Facilities Manegement, 1000 Independence Ave., 5C-032/forrestal Bldg, Washington, DC 20585 
348. J.C. Kent, Westinghouse Idaho Nuclear Co., Inc., P.0. Box 4000, Mail stop 5209, Idaho Falls, ID 83403

349. Philip H. Kier, Energy and Envirommental systems Division, Bldg. 362, Argonne National Laboratory, 9700 S. Cass Ave., Argonne, IL 60439

350. James C. Killen, U.S. DOE, Planning, HPRC, P.O. BoX 11, TUpman, CA 93276

351. Howard Kimpton, ACM-440, FM Technical Center, Atlantic City Airport, NJ 08405

352. Anthony King, Rosne State Waste Management Training Center, 728 Emory Valley Rosd, Oak Ridge, TN 37830

353. Kay King, Theta Technologies, $101 \mathrm{E}$. Tennessee Ave., Oak Ridge, TH 37830

354. Tim Kirkpatrick, EG\&G Idaho, MS-2411, P.0. Box 1625, Idaho Falis, ID 83415

355. Alan D. Kirsch, EG\&G Idaho, P.0. Box 1625-3601, Idaho Falls, ID 834,15

356. Mike Kleinrock, EM-20, 8H-050, U.S. DOE, Forrestal Building, 1000 Independence Avenue, Washington, DC 20585

357. Margret A. Knech, EG\&G Idaho, P.O. Box 1625, Idaho Falls, ID 83415-2212

358. J. P. Knight, Director, Office of Nuclear Safety, EH-33, G-107, U.S. Department of Energy, Washington, DC 20545

359. Ronald Knisley, MA-202.3, 4F-066, U.S. Departmerit of Energy, 1000 Independence Ave., Washington, DC 20585

360. Terri Knudsen, Rockwell International, Rocky Flats Plant, P.0. Box 464, T-130B, Golden, CO 80402-0464

361. Duane A. Krudson, ANL, 9700 s. Cass Avenue, 203-ER, Argonne, IL 60439

362. Edward J. Kochem, General Electric, Bldg. F3-7, P.0. 80x 1072, Schenectady, NY 12301

363. Ronald Kolzow, E00-201, 9700 south Cass Avenue, Argonne National Laboratory, Argonne, IL 60439

364. James Koonce, Lawrence Berkeley Laboraçory, MS 50A-5104, Oakland, CA 94720

365. Ted Koss, U.S. DOE, EH-232, 1000 Independence Avenue, Washington, DC 20585

366. George E. Krauter, Stone \& Webster, 702 S. Ill inois Avenue, Suite B-104, Oak Ridge, TN 37830

367. Mike Krupinsky, Mansgement Analysis Co., 12671 High Bluff Drive, San Diego, CA 92130

368. Or. Art Kubo, BDM International, 7915 Jones Branch Drive, McLean, VA 22102

369. Deborah Kufen, EG\&G Idaho, Inc., P.0. Box 1625, Idaho Falls, I0 83415-3505

370. Major Marcia Kurtz, 1606 ABH/JA, Kirtland AFB, MM 87117-5000

371. Tapio Kuusingn, (K6-55), Pacific Worthwest Laboratory, P.O. Box 999, Richland, HA 99352

372. Susan Kuznick, GC-31, 68-256, U.S. Department of Energy, 1000 Independence Avenue, Washington, DC 20585

373. Robert T. Lackey, Deputy Director, EPA Environmental Research Lab., 200 SW 35 th Street, Corvall is, OR 97333

374. A. G. Ladino, U.S. Department of Energy, Anarill lo Area Office, P.0. Box 30030, Amarillo, TX 79120

375. Mr. Phil Lami, Director, Enviromental Planning Division, Air force Regional Civil Engineer-Western Region, AFRCE-WR/ROV, 630 Sansome St., Room 1316, San Francisco, CA $94111-2278$

376. P. S. Lanthan, Manager of Environmental Special Project8, Chem-Nuclear Geotek, P.0. 80x 1400 , Grand Junction, CO 81502.5504 
377. Fred N. Larsen, Allied-Signal Inc.. P.0. Box 1159, Department 837-XD43, Kansas Clty, MO 64141

378. JaY Larson, CIH, U.S. DOE (ER-8.1), 19901 Germantown, MD 20874

379. BIll Lattin, U.S. DOE, Idaho Operations Office, t'85 DOE Place, Idaho Falls, ID 83402

380. J. Thomas Lawell, Colonel, USAF, Director, Envirchmental Management, SM-ALC/EM, McClellan AFB, CA $95652-5990$

381. Andy Lawrence, EH-231, GA-076, U.S. DOE, 1000 Inclependence Ave., Washington, DC 20585

382. Kurt Lawton, Martin Marietta Energy Systens, Box 628, M.S. 3209, Piketon, OH 45661

383. Eugene Lehr, P-14, Department of Transportation, 4007 th St, S.W., Rm. 9217, Washington, DC 20590

384. John Lehr, .DP-124, A-220, U.S. Department of Energy, Washington, DC 20545

385. Kathleen Leonard, Westinghouse Hanford, P.0. Box 1970, 82-19, Richland, WA 99352

386. Mary Lou Leonard, 3021 San Patricia NW, Albuquerque, NM 87107

387. Ann M. Lesperance, MSIN K6-58, P.0. Box 999, Richland, WA 99352

388. Nick Lestenkof, ANM-463.H, Federal Aviation Administration, 1601 Lind Avenue SW, Renton, WA $98055-4056$

389. Martin J. Letnurneau, U.S. Department of Energy, EM-22, Trevion 11, Washington, DC 205850002

390. Donna M. Leydorf, SAIC, Division 380, P.0. Box 2501, Oak Ridge, IN 37831

391. Library, Bechtel National Inc., Box 350, Oak Ridgr, TN 37831

392. Library, U.S. Enviromental Protection Agency Region IV, 345 Courtlard Street, Atlanta, GA $30365-2401$

393. Library, Weston, Inc., 955 L'Enfant Plaza, SW, suite 800, Washington, DC 20024

394. Edward Liebsch, HDR Engineering, Inc., 5401 Gamble Drive, Suite 300, Minneapol is, MN 554161518

395. G. E. Likens, Director, The New York Botanical Garden, Institute of Ecosystem Studies, The Mary flagler Cary Arboretum, Box AB, Millbrook, NY 12545

396. Jim Littlejohn, Nevada Operations Office, U.S. DOE, P.0. Box 98518, Las Vegas, NV 89193-8518

397. Chuck Ljungberg, U.S. DoE, West Valley Project office, P.0. Box 191, West Valley, NY 14171

398. Jim B. Lloyd, Southeostern Power Adn, Semul Elbert Building, Elberton, aA 30635

399. Mary Alice Locke, Federal Aviation Administration, (AEE"20), 800 Independence Avenue SW., Washington, DC 20591

400. Jim Long, MAC-51, FAM Aeronautical Center, 6500 s. MacArthur, P.0. Box 25082, Oklahoma City, ok 73125

401. Jeffrey d. Longaker, SAIC, P.O. Box 2501, 800 Oak Ridge Turnpike, Oak Ridge, TN 37831

402. Susan W. Longley, Sandia National Laboratories, P.O. Box 5800, Division 3222, Albuquerque, NM 87185

403. Jackit Loop, EGSG Idaho, P.0. Box 1625, Idaho Falls, ID 83475-2300

404. Margaret LOU, U.S. DOE, Bartlesville Project Office, P.0. Box 1398, Bartlesville, OK 74003

405. James O. LOW, U.S. DOE, Albuquerque Operations Office/SPD, P.O. Box 5400, Albuquerque, NM 87115 
406. Richard P. Lubert, General Electric, Knoll Atomic Power Laboratory, P.0. Box 1072, Schenectady, WY 12301

407. Karan Lymcoln, P.0. Box 919, Los Alamos, NM 87544

408. John J. Lyons, U.S. Department of Energy, Duyton Area Offlce, P.0. Box 66, Miamiaburg, OH 45342

409. Steven Maheras; EGrG Idaho, P.0. Box 1625, Idaho Falls, I0 83415

410. Dole R. Mahnke, MUES, P.0. Box 29304, Dept. 3162, New Orleans, LA 70189

411. Margaret Makram, U.S. DOE, Los Alamos Area Office, 52835 th St., Los Alamos, NM 87544

412. J. Malanchak, office of Enviromental Process and Effects Research, U.S. Environmental Protection Agency, 401 M street, SW, RD-680, Washington, DC 20460

413-414. James Mangeno, (NE-60), U.S. Dopartment of Energy, P.O. Box 23865, Washington, DC 20585

415. C. J. Mankin, Director, Oklahoma Geological Survey, Energy Center, $100 \mathrm{E}$. Boyd, Rm N-131, Norman, OK 73019-0628

416. Diane Mann, EN, U.S. Army Const. Res Lab., P.0. Box 4005, Champaign, IL 61820

417. Lois Marik, ESS Division, DOE San Francisco Field Office, 1333 Broadway, Oakland, CA 94612

418. Marty L. Martin, P.0. Box 1970, MSIN XO-35, Richland, WA 99352

419. Stan Massey, ACE-462, FMA Central Region, 601 East 12 th Street, Federal Blang., Kansas City, MO 64106

420. Ken Hathias, A0420, Western Area Power Adm, P.O. Box 3402, Golden, Co 80401

421. John Mathur, DP.123, A-227, U.S. Department of Energy, Washington, DC 20545

422. M. L. Matthews, U.S. Dept of Energy, Uranium Mill Tailings Project Offlice, P.0. Box 5400, Albuquerque, MM 87115

423. A. J. Matule, Westinghouse Idaho Nuclear Co., Inc., P.0. Box 4000, Maill stop 5209, Idaho Falls, 1083403

424. Carl A. Mazzola, Stone Webster, South Operations, Woodside Executive Office Park, suite F-25, 1359 silver Bluff Road, Aiken, SC 29803

425. David R. Mccallister, Laurence-Allizon Division, John Brown ERC Inc., MPR HB Field Office, P.0. Box 90159, Casper, WY 82609

426. Virginia AcCauloy, Manager, Operations Program, General Electric Devices Dept., Mail Station-009, P.0. Box 2908, Largo, FL 34649

427. Doug MCCOY, TDEC, Director of DOE Oversight, 761 Emory Valley Road, Oak Ridge, TN 37830

428. Beth McDougald, Lee Wan 2 Associates, Infurmation Resource Center, 105 Broadway, Oak Ridge, TN 37830

429. Jim McGovern, AEA-462, FM Eastern Region, JFK International Airport, Fitzgerald federal Blong., Jamaica, NY 11430

430. Susan M. Megowan, Arthur D. Little, Inc., Acorn Park, Cambridge, MA 02140

431. David C. Mcoraw, Lawrence Berkeley Laboratory, Mail stop 90-1106, 1 Cyclotron Road, Berkeley, CA 94720

432. K. P. McKay, Bettis Laboratory, APOBE, P.O. Box 79 Weat Mifflin, PA 15122-0070

433. Jesse McKendree, EGsG Mound Appl led Technologies, P.O. Box 3000, Miamisberg, OH 45343-3000

434. Sylvia M. Medina, EasG Idaho, Ine., P.0. Box 1625, Idaho Falls, 1083415

435. J. A. Mewhinney, U.S. Dept of Energy, Waste Isolation Pilot Plant Project Office, P.0. Box 3090, Carlsbad, NM 88221. 
436. Raymond W. Mlahals Uaker and Assoctates, Inc., 800 Commerce Rd., Wast " sulte 500, New Orleans, LA 70123

437. Mark Mlkatauage, Chief, OHB/SO/ONs, Central Intelligence Agency, Washington, DC 20505

438. Caryle B. MIller, NP.52, U.S. DOE, 1000 Independence Avenue, SW, Washington, DC 20585

439. J. F. Mlller, Director, Enviromental Planning Division, Office of the Assistant Secretary for Commenlty Planning and Development, U.S. Department of Housing and Urban Development, Washington, DC 20410-7000

440. Steve Miller, GC-11, U.S. DOE, 1000 Independence Avenue SW, Washington, DC 20585

441. Stephen V. Mitz, SAIC, P.O. Box 2501, Oak Ridge, TN 37831

442. Jaffer Mohiluddin, BDM International, 12850 Middlobrook Road, Germantown, MD 20874

443. Darlo Monte, EH-10, OB 096-A, U.S. DOE, 1000 Indefendence Avenue, Washington, DC 20585

444. Kenneth S. Moor, Idaho National Engineering Lab, P.0. Box 162.5, Idaho Falls, 10 83415-2213

445. Herman Moore, U.S. Dot:, Wost Valley Prolect Offlce, 10300 Rock spring Road, West Valley, NY 14171

446. Scott Moore, 800 Oak Ridge Turnpike, Jackson Tower, suite C-102, Oak Ridge, TN 37830

447. Anthony R. Morrell, Assistant to the Acministratol for Enviroment, Bonneville Power Administration, P.0. Box 3621, Ad, Portland, OR 97208

448. Kristine Morris, DPn 10, 4A-043, U.S. Department of Energy, 1000 Independerice Ave., Washington, DC 20585

449. Robert Muhly, U.S. Army Toxic and Hazardous Materials Agency, Environmental and Health office, Bldg 4406EA, Aberdeen Proving Oround, MD 21010

450. Harriet Mulvany, Traller T-130C, EGsO Rocky Flats, P.0. Box 464, Golden, C0 80402-0464

451. Herbert Myers, CE-62, 6B-070, U.S. Department of Energy, 1000 Independence Avenue, Washington, DC 20585

452. Katherime Nakata, U.S. DOE, EH-231, Forrestal Building, 1000 independence Avenue, Washington, DC 20585

453. Mark Neblett, Naval Sea Systens Command (08U), Department of Navy, Washington, DC 20362-5101

454. Joan K. Neff, IMEL, 1955 fremont Avenuse, P.0. Box 4000, Idaho Falls, 10 83403-4000

455. Jill Newby, SAIC, 2765 South Highland Drive, suite 101, Las Vegas, NV 89109.

456. Bob Newell, Kaiser Engineers - Handford, P.0. Box 888, Richland, WA 99352

457. F. Niedorkoflor, Jr., Dir, Inventory and Supply Fund Management Service (928), Veterans Adninistration, 810 Vermont Avenus, N.W., Hashington, DC 20240

458. P. A. Nikbakht, Environmental Protection Agency, 1505 Michaux Rd., Chapel HIII, NC 27514

459. NUS Corporation Library, 900 Trail Ridge Road, Aiken, SC 29801

460. John A. Huzback, GE/KAPL, Bldg. 2-5, P.0. Box 1072, Schenectady, NY 12301

461. Van Nguyen, DOE, DP-12, Germantown, Washington, DC 20545

462. Richard F. Orthen, wUS Corporation-Savannah Rivel Center, 900 Trail Ridge Road, Aiken, SC 29803

463. Cynthia Ortiz, U.S. Department of Energy, NV Technical Library, P.0. Box 98518, M.S. 505, Las Vegas, NV 89193-8518

464. Beth Osheime, U.S. Department of Energy, P.0. Box 398705, Cincinnati, OH 45239-8705 
465. Dr. W.s. Deburn, Jr., Enviromental Protection Speciallist, Div. P \& T, U.S. DOE, Washington, DC 20545

466. Colleen Ostrowskl, EH-23, U.S. Department of Energy, 1000 Independence Avenue, Washington, DC 20585

467. Kirk W. Owens, EM+331, DOE Headquarters, Washingron, DC 20545

468. Lul. Palmer, Defense Logistics Agency, Defense Reutill zation and Markecing Service, 7/6 North Washington St., Batt!. Creek, MI 49017

469. Mr. Thomas Palmierl, Office of Mngt and Budget, New Exec. Office Bldg., 8002, 726 Jackson Place, W.W., Washington, DC 20503

470. Dr. Robert H. Paret, $123 \mathrm{H}$. Tennessee Avenwe, suite 408, Oak Ridge, TN 37830

471. Jerry Parker, RH-333, Rm 7F-070, DOE, Washington, DC 20546

472. Michael Parker, GE Neutron Devices Department, P.0. Box 2908, M/S 040, Largo, FL 34643

473. Joe Parrette, WINCO, P.O. BOx 4000, Idaho Falls, ID 83403-4000

474. Ron Pauer, Lawrence Berkeley National Laboratory, No.1 Cyclotron Road, Mail Stop B75B, Berkeley, CA 94720

475. Marcus Paucock, 101515 th St., NH, Sulte 500, Washington, DC 20005

476. Ray Polletier, EH-23, GA-076, U.S. Department of Energy, 1000 Independence Ave., Washington, DC 20585

477. Donna Perla, EPA, OS-320W, 401 M street SW, Hashington, DC 20460

478. Patrick Pesacreta, EPA OS-323, 401 M Street, S.W, Waghington, DC 20465

479. Hal Peterson, U.S. Department of Energy, EH-23\%, 1000 independence Avenue, Washington, DC 20585

480. Mark Petts, EH-239, GA-076, U.S. zartment of Energy, 1000 Independence Ave., Washington, DC 20585

481. J. A. Phoenix, U.S. Department of Energy, Los Alamos Area Office, 52835 th Street, Los Alanos, NM 87544

482. L. J. Piccin, General Electric Co., P.0. Box 1072, Kessolring Site Bldg. 18, Room 213, Schenectady, NY 12301

483. Kathy Pierce, BPA, RPPD, P.0. BOX 3621, Portland, OR 97208

484. Jo Anne Pigg, Sandia National Laboratory, Div. 3311, P.0. Box 5800, Albuquerque, NM 87185

485. John Podgursky, Hest inghouse-NRF, P.0. Box 2068, Idaho Falls, 1083401

486. Jane Powers, EH-231, GA-076, DOE-HQ, Forrestal Building, 1000 Indeperidence Avenue, Washington, DC 20585

487. Keith R. Price, Battelle Northwest, MS-K613, P.0. Box 999 , Richland, Wh 99352

488. Public Health Service - Environmental Officer, Parklawn 17A-10, 5600 fishers Lane, Rockville, Mo 20857

489. Tom Pysto, SAIC, Mail Stop 517/T19, 101 Convention Center Drive, Sulte 407, Las Vegas, NV 89109

490. Richard Raior.e, ECOTEK, P.0. Box 369 , Erwin, TN 37650

491. James E. Randall, DOE/AL/OCC, P.0. Box 5400, Albuquerque, NM 871115

492. Richard Rapoport, Pacific Northwest Laboratory, 370 L'Enfarit Promenade, Suite 900, Washington, DC 20024-2115 
493. TT20 Records Center Coordinator, DIvision 3221, Sandia Nat Ional Laborator fes, P.0. Box 5800, Albuquerqua, NH 87185

494. Julle A. Reddlck, J. A. Reddlok Techinical Services, Rte 1, Box 5670, W. Richland, WA 99352

495. Randy F. Reddick, CDM Foderal Progtums Corporation, 6400 Uptown Blvd. NE, Albuquerque, NM 87110

496. Dr. Thomas E. Reed, Bettis Atomic Power Laboratory, P.0. Box 79, West Mifflin, PA $15122 \cdot 0079$

497. Chelly Reesman, Argurne Area Office - West, P.0. Box 2528, Idaho Falla, 10 83403-2528

498. Ed Regnier, EH-231, GA-076, U.S. Departrrent of Energy, 1000 Independence Avenue, Washington, DC 20585

499. Jay Rhoderick, EM-342, U.S. DOE, Trevion 11, 12800 Middl ebrook Rd., Germantown, MD 20874

500. HIllian U. Rhodes III, Arthur D. Little, Inc., Acorn Park, Cambridge, MA 02140 2300

501. Thomas Risse, DLA-W, Installation Services \& Enviromental Protection, Cameron Station, Alexandria, VA $22314-6100$

502. David Rizor, EQ80 Idaho, P.O. Box 1625, MS-8102, Idaho Falls, ID 83415-8102

503. Cary Roles, EH-232, U.S. Department of Energy, 1000 Independence Avenue, Washington, DC 20585

504. D. M. ROss, EH-34, 0-154, U.S. Department of Energy, Washington, DC 2.0545

505. Wayne Ross, Battelle-Northwest, P.0. Box 999, MS. P7-41, Richland, WR, 99352

506. Tom Rowland, Hest Valley Project Office, P.0. Box 191, Hest Valley, NY 14171-0191

507. Mell J. Roy, U.S. DOE, Rocky Flats Office, P.0. Box 928, Golden, Co 80402-0928

508. William Roy-Herrison, U.S. DOE, DP-12, GTN, Washington, DC 20545

509. Julie Russlan, Advanced sciences, Inc., 2000 N. 15th st., suite 407, Arlington, VA 22201

510. D. J. RYan, KAPL, P.O. Box 1072, Mail stap 01-289, schenectady, NY 12301

511. Marie Saint Louis, EM-55, 12800 Middlebrook R.d., Germantown, MO 20874

512. R. D. Sanders, Sr., Hanager of Envirommental Compl iance and Regulatory Affairs, Chem-Nuclear Geotek, P.O. Box 1400, Grand Junction, CO 81502-5504

513. Sandia National Laboratories, Envirormental Prograns, P.0. Box 5800, Albuquerque, NM 87185

514. M. D. Sandvig, Manager of Heal th, Safety and Security Planning and Oversight, Chem-Nuclear Geotok, P.0. Box 1400, Grand Junction, CO 81502-5504

515. Willien Savage, ME-13, E-461, U.S. Department of Energy, Washington, DC 20545

516. Rancty Sayer, EGrG Idaho, P.0. Box 1645, MS 9206, Idaho Falls, ID 83415

517. Lt. Col. Bernie Schafor, HQ ESAC/JACE, Bolling AFB, DC 20332

518. Keith schardein, IT Corporation, 5301 Central Avenue ME, suite 700, Albuquerque, NM 87108

519. John Scheer, Defense Logistics Agency, DLA-WH, Comeron Station, Alexandria, VA 22304-6100

520. C. F. Schimikowski, General Electric Co., P.0. Box 1072, Bldg. A1, Rm. 227, schenectrady, NY 12301

521. John J. Schinkle, Director, Safety Programs Division, U. S. Department of Energy, Albuquerque Operations Office, P. O. Box 5400, Albuquerque, NM 87115

522. Robert L. Schlegel, NUS Corporation, 910 Clopper Road, P.0. Box 6032, Gaithersburg, MD $20877-0962$ 
523. Kathleen Schmidt, U.S. DOE, EH-231, 1000 Independence Avenue, Washington, DC 20585

524. Owen C. Schmidt-APp, BPA, P.O. Box 3621, Portland, OR 97208

525. Wendy Schmier, Westinghouse Idaho Nuclear Co., Inc., 1955 Freemont Avenue, P.0. Box 4000, Idaho Falls, id $83403-5111$

526. M. Paul Schmierbach, Chief, Tennessee Valley Authority, 226 summer Place Building, 300 Sumit Hill Drive, Knoxville, TN 37902

527. Craig Schmit, Energy \& Environmental Research Center, University of North Dakota, Box 8213, University Station, Grand Forks, ND 58202

528. Sue Schneider, WEMCO, P.0. Box 398704, Cincinnati, OH 45239-8704

529. Allen Schubert, RCRA Progran, T-441A, Rockwell International, Rocky Flats Plant, P.O. Box 464, Golden, co 80402-0464

530. Science Applications International Corporation, 405 S. 8th St., Suite 201, Boise, 1083702

531. Randy Scioct, EH-24, 3E-094, U.S. Department of Energy, 1000 Independence Avenue, Hashington, DC 20585

532. Secretary of Air Force, SAS/MIQ, Rm 4C-916, Pentagon, DC 20330

533. A. R. Seepo, Schenectady Navsl Reactors Office, P.0. Box 1069, Schenectady, NY 12301

534. Kevin Selby, Battelle-PNL, P. O. Box 999, MISN: P7-68, Richland, WA 99352

535. Richard F. Senk, U.S. DOE/EHD, P. O. BOX 5400, Albuquerque, AM 87115

536. John Shaffer, Enviromental Division, U.S. Department of Energy, P. O. Box A, Aiken, SC 29802

537. Dr. Richard B. Sheldon, General Electric Company, P.D. Box 8, Bldg. K-1, Room 3A15, Schenectady, NY 12301

538. Major Douglas R. Sherwood, 8535 Cirrus Court, Colorado Springs, CO 80920

539. George Sherwood, WE-82, E-451A, U.S. Department of Energy, Weshington, DC 20545

540. Jean Shorett, PNL, 370 L'Enfant Promenade, Suite 900, Washington, DC 20024-2115

541. J. Lymn sichelstiel, $\mathrm{CH}_{2} M H i l l, 599$ Oak Ridge Turnpike, Oak Ridge, TW 37830

542. Ar. Ton Sims, Director, Environmental Plarning Division, Air force Regional Civil EngineerEastern Region, AFRCE-ER/ROV, 77 Forsyth St. S.W., Atlanta, GA 30335-6801

543. J. E. Sinor, 7960 Nimot Road, Box 649, Hiwot, CO 80544

544. Peter Smiel, Knolls Atomic Power Lab, Kesselring site Operations, 30050 Atomic Project Road, Ballston Spa, NY 12020-2817

545. D. Smith, DOE-HQ, Forrestal Bldg, DP-50, 1000 Independence Ave., Washington, DC 20585

546. Melissa H. Smith, Director, ES\&H Division, DOE, Strategic Petroleum Reserve Project Management Office, 900 Cormerce Road East, New Orleans, LA 70123

547. Ronald J. Smith, Analytical Resources, Inc., B79 fritztown Road, P.0. Box 2194, Reading, PA 19608

548. Gary Snodgrass, Hevada Operations Office, P.0. Box 98518, Las Vegas, NV 89109

549. C.A. Snyder, Bldg. T130B, EG\&G Rocky Flats Inc., P.0. Box 464, Golden, CO B0402

550. D. J. Sommer, Westinghouse, P. O. Box 1970, Richland, WR 99352

551. Dale Sorensen, Babcock \& Wilcox, P.0. Box 1469, MS-0301, Idaho Falls, ID 83403

552. David Stair, Bechtel, P.O. Box 350, Oak Ridge, TN 37831-0350 
553. Chris Staley, EG\&G Idaho, P.O. Box 1625, Idaho Falls, ID 83415-2110

554. Dr. J. B. States, Mgr., Idaho Falls Operations Office, Advanced Sciences, Inc., 477 North Shoup Avenue, Idaho Falls, ID $83402-3658$

555. Franklin Steans, Department of the Interior, Office of Env Project Review, 18th \& C Streets, W.W., Washington, DC 20240

556. Lee stevens, U.S. DOE, EM-331, Trevion II Bldg., GTH, Washington, DC 20585

557. Jay Stimmel, Los Alamos National Laboratory, Mail Stop C920, Los Alamos, NM 87544

558. Larry stirling, EH-231, 3G-089, U.S. Depertment of Energy, 1000 Independence Ave., Washington, DC 20585

559. Maryenne Strain, Knolls Atomic Power Laboratory, P.0. Box 545, Hindsor, CY 06095

560. Maureen Sullivan, Department. of Defense Enviromental Office, 400 Army Navy Drive, \#206, Arlington, VA 22202-2884

561. Echo Summers, SAIC, 101 S. Parke Ave., Suite 5, P.0. Box 50697, Idaho Falls, ID 83405

562. John Surmeir, Nuclear Regulatory Comm, Division of Waste Mngt, Mail Stop ss623, Washington, DC 20555

563. Denise Swink, FE-2, 4G-064, U.S. Department of Energy, 1000 Independence Avenue, Washington, DC 20585

564. Anthony SY, DOE, San Francisco Operations Office/ER, 1333 BroBdway, Oakland, CA 94612

565. Anthony L. Takacs, Department of Energy, P.0. Box 700, Piketon, OH 45661

566. George Talley, Los Alamos National Laboratory, P.O. Box TA59, K-499, Los Alamos, NM 87544 (disk copy also)

567. Darmin N. Taras, US Army Material Command, AMCSF, 5001 Eisenhower Avenue, Alexandria, VA 22333

568. Bruce W. Taylor, General Electric-Knolls Atomic Power Laboratory, P.0. Box 545, Windsar, CT 06095

569. Dr. David W. Taylor, SAIC, P.0. Box 2501, 800 Oak Ridge Turnpike, Oak Ridge, IN 37831

570. Thomas Taylor, Professional Analysis, Inc., 1050 E. Flamingo Road, Suite 367, Las Vegas, NV 89119

571. Michael Teresinski, Basic Energy Sciences, U.S. DOE, Room ER-13, 1000 Independence Ave., SW, Washington, DC 20585

572. Stephen G. Termaath, COL,USAF (ret), Engineering-Science, Inc., 10521 Rosehaven Street, Fairfax, VA 22030-2899

573. Charles Terrell, Ecological Sciences Division, Soil Conservation Service, P. O. Box 2890 , Washington, DC 20013

574. Carolyn Thompson, EH-231, U.S. Department of Energy, 1000 Independence Ave., Hashington, DC 20585

575-576. Lois Thompson, EH-231, GA-076, U.S. Department of Energy, 1000 Independence Ave., Hashington, DC 20585

577. Robert Thompson, A-104, Enviromental Protection Agency, Office of Federal Activities, 401 M Street, S.W., Mashington, DC 20460

578. Donne Thurston, c/0 SAIC MS 2-5-1, 1710 Goodridge Drive, McLean, VA 22102

579. David Tolle, U.S. DOE, Bldg. 617, Argonne National Laboratory, 9700 S. Cass Avenue, Argonne, IL 60439

580. Bob Toro, BDM, Inc., 12850 middlebrook Road, suite 300, Germantown, MD 20874 
581. Ken Torrisi, AME-463, FM NeH England Region, 12 New England Executive Park, Burlington, MA 01803

582. Ton Traceski, EH-232, GA-076, U.S. Depertment of Energy, 1000 Independence Avenue, Heshington, DC 20585

583. Thomas M. Treger, U.S. DOE, Envirommental Restoration Division, Savannah River Operations Office, P.O. Box A, Aiken, SC 29802

584. Jerry Truitt, SAIC, P.O. Box 2501, 800 Oak Ridge Turnpike, Oak Ridge, TN 37831-2501

585. Kelly Tzoumis, DOE NTR EIS Review Group, 901 D street, NW, suite 90C, Washington, DC 20024

586. R. G. Upchurch, Westinghouse Hanford Company, P.O. Box 1970, Mail Stop H4-50, Richland, WA 99352

587. U.S. AEHA, Air Pollution Engineering Division, Attn: HSHB-ME-A/Major Pllcher, Aberdeen Proving Ground, MD 21010-5422

588. U.S. Department of Energy, P.O. Box 5400, Albuquerque Operations Office, Management Support Division, Albuquerque, AM 87115

589. U.S. Department of Energy, Albuquerque Operations office, Environmental Protection Division, P.0. Box 5400, Albuquerque, NM 87115

5120. U.S. Department of Energy, Savannah River Ops. Office, Environmental Division, P.O. Box A, Aiken, SC 29801

591. Edward Vallario, EH-33, G-122, U.S. Department of Energy, Washington, DC 20545

592. Tim Van EPP, $\mathrm{CH}_{2} \mathrm{MH}$ Hill, P.O. Box 4400, Reston, VA 22090

593. Labell Vance, W4-16, Hestinghouse Hanford, P.O. Box 1970, Richland, WA 99352

594. Rebecca Vanscyoc, 9474 Kearny Villa Rd., suite 103, San Diego, CA 92126

595. Arthur T. Vollmer, Jacobs Engineering Group, Inc., 5301 Central Avense, NE, Suite 1400, Albuquerque, MAM 87108

596. Thomas Hajtalik, Signal Place 4 Morth 32 C, 1100 Market Street, Chattanooga, TN 37402

597. Rob Waldman, Alaska Power Administration, Environmental, Safety, and Health Manager, P.0. Box 020050, Juneau, AK 99802

598. Dennis Halker, EG\&G Idaho Inc., 1955 fremont Avenue, P.O. Box 1625, MS-3413, Idaho Falls, ID $83415-3414$

599. Juliet Walker, SAIC, 1710 Goodridge Drive, Mail Stop T2-5-1, McClean, VA 22101

600. Andy Hallo, EH-231, Gh-076, U.S. Department of Energy, 1000 Independence Ave., Washington, DC 20585

601. Edward Wandelt, USPS, Real Estate and Bldg. Dept., $4 \pi 5$ L'Enfant Plaza West, SW, Washington, DC 20260-6400

602. W. E. Ward, General Electric Co., P.0. Box 545, Bldg. 1, Windsor, CT 06095

603. Regan S. Weeks, Westinghouse Manford Company, P.O. Box 1970, Mailstop H4-57, Richland, WA 99352

604. Randy Weigel, Westinghouse Savannah River Company, Environmental Protection Department, Bldg. 742-A, P.0. Box 616, Aiken, SC 29802

605. Roy F. Weston, Inc., 12800 Middlebrook Road, \#207, Germantown, MD 20874-5201

606. Bill thite, U.S. Department of Energy, Chicago Operations Office, 9800 South Cass Avenue, Argorne, IL 60439

607. Beverly Whitehead, U.S. DOE, EH-231, 1000 Independence Avenue, Washington, DC 20585

608. A. E. Whiteman, U.S. DOE, Rocky flats Area Office, P.0. Box 928, Golden, 6080401 
609. William M. Wierzbicki, Building 707-F, Savannah River Site, Aiken, SC 29808

610. Debra J. Wilcox, Attorney, Ëcology Environment, Inc.. 950 Energy Drive, Idaho Falls, ID 83402

611. Ted Willians, EH-22, 4G-036, U.S. DOE, 1000 Independence Ave., Washington, DC 20585

612. Dr. W. Alexander Hillians, A-104, Enviromental Protection Agency, Office of Federal Activities, 401 M Street, S.H., Rm. 2125, Hashington, DC 20460

613. Scott Willis, U.S. DOE, Alaska Power Administration, P.0. Box 50, Juneau, AK 99802

614. Michele Hilson, Westinghouse Savannah River Company, Environmental Protect Department, Building 742A, P.0. Box 616, Aiken, SC 29802

615. Eileen M. Wisser, WSO-6, P.O. Box 545, Windsor, CT 06095

616. Frank J. Wobber, Division of Ecological Research, Office of Health and Environmental Research, Office of Energy Research, MS-E201, Department of Energy, Washington, DC 20545

617. Mr. Paul J. Yaroschak, Dep. Dir., Enviromental Quality Div, Maval Fac. Engr. Command (Code-112), 200 Stovali Street, Alexandria, VA 22332

618. Veronica Ybarra, Jacobs Engineering Group, 5301 Central Avenue, NE, suite 1600, Albuquerque, NM 87108

619. Katherine L. Yuracko, Office of Management and Budget, 725 17th Street N.H., Room 8002 , Washington, OC 20503

620-629. Office of Scientific and Technical Information, P.0. B0X 62, Onk Ridge, TN 37831

630. OR-DOE, Assistant Manager, Energy Research and Development 

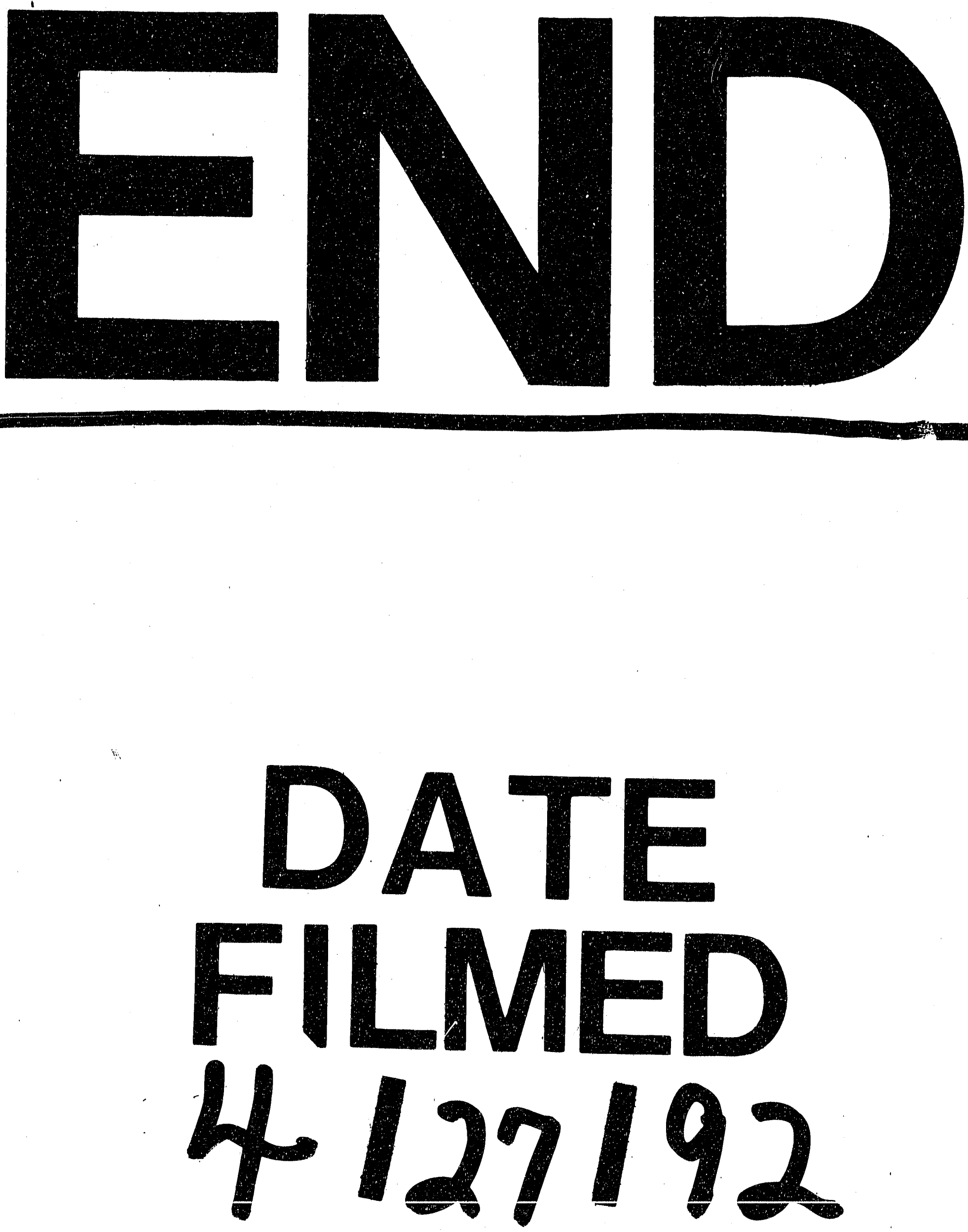


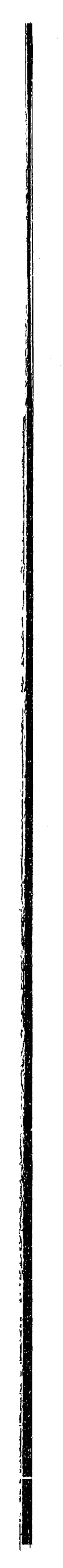

